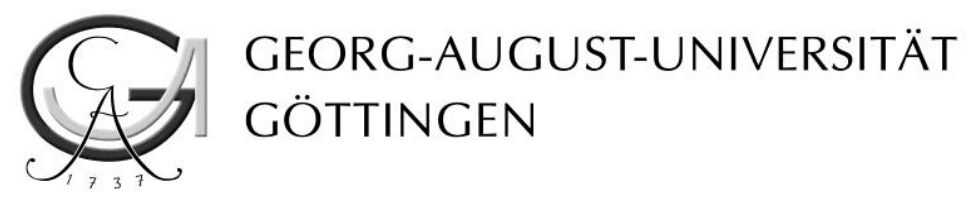

\title{
„Characterisation of the immune modulatory effect of wild type Rift Valley fever virus strains"6
}

\author{
Dissertation
}

zur Erlangung des mathematisch-naturwissenschaftlichen Doktorgrades

„Doctor rerum naturalium“

der Georg-August-Universität Göttingen

\author{
vorgelegt von \\ Modou Moustapha Lo \\ aus Gossas (Senegal)
}

Göttingen 2010 
Referent:

Prof. Dr. med. Gerhard Hunsmann

Koreferent:

Prof. Dr. med. Uwe Groß

Tag der mündlichen Prüfung:

26. Oktober 2010 


\section{Contents}

Abbreviations

1 Introduction.....................................................................................................................5

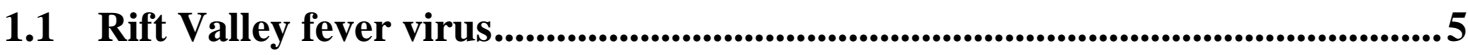

1.1.1 RVFV infections, symptoms and pathogenicity .............................................8

1.1.2 RVFV epidemiology ...........................................................................

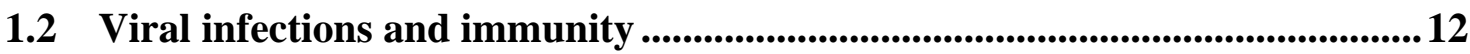

1.2.1 Interference of RVFV with the IFN system................................................14

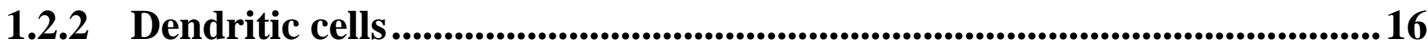

1.2.2.1 Plasmacytoid dendritic cells...................................................................16

1.2.2.2 Conventional dendritic cells...........................................................18

1.2.2.3 Cytokine induced modulation of immune responses mediated by DCs ..........................................................................................................20

1.3 Aim of the study ..................................................................................................................20

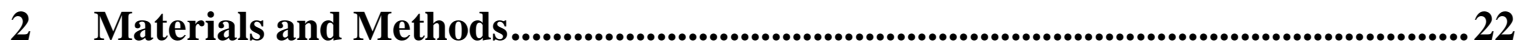

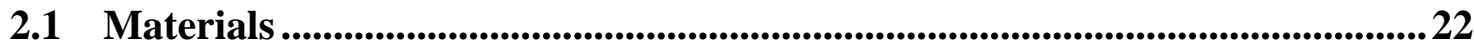

2.1.1 Bacterial strains ................................................................................................ 22

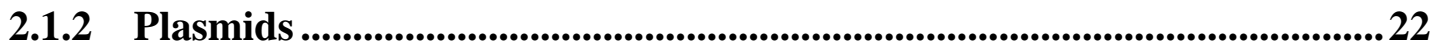

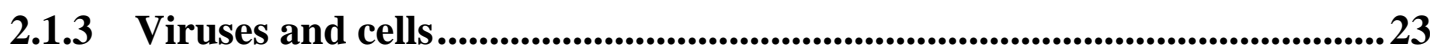

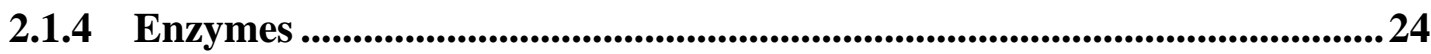

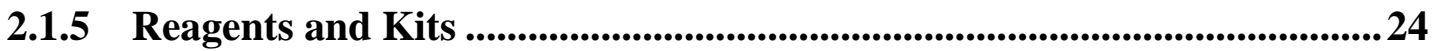

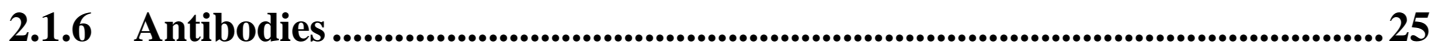

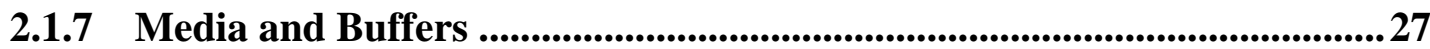

2.1.8 Chemicals.......................................................................................................228

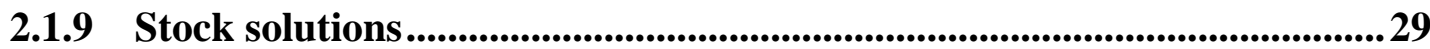

2.1.10 Laboratory equipment .........................................................................30

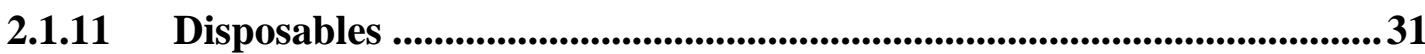

2.1.12 Computer software ..................................................................................32

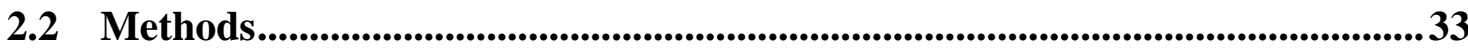

2.2.1 Manipulation of nucleic acid...............................................................33

2.2.1.1 cDNA preparation.............................................................................33

2.2.1.2 Amplification of cDNA and preparation of PCR product for ligation 33

2.2.1.3 Agarose gel electrophoresis of PCR products .......................................34

2.2.2 Cloning experiments ..............................................................................34

2.2.2.1 Dephosphorylation of plasmids ................................................................34

2.2.2.2 Ligation of PCR products into pCRII vector ......................................34

2.2.2.3 Transformation of pCRII vector ........................................................34 
2.2.2.4 Ligation of PCR products into pI.18 ....................................................35

2.2.2.5 Transformation of pI.18 .............................................................................35

2.2.2.6 Plasmid extraction ............................................................................35

2.2.2.7 Restriction of amplificates and recombinant plasmids and

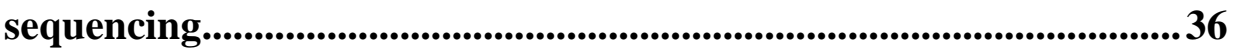

2.2.3 Expression and detection of RVFV-NSs .................................................36

2.2.3.1 Assessment of the IFN- $\beta$ promoter activation by RVFV-NSs proteins 36

2.2.3.2 Monitoring NSs filament formation in the nucleus of infected Vero E6 cells by indirect immunofluorescence assay ...................................3.37

2.2.4 Manipulation of cells ..........................................................................38

2.2.4.1 Preparation and infection of plasmacytoid dendritic cells..................38

2.2.4.2 Preparation and infection of myeloid dendritic cells..........................38

2.2.4.3 Vero E6 culture and virus stock generation .......................................39

2.2.4.4 RVFV infections of Vero E6 cells in the presence of IL-6..................39

2.2.4.5 RVFV infection of DCs...............................................................40

2.2.5 Flow cytometry analysis .........................................................................................40

2.2.6 Cytokine detection .....................................................................................................41

2.2.6.1 Monitoring proinflammatory cytokine production in DC supernatants after RVFV infection ..................................................41

2.2.6.2 Monitoring interferon- $\alpha$ production in DC supernatants after RVFV infection........................................................................................41

2.2.7 Tissue Culture Infectious Dose 50 (TCID50) assays................................41

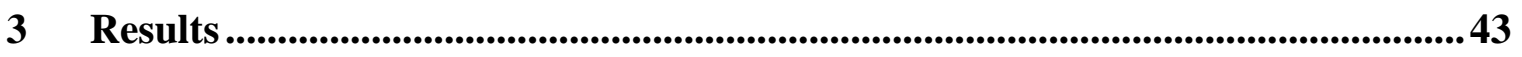

3.1 Cloning of NSs of RVFV isolates into the eukaryotic expression vector pI.18 .

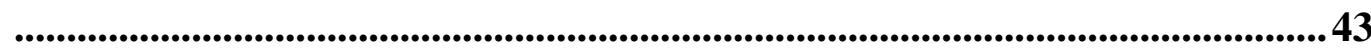

3.2 Inhibition of IFN- $\beta$ promoter activation by RVFV-NSs ................................44

3.2.1 Comparison of the IFN- $\beta$ promoter suppressing activity of the RVFV-

NSs clones with the reference wild-type ZH548 RVFV-NSs..............................45

3.3 Comparison of filament formation in the nucleus of Vero cells transfected with 26 RVFV-NSs expression plasmids

3.4 A point mutation in the NSs sequence of RVFV-NSs R7 affects NSs function .

3.5 A point mutation in the NSs sequence of RVFV-NSs R10 affects filament

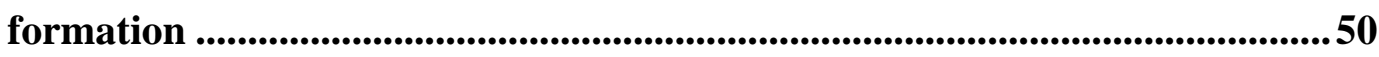

3.6 RVFV replicates in mDCs but does not in pDCs ..........................................50

3.6.1 Proinflammatory cytokine production in mDCs infected by RVFV.......53

3.6.2 Proinflammatory cytokine production in pDCs infected by RVFV ........55

3.7 Effect of IL-6 on RVFV replication............................................................57 
3.8 Interferon- $\alpha$ production of DCs infected with RVFV .58

3.9 Comparison of IL-6 and IFN- $\alpha$ production of pDCs infected with RVFV and TBEV. . .60

3.10 Expression and regulation of surface marker molecules in mDCs infected by RVFV

3.11 Expression and regulation of surface marker molecules in pDCs infected by RVFV

4 Discussion .71

4.1 Natural variability of NSs function ................................................................

4.1.1 Rift Valley Fever NSs variants suppressing IFN- $\beta$...................................71

4.1.2 Rift Valley Fever NSs variants not suppressing IFN- $\beta$............................ 72

4.2 Characterisation of the interaction of RVFV with antigen presenting cells .74

4.2.1 Replication of RVFV in dendritic cells and cytokine profile induced ....76

4.2.2 The interferon response of dendritic cells infected with RVFV ..............78

4.2.3 Activation patterns of dendritic cells infected with RVFV ......................79

4.2.3.1 Activation of adhesion markers..............................................................8 80

4.2.3.2 Activation of maturation marker CD83 ..............................................8.81

4.2.3.3 Activation of costimulatory markers ...................................................881

4.2.3.4 Activation of MHC markers ....................................................................81

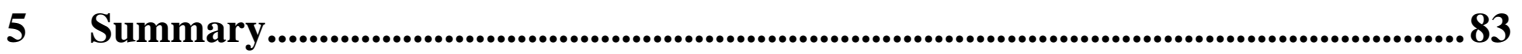

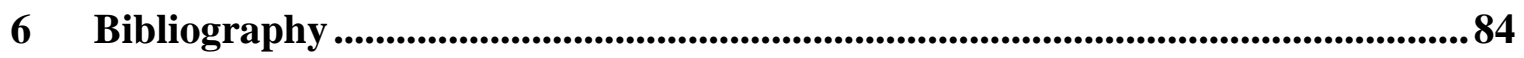

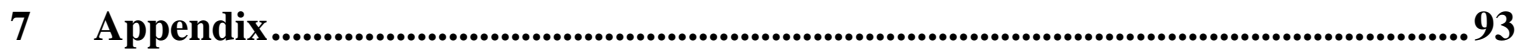

7.1 Additional results of proinflammatory cytokine production in mDCs infected by RVFV (chapter 3.6.1) ........................................................................................993

7.2 Additional results of proinflammatory cytokine production in $\mathrm{mDCs}$ infected by RVFV (chapter 3.6.1) .................................................................................95

7.3 Additional results of proinflammatory cytokine production in pDCs infected by RVFV (chapter 3.6.2) .................................................................................................97

7.4 Additional results for expression and regulation of surface marker molecules in mDCs infected by RVFV (Chapter 3.10) ..................................................100

7.5 Additionnal results of expression and regulation of surface marker molecules in pDCs infected by RVFV (chapter 3.11) ...........................................................104

7.6 Complete alignment of NSs sequences of the 26 RVFV strains analyzed in this thesis (Chapters 3.4 and 3.5). 


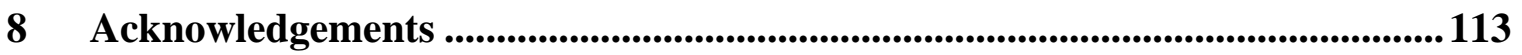

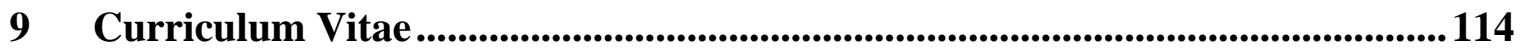




\section{Abbreviations}

Table 1. Abbreviations

$\begin{array}{ll}\text { AA } & \text { Amino acid } \\ \text { Ab. } & \text { Antibody } \\ \text { Amp } & \text { Ampicilline } \\ \text { ATF2 } & \text { Activating transcription factor 2 } \\ \text { ATP } & \text { Adenosine-Triphosphate } \\ \text { BDCA2 } & \text { Blood dendritic cell antigen 2 } \\ \text { Bp } & \text { Basepair } \\ \text { BSA } & \text { Bovine Serum Albumin } \\ \text { CCR } & \text { Chemokine chemo receptor } \\ \text { CD } & \text { Cluster of differentiation } \\ \text { cDNA } & \text { Complementary DNA } \\ \text { cm } & \text { Centimeter square } \\ \text { CO } & \\ { }^{\circ} \text { C } & \text { Carbon dioxide } \\ \text { CPE } & \text { Degree Celsius } \\ \text { ddH } 2 \mathrm{O} & \text { Cythopathogenic effect } \\ \text { Da } & \text { Double distilled water } \\ \text { DC } & \text { Dalton } \\ \text { DMP } & \text { Dendritic cell } \\ \text { DMSO } & \text { Dimethyl-pimelinediimidat-dihydrochlorid } \\ \text { DNA } & \text { Dimethyl sulfoxyde } \\ \text { dNTPs } & \text { Desoxy-ribonucleic acid } \\ \text { dsRNA } & \text { Double stranded Ribonucleic acid } \\ \text { DTT } & \text { Dithiothreitol } \\ \text { E. coli } & \text { Escherichia coli } \\ \end{array}$


EDTA Ethylene-diamine-tetra-acetic-acid

ELISA Enzyme linked immunosorbent assay

EtOH Ethanol

FACS Fluorescence Activated Cell Sorting

GM-CSF Granulocyte macrophage colony stimulating factor

h Hour

$\mathrm{H} 2 \mathrm{~A} \quad$ Histone 2A

H2B Histone $2 \mathrm{~B}$

H3 Histone 3

H4 Histone 4

HEPES N-2-Hydroxyethylpiperazin-N'-2-ethansulfoxid acid

IgG Immunglobulin $\mathrm{G}$

IFA Indirect fluorescence antibody

IFN Interferon

IgG Immunoglobulin G

IgM Immunoglobulin M

IHA Inhibition of Haemagglutination

IL Interleukin

IRF Interferon regulatory factor

IPTG Isopropyl- $\alpha$-D-Thiogalactopyranosid

$\mathrm{kb} \quad$ Kilobase

kDA Kilodalton

$\mathrm{Kg} \quad$ Kilogramme

$1 \quad$ Liter

LPS Lipopolysaccharide

Luc Luciferase

M Molar

$\mathrm{mA} \quad$ Milliampere

mg Milligram

mn Minute

ml Millilitre

mM Millimolar 
MW Molecular weight in Dalton

MHCI Major Histocompatibility complex I

MHCII Major Histocompatibility complex II

MOI Multiplicity of infection

MyD $88 \quad$ Myeloid differentiation primary response gene (88)

NF-KB Nuclear factor Kappa B

nm Nanometer

NSm Non structural m (gene)

NSs Non structural s (gene)

nt Nucleotide

PAMP Pathogen associated molecular pattern

PBMC Peripheral blood mononuclear cells

PCR Polymerase Chain Reaction

$\mathrm{pH} \quad$ Acidity

PPR Peste des petits ruminants

RNA Ribonucleic acid

RT Room temperature

RVFV Rift Valley fever virus

sec Second

SPIT Solid-phase immunosorbent technique

ss $\quad$ Single stranded

TAE Tris-Acetat-EDTA-Buffer

Taq Thermus aquaticus

TBK Tank binding kinase

TE Tris-EDTA-Buffer

TEMED N,N,N',N'-Tetramethyl-ethylen-diamine

Th T helper

TLR Toll-like receptor

TNF Tumor necrosis factor

Tris Tris-(hydroxymethyl)-amino-methane

ts Temperature sensitive

U Unit 


$\begin{array}{ll}\text { Up } & \text { Upper } \\ \text { UV } & \text { Ultraviolet } \\ \text { V } & \text { Volt } \\ \text { wtRVFV } & \text { wild type Rift Valley fever virus } \\ \text { v/v } & \text { volume/volume } \\ \text { w/v } & \text { weight/volume } \\ \beta & \text { Beta } \\ \mu & \text { Micro- }\end{array}$




\section{$1 \quad$ Introduction}

\subsection{Rift Valley fever virus}

Rift Valley fever virus (RVFV) is a member of the genus Phlebovirus in the Bunyaviridae family. Like all the members of the family it is an enveloped virus and possesses a singlestranded tripartite RNA genome composed of large (L), medium (M), and small (S) segments [1]. The segmented negative single strand RNA virus ((-) ssRNA) genome of the virus (Figure 1) codes for the polymerase (L-segment), the glycoproteins Gn and Gc and two non-structural proteins NSm14 and NSm78 (M-segment) and for the nucleocapsid (S-segment). The glycoproteins are the targets for neutralizing antibodies and influence virus cell attachment and tissue tropism. The $\mathrm{S}$ segment utilizes an ambisense strategy to code for two proteins: the nucleoprotein $\mathrm{N}$ and the nonstructural protein NSs. The two coding regions for $\mathrm{N}$ and Nss are separated by a poly $(\mathrm{C})$-rich intergenic region (in the genomic sense) of approximately 81 nucleotides (nt). The NSs of RVFV is a $31-\mathrm{kDa}$ protein, which is phosphorylated by casein kinase II at two serine residues located in the carboxy terminus. Furthermore, the carboxy-terminal domain mediates oligomerization and is responsible for filament formation in the nuclei of RVFV infected cells [2]. The completely nuclear localization of NSs is rather intriguing and surprising since RVFV, like all the members of the family Bunyaviridae, utilizes only the cytoplasm as its site for replication [3]. Intranuclear inclusions were first detected in the hepatocytes of RVFV-infected animals [4]. Later, Swanepoel and his group [5] detected nuclear filaments in cells infected with various virulent RVFV strains and showed that the nuclear filament is composed of bundles of 50-nm-thick fibrils, which occupy half the length of the nucleus and are confined exclusively to the nuclei but not associated with nucleoli. The NSs protein has been demonstrated to function in the down regulation of RNA polymerase II activity, resulting in host cell transcription shutoff and, via this mechanism, to cause antagonism of host cell interferon responses [6, 7]. 


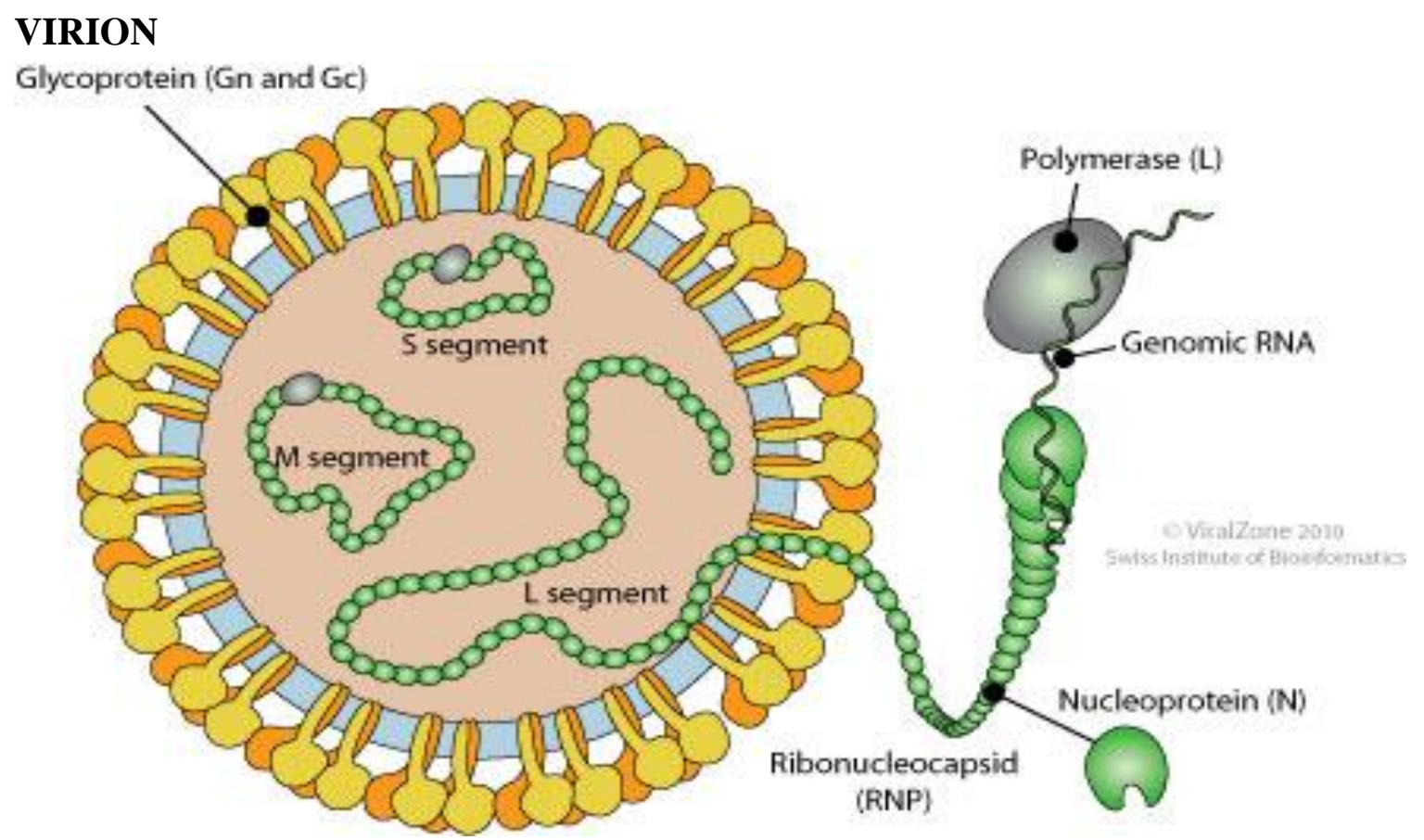

GENOME

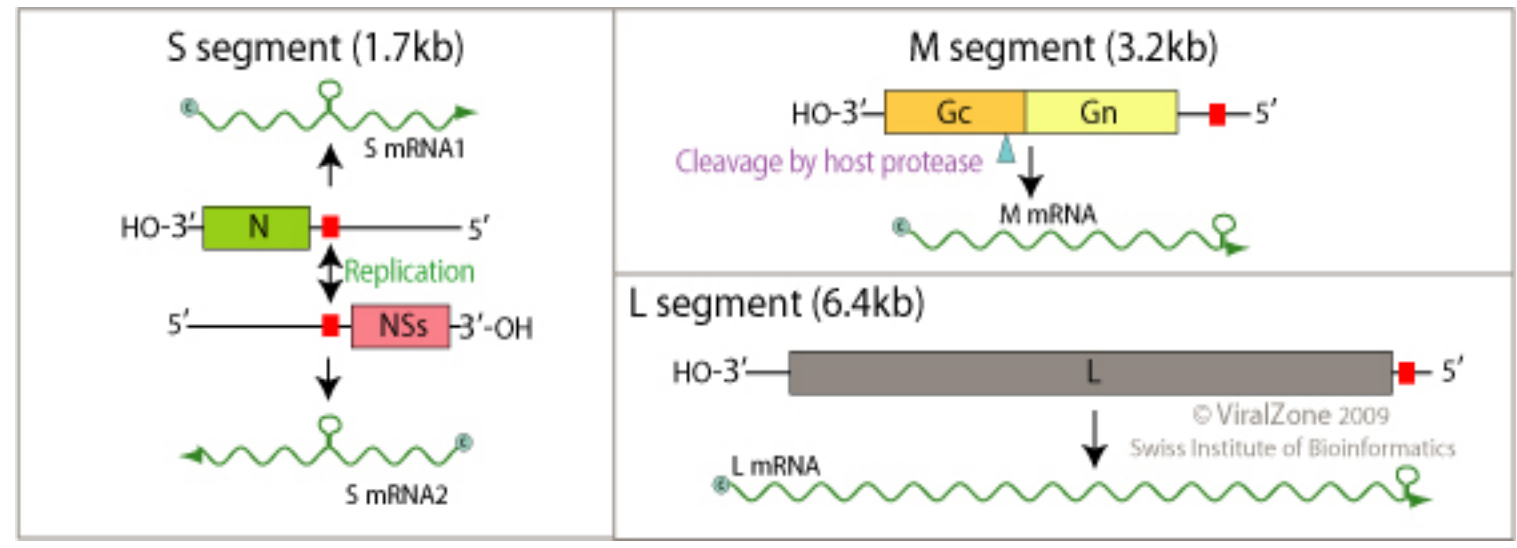

Figure 1. Schematic drawing of the structure and genome of the Phlebovirus genus, which belongs to the Bunyaviridae family [8] . Top: schematic drawing of the structure of a virus particle. Diameter: 80-120 nm. bottom: Schematic drawing of the virus genome with 3 negative oriented, single-stranded RNA-Segments: S, M and L.

The naturally attenuated RVFV strain clone 13 originally isolated from a nonfatal human case in Bangui, Central African Republic [9] carries a large in frame deletion in the NSs gene. It is not virulent in vivo (in mice and hamsters) but grows as well as wild-type RVFV in cell cultures [9].

Among natural RVFV isolates, it was found that the antigenic properties of the glycoproteins and the nucleoprotein appeared to be stable, and sequences of the Gn epitopes in the $\mathrm{M}$ segment are relatively conserved. The $\mathrm{S}$ genome sequences of five phleboviruses, Punta Toro, RVF (MP12) strain, Sandfly Fever Sicilian (SFS), Toscana and Uukuniemi viruses have already been published [10] and were shown to utilize an 
ambisense strategy to code the N protein in the genomic sense and for the NSs protein in the antigenomic sense. The $\mathrm{N}$ and NSs represent two potential distinct targets for analysis of the $\mathrm{S}$ segment. The deduced $\mathrm{N}$ protein sequences exhibit homologies ranging from 30 to $54 \%$ [11]. However, the NSs gene is much less conserved and cannot be aligned to analyze phylogenic relationship among different phleboviruses.

Since the NSs protein is the most variable protein among Phleboviruses, its use as a marker of variability was investigated [11]. The extent of conservation of the NSs gene among RVF virus isolates was unknown and this question was raised by the existence of the naturally occurring avirulent isolate clone 13 which harbours a prominent deletion in the Nss region [12].

A study of the NSs gene of various strains of RVF virus and selected from a panel of 18 RVF virus strains collected over 38 years in eight countries under epidemic and endemic conditions and isolated from a variety of host species (arthropods, cattle and human) was performed. It included the attenuated strain MP12 [13] and the neurotropic Smithburn strain (SNS) [14], which were obtained by cell passage of the parental virulent strains ZH 548 and Entebbe, respectively. The phylogenetic analysis of all strains showed two major lineages: Egyptian and Sub-Saharan with the latter lineage divided into two clusters [15].

This analysis showed that RVFV could be separated into seven distinct genetic lineages (Figure 2). RVFV strains with diverse geographic origins can be found in each lineage, which is indicative of widespread dispersal and movement of RVF virus genotypes throughout Africa [16]. 


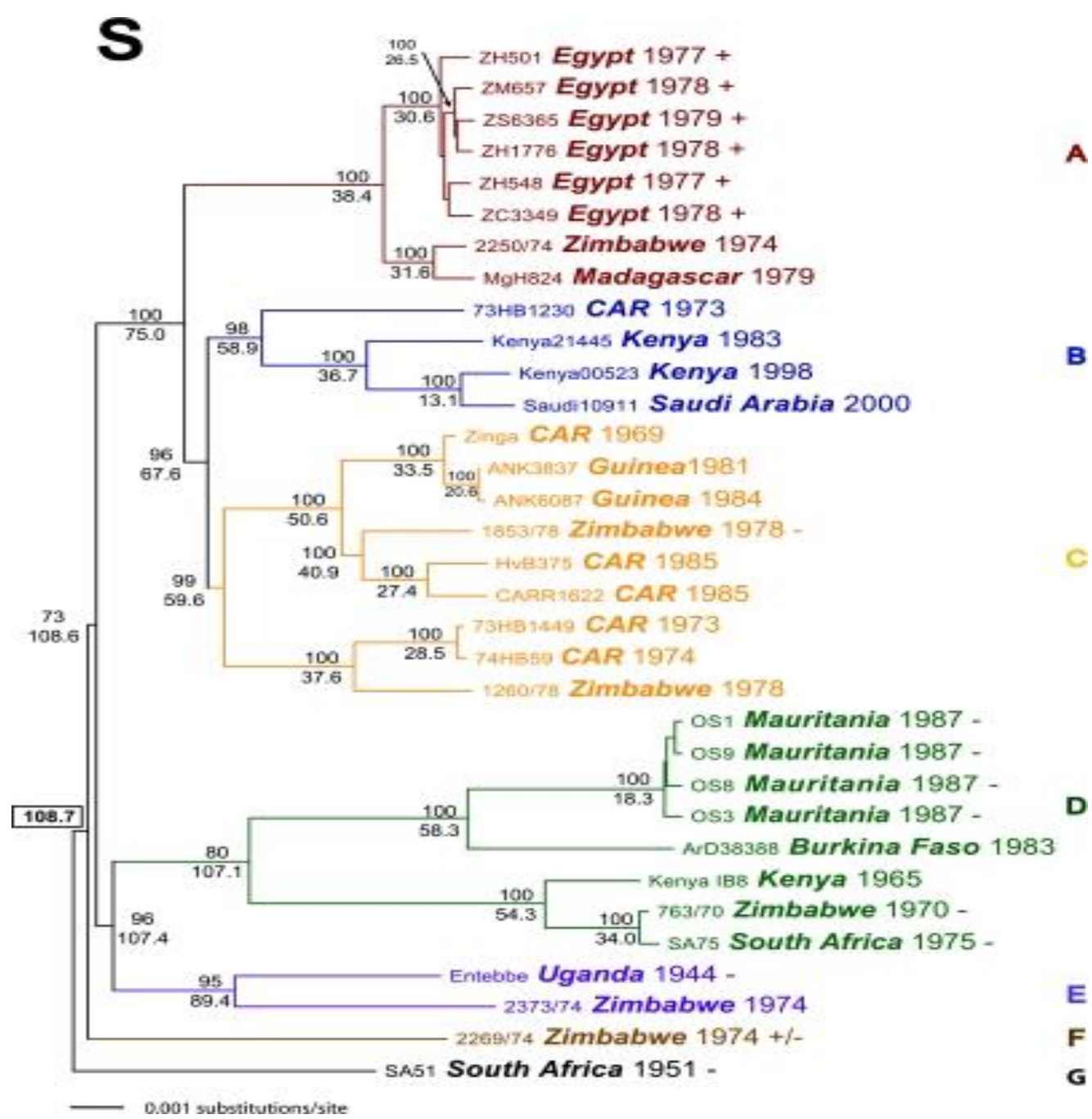

Figure 2. Diversity genetic of thirty-three $S$ segment gene of Rift Valley fever virus. Each taxon name indicates the strain, country of origin, and date of isolation. The GenBank accession numbers for the virus $S$ segments are DQ380143 to -6 , DQ380149, DQ380151 to -3 , DQ380156, and DQ380158 to -81. Also strains used in previous studies of virulence in rats are indicated with either the $(+)$ sign, which means the strain is lethal and the lethal dose $50\left(\mathrm{LD}_{50}\right)$ is $\left.\sim 1.0 \mathrm{PFU}\right)$, the $(+/-)$ signs indicates that the strain is less lethal, and the $\mathrm{LD}_{50}$ is $\left.\sim 2 \times 10^{3} \mathrm{PFU}\right)$, or the (-) sign indicates the strain is nonlethal [16].

\subsubsection{RVFV infections, symptoms and pathogenicity}

A Rift Valley fever (RVF) outbreak leading to heavy mortality in newly-born lambs on a farm in Kenya was first described in 1931 [4]. RVFV mainly causes disease in domestic ruminants inflicting a high rate of abortions and high mortality rates. Recurrent enzootic and epizootic outbreaks have been documented in eastern, southern and western Africa, Madagascar and Egypt. In 2000 it even spread to the red sea board of the Arabian Peninsula (Figure 3). The zoonotic disease can also cause epidemics in man, as recorded in Egypt in 1977, in Saudi Arabia and Yemen in 2000 and in Kenya and Tanzania in 2007 
[17-21]. RVFV survives dry periods in vertically infected eggs of different mosquito species and disease outbreaks are often linked to preceding heavy rainfalls.

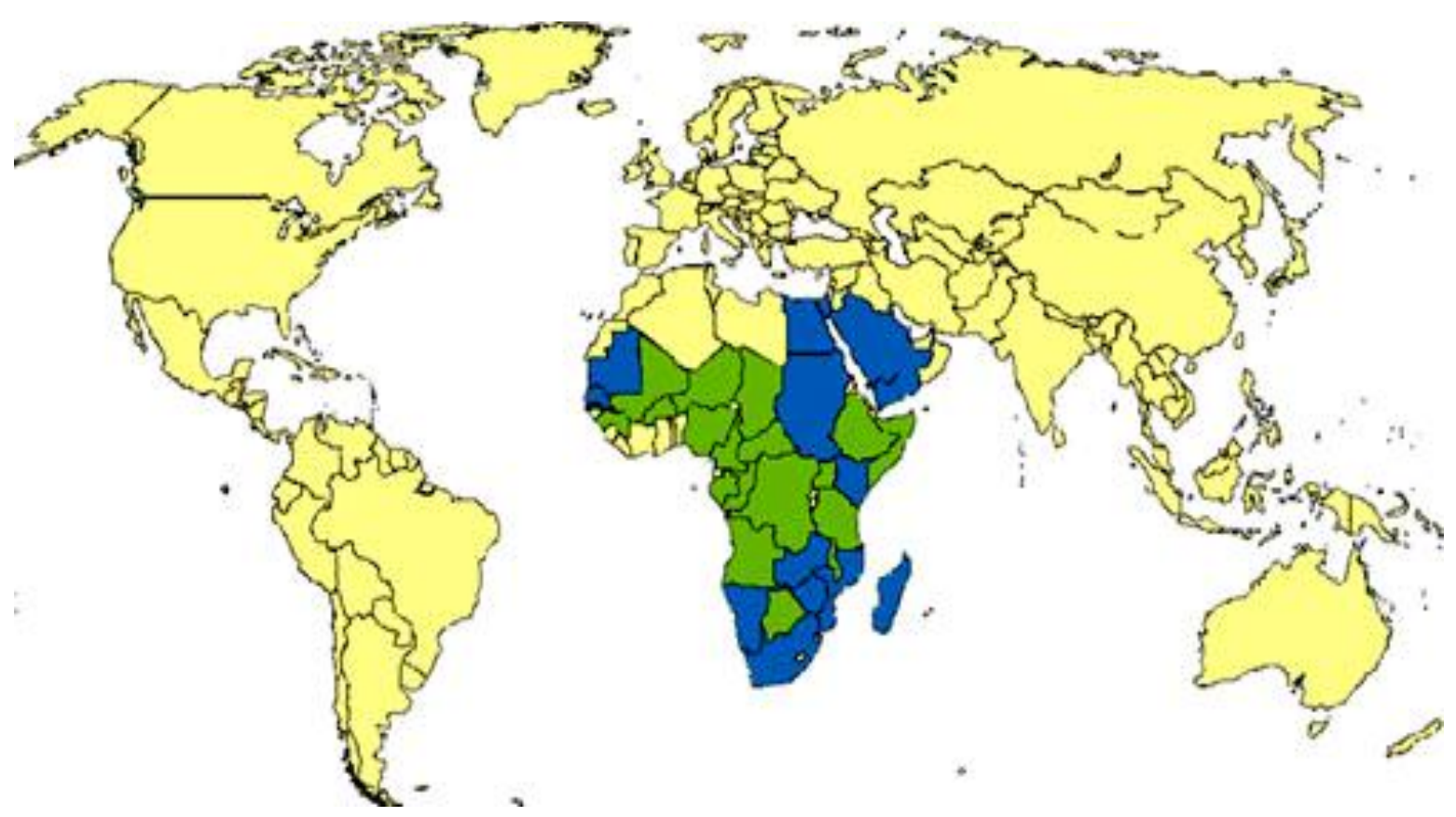

Figure 3. Distribution of Rift Valley fever in Africa. Blue, countries with endemic disease and substantial outbreaks of RVF (Gambia, Senegal, Mauritania, Namibia, South Africa, Mozambique, Zimbabwe, Zambia, Kenya, Sudan, Egypt, Madagascar, Saudi Arabia, Yemen); green, countries known to have some cases, periodic isolation of virus, or serologic evidence of RVF (Botswana, Angola, Democratic Republic of the Congo, Congo, Gabon, Cameroon, Nigeria, Central African Republic, Chad, Niger, Burkina Faso, Mali, Guinea, Tanzania, Malawi, Uganda, Ethiopia, Somalia) [22].

RVF outbreaks usually begin in livestock with elevated abortus counts. RVFV transmission to man occurs by contact to infected tissues e.g. from abortus from livestock $[23,24]$ or by aerosols from slaughtered animals [25] and consequently nomadic tribes in Kenya [26, 27] or livestock workers and wildlife rangers in Nigeria [28] show a very high seroprevalence towards RVFV. Although RVFV can spread to man by the above modes of transmission it mainly spreads by transmission via mosquitoes [29].

In humans the symptoms range from mild fever to encephalitis, retinitis and fatal hepatitis with haemorrhages. The more severe forms occur in less than $1 \%$ of patients of which up to $50 \%$ may die [30]. 


\subsubsection{RVFV epidemiology}

All known Rift Valley fever virus outbreaks in East Africa from 1950 to May 1998, and probably earlier, followed periods of abnormally high rainfall [31]. Ovine RVFV outbreaks are accompanied by abortions of sheep, goat and cattle and a high rate of mortality in lambs and calves. The biggest epidemics occur in East Africa when the El Nino/Southern Oscillation (ENSO) brings heavy increases in rainfall and large herds of European sheep and cattle breeds are present to amplify the epidemic. Because the mosquitoes that transmit Rift Valley fever lay their eggs at grass tips, eggs are submersed only during [31] periods of flooding (Figure 4), allowing for development [31]. In East Africa, early warning systems, based on Pacific and Indian Ocean (sea surface temperature) anomalies and normalized difference vegetation index (NDVI) can be used to detect animal cases at an early stage of an outbreak enabling authorities to implement measures to avert impending epidemics [31].

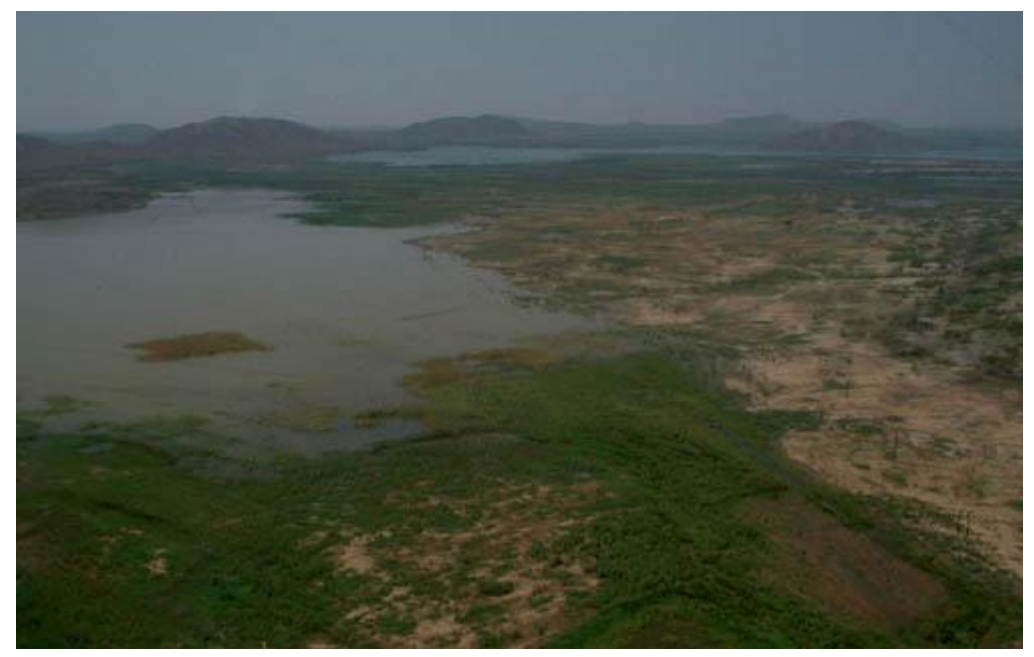

Figure 4. Photo of a typical flooded dambo area susceptible to mosquito breeding. By Roger Paskin with permission

The interepizootic vectors appeared to belong to the Neomelaniconion in East Africa, and to the subgenus Aedimorphus in West Africa [32]. RVFV was first isolated in Western Africa from Aedes (Aedimorphus) dalzieli in October 1974 in south-eastern Senegal [33]. Other isolations were reported from Aedes cumminsii and Mansonia uniformities from Burkina Faso, and Culex antennatus mosquitoes and Culicoides sp. in Nigeria, and from bats in Guinea [34]. Large RVF outbreaks in western Africa had not been reported prior to the south Mauritanian epizootic/epidemic in 1987 [35]. Serological data established an extension of the epizootic throughout Senegal and Gambia [36]. 
Flood water Aedes have drought resistant eggs, which may be able to survive several years without hatching and they require one or more floodings to trigger their development [37]. The transovarially infected mosquito eggs hatch and initiate the epidemic, which is propagated by other vectors due to the high viremias in sheep, cattle and possibly even humans. However, active RVFV transmission (Figure 5) to humans and domestic ungulates was even been recorded during a period of drought in southern Mauritania and Mali in 1982-1985 [38] indicating additional modes of transmission.

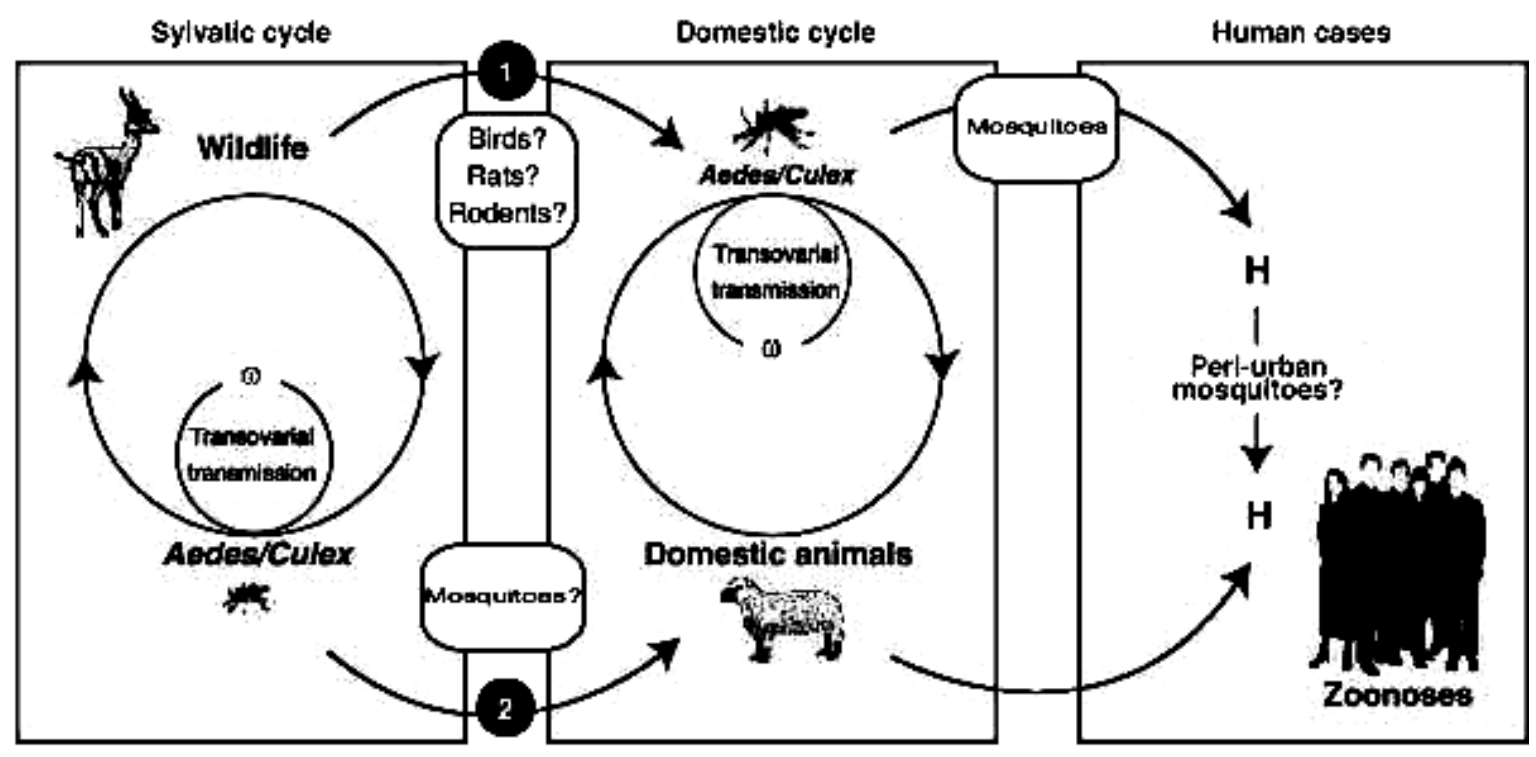

Figure 5. Theoretical cycle of Rift valley fever virus transmission by Bernard Monday from the French Institute of Research and Development [39].

As described above, many different mosquitoes and perhaps other arthropods are capable of biological transmission, including some mosquito species found in the USA [40]. A virus with this adaptability for alternative arthropod transmitters that utilizes a commonly found amplifier (e.g., sheep and cattle) must be seen as a definite risk, either through natural or bioterrorist introduction [41]. Indeed, RVFV is an emerging virus and the first RVF outbreaks outside of Africa occurred in Saudi-Arabia and Yemen in 2000. Phylogenetic comparison of the nucleotide sequence differences between the Arabian Peninsula RVFV S, M, and L segments and those of previously characterized RVFV isolates showed a close relationship between the Saudi Arabia/Yemen RVFVs and those circulating earlier in East Africa, particularly with the viruses responsible for the large RVF outbreak seen in the region in 1997-98 [42]. These results are consistent with the introduction of RVFV into Saudi Arabia and Yemen from East Africa. While genetic 
reassortment has been observed in RVFVs associated with outbreaks in various geographic regions of Africa, [43] the close phylogenetic relationship of the S, M, and L RNA segments of the 2000-01 Saudi Arabia and Yemen viruses and the earlier 1997 and 1991 Kenya and Madagascar viruses, respectively, provided no evidence of genetic reassortment among these viruses [16].

Since 1989, successive sero-surveys was conducted on selected ruminants in Senegal showed a progressive decrease of RVF antibody prevalence [44]. However, the detection of RVF immunoglobulin G (IgG) and IgM antibodies in a few young sheep and goats indicated the existence of an enzootic transmission of RVF virus in Northern Senegal [45].

\subsection{Viral infections and immunity}

Viral infections are a constant threat to higher organisms, and early detection of viruses by the innate immune system is critical for host defence. The primary host defences against virus infection are physical/chemical barriers to infection and the immune system. The immune system can be divided into two components: the innate immune system and the adaptive immune system. This division is based on the properties of the immune cell types and molecules involved in the response to infection and the speed of the response. Many advances in the field of immunity have emerged from the discovery of the Toll-like receptors (TLRs), which play a central role in driving host inflammatory responses and promoting adaptive immunity following infection and the intracellular RIG-like receptors (RLRs). TLRs and RLRs accomplish this feat by acting as host sensors of conserved structural components of viruses, bacteria, fungi, and protozoans [46, 47]. Engagement of these receptors with their respective foreign agonist drive cellular signaling, which

culminates in dynamic changes in the expression of genes that encode proinflammatory and antiviral cytokines, chemokines, and cell adhesions as well as markers of dendritic cell maturation $[48,49]$ in the case of TLRs, whereas RLRs mainly initiate an antiviral response via type I interferons (IFNs).

The response of the innate immune system to virus infection is either immediate because constitutively active or rapidly induced within hours of infection. The first response of an organism to an intruding pathogens is an inflammatory reaction that includes secretion of soluble mediators, such as cytokines and chemokines [50]. These signalling molecules 
activate or attract innate immune cells, to orchestrate an effective antimicrobial response at the site of infection. Induction of the innate immune mechanisms is not pathogen specific but is dependent on interactions between pathogenic factors and host cell determinants. A first line of host defence against viruses is mediated by the type I interferon pathway. The aim of the innate immune response is to eliminate the pathogen or to avoid spread of the infection until elimination is achieved via the adaptive immune response [51].

Double stranded RNA (dsRNA) and single-stranded RNA with a non-modified triphosphate residue at the 5' end are considered to be major pathogen associated molecules patterns (PAMPs). They are present in viral genomes and/or generated during viral transcription and replication. Then the interaction between these RNAs and retinoic acid-inducible gene I (RIG-I, 5' 3P-ssRNA) or myeloid differentiation associated gene 5 (MDA5, dsRNA) will mediate signalling via a caspase recruitment domain (CARD) to the adaptor interferon-beta promoter stimulator 1 (IPS-1) which is located in the outer mitochondrial membrane. IPS-1 in turn activates TANK-binding kinase 1 (TBK-1) which phosphorylates the constitutively expressed interferon regulated factor 3 (IRF-3) that is located in the cytoplasm. Hyperphosphorylated IRF-3 monomers dimerize and translocate in the nucleus. After interaction with the cAMP-response element-binding protein (CREB binding protein)/p300 (CBP/p300), binding of this complex to the IFN- $\beta$ promoter triggers IFN- $\beta$ transcription (Figure 6). Another possibility is the interaction of viral RNAs with the endosomal toll like receptor 3 (TLR3), TLR7, TLR8. TLR3 detects and responds to dsRNA. Through the extracellular region and a Toll/IL-I receptor homology (TIR) domain in its cytoplasmic region, TLR3 signals via the TIR domain-containing adapter inducing interferon- $\beta$ (TRIF). Then TRIF activates IRF3 and NF-kB via the interaction with tumor necrosis factor receptor associated factor 6 (TRAF6) and receptor interacting protein I (RIPI). This leads to the activation of genes encoding proinflammatory cytokines chemokine and interferon- $\beta$.

Toll like receptor 7 and 8 (TLR7, 8) which detect ssRNA rich in guanidine or uridine form a signal complex with Myeloid Differentiation 88 protein (MyD88), IL-1Receptor associated kinases 1 and 4 (IRAK1, 4) and TRAF6. TRAF6 will then activate interferon regulatory factor 7 (IRF7) through its ubiquitin E3 ligase. The IRF7 is then phosphorylated by a TBK1 independent pathway, and then translocates in the nucleus 
where it induces the expression of interferon- $\alpha$. TLR9 which signals after binding of DNA containing unmethylated cytosin-phosphatidyl-guanosine motifs (CpGDNA) uses the MyD88 pathway to activate IRF7. The phosphorylated IRF7 will then translocate to the nucleus where it activates the expression of genes encoding the interferon- $\alpha$.

All described pathways lead to the production of type I interferons. After secretion and binding to the IFN $\alpha / \beta$ receptor they activate the Jak/Stat signalling pathway, which leads to the expression of antiviral active factors.

To evade the host antiviral response induced by IFNs, most viruses have evolved strategies that antagonize this response by targeting steps that are essential for triggering host innate immunity [52]. NS1 protein of Influenza A virus [53] which can bind both ds RNA and single-stranded RNA with a non-modified triphosphate residue at the 5' end and the E3L protein of poxviruses $[54,55]$, which is a dsRNA binding protein constitute prominent examples of IFN antagonists.

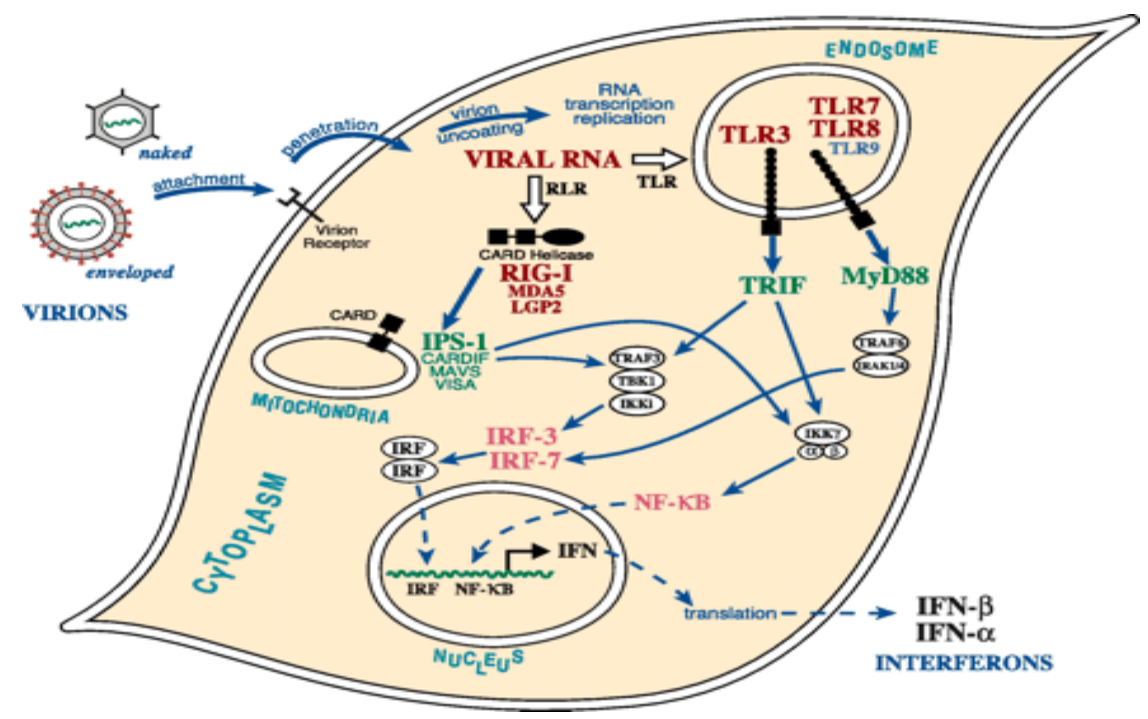

Figure 6. Signalling pathways activated by RNA leading to interferon production in virus-infected cells [56].

\subsubsection{Interference of RVFV with the IFN system}

Transcriptional activation of finely regulated inducible genes like the interferon $\beta$ (IFN- $\beta$ ) gene requires disruption of chromatin structure in order to allow the access of RNA polymerase to DNA [57]. The nucleosome is the basic unit of chromatin, consisting of DNA wrapped around an octamer of histones (two of each H2A, H2B, H3 and H4). This 
organized structure is a highly dynamic molecular edifice. The remodelling of it occurs in response to internal and external signals through post-translational modifications of histones, such as acetylation and methylation, as well as ATP-dependent nucleosome reorganization carried out by different types of multiprotein complexes. Promoter recruitment of chromatin remodelling complexes usually relies on transcription factors that bind to a specific DNA sequence and establish protein-protein interactions with chromatin remodelling complexes. Transcriptional regulation of the IFN- $\beta$ promoter requires specific binding of transcription factors as well as the orderly recruitment of chromatin remodelling complexes on the promoter region $[58,59]$. While the IFN- $\beta$ gene is constitutively repressed in non-infected cells, it is normally turned on as soon as a virus infects cells (Figure 6), establishing an antiviral state [60]. Interestingly, IFN- $\beta$-specific transcription factors such as IRF3, NF-KB and AP1 are initially activated in RVFVinfected cells [7]. RVFV-NSs however blocks IFN- $\alpha / \beta$ production in virus-infected cells at the transcriptional level. Unlike many other viral IFN antagonists, the IFN antagonistic activity of NSs stems from a general negative effect on the host cell transcriptional machinery rather than from interference with a component specific for the IFN system. The basic transcription factor TFIIH is the cellular target of RVFV-NSs. It is a multisubunit factor, composed of a core that contains the protein XPB, p62, p52, p44, and p34, is bridged by the XPD subunit to the CAK (cdk activating kinase), in which cdk7, cyclin H, and MAT1 are present [61]. TFIIH possesses several enzymatic activities: (1) the XPB helicase participates in promoter opening; (2) cdk7 phosphorylates RNA pol II as well as a number of transcriptional activators to control gene expression; and (3) XPD the second helicase, interacts with and is regulated by the p44 subunit of the TFIIH core. Disruption of XPD/p44 interaction inhibits the XPD helicase activity [62] and prevents accurate phosphorylation of certain nuclear receptors by CAK. This results in a decreased transactivation of genes under their control [63]. This is supported by the fact that NSs (i) was capable of blocking the IFN-inducing activity of a constitutively activated form of IRF-3, (ii) inhibited transcriptional activity of constitutive promoters, and (iii) prevented nonspecific upregulation by the general transcriptional activator [6]. 


\subsubsection{Dendritic cells}

DCs link the innate and the adaptive immune response and are key determinants of viral disease outcome. They are antigen presenting cells that initiate and regulate immunity [64]. They arise from different origins, are distributed in different tissues and express different surfaces receptors. DCs are found e. g. in skin and muscle where they pick up antigens, and then migrate from the peripheral tissues to the $\mathrm{T}$ cell areas of lymphoid organs to initiate immunity [65]. In lymphoid organs most DCs found arise from CD34 progenitor cells or blood monocytes in steady state [66]. These precursors can proliferate in the lymphoid organ, a process driven by the flt-3 ligand and Granulocyte macrophage colony stimulating factor (GM-CSF) [67].

Based on surface marker expression DCs are divided into distinct subsets [68]. Many of these markers are receptors involved in pattern recognition and antigen presentation. The DC subsets differ in immunomodulatory functions and preferentially react to distinct microbe stimuli [69]. Nevertheless, DCs share characteristic futures like production of high amounts of MHC class II, and potent T cell stimulating activity.

During viral infection DCs can perform multiple immunogenic tasks, including i) priming of naïve $\mathrm{T}$ cell by the expression of special costimulatory surface molecules; ii) cross presentation, that is, presentation of exogenous antigens in the context of MHC class I molecules to $\mathrm{CD} 8+\mathrm{T}$ lymphocytes, in addition to presentation of MHC class II restricted peptides; and iii) polarizing naïve T cells to various Th phenotypes.

\subsubsection{Plasmacytoid dendritic cells}

Plasmacytoid DCs (pDCs), which are derived from common lymphoid progenitor cells, express CD303, CD304, CD123 but not CD11c express Toll-like receptors TLR7 and TLR9 and respond to viral and self-nucleic acids with vigorous type I interferon production [70-73] therefore the term interferon producing cells (IPCs) is also used for this DC subset. pDCs also have special endosomal compartments for crosspresentation on MHC I [74]. The immediate production of IFN- $\alpha$ is a unique feature since all other cell types primarily respond to infections with IFN- $\beta$ production, which then triggers IFN- $\alpha$ synthesis. In most cell types viral RNAs are recognized by cytoplasmic RLRs. Additionally some cell types like conventional DCs (cDCs) express TLR3, which senses dsRNA. When these cells are infected with viruses or treated with either double stranded 
or 5' triphosphate single stranded RNA, IRF-3 is activated by phosphorylation through Tank binding kinase 1 (TBK-1), which leads to the production of IFN- $\beta$ (Figure 7).

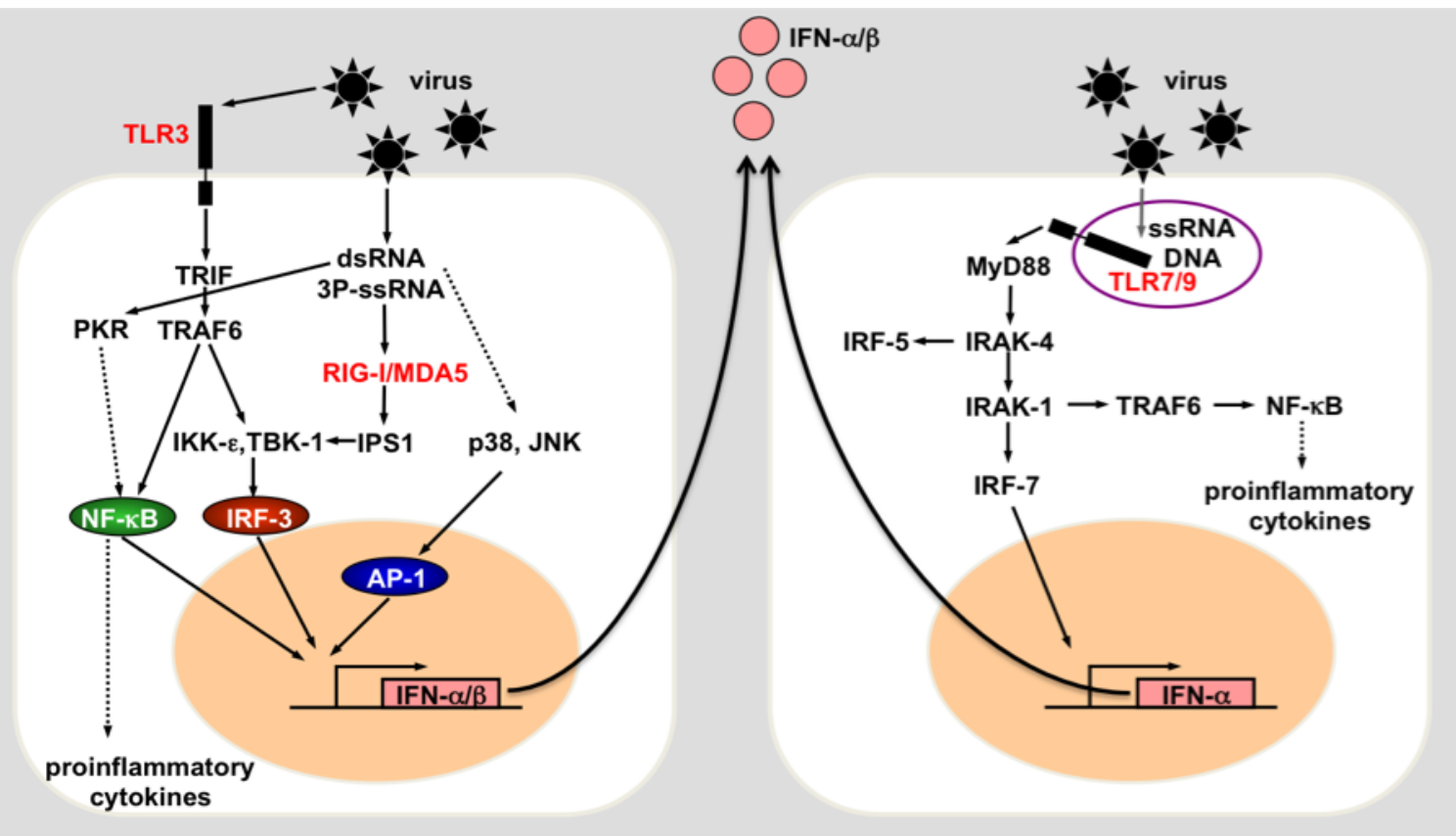

\section{most cell types including conventional DCs}

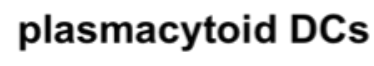

Figure 7. Virus-induced activation of primary IFN- $\alpha / \beta$ expression.

In pDCs however viral RNAs are sensed by TLR7, which leads to the activation of the MyD88 pathway in the cytosol, in turn following two ways. In one way the transcription factor IRF 7 which is (in contrast to other cells) constitutively expressed in pDCs is activated by phosphorylation through IRAK-1, which leads to the immediate production of IFN- $\alpha$. In a second way the transcription factor NF- $\kappa \mathrm{B}$ is activated through the tumor necrosis factor receptor associated kinase 6 (TRAF6), which induces the production of proinflammatory cytokines (Figure 7). Freshly isolated human pDCs are very poor inducers of $\mathrm{T}$ lymphocyte proliferation and it has been demonstrated that these pDCs present antigens inefficiently because they do not capture, process and load antigens onto MHC molecules as effectively as do other DC subsets [75]. More studies have shown that pDCs do not endocytose antigens as well as do other DC subsets [76]. In addition, pDCs show low expression of cathepsin $\mathrm{S}$ and cathepsin $\mathrm{D}$, which are lysosomal proteases involved in antigen processing [77]. In contrast to freshly isolated pDCs, activated pDCs augment cell surface expression of MHC class II and costimulatory molecules, increasing 
their $\mathrm{T}$ cell stimulatory ability $[76,78,79]$. Thus, activated pDCs present antigens and induce considerable expansion of $\mathrm{T}$ cell populations, although less efficiently than other DC subsets. pDCs also can cooperate with cDCs in vivo, inducing the differentiation of unpolarized antigen-experienced $\mathrm{T}$ cell populations that have been expanded by DCs [80, 81]. The independent and coordinated control of $\mathrm{T}$ cell proliferation and differentiation (Figure 8) may provide the immune system with greater flexibility in regulating immune responses.

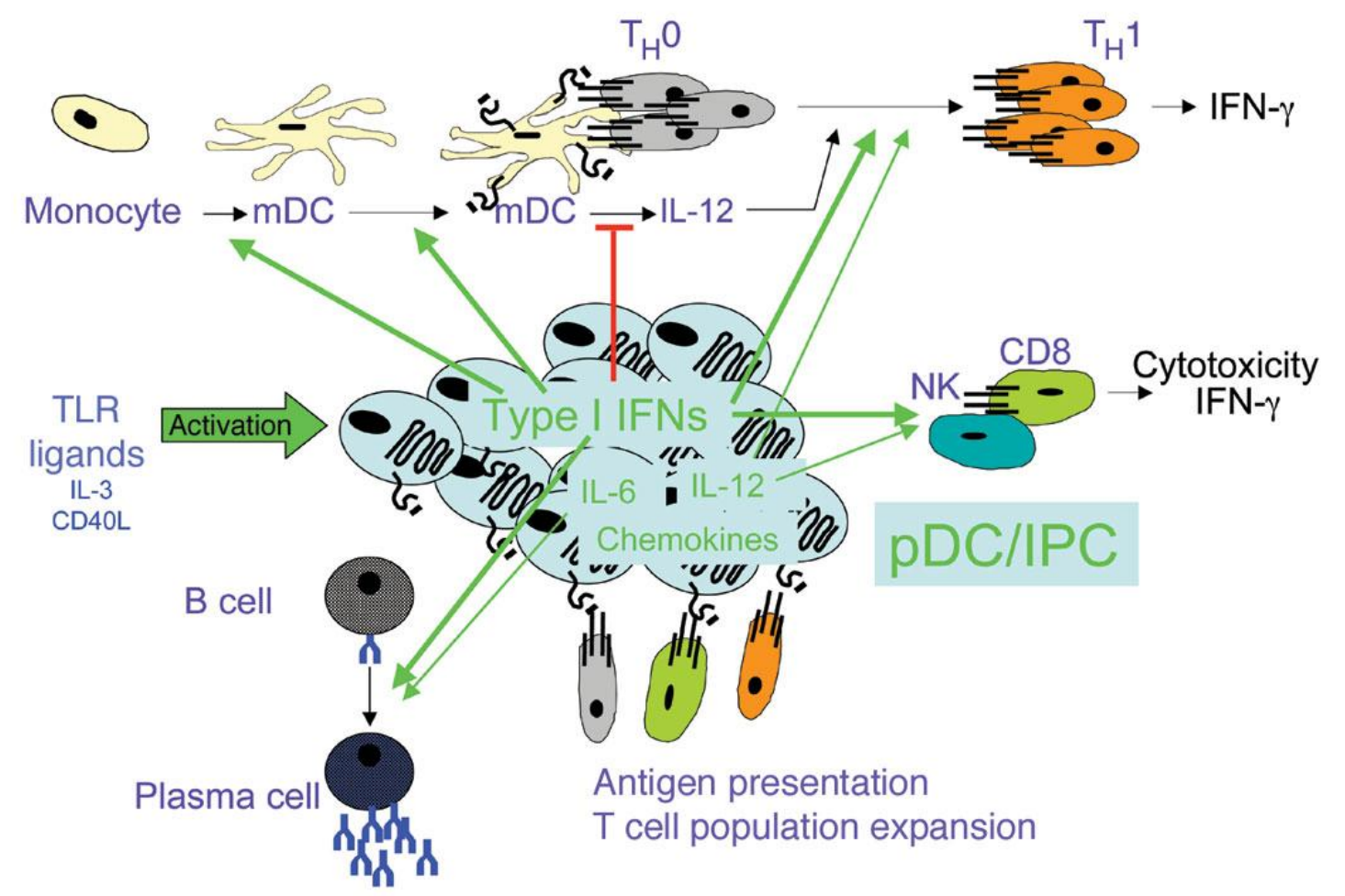

Figure 8. The immunostimulatory functions of activated pDCs. pDCs are mainly considered as Interferon producing cell (IPC). They are activated by Toll like receptor (TLRs), CD40L and in culture with IL-3. Production of type I interferon promotes Natural killer cell and CD8+ T cell cytotoxicity. Secretion of IFNgamma, interleukin12 (IL-12), proinflammatory chemokines, and interleukin 6 (IL-6). pDCs present antigens, expand memory and or naive $\mathrm{T}$ cells and then orchestrate the immune response. $\mathrm{T}$ helper 0 differentiated into T helper 1 through IL-12 activation will induce the production of interferon gamma [82].

\subsubsection{Conventional dendritic cells}

Conventional dendritic cells (cDCs), which are mostly derived from common myeloid progenitor cells, form many subsets (e.g. myloid DCs (mDCs)) and include migratory as well as lymphoid-resident dendritic cells that cooperate and are essential to one another to 
turn on a T cell response. cDCs are mostly devoted to taking up antigen in their steady state and presenting it to $\mathrm{T}$ cells in their activated or mature state. Early antigen presentation by lymphoid-resident DCs initiates activation and trapping of antigenspecific T lymphocytes in the draining lymph node, while migratory DCs interact with such $\mathrm{T}$ cells to induce clonal expansion [83]. In addition, cDCs can recognize viral RNA by endosomal TLR-3, [84] and the cytoplasmic receptors RIG-I, MDA5 (Figure 7) [85, 86].

After a viral infection in peripheral tissues cDCs will quickly sense the infection and process viral antigens. Peripheral dendritic cells from the skin, the lungs and the gut or genital mucosa, carry antigen into the lymph nodes where they appear as mature interdigitating dendritic cells within the $\mathrm{T}$ cell-dependent areas [87]. Interdigitating dendritic cells no longer process antigen but increase their $\mathrm{T}$ cell-stimulatory capacity by upregulating adhesion and costimulatory molecules [88, 89]. Intracellular MHC class II compartments will further release MHC class II-peptide complexes to the cell surface $[90,91]$. This results in a clonal expansion of cognate $\mathrm{T}$ cells and in the induction of $\mathrm{T}$ cell immunity, the T helper 1 (Th1) and T helper 2 (Th2) response. Another interaction for CTL responses needs essentially cooperation between antigen-specific Th1 and cytotoxic $\mathrm{T}$ cells. This is brought about by an antigen-loaded dendritic cell that displays antigens to both helper and cytotoxic T cells $[92,93]$. The first steps of DC activation can be bypassed by viral infection of dendritic cells [94]. Thereafter dendritic cells are not found in the efferent lymph probably due to virus-induced death in the lymph nodes. This elimination process might act as a feedback for removing the activation stimulus for immune responses $[95,96]$. Furthermore infected cDCs can act as Trojan horses which disseminate the virus in the infected host. The Human immunodeficient virus (HIV) system best illustrates the dual role of dendritic cells during virus infection. On the one hand, the uptake of virus, the processing of viral proteins and the presentation of viral antigens efficiently trigger both virus specific CD4+ and CD8+ T cell responses, whilst, at the same time, dendritic cells serve as vehicles to transport live virus into lymph nodes and thereby contribute to the transmission of virus to $\mathrm{T}$ cells and to the pathogenesis of the disease [96]. 


\subsubsection{Cytokine induced modulation of immune responses mediated by DCs}

In general cytokines modulate DCs as follows. DCs produce pro-inflammatory Interleukin $1 \beta$ (IL-1 $\beta$ ) [97] to increases the expression of adhesion factors on endothelial cells and therefore to support transmigration of leukocytes to the site of infection. Interleukin 6 (IL-6) acts mainly as a pro-inflammatory cytokine and is produced for example by Kupffer cells [98]. It affects changes in cell differentiation, growth, apoptosis and the induction of acute-phase responses [99]. It inhibits the generation of T regulatory cells (Treg) which hinder DCs and or normal CD4+T cells in their activities.

The pro-inflammatory chemokine Interleukin 8 (IL-8) is secreted by any cell with toll like receptors e.g. DC and mediates the inflammatory response, acts as chemoattractant and creates localized inflammation by oxidant stress [100]. The pro-inflammatory cytokine Tumor necrosis factor alpha $(\mathrm{TNF} \alpha)$ is involved in systemic inflammation that stimulates the acute phase reaction and is also produced by DCs.

The proinflammatory cytokine Interleukin 12 (IL-12) naturally produced by dendritic cells [101], acts as T cell stimulating growth factor, and is involved in the differentiation of naive T cells into Th0 cells, which will further develop into either Th1 cells or Th2 cells. It also mediates enhancement of the cytotoxic activity of NK cells and CD8+ cytotoxic T lymphocytes. The anti-inflammatory cytokine Interleukin 10 (IL-10) displays potent abilities to suppress the antigen presentation capacity of antigen presenting cells.

\subsection{Aim of the study}

In recent work it has been shown that the RVFV protein NSs, a non-structural protein, has an inhibitory effect on the interferon response of the innate immune system [6]. The immune compromising effect of wtRVFV strains through NSs-mediated interference with the innate immune system is an important virulence factor. However the IFN-antagonistic function of NSs was only studied in non-immune cells so far and nothing is known about the potential immunomodulatory functions of NSs in immune cells.

This project has two concurrent primary objectives:

- $\quad$ First to elucidate if RVFV field isolates differ in their efficiency to subvert type I IFN induction in non-immune cells due to variations of NSs. Different RVFV 
strains vary significantly in their NSs sequences and possible functional consequences have not been investigated yet

- Second to shed light on the immune evasion mechanisms of RVFV strains in immune cells. Since dendritic cells are key components of the adaptive immune system and additionally represent the main link between innate and adaptive immune system dendritic cells were chosen as a model system. pDCs and mDCs use different pathways to sense viral infections and therefore it is of great interest to investigate if possible differences in their response to RVFV infections exist. 


\section{Materials and Methods}

\subsection{Materials}

\subsubsection{Bacterial strains}

The bacterial strains listed in Table 2 were used for cloning purposes.

Table 2. Bacterial strains

\begin{tabular}{|c|c|c|}
\hline Strain & Genotype & Source/ Manufacturer \\
\hline $\mathrm{INV} \alpha \mathrm{F}^{\prime}$ & $\begin{array}{l}\mathrm{F}^{\prime} \quad \text { endA1 } \operatorname{rec} \mathrm{A} 1 \quad h s d \mathrm{R} 17 \quad(\mathrm{rk}-, \quad \mathrm{mk}+) \\
\text { supE44 thi-1 } \operatorname{gyr} \mathrm{A} 96 \text { relA1 } \varphi 80 \text { lacZ } \Delta \mathrm{M} 15 \\
\Delta(\text { lacZYA-argF)U169 } \lambda \text { - }\end{array}$ & $\begin{array}{l}\text { Invitrogen } \mathrm{GmbH} \text {, Karlsruhe, } \\
\text { Deutschland }\end{array}$ \\
\hline XL1-Blue & $\begin{array}{l}\text { recAl endAl gyrA96 thi-1 hsdR17 supE44 } \\
\text { relAl lac }\left[\mathrm{F}^{\prime} \text { proAB lacIqZDM15 Tn10 }\right. \\
\text { (Tetr) }]^{\mathrm{c}}\end{array}$ & $\begin{array}{l}\text { Stratagene Europe, } \\
\text { Amsterdam, The Netherlands }\end{array}$ \\
\hline
\end{tabular}

\subsubsection{Plasmids}

Plasmids used are listed in Table 3. Every listed plasmid has an ampicillin resistance gene for selection in E. coli.

Table 3. Plasmids

\begin{tabular}{lll}
\hline Plasmids & Description & Source \\
pCRII & TA-Cloning vector & $\begin{array}{l}\text { Invitrogen GmbH, Karlsruhe, } \\
\text { Germany }\end{array}$ \\
p125-luc & This is a reporter plasmid for $\begin{array}{l}\text { Takashi Fujita, The Tokyo } \\
\text { monitoring IFN- } \beta \text { promoter activity. Metropolitan Institute of Medical }\end{array}$ \\
& It contains the firefly luciferase Sciences, Tokyo, Japan \\
& cDNA under the control of the human \\
& IFN- $\beta$ promoter \\
pI.18 & Empty eukaryotic expression vector, Jim Robertson, National Institute \\
& carries the constitutively active for Biological Standards and \\
& hCMV IE-promoter and the hCMV Control, Hertfordshire, United \\
& intron A followed by a multiple Kingdom \\
& cloning site for the insertion of cDNA \\
& and an hCMV polyA signal.
\end{tabular}




\begin{tabular}{ll}
\hline pRL-SV40 vector & pRL-SV40 is a reporter vector Promega, Mannheim, Germany. \\
& containing a cDNA encoding Renilla \\
& luciferase downstream of a SV40 \\
& early enhancer/promoter region and \\
& provides strong, constitutive \\
& expression of (Rluc) in a variety of \\
& mammalian cells [102]. The vector is \\
& used as an internal control. \\
\end{tabular}

\subsubsection{Viruses and cells}

Viruses and cells were provided and produced as described in Table 4.

Table 4. Sources of viruses and cells

\begin{tabular}{lll}
\hline Name & Source & Production
\end{tabular}

Wild type Rift Valley fever Department of Virology in virus strain ZH548 (wtRFVV Göttingen, [12, 103]

ZH548)

Rift Valley fever virus, strain

clone 13

Vero E6 cells
Source

Viruses were produced under

BSL3 conditions by infecting

Vero cells at MOI of $10^{-3}$ and

harvesting the medium at $72 \mathrm{~h}$

p.i. These stocks are kept at -

$80^{\circ} \mathrm{C}$ until use.

Institute for Microbiology of (see chapter 2.2.4.3)

the Armed Force, München,

Germany

Human primary plasmacytoid Deutsches Rotes Kreuz

Freshly produce in situ from

dendritic cells

(German Red Cross at Springe, healthy human donor buffy

Germany)

coat

(see chapter 2.2.4.1)

Human primary conventional Deutsches Rotes Kreuz

Freshly produce in situ from

dendritic cells

(German Red Cross at Springe, healthy human donor buffy

Germany) 


\subsubsection{Enzymes}

Enzymes used and the manufacturers of the enzymes are listed in Table 5.

Table 5. Enzymes

\begin{tabular}{|c|c|c|}
\hline Name & & Manufacturer \\
\hline T4-DNA-Ligase & & Roche GmbH, Mannheim, Germany \\
\hline Taq-DNA-Polymerase & & 5-Prime GmbH, Hamburg, Germany \\
\hline$P f u$-Ultra ${ }^{\mathrm{TM}} \quad$ II $\quad$ Fusion & DNA- & Stratagene Europe, Amsterdam, The Netherlands \\
\hline \multicolumn{3}{|l|}{ Polymerase } \\
\hline EcoRI & & Fermentas GmbH, Leon-Rot, Germany \\
\hline BglII & & Fermentas GmbH, Leon-Rot, Germany \\
\hline SalI & & Fermentas GmbH, Leon-Rot, Germany \\
\hline Antarctic Phosphatase & & $\begin{array}{l}\text { New England Biolabs GmbH, Frankfurt am Main, } \\
\text { Germany }\end{array}$ \\
\hline
\end{tabular}

\subsubsection{Reagents and Kits}

The kits listed in Table 6 were used for DNA manipulation, chemiluminescence detection, dendritic cell purification and FACS analysis.

Table 6. Reagents and Kits

\begin{tabular}{ll}
\hline Name & Manufacturer \\
\hline DNA Clean\& Concentrator ${ }^{\mathrm{TM}}-5$ & Zymoclean Research Corporation, Orange, USA \\
Dual Promotor TA Cloning® Kit & Invitrogen GmbH, Karlsruhe, Germany \\
$\begin{array}{l}\text { Dual Luciferase Reporter } \\
\text { System }\end{array}$ & Promega, Mannheim, Germany. \\
$\begin{array}{ll}\text { Human Inflammatory cytokines } \\
\text { Kit (Cytometric bead array, CBA) }\end{array}$ & BD Biosciences, Heidelberg, Deutschland \\
Human IFN- $\alpha$ Elisa Kit & PBL InterferonSource, Piscataway, USA \\
peq GOLD Plasmid Miniprep Kit I & PEQLAB Biotechnologie GmbH, Erlangen, Germany \\
Rapid DNA Ligation Kit & Roche Diagnostics GmbH, Mannheim, Germany
\end{tabular}


RNeasy®Mini kit

Z-Competent ${ }^{\mathrm{TM}}$ E. coli

Transformation Kit \& Buffer

Zymoclean $^{\mathrm{TM}}$ Gel DNA Recovery

Kit

Nuclebond AX100 Plasmid

Preparation Kit

NucleoSpin Gel Extraction Kit

Plasmacytoid dendritic cell

isolation Kit human

Myeloid dendritic cell isolation Kit human

FuGene ${ }^{\circledR}$ HD Transfection reagent
Qiagen GmbH, Hilden, Germany

Zymoclean Research Corporation, Orange, USA

Zymoclean Research Corporation, Orange, USA

Macherey-Nagel GmbH, Düren, Germany

Macherey-Nagel GmbH, Düren, Germany

Milteny Biotechnology GmbH, Bergisch Gladbach, Germany

Milteny Biotechnology GmbH, Bergisch Gladbach, Germany

Roche GmbH, Mannheim, Germany

\subsubsection{Antibodies}

The antibodies used for the detection experiments are listed in Table 7.

Table 7. Antibodies

\section{Name Manufacturer}

Anti human CD1c antibody BD Pharmingen ${ }^{\mathrm{TM}}$ Biosciences, San Diego, California, labeled with (PECy ${ }^{\mathrm{TM}} 5$, APC, USA

FITC)

Anti human CD11c antibody BD Pharmingen Biosciences, San Diego, California, USA labeled with (PE Cy $\left.{ }^{\mathrm{TM}} 7\right)$

Anti human CD83 Antibody BD Pharmingen Biosciences, San Diego, California, USA labeled with (PE, FITC, APC, PE

Cy ${ }^{\mathrm{TM}} 5$ )

Anti human CD80 antibody BD Pharmingen Biosciences, San Diego, California, USA labeled with (PE Cy ${ }^{\mathrm{TM}} 5$, PE,

FITC) 
Anti human CD86 antibody BD Pharmingen Biosciences, San Diego, California, USA labeled with (PE, FITC, PE Cy'M5,

APC)

Anti human CD54 antibody BD Pharmingen Biosciences, San Diego, California, USA labeled with (APC, PE CyTM, PE)

Anti human CD58 antibody BD Pharmingen Biosciences, San Diego, California, USA labeled with (PE, PE $\mathrm{Cy}^{\mathrm{TM}} 5$,

FITC)

Anti human CD40 antibody

labeled with (APC, PE Cy ${ }^{\mathrm{TM}} 5$, PE,

FITC)

Anti human CD40 antibody BD Pharmingen Biosciences, San Diego, California, USA labeled with (APC, PE Cy ${ }^{\mathrm{TM}} 5$, PE,

FITC)

Anti human CD123 antibody BD Pharmingen Biosciences, San Diego, California, USA labeled with (PE Cy ${ }^{\mathrm{TM}} 5$, PE, FITC,

PERCP-Cy5.5)

Anti human CD14 antibody BD Pharmingen Biosciences, San Diego, California, USA labeled with (Pacific blue, APC,

APC-Cy'M

Anti human MHCI antibody BD Pharmingen Biosciences, San Diego, California, USA labeled with (PE-Cy'

Anti human MHCII antibody BD Pharmingen Biosciences, San Diego, California, USA labeled with (Pacific blue)

Anti FLAG mouse monoclonal Sigma-Aldrich, Deisenhofen, Germany

antibody, clone M2

Anti mouse IgG labelled with Invitrogen GmbH, Karlsruhe, Germany

Alexa Fluor 555 


\subsubsection{Media and Buffers}

Table 8 lists all media and buffers used in the experimental procedures.

Table 8. Media and Buffers

\begin{tabular}{|c|c|}
\hline Name & Composition \\
\hline Agarose gel [1\%] & $\begin{array}{l}100 \mathrm{ml} \text { TAE }[1 \mathrm{x}], 1 \mathrm{~g} \text { agarose, } 5 \mu \mathrm{l} \text { ethidium bromide } \\
\text { solution (final concentration } 0,5 \mathrm{mg} / \mathrm{ml} \text { ) }\end{array}$ \\
\hline Ethidium bromide solution & $10 \mathrm{mg} / \mathrm{ml}$ in $\mathrm{H}_{2} \mathrm{O}$ \\
\hline $\begin{array}{l}\text { Dulbecco's modified Eagle's } \\
\text { medium (DMEM) }\end{array}$ & C.C.pro GmbH Neustadt, Germany \\
\hline OptiMEM & Invitrogen $\mathrm{GmbH}$, Karlsruhe, Germany \\
\hline Fetal Calf Serum (FCS) & Biochrom AG, Berlin, Germany \\
\hline LB-Agar with ampicillin & $\begin{array}{l}10 \mathrm{~g} \text { Bacto- Tryptone, } 5 \mathrm{~g} \text { Bacto- Yeast Extract, } 10 \mathrm{~g} \mathrm{NaCl} \text {, } \\
1 \mathrm{ml} \text { Ampicillin }[100 \mu \mathrm{g} / \mathrm{ml}], 15 \mathrm{~g} \mathrm{Bacto}^{\mathrm{TM}}-\text { Agar, ad } 11 \\
\mathrm{H}_{2} \mathrm{O}\end{array}$ \\
\hline LB-Medium with ampicillin & $\begin{array}{l}10 \mathrm{~g} \text { Bacto- Tryptone, } 5 \mathrm{~g} \text { Bacto- Yeast Extract, } 10 \mathrm{~g} \mathrm{NaCl} \text {, } \\
1 \mathrm{ml} \text { Ampicillin }[100 \mu \mathrm{g} / \mathrm{ml}] \text {, ad } 11 \mathrm{H}_{2} \mathrm{O}\end{array}$ \\
\hline Phosphate-buffered Saline (PBS) & C.C.pro GmbH Neustadt, Germany \\
\hline $\begin{array}{l}\text { Roswell Park Memorial Institute } \\
1640 \text { medium (RPMI 1640) }\end{array}$ & C.C.pro GmbH Neustadt, Germany \\
\hline S.O.C. Medium & Invitrogen GmbH, Karlsruhe, Deutschland \\
\hline $\mathrm{TAE}[1 \mathrm{x}]$ & $40 \mathrm{ml}$ TAE [50x] ad $1960 \mathrm{ml} \mathrm{H}_{2} \mathrm{O}$ \\
\hline TAE $[50 x]$ & $\begin{array}{l}242 \mathrm{~g} \text { Tris- } \mathrm{HCl}, 57,1 \mathrm{ml} \text { acetic acid, } 100 \mathrm{ml} \text { EDTA pH8, } 0 \\
{[0,5 \mathrm{M}] \text { adjusted to } 1000 \mathrm{ml} \text { with } \mathrm{H}_{2} \mathrm{O}}\end{array}$ \\
\hline Trypsin-EDTA w/o Ca++, $\mathrm{Mg}++$ & Biochrom AG, Berlin, Germany \\
\hline
\end{tabular}




\subsubsection{Chemicals}

Chemicals used in the experimental procedures are listed in Table 9.

Table 9. Chemicals

\begin{tabular}{|c|c|}
\hline Substances & Manufacturer \\
\hline Antifect FD10 & Schülke \& Meyer GmbH, Norderstedt, Germany \\
\hline Agarose & Biozym Scientific GmbH, Oldendorf, Germany \\
\hline Ampicillin & Roche Diagnostics GmbH, Mannheim, Germany \\
\hline Bacto-Agar & BD Diagnostics, Heidelberg, Germany \\
\hline Bacto-Yeast extract & BD Diagnostics, Heidelberg, Germany \\
\hline Bacto-Tryptone & BD Diagnostics, Heidelberg, Germany \\
\hline $\begin{array}{l}\text { Desoxyribonucleoside triphosphate } \\
\text { (dNTP) }\end{array}$ & Fermentas GmbH, Leon-Rot, Germany \\
\hline $\begin{array}{l}\text { DNA-Ladder O'GeneRuler }(1 \mathrm{~kb} \text {, } \\
100 \mathrm{bp})\end{array}$ & Fermentas GmbH, Leon-Rot, Germany \\
\hline Acetic acid & Merck KG aA, Darmstadt, Germany \\
\hline Ethanol & Merck KG aA, Darmstadt, Germany \\
\hline Ethidium bromide & Carl Roth GmbH + Co. KG, Karlsruhe, Germany \\
\hline $\begin{array}{l}\text { Ethylene-diamine-tetra-acetate } \\
\text { (EDTA) }\end{array}$ & Serva Electrophoresis GmbH, Heidelberg, Germany \\
\hline FACS-Flow & BD Biosciences, Heidelberg, Germany \\
\hline Fluosave mounting medium & Calbiochem, Bad Soden, Germany \\
\hline GM-CSF (Leukomax) & Novartis Pharma, Basel, Switzerland \\
\hline Hydrochloric acid & Merck KG aA, Darmstadt, Germany \\
\hline Interleukin-3 & BD Pharmingen Biosciences, San Diego, California \\
\hline Interleukin-4 & Cellgenix, Freiburg, Germany \\
\hline Interleukin-6 & R\&DSystems GmbH, Wiesbaden-Nordenstadt, Germany \\
\hline Orange Loading Dye (6x) & Fermentas GmbH, Leon-Rot, Germany \\
\hline
\end{tabular}


Polyethylenglycol-Sorbitan-

Monolaurat Tween ${ }^{\circledR} 20$

Tris-base

Tris- $\mathrm{HCl}$

Triton X-100

(Octylphenol-Polyethylenglycol)
Sigma- Aldrich Chemie GmbH, Steinheim, Germany

Carl Roth GmbH + Co. KG, Karlsruhe, Germany

Carl Roth GmbH + Co. KG, Karlsruhe, Germany

Serva, Heidelberg, Germany

\subsubsection{Stock solutions}

A comprehensive overview over stock solutions used throughout the experimental procedures is given in Table 10 .

Table 10. Stock solutions

\section{Stock solutions}

- dNTP stock solution $10 \mathrm{mM}$ of each dNTP in $\mathrm{H}_{2} \mathrm{O}$, stored at $-20^{\circ} \mathrm{C}$

- EDTA stock solution $500 \mathrm{mM}$ EDTA dissolved in $\mathrm{H}_{2} \mathrm{O}$ with addition of solid $\mathrm{NaOH}$ adjusted to $\mathrm{pH} 8.0$

- Ethidium bromide stock solution $10 \mathrm{mg} / \mathrm{ml}$ Ethidium bromide

- PBS-Buffer (1x) 10 mM Na2HPO4, 1.8 mM KH2PO4 pH 7.3, 140 mM NaCl, 2.7 mM $\mathrm{KCl}$

- Paraformaldehyde solution (4\%PFA): $40 \mathrm{~g}$ of PFA (Merck, Germany) were dissolved in $500 \mathrm{ml}$ distilled water, heated to $60^{\circ} \mathrm{C}$. Aldehyde formation occurred upon addition of few drops of $\mathrm{NaOH}(1 \mathrm{M})$. Then the solution was cooled to room temperature, filtered and mixed with $500 \mathrm{ml}$ of $0.2 \mathrm{M}$ PB to prepare 1 litter of $4 \%$ PFA $(\mathrm{pH}=7.4)$.

- Ampicillin stock solution 100 mg/ml Ampicilin (Na-Salt)

- Antifect FD19: $2 \%$ and 3\% in $\mathrm{H}_{2} \mathrm{O}$ 


\subsubsection{Laboratory equipment}

Equipment as listed in Table 11 was used.

Table 11. Laboratory equipment

\begin{tabular}{ll}
\hline Equipment & Manufacturer \\
\hline Incubator for bacterial cultures & Heraeus Instruments GmbH, Hannover, Germany \\
Chemidoc XRS System & Bio Rad Laboratories GmbH, München, Germany \\
FACS Canto II & BD Biosciences, Heidelberg, Germany \\
Freezer $\left(-20^{\circ} \mathrm{C}\right)$ & Liebherr GmbH, Ochsenhausen, Germany \\
Freezer $\left(-80^{\circ} \mathrm{C}\right)$ & Thermo Scientific, Rockford, USA \\
Freezer $\left(-140^{\circ} \mathrm{C}\right)$ & Thermo Scientific, Rockford, USA \\
Gel electrophoresis chamber & Invitrogen Corporation, California, USA
\end{tabular}

Electrophoresis power ECPS Pharmacia Healthcare, Uppsala, Sweden 3000/150 GE

Electrophoresis Power LNGs 350- Heinzinger, Rosenheim, Germany 06

Heating Block (Thermomixer)

Fridge $\left(5^{\circ} \mathrm{C}\right)$

Nano Drop ND-1000

Spectrophotometer

Laminar Flow Hood

Thermocycler

Thermomixer compact

Vortex Genie 2 ${ }^{\mathrm{TM}}$

Precision Balances L 420 P

Precision Balances U 4800 P

Rocker

Centrifuge (Multifuge 3 L-A)
Eppendorf AG, Hamburg, Germany

Liebherr GmbH, Ochsenhausen, Germany

PeQlab Biothecnology GmbH Erlangeg, Germany

Heraeus Sepatech GmbH, Osterode, Germany

Biometra GmbH, Göttingen, Germany

Eppendorf AG, Hamburg, Germany

Bender \& Hobein AG, Zürich, Switzerland

Sartorius, Stedim biotech GmbH, Göttingen, Germany

Sartorius, Stedim biotech GmbH, Göttingen, Germany

Schuett24 GmbH, Göttingen, Germany

Heraeus Thermo Scientific, Bonn, Germany 
Centrifuge (Megafuge 1.0R)

BSL3 lab facilities

Shaking Water Bath Julabo S.W22 JULABO Labortechnik GmbH Seelbach, Germany

\subsubsection{Disposables}

A diverse range of plastic materials and other materials was used and is listed in Table 12.

Table 12. Other materials

Material Manufacturer

96 well microplate, tissue culture Greiner Bio-One GmbH, Frickenhausen, Germany treated

Greiner Bio-One GmbH, Frickenhausen, Germany

6well, 12well, 24well plate, tissue

culture treated

Falcon-Tubes $(15 \mathrm{ml}, 50 \mathrm{ml})$

Gloves - Latex

Gloves - Nitril

Delicate Task Wipes

Tubes $(1,5 \mathrm{ml}$ and $2,0 \mathrm{ml})$

Parafilm

PCR-Tubes $(0,2 \mathrm{ml})$

Serological Pipettes

(1 ml, $2 \mathrm{ml}, 5 \mathrm{ml}, 10 \mathrm{ml}, 25 \mathrm{ml})$

Pipette-Tips with Filters (0,1- Starlab GmbH, Ahrensberg, Germany

$10 \mu \mathrm{l}, 1,0-100 \mu \mathrm{l}, 100-1000 \mu \mathrm{l})$

Pipette-Tips without Filters (0,1- Starlab GmbH, Ahrensberg, Germany

$10 \mu \mathrm{l}, 1,0-100 \mu \mathrm{l}, 101-1000 \mu \mathrm{l})$

Safe-Lock-Tubes

Eppendorf AG, Hamburg, Germany

$(0,5 \mathrm{ml}, 1,5 \mathrm{ml}, 2 \mathrm{ml})$

Strep-Tactin-Sepharose Columns
Sarstedt AG \& Co, Nümbrecht, Germany

Mikroflex Corporation, Vienna, Austria

GE Healthcare Europe NV, Brussel, Belgium

Kimberly-Clark Europe Limited, Kings Hill, UK

Sarstedt AG \& Co, Nümbrecht, Germany

American National Can, Chicago, USA

Biozym, Scientific GmbH, Oldendorf, Germany

Greiner Bio-One GmbH, Frickenhausen, Germany 
Autoclaving Bags

Lab Logistic Group GmbH, Meckenheim, Germany

(300mmx200mm)

\subsubsection{Computer software}

The following software was used as listed in Table 13 .

Table 13. Computer software

\begin{tabular}{lll}
\hline Name & Manufacturer & Use \\
\hline Microsoft Office 2007 & Microsoft Inc. & Text and Data Processing \\
Adobe-Photoshop & Adobe Systems Inc. & Image Processing \\
EndNote X2 & Thomson Reuters & Reference Management \\
Lasergene 7.1 & DNASTAR Inc. & $\begin{array}{l}\text { DNA Sequence Analysis and } \\
\text { Vector Construction }\end{array}$ \\
$\begin{array}{l}\text { BD FACSDiva } \\
\text { Version 6.1.2 }\end{array}$ & BD Biosciences & $\begin{array}{l}\text { Recording and controlling flow } \\
\text { cytometric measurements and } \\
\text { primary data analysis. }\end{array}$ \\
\hline $\begin{array}{l}\text { Flow Jo } \\
\text { Ashland }\end{array}$ & $\begin{array}{l}\text { Flow-cytometry primary data } \\
\text { analysis. }\end{array}$ \\
\hline $\begin{array}{l}\text { FCAP Array } \\
\text { TM software }\end{array}$ & Soft Flow Hungary Ltd. & $\begin{array}{l}\text { Flow-cytometry primary data } \\
\text { analysis. }\end{array}$ \\
Chemidoc software & Quantity One 1-D & $\begin{array}{l}\text { Gel analysis } \\
\text { Analysis Sofware } \\
\text { version 4.6.1. Bio-Rad }\end{array}$ \\
\hline
\end{tabular}




\section{$2.2 \quad$ Methods}

\subsubsection{Manipulation of nucleic acid}

\subsubsection{1 cDNA preparation}

Total RNA was prepared from virus culture supernatants of strains as previously described [104]. After RNA extraction, the complementary DNA (cDNA) was prepared for each strain. The first strand cDNA synthesis was performed using Superscript III (Invitrogen, Leek, The Netherlands). For cDNA synthesis 50 to $100 \mathrm{ng} / \mu \mathrm{l}$ RNA template were mixed with 100ng of random primer and $10 \mathrm{mM}$ of dNTP in a total volume of $14 \mu 1$. After denaturation at $65^{\circ} \mathrm{C}$ for 5 minutes using a Biometra cycler the RNA was placed in ice for one minute. Then a reaction mix was prepared using 50 to $100 \mu \mathrm{g}$ of total RNA from the previous step, $10 \mu \mathrm{M}$ DTT and $200 \mathrm{U}$ of superscript III RT in a total volume of $20 \mu \mathrm{l}$. The cDNA synthesis was performed at $25^{\circ} \mathrm{C}$ for $5 \mathrm{~min}$, followed by $55^{\circ} \mathrm{C}$ for $60 \mathrm{~min}$ and finally $70^{\circ} \mathrm{C}$ for $15 \mathrm{mn}$. The cDNAs obtained were stored at $-80{ }^{\circ} \mathrm{C}$ until used.

\subsubsection{Amplification of cDNA and preparation of PCR product for ligation}

The cDNAs $(1,5 \mu \mathrm{l})$ were used as templates in PCR using AccuPrime Pfx DNA polymerase (Invitrogen) with RVFV-NSs forward primer which adds a 5' BglII restriction site (5'GACAGAAGATCTATGGATTACTTTCCTGTGATATCTG3') and RVFV-NSs reverse primer

(5'GTCGACTCACTTGTCATCGTCGTCCTTGTAGTCATCAACCTCAACAAATCCA TC3'), which carries an immunogenic FLAG-tag as well as a 3' Sall restriction site in a total volume of $50 \mu \mathrm{l}$. The addition of the FLAG-tag at the C-terminus of RVFV-NSs neither affects the IFN-antagonistic function of NSs nor the ability to form nuclear filaments [7].

The temperature profile consisted of a denaturation step at $95{ }^{\circ} \mathrm{C}$ for $120 \mathrm{~s}$, and $10 \mathrm{PCR}$ cycles of denaturation at $95{ }^{\circ} \mathrm{C}$ for $30 \mathrm{~s}$, annealing for $30 \mathrm{~s}$ with an initial temperature of $57{ }^{\circ} \mathrm{C}$ which decreased by $0.5^{\circ} \mathrm{C}$ for each cycle and an extension step at $68{ }^{\circ} \mathrm{C}$ for $60 \mathrm{~s}$. Then, 25 PCR cycles were performed with a constant annealing temperature of $52{ }^{\circ} \mathrm{C}$ while the other parameters remained unchanged. A final extension step was performed at $68{ }^{\circ} \mathrm{C}$ for $300 \mathrm{~s}$ followed by cooling. 


\subsubsection{Agarose gel electrophoresis of PCR products}

The amplicons were separated in an electrophoresis chamber at 90 volts, using a $0,8 \%$ agarose gel containing $0,05 \%$ ethydium bromide. The PCR product was extracted from the gel and the DNA recovered using the peqGOLD Plasmid Gel Extraction Miniprep kit (Table 6).

\subsubsection{Cloning experiments}

\subsubsection{Dephosphorylation of plasmids}

The reaction mix for the dephosphorylation was prepared using $97 \mathrm{ng} / \mu \mathrm{l}$ of $\mathrm{pI} .18(1 \mu \mathrm{l})$, Antarctic phosphatase buffer $(2 \mu \mathrm{l}), 100 \mathrm{Units} / \mu \mathrm{l}$ of antarctic phosphatase $(1 \mu \mathrm{l})$, bidistilled water $(6,7 \mu 1)$ in a total volume of $20 \mu 1$.

This reaction mix was first incubated at $37^{\circ} \mathrm{C}$ for 15 minutes followed by an inactivation step at $65^{\circ} \mathrm{C}$ for 5 minutes.

\subsubsection{Ligation of PCR products into PCRII vector}

TA cloning of purified PCR products into the vector pCRII was performed according to the manufacturer's instructions (Invitrogen).

To add A overhangs to the PCR amplificates, Platinum Taq DNA-polymerase $(0,5 \mu \mathrm{l})$, dNTPs ( $1 \mu \mathrm{l}$ of a $50 \mathrm{mM}$ solution), a PCR buffer $(2 \mu \mathrm{l})$ and distilled $(10,5 \mu \mathrm{l})$ water were added to PCR product $(6 \mu \mathrm{l})$ in a total volume of $20 \mu \mathrm{l}$ and incubated for 15 minutes at $72^{\circ} \mathrm{C}$.

For TA ligation $4 \mu \mathrm{l}$ out of $20 \mu \mathrm{l}$ of the above mix (containing 300-500 ng PCR products with A overhangs) was used for ligation into pCR II vector using the TA-cloning kit according to the manufacturer's instructions (Table 6).

\subsubsection{Transformation of $\mathrm{pCRII}$ vector}

For TA cloning about 2 to $5 \mu$ l of the ligation mix (Chapter 2.2.2.3) was added to $50 \mu \mathrm{l}$ INV $\alpha F^{\prime}$ bacteria (One Shot INV $\alpha F^{\prime}$ competent cells, (Table 2) and incubated on ice for $30 \mathrm{~min}$. A heat shock was performed at $42^{\circ} \mathrm{C}$ for 30 seconds before $250 \mu \mathrm{l}$ of SOC-media was added and the mix was incubated for 1 hour at $37^{\circ} \mathrm{C}$ and $300 \mathrm{RPM}$.

The mixture was plated out on plates containing Luria-Bertani Medium (LB) Ampicillin inoculated with $40 \mu \mathrm{l}$ of $40 \mu \mathrm{g} \mathrm{X}-\mathrm{Gal}$ and the plates were incubated overnight at $37^{\circ} \mathrm{C}$. 
Colonies carrying recombinant plasmids with an insertion disrupting the LacZ gene formed white colonies, those with no insertion formed blue colonies. White colonies were picked and transferred to $3 \mathrm{ml} \mathrm{LB}$ medium after $24 \mathrm{~h}$ post incubation. The control plates were checked for background ligation of empty plasmids.

\subsubsection{Ligation of PCR products into pI.18}

To prepare the plasmid for ligation, pI.18, was double digested as described in Chapter 2.2.2.7 and dephosphorylated as described in chapter 2.2.2.1.

Three reactions mix were prepared for the ligation. The first control mix contained phosphorylated pI.18 vector $(1 \mu \mathrm{l})$, in a $20 \mu \mathrm{l}$ ligation volume containing Buffer $5 \mathrm{x}(4 \mu \mathrm{l})$, ligase $(1 \mu \mathrm{l})$ completed with distilled water of the Rapid DNA Ligation kit.

The second control mix contained, dephosphorylated pI.18 vector $(2 \mu 1)$, in a $20 \mu 1$ ligation volume containing Buffer $5 \mathrm{x}(4 \mu \mathrm{l})$, ligase $(1 \mu \mathrm{l})$ completed with distilled water.

The third ligation mix contained dephosphorylated pI.18 vector $(1 \mu 1)$, RVFV NSs insert

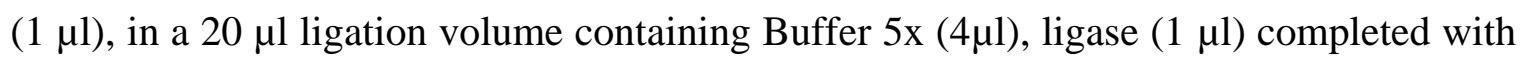
distilled water.

\subsubsection{Transformation of pI.18}

The pI.18 ligations were transformed into XL I Blue using Z competent transformation kit (Table 6). $5 \mu$ l of the ligation mixture (Chapter 2.2.2.3) was transferred in $50 \mu$ l of XLI Blue cells, and incubated on ice for $30 \mathrm{~min}$ heat shock at $42^{\circ} \mathrm{C}$.

The cells were spread onto LB-Amp plates and incubated at $37^{\circ} \mathrm{C}$ for 24 hours. 8 to 10 colonies were picked to perform a Miniprep DNA plasmid extraction.

\subsubsection{Plasmid extraction}

For mini preparation colonies were transferred to $5 \mathrm{ml}$ of Luria-Bertani Medium (LB) and shaked at $200 \mathrm{rpm}$ for 16 hours at $37^{\circ} \mathrm{C}$ and then $1 \mathrm{ml}$ of the bacteria suspension was used for plasmid extraction. To extract the plasmid DNA, the PeqGold Plasmid Miniprep Kit (Table 6) was used as recommended by the manufacturer.

For midi preparation $1 \mathrm{ml}$ of a small scale culture (see above) was transferred into $50 \mathrm{ml}$ of LB and shaked at $200 \mathrm{rpm}$ overnight at $37^{\circ} \mathrm{C}$. For extraction of the plasmid DNA the Nucleobond AX100 kit (Table 6) was used according to the manufacturer's instructions (Macherey-Nagel, Düren, Germany). 


\subsubsection{Restriction of amplificates and recombinant plasmids and sequencing}

For restriction digests, enzymes by Fermentas were used (Table 6). To verify insertion of PCR amplification products into pCRII constructs a restriction analysis using EcoRI. EcoRI sites flank the TA cloning site of pCRII. EcoRI was used for inserts without a EcoRI site. A reaction mix for Eco RI restriction was prepared using 300-500ng of DNA, and 10 Units Eco RI, in a total volume $10 \mu$ l containing 1x Buffer $\mathrm{H}(1 \mu \mathrm{l})$ completed with distilled water. Restriction was performed for $2 \mathrm{~h}$ at $37^{\circ} \mathrm{C}$ followed by an electrophoresis using $1 \%$ agarose and $5 \mu \mathrm{l}$ Ethidium bromide to visualise the RVFV NSs band from the backbone vector pCRII.

Finally, positive plasmids were sequenced using the primers M13 forward (5'GTAAAACGACGGCCAG3') and M13 reverse (5'CAGGAAACAGCTATGAC3') to determine orientation and sequence integrity of the cloned NSs cDNAs as well as the presence of the C-terminal added FLAG tag (Seqlab Laboratories, Göttingen, Germany). To prepare the pI.18 for ligation, it was double digested using SalI and BgIII, using $3 \mu \mathrm{g}$ pI.18, 30 Units SalI, 30 Units BgIII, in a total volume of $60 \mu$ l containing 10xBuffer fast digest $(5 \mu \mathrm{l})$ completed with distilled water incubated $2 \mathrm{~h}$ at $37^{\circ} \mathrm{C}$.

To liberate the insert of the recombinant pCRII constructs for ligation into pI.18 a double digestion was performed using 250ng pCRII vector $1 \mathrm{Unit} / \mu \mathrm{l}$ SalI fast digest, $1 \mathrm{Unit} / \mu \mathrm{l}$ BglII fast digest, in a total volume of $50 \mu$ l containing 10x Buffer $(5 \mu \mathrm{l})$ completed with distilled water incubate $2 \mathrm{~h}$ at $37^{\circ} \mathrm{C}$.

\subsubsection{Expression and detection of RVFV-NSs}

\subsubsection{Assessment of the IFN- $\beta$ promoter activation by RVFV-NSs proteins}

The inhibitory effect of RVFV-NSs on VSV-RNA-mediated IFN- $\beta$ promoter activation was tested in a dual luciferase reporter assay. $1 \times 10^{5}$ Vero E6 cells were cotransfected with p125-luc (firefly luciferase cDNA expression driven by the human IFN- $\beta$ promoter), pRL-SV40 (renilla luciferase cDNA expression driven by the $\mathrm{SV} 40_{\text {early }}$ promoter, Promega, Mannheim, Germany) and pI.18-RVFV-NSs (NSs cDNA expression driven by the hCMV-IE promoter linked to the hCMV intron A in plasmid pI.18) using FuGene HD (Roche Biochemica, Mannheim, Germany) according to the manufacturer's instructions. After $24 \mathrm{~h}$ the transfected cells were either stimulated with RNA from VSV-infected Vero 
E6 cells or with RNA from uninfected Vero E6 cells as a control. RNA of VSV-infected cells contains high amounts of viral RNA with an unmodified triphosphate 5' end which is a potent inducer of IFN- $\beta$ expression [86]. For this purpose, cells were transfected with $1 \mu \mathrm{g}$ RNA using FuGene HD. Cells were lysed $16 \mathrm{~h}$ post stimulation and a Dualluciferase assay performed according to the manufacturer's instructions (Table 6) using a Fluostar Optima reader (Table 11).

During this step, the cotransfection using two constructs: one with the NSs-RVFv and the other with the Firefly luciferase is done into sterile TC-Plate 12 well using Vero E6 cells cultured as described in Chapter 2.2.4.3.

A master mix was prepared with pRL SV40 $(0.74 \mu \mathrm{g} / \mu \mathrm{l})$ and p125 Luc $(0.5 \mu \mathrm{g} / \mu \mathrm{l})$, and OptiMEM, then added to each purified RVFv-NSs to be tested for the Firefly luciferase and Renilla luciferase activities with FuGene. Control purified NSs of known virulent RVFV Z1 and attenuated clone 13 were also tested in the same plaque. After 24 hours incubation at $37^{\circ} \mathrm{C}$, the transfected Vero E cells were stimulated using $1 \mu \mathrm{g}$ Vero cell RNA and VSV RNA. After 16 hours incubation period at $37^{\circ} \mathrm{C}$, the cultured, transfected, and stimulated cells were submitted to a passive lysis using the Dual-luciferase Reporter Assay System (Table 6) and then the FF-Luc and Ren-Luc activities were respectively measured using the FLUOstar Optima reader (Table 11).

\subsubsection{Monitoring NSs filament formation in the nucleus of infected Vero E6 cells by indirect immunofluorescence assay}

Vero E6 cell were cultured on coverslips and transfected with RVFV-NSs Flag expression plasmids using FuGene HD (Roche) as transfection reagent. At 24 hours post transfection, the cells were fixed with $3 \%$ paraformaldehyde and permeabilized with $0.5 \%$ TritonX100 (Sigma, Deisenhofen, Germany). The permeabilized cells were then incubated with a monoclonal mouse anti-FLAG antibody (Sigma) as primary antibody (diluted 1:200 in PBS containing 1\% FCS), followed by incubation with an Alexa Fluor 555 labelled anti-mouse IgG (Invitrogen) as secondary antibody. Then coverslips were mounted in Fluosave mounting medium (Calbiochem, Bad Soden, Germany). After solidification of the mounting medium, the slides were examined by fluorescence microscopy using a Nikon TE2000-S inverted microscope (Table 11). 


\subsubsection{Manipulation of cells}

\subsubsection{Preparation and infection of plasmacytoid dendritic cells}

Buffy coats of healthy donors were kindly provided by the NSTOB Blood Bank (Springe, Germany), Deutsches Rotes Kreuz (Table 4). The pDCs were generated using the Diamond Plasmocytoid Dendritic Cell Isolation Kit human (Table 6) according to the manufacturer's instructions. The PBMC from anticoagulated blood or buffy coat PBMCs were purified by Ficoll gradients (Pharmacia) and purified by negative selection, which retains the non-plasmacytoid cells on a column and collects the pDCs by magnetic separation. This step was followed by another step of positive selection retaining the pDCs on a second column from which the pDCs were finally collected after some washing steps. The purity of the cell cultures was approximately $96 \%$, as determined by flow-cytometry analysis monitoring expression of CD123high and CD14low using CD123-fluorescein isothiocyanate (FITC) and CD14-phycoerythrin (PE) for staining.

For this purpose the pDCs were resuspended $5 \times 10^{5}$ cells per ml in 90\% RPMI 1640 medium, 10\% FCS, $2 \mathrm{mM}$ glutamine, $100 \mathrm{IU}$ penicillin/ml and $100 \mathrm{mg}$ streptomycin/ml for 12hours in the presence of IL-3 (BD Pharmingen). One part of the cell suspension was sampled for FACS analysis staining, to check the purity of the pDCs. Twenty $\mu 1$ of pDCs suspension were incubated for 15 minutes with CD123, CD303 antibodies (BD Pharmingen), the same volume was used for the isotype control using $\mathrm{ABC}$ mouse $\mathrm{IgG1}$, PRCP/CY55 mouse IgG (BD Pharmingen) to check for unspecific binding of the antibodies. The cells were then seeded into twelve well plates with a concentration of $2 \times 10^{5}$ cells per well. After overnight incubation at $37^{\circ} \mathrm{C}$ and $5 \% \mathrm{CO}_{2}$, the pDCs were used for infection experiments. During infections experiments a multiplicity of infection (MOI of 10) was used. Supernatants were collected at 12 hours, 24 hours, 48 hours, and 72 hours post infection and the harvested cells were tested for surface marker expression by FACS analysis using a cytokine bead array (Table 6).

\subsubsection{Preparation and infection of myeloid dendritic cells}

The mDCs were generated using the Myeloid Dendritic Cell Isolation Kit Human (Table 6) according to the manufacturer's instructions. They were prepared from peripheral blood mononuclear cells (PBMCs) of healthy individuals as described by [105]. Buffy coat of healthy donors was kindly provided by the NSTOB Blood Bank (Springe, 
Germany), Deutsches Rotes Kreuz (Table 4). The PBMC from anticoagulated blood or buffy coat were purified by Ficoll gradients (Pharmacia). The non mDC fraction was depleted through an indirect magnetic label using a cocktail of biotin-conjugated monoclonal antibodies as primary labelling reagent, and anti-biotin-monoclonal antibodies conjugated to microbeads as secondary labelling reagent as described by the manufacturer. The adherent-cell fraction was further purified by using anti-CD2 and antiCD19 immunomagnetic beads (Dynal). The purity of the cell cultures was approximately $95 \%$, as determined by flow-cytometry analysis showing expression of CD1 $\mathrm{c}^{\text {high }}$ and $\mathrm{CD} 14^{\text {low }}$ using CD1c-fluorescein isothiocyanate (FITC) and CD14-phycoerythrin (PE) for staining.

Resultant iDCs which are the purified precursor mDCs were resuspended as $5 \times 10^{5}$ cells per $\mathrm{ml}$ in 90\% RPMI 1640 medium, 10\% FCS, $2 \mathrm{mM}$ glutamine, $100 \mathrm{IU}$ penicillin/ml and $100 \mathrm{mg}$ streptomycin/ml for 12 hours in the presence of $50 \mathrm{ng}$ granulocyte-macrophage colony-stimulating factor per $\mathrm{ml}$ (Leukomax; Novartis Pharma) and $500 \mathrm{U}$ IL-4 (Cellgenix) (Kindly provided by the Dept. of Virology, University of Freiburg).

\subsubsection{Vero E6 culture and virus stock generation}

Vero E6 cells were maintained in Dulbecco's modified Eagle's medium (DMEM) (C.C.pro GmbH Neustadt, Germany) plus 10\% fetal calf serum (FCS) (Biochrom AG, Berlin, Germany) supplemented with $100 \mathrm{IU}$ penicillin/ml, $100 \mathrm{mg} / \mathrm{ml}$ streptomycin and $20 \mathrm{mM}$ L-Glutamine.

For virus-stock generation, Vero E6 cells were grown in cell-culture flasks until they reached $80 \%$ confluence. The growth medium was removed and the cells were inoculated with 0,01 MOI wtRVFV ZH548 or the RVFV clone 13 in $5 \mathrm{ml}$ infection medium (DMEM, 2\% FCS, $20 \mathrm{mM}$ HEPES). After incubation for $1 \mathrm{~h}$ at $37^{\circ} \mathrm{C}$, the virus inoculum was removed and replaced by regular growth medium. At $72 \mathrm{~h}$ postinfection, the virus supernatants were harvested and cell debris was removed by centrifugation (3000 $g$ for 5 min at $4{ }^{\circ} \mathrm{C}$ ). Virus stocks were stored at minus $80^{\circ} \mathrm{C}$ and thawed immediately before use.

\subsubsection{RVFV infections of Vero E6 cells in the presence of IL-6}

After culture for 24 hours, the subconfluent Vero E6 cells were serum-deprived for one day and treated with human IL-6 (R\& D System Inc., Minneapolis, USA) using 20ng/ml as described previously by [106-107]. On day three after plating out, the Vero E6 cells 
were infected with RVFV wtZH548 at a MOI of 10. The culture medium was refreshed by adding IL-6 $20 \mathrm{ng} / \mathrm{ml}$ on each day after infection until the third day after infection, on which results were read.

\subsubsection{RVFV infection of DCs}

DC infections were performed directly in the wells where the cells were generated. Ultra violet light (UV) inactivated wtRVFV ZH548, the naturally avirulent RVFV strain clone 13 and the UV inactivated clone 13 were diluted in serum free RPMI 1640 (C.C.pro GmbH Neustadt, Germany) and added to the cells at a multiplicity of infection of 10. A mock control consisting of the supernatant of non-infected Vero E6 was also used and added to the DC culture.

\subsubsection{Flow cytometry analysis}

pDCs infected, mock infected or incubated with UV-inactivated wtRVFV ZH548 and UV-inactivated clone 13, were collected at 12 hours, 24 hours, 48 hours, and 72 hours after infection, washed in PBS and incubated with antibodies against CD80, CD86, CD83, CD54, CD58, CD40, CD95, MHCI and MHCII surface molecule markers (Table 7). These antibodies are labelled with chromogens and allow differentiation of each surface marker by a specific colour. The following chromogens were used: Allophycocyanin (APC) conjugated to anti CD40, fluorescein isothiocyanate (FITC) conjugated to $\mathrm{CD} 58$, phycoerythrin (PE) conjugated to $\mathrm{CD} 54$, pacific blue conjugated to major histocompatibility I (MHCI), and Pecy7 conjugated to CD11c in one set.

In another set FITC was conjugated to CD80, phycoerythrin (PE) to CD83, APC to CD86, pacific blue (Pac Blue) to MHCII and Pecy7 to CD11c. The isotype control was prepared in a separate set with a mix of PE, FITC, APC, PE-Cy7, and PerCP-Cy5.5 antibodies.

The same procedure was performed after $\mathrm{mDC}$ infection. The anti-CD123-FITC was replaced by anti-CD1c-FITC. After staining the samples were fixed with 3,7 \% paraformaldehyde for 15 min and then analysed on a FACS Canto II (Becton Dickinson). Raw data was subsequently processed using the Flow Jo software (Table 13). 


\subsubsection{Cytokine detection}

Plasmacytoid dendritic cells (pDCs) and myeloid dendritic cells (mDCs) were isolated from human buffy coat and infected with Rift Valley Fever virus strains.

\subsubsection{Monitoring proinflammatory cytokine production in DC supernatants after RVFV infection}

To assess proinflammatory cytokines, the pDCs were resuspended as $5 \times 10^{5}$ cells per ml in 90\% RPMI 1640 medium, 10\% FCS, 2 mM glutamine, 100 IU penicillin/ml and 100 $\mathrm{mg}$ streptomycin/ml for 12 hours in the presence of IL-3 (BD Pharmingen). The cells were then seeded in twelve well plates with a concentration of $2 \times 10^{5}$ cells per well. After overnight incubation at $37^{\circ} \mathrm{C}$ in $5 \% \mathrm{CO}_{2}$ environment, the DCs were infected at a multiplicity of infection (MOI) of 10. After 12 hours, 24 hours, 48 hours, and 72 hours post infection supernatants were collected and kept at $-80^{\circ} \mathrm{C}$ until further use. The presence of the interleukins IL-6, IL-8, IL-10, IL-1ß, IL-12p70 and TNF $\alpha$ was assessed by Cytometric Bead Array (CBA) using the Human Inflammatory Cytokines Kit (Table 6). The assays were performed according to the manufacturers protocol. Raw data was subsequently processed using the FCAP software (Table 13).

\subsubsection{Monitoring interferon- $\alpha$ production in DC supernatants after RVFV infection}

Plasmacytoid dendritic cells (pDCs) and myeloid dendritic cells were isolated from human buffy coat and incubated with different RVFV strains. Both DC subsets were analyzed for interferon- $\alpha$ production. Supernatants were harvested at $72 \mathrm{~h}$ p.i from pDCs and mDCs infected with replication competent wtRVFV ZH548, the naturally avirulent strain clone 13, and the corresponding UV-inactivated srains, and supernatant of non infected Vero E6 cell as control using the Human IFN- $\alpha$ Kit (PBL InterferonSource, Piscataway USA (Table 6) according to the manufacturer's protocol.

\subsubsection{Tissue Culture Infectious Dose 50 (TCID50) assays}

Viral stocks or supernatant from infected pDCs or mDCs were titrated on Vero E6 cells supplemented with $10 \% \mathrm{FCS}$, and $100 \mathrm{IU} / \mathrm{ml}$ of penicillin, and $100 \mu \mathrm{g} / \mathrm{ml}$ of streptomycin. Viral stocks were serially 10-fold diluted in free serum DMEM. $100 \mu 1$ of each dilution were inoculated on VeroE6 cell monolayers in a 96 flat bottom well plate. 
After $72 \mathrm{~h}$ p.i. incubation at $37^{\circ} \mathrm{C}$ in an environment of $5 \% \mathrm{CO}_{2}$, the result was read by eye under an inverted microscope for the presence or not of CPE in the wells. 


\section{$3 \quad$ Results}

\subsection{Cloning of NSs of RVFV isolates into the eukaryotic expression vector pI.18}

In order to assay the natural activity of the NSs proteins of 26 RVFV strains, the NSs cDNAs were ligated into the expression vector pI.18. First, NSs cDNAs were obtained by reverse transcription of viral RNA followed by PCR amplification and ligation into the TA-vector pCRII. Subsequently, the cDNAs were subcloned into the eukaryotic expression vector pI.18 (chapter 2.2.2.4).

An example of the successful cloning procedure is shown for RVFV-NSsR17 in Figure 9. The expected band size for RVFV-NSs after PCR amplification is around 800 base pairs, which indeed was observed for R17-NSs (Figure 9A). Ligation of the NSs cDNA into pCRII was monitored by restriction with EcoRI, which resulted in a $4000 \mathrm{bp}$ band (vector backbone), a $600 \mathrm{bp}$ band (3' fragment of NSs cDNA) and a $200 \mathrm{bp}$ band (5' fragment of NSs cDNA) (Figure 9B). After subcloning the NSs cDNA into the eukaryotic expression vector pI.18, successful ligation was confirmed by BglII/SalI restriction, which resulted in a 4300 bp band (vector backbone) and a 800 bp band (NSs cDNA) (Figure 9C).

From the 33 RVFV isolates available, 26 RVFV NSs cDNAs were successfully ligated into pI.18. For the remaining isolates it was not possible to amplify the NSs cDNA most probably due to minor quality of the RNA used as template for reverse transcription.

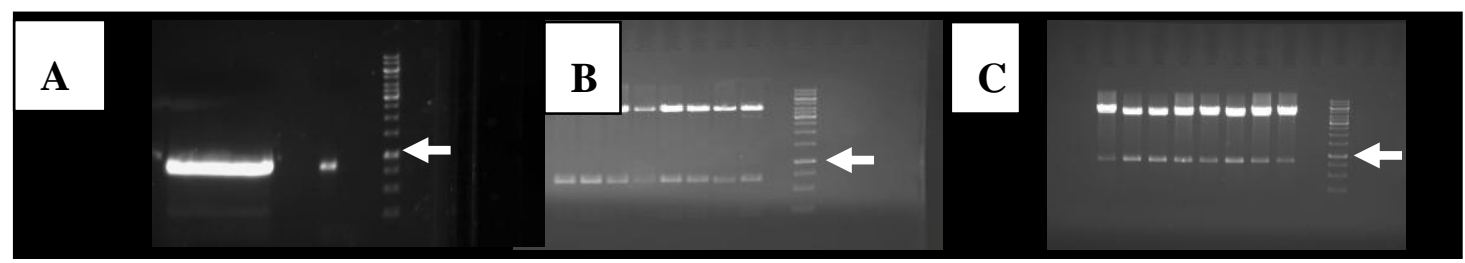

Figure 9. (A) PCR product amplified from cDNA of RVFV-NSs R17 in 0.8 percent agarose gel, (B) eight clones of the pCRII-RVFV-NSs R17 construct after restriction using EcoRI in 1 percent agarose gel. The $200 \mathrm{bp}$ fragment of the 5'NSs fragment is hardly visible, (C) eight clones of the pI.18RVFV-NSs R17 construct after restriction using SalI and BglII in 1 percent agarose gel. The arrows indicate the $1000 \mathrm{bp}$ band of the DNA marker. 


\subsection{Inhibition of IFN- $\beta$ promoter activation by RVFV-NSs}

To test whether expression of the NSs cDNAs inhibits the IFN- $\beta$ promoter activation, Vero E6 cells were cotransfected with recombinant expression plasmids (pI.18) for (i) NSs of wild type virus RVFV ZH548 as a positive control, (ii) NSs of the attenuated RVFV clone 13 as a negative control, and (iii) NSs of each of the 26 RVFV isolates together with the reporter plasmids p125-luc and pRL-SV40. After stimulation with RNA of VSV-infected Vero E6 cells or uninfected cells IFN- $\beta$ promoter activities were determined by luciferase assay (chapter 2.2.3.1).

The results of the luciferase assays (Figure 10) show that two RVFV-NSs clones (R7 and R18) failed to suppress IFN- $\beta$ promoter activation after stimulation with RNA of VSVinfected cells as indicated by the high firefly luciferase activities when compared to wtRVFV ZH548- NSs (reference virulent wild-type strain). In contrast, the other RVFVNSs clones showed efficient suppression of IFN- $\beta$ promoter activity since only low firefly luciferase activities were detected.

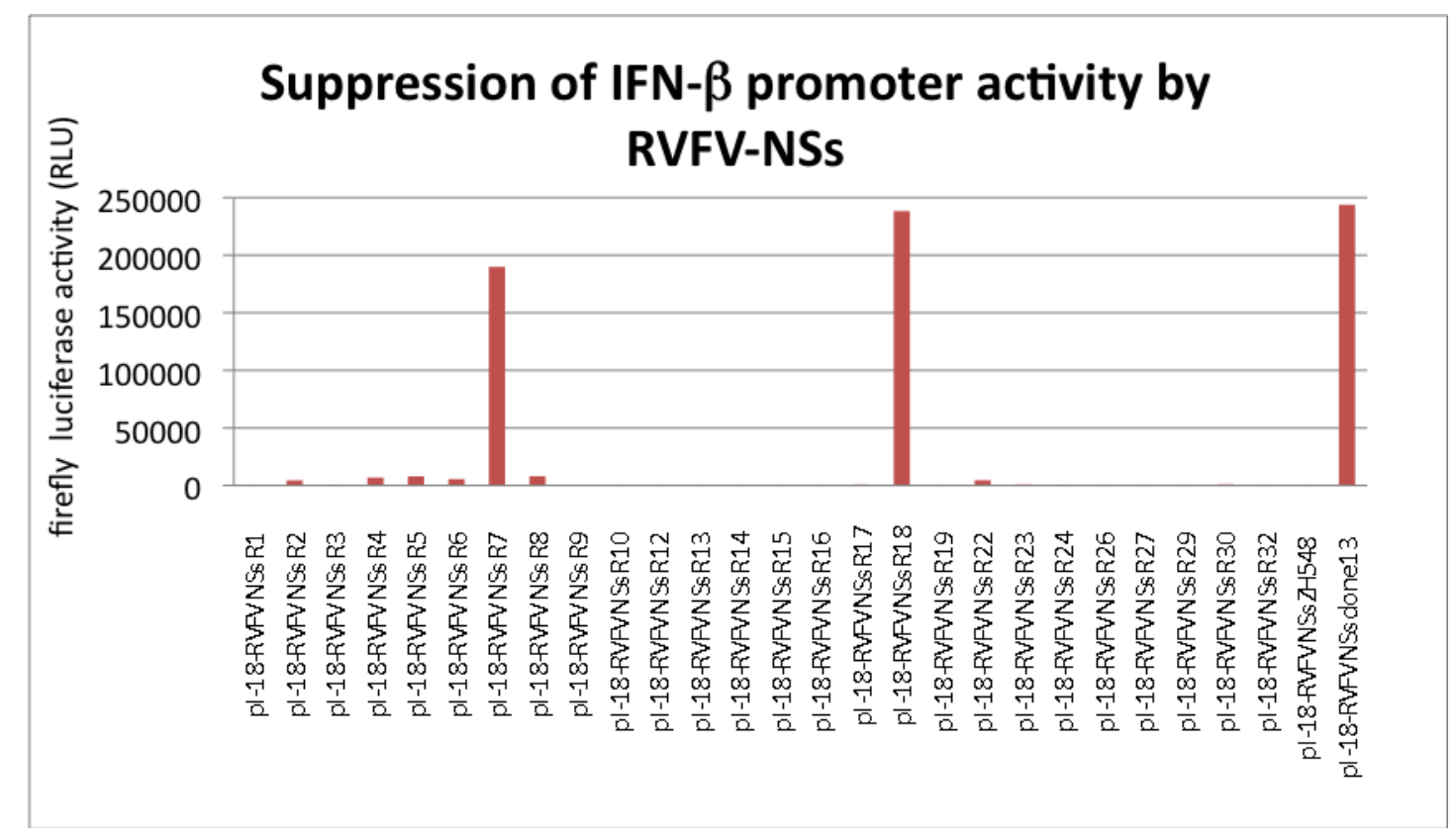

Figure 10. Effect of NSs on IFN- $\beta$ promoter activity after stimulation with RNA from VSV-infected cells for the 26 RVFV-NSs clones, RVFV-NSs ZH548, and RVFV-NSs clone13 in a luciferase reporter assay. Relative light units (RLU) represent the readout for firefly luciferase activity, which indicates the activity of the IFN- $\beta$ promoter. The diagram represents the mean values of five independent experiments for each NSs clone. 


\subsubsection{Comparison of the IFN- $\beta$ promoter suppressing activity of the RVFV-NSs} clones with the reference wild-type ZH548 RVFV-NSs

The results show significant differences $(\mathrm{p} \leq 0.05)$ in the ability to inhibit IFN- $\beta$ promoter activation between the wild type RVFV ZH548-NSs and R2, R4, R5, R6, R7, R18, R23, R26, R27, R29, R30 RVFV-NSs (Figure 11). Although significant, the observed differences are small with the marked exception of NSs-R7 and NSs-R18. There is no significant difference in blocking IFN- $\beta$ promoter activation between the wtRVFV ZH548-NSs and the remaining RVFV-NSs clones.

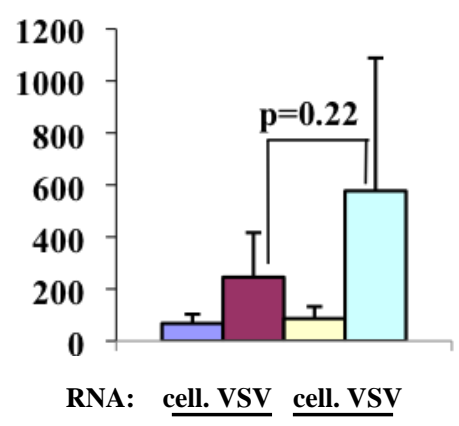

NSs: R1 ZH548

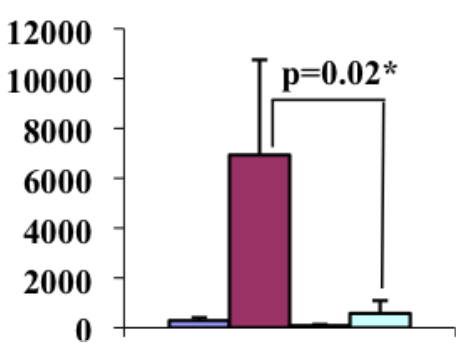

RNA: cell. VSV cell. VSV

NSs: R4 ZH548

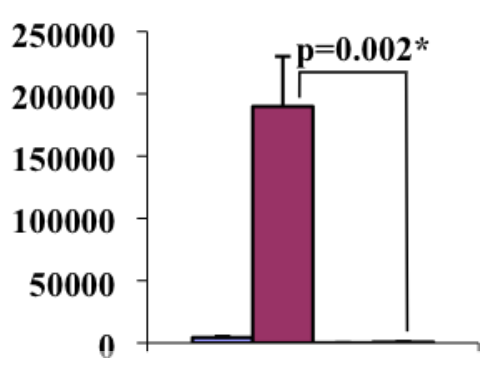

RNA: cell. VSV cell. VSV

NSs: $\quad$ R7 ZH548

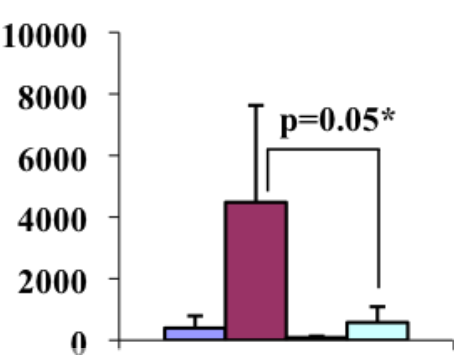

RNA: cell. VSV cell. VSV

NSs: R2 ZH548

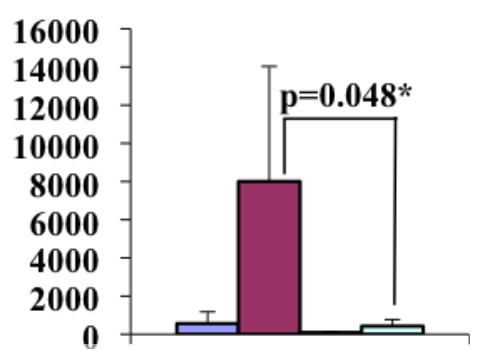

RNA: cell. VSV cell. VSV

NSs: R5 ZH548

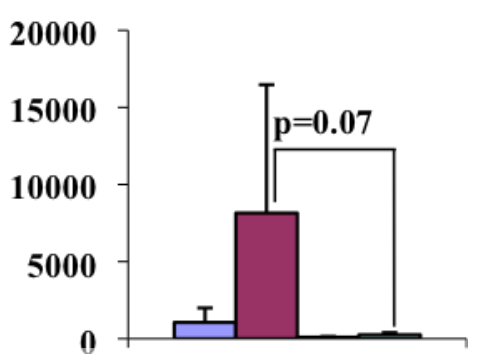

RNA: cell. VSV cell. VSV

NSs: R8 ZH548

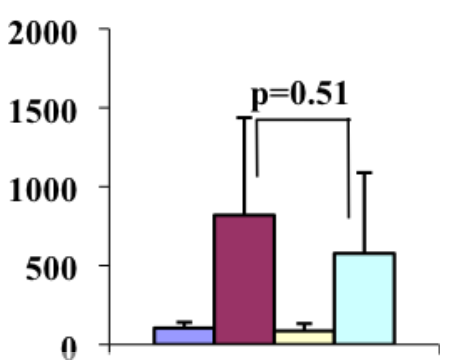

RNA: cell. VSV cell. VSV

NSs: R3 ZH548

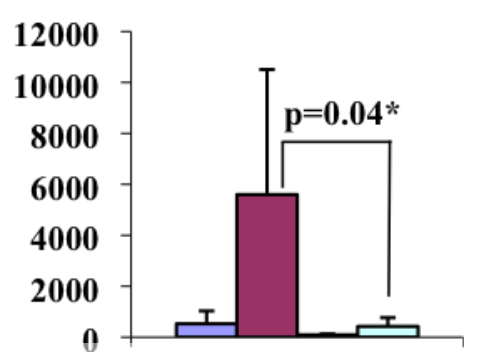

RNA: cell. VSV cell. VSV

NSs: R6 ZH548

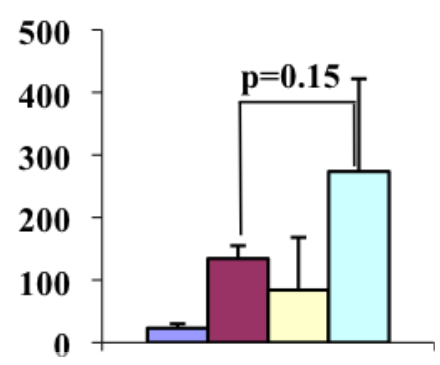

RNA: cell. VSV cell. VSV

NSs: R9 ZH548 


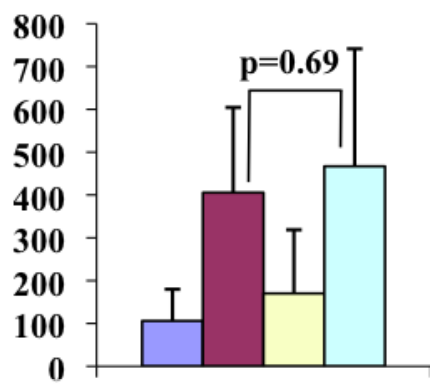

RNA: cell. VSV cell. VSV

NSs: R10 ZH548

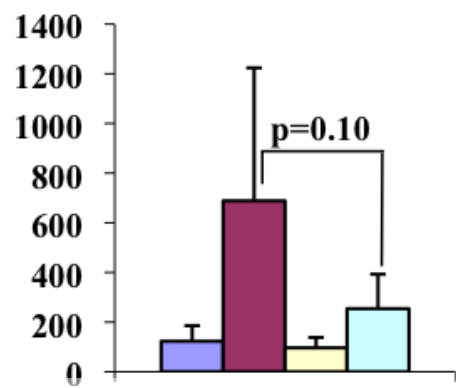

RNA: cell. VSV cell. VSV

NSs: R14 ZH548

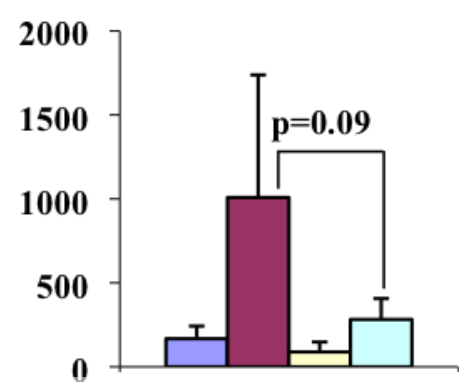

RNA: cell. VSV cell. VSV

NSs: R17 ZH548

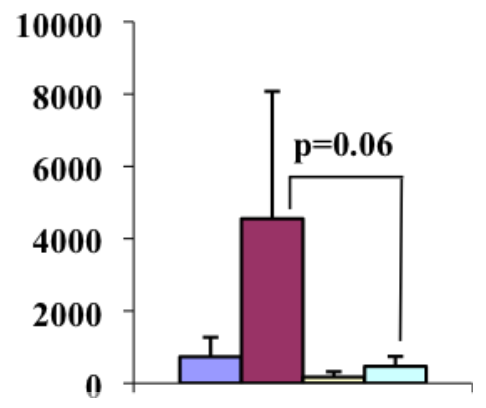

RNA: cell. VSV cell. VSV

NSs: R22 ZH548

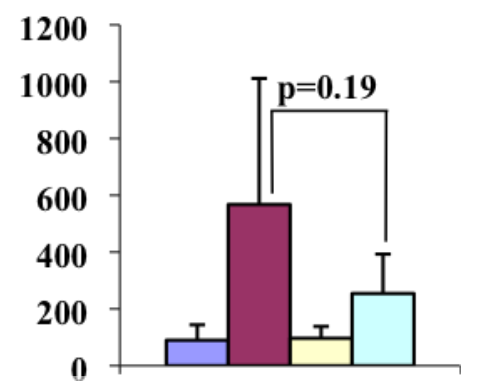

RNA: cell. VSV cell. VSV

NSs: R12 ZH548

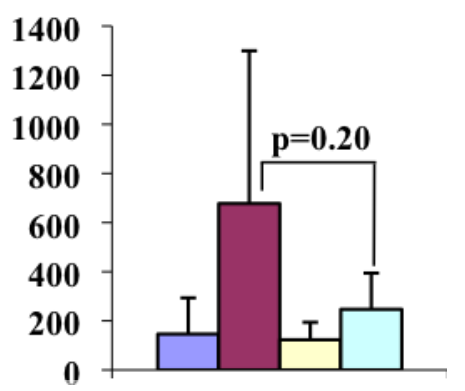

RNA: cell. VSV cell. VSV

NSs: R15 ZH548

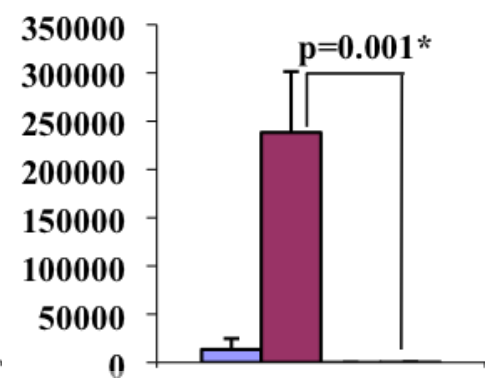

RNA: cell. VSV cell. VSV

NSs: R18 ZH548

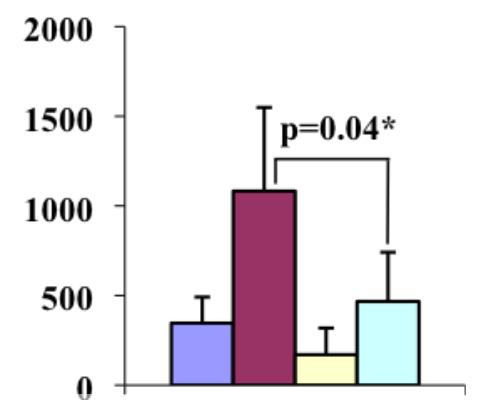

RNA: cell. VSV cell. VSV

NSs: R23 ZH548

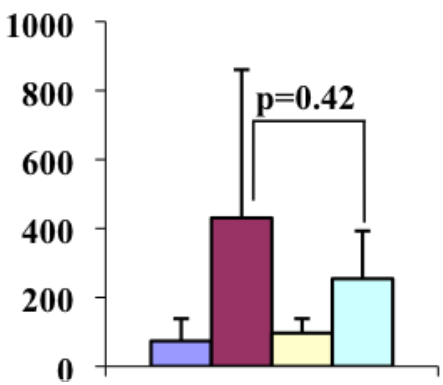

RNA: cell. VSV cell. VSV

NSs: R13 ZH548

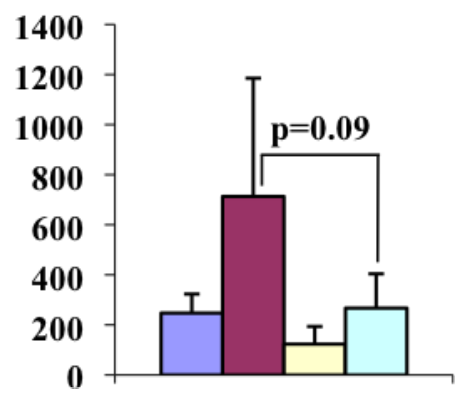

RNA: cell. VSV cell. VSV

NSs: R16 ZH548

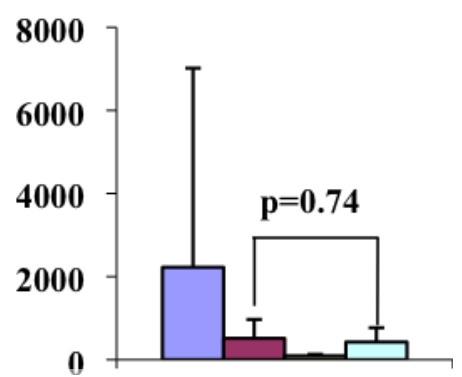

RNA: cell. VSV cell. VSV

NSs: R19 ZH548

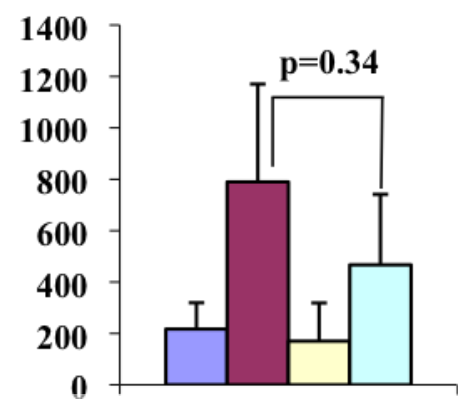

RNA: cell. VSV cell. VSV

NSs: R24 ZH548 

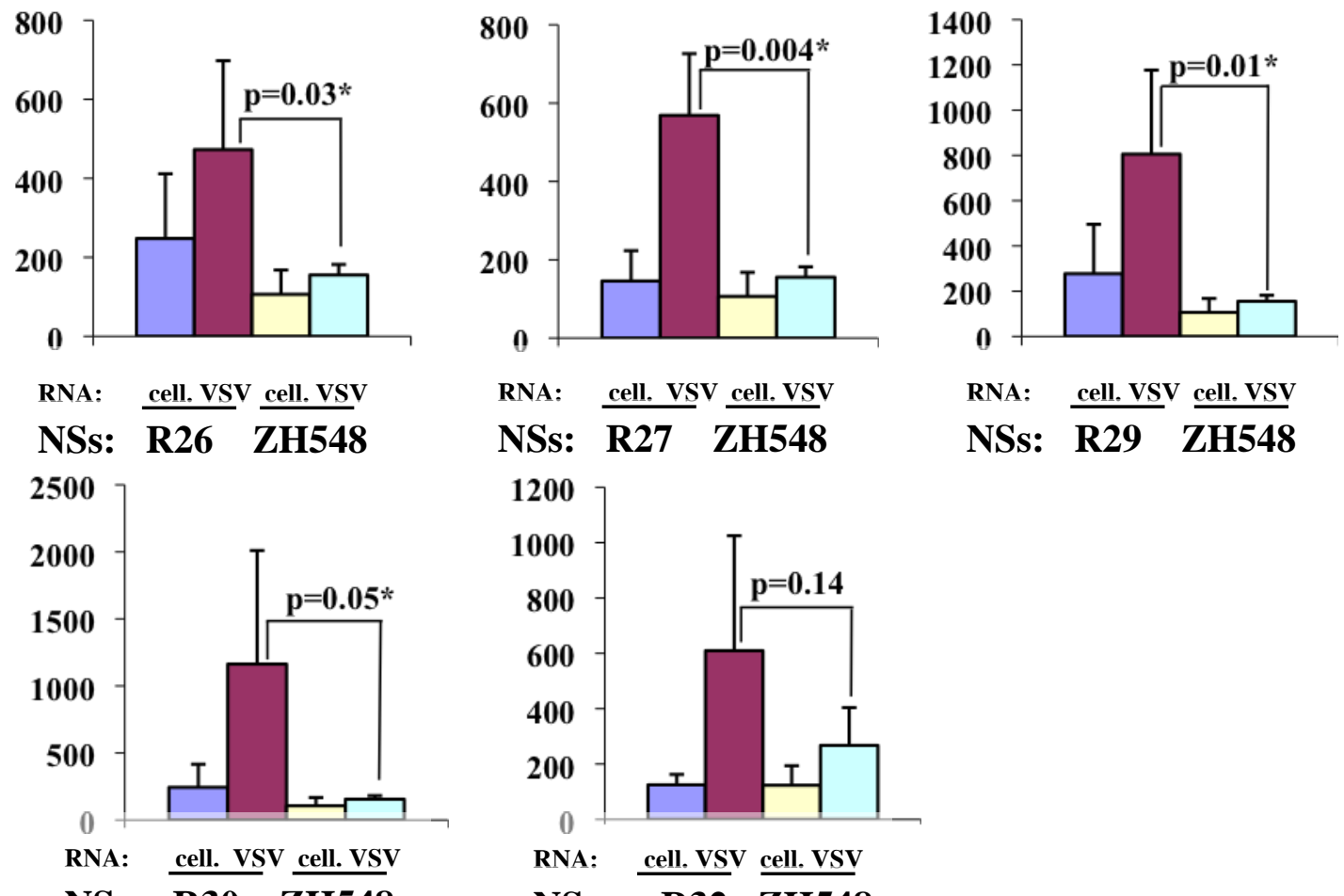

RNA: cell. VSV cell. VSV

NSs: R29 ZH548

NSs: R30 ZH548

NSs: $\quad$ R32 ZH548

Figure 11. IFN- $\beta$ promoter activity measured by firefly luciferase activity after stimulation with RNA of VSV-infected Vero cells (VSV) or with RNA of uninfected cells (cell.) for RVFV-NSs R1-10, R12-19, R22-24, R26-30 and R32 in comparison to wt RVFV-NSs ZH548. The diagrams represent the mean values of five independent experiments for each NSs clone; $p$ values were calculated by Student's t-test. Significant differences $(\mathrm{p}<=0.05)$ are marked with an asterisk. NSs-R7 and NSs-R18 show a significant loss of IFN- $\beta$ promoter inhibition.

\subsection{Comparison of filament formation in the nucleus of Vero cells transfected with 26 RVFV-NSs expression plasmids}

In order to find out if the NSs activity observed could be correlated to filament formation in the nucleus of Vero E6 cell immunofluorescence staining was performed. It allowed detection of filamentous structures in the nucleus of Vero E6 cells transiently transfected with FLAG-tagged RVFV-NSs proteins for most RVFV strains and the wild-type strain ZH548-NSs (Figure 12). However for the NSs of three RVFV isolates (R7, R10, and R18) nuclear filament formation was not observed. 


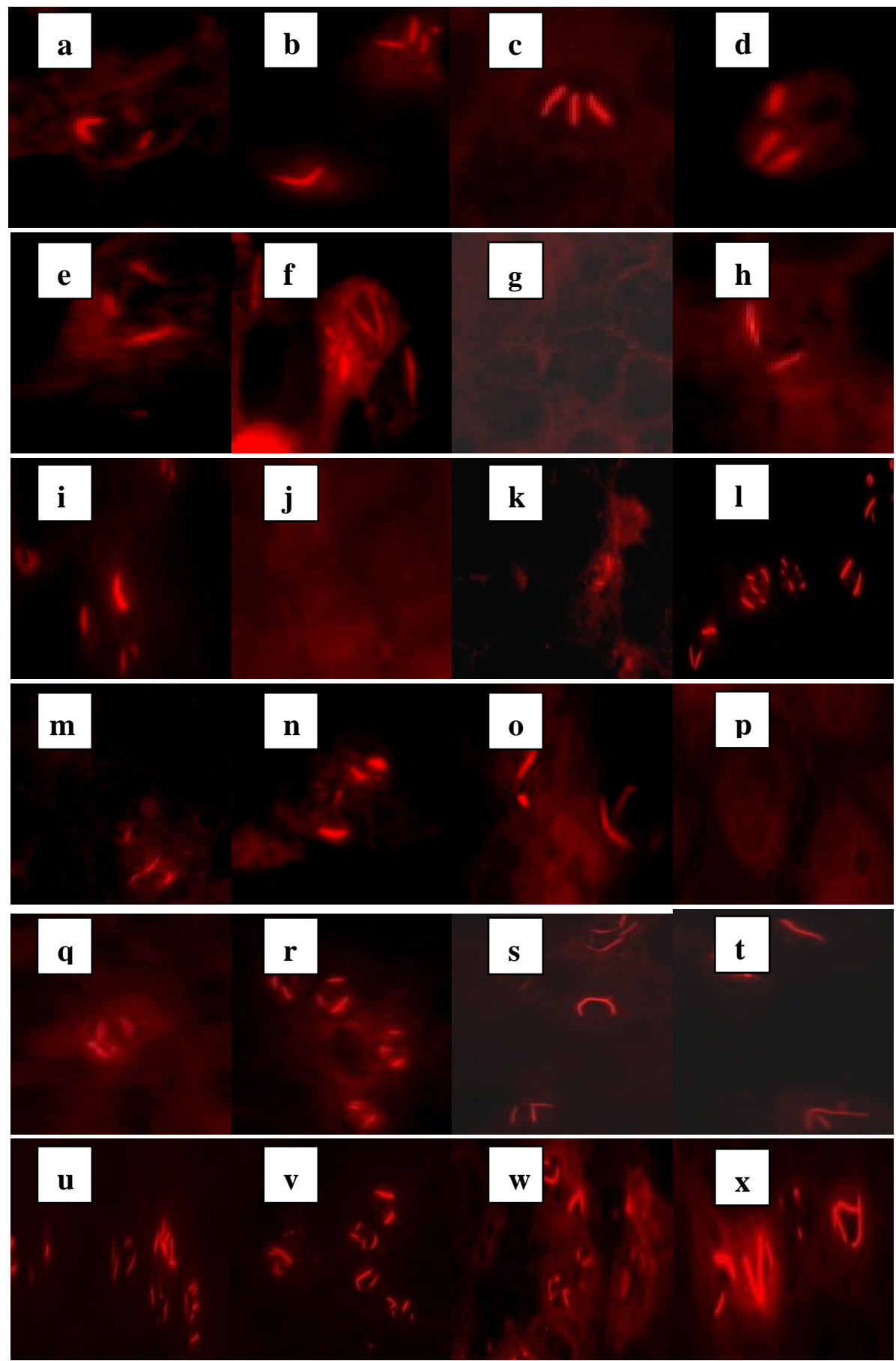




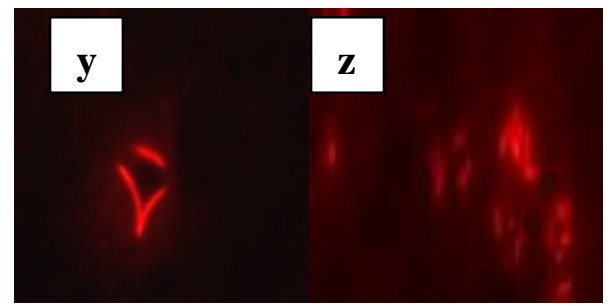

Figure 12. Filamentous structure formation of RVFV-NSs in transfected cells. (a) R1, (b) R2, (c) R3, (d) R4, (e) R5, (f) R6, (g) R7, (h) R8, (i) R9, (j) R10, (k) R12, (l) R14, (m) R15, (n) R16, (o) R17, (p) R18, (q) R19, (r) R22, (s) R23, (t) R24, (u) R6, (v) R27, (w) R29, (x) R30, (y) R32, (z) ZH548. No filament formation was observed for R7 (g), R10 (j) and R18 (p).

\subsection{A point mutation in the NSs sequence of RVFV-NSs R7 affects NSs function}

Sequencing of RVFV R18-NSs revealed a large in-frame deletion of 549 nucleotides starting at nucleotide position 46 of the NSs ORF, which is identical to the deletion observed in RVFV clone 13-NSs. The point mutation observed in R7-NSs and the inframe deletion observed in R18-NSs (which includes position 344 of the R7-NSs mutation) have similar effects: both NSs clones fail to inhibit IFN- $\beta$ promoter activation and do not form nuclear filaments.

All cloned NSs genes were sequenced and compared to the NSs sequence of the reference strain RVFV ZH548. Nucleotide exchanges were found in 71 positions of the NSs ORF, which has a length of $798 \mathrm{nt}$, however the vast majority of these mutations was silent. In the NSs sequence of RVFV isolates R1, R2, R3, R4, R5, R6, R7, R8 and R32 a G to A exchange at position 71 of the NSs ORF was detected which leads to the replacement of arginine by lysine on the amino acid level. With the exception of R7-NSs these NSs clones were efficient inhibitors of IFN- $\beta$ promoter activation (Figure 11) and formed nuclear filaments (Figure 12) indicating that this mutation has no effect on NSs function and stability. Frequent mutations were observed at positions 724-726. While the RVFV ZH548-NSs sequence contains the triplet ATT at these positions which corresponds to isoleucine, NSs clones R1 and R3 contain GTC (corresponding to valine), NSs of RVFV isolates R4, R5, R6, R7, R8, R22, R23, R24, R26, R27, R28, R29, R30 and R32 contain GTT (also corresponding to valine) and the NSs of the isolates R12, R13 and R14 contain ATC (which is a silent mutation). The remaining NSs sequences of isolates R9, R10, R15, R16 and R17 carry no mutations at these positions. Similar to the replacement of arginine by lysine in the N-terminal part of NSs, the isoleucin to valine exchange in the C-terminal part of NSs had no consequences with respect to suppression of IFN- $\beta$ promoter 
activation (Figure 11) and nuclear filament formation (Figure 12) R7-NSs contains an additional mutation at position 344 ( $\mathrm{T}$ to $\mathrm{C}$ ), which leads to the replacement of leucine by proline. The alignment of all NSs sequences can be found in chapter 7.6 in Appendix.

\subsection{A point mutation in the NSs sequence of RVFV-NSs R10 affects filament formation}

The sequence of RVFV NSs-R10 shows a point mutation in the open reading frame at the nucleotide position 320, which leads to the replacement of leucine by proline. Obviously this destroys the ability of NSs to form filaments (Figure 12j). However, the clone R10NSs was fully functional with respect to suppression of the IFN- $\beta$ promoter activation (Figure 10). This observation reveals that the promoter activation of IFN- $\beta$ and the nuclear filament formation clone do not correlate (Figure 12j). The observed phenotype is similar to the characteristics of NSs of RVFV strain MP12, which was derived from wtRVFV by serial tissue culture passages in the presence of 5-fluorouracil. Although this strain is attenuated in mice (probably due to mutations in all three genomic segments), MP12-NSs is an efficient inhibitor of IFN- $\beta$ promoter activation in cell culture. At the same time MP12-NSs has an impaired ability to form nuclear filaments which only occurs in the case of massive NSs overexpression [2]. Interestingly NSs-R10 is not identical to NSs-MP12, indicating that inhibition of IFN- $\beta$ promoter activation combined with a loss of filament formation is a phenotype, which also can be found in naturally occurring RVFV strains. The alignment of all NSs sequences can be found in chapter 7.6 in Appendix.

\subsection{RVFV replicates in $\mathrm{mDCs}$ but does not in pDCs}

The pDCs were generated from peripheral blood mononuclear cells (PBMC) using the 'Diamon Plasmocytoid Dendritic Cell Isolation Kit human' (Table 6) according to the manufacturer's instructions. The purity of the cell cultures was approximately $96 \%$, as determined by flow-cytometry analysis showing expression of CD123high and CD14low [CD123-fluorescein isothiocyanate (FITC), CD14-phycoerythrin (PE); BD Pharmingen] (see chapter 2.2.5.1). The mDCs were generated from peripheral blood mononuclear cells (PBMCs) of healthy individuals as described by [105] using the Myeloid Dendritic Cell Isolation Kit Human (Table 6) according to the manufacturer's instructions. The purity of the cell cultures was approximately $95 \%$, as determined by flow-cytometry analysis 
showing expression of CD1c high and CD14 low [CD1c-fluorescein isothiocyanate (FITC), CD14-phycoerythrin (PE); BD Pharmingen] (see chapter 2.2.5). The cells were then seeded in twelve well plates using 200.000 cells per well. After overnight incubation at $37^{\circ} \mathrm{C}$ in $5 \%$ carbon dioxide environment, the pDCs were infected at a multiplicity of infection (MOI) of 10 and then collected at $72 \mathrm{~h}$ following infection. In order to test the permissiveness of DCs to RVFV infection $\mathrm{TCID}_{50}$ tests were performed. To this aim, Vero E6 cells were seeded in a 96 well microplate using 100.000 cells per well and inoculated by serial 10-fold dilutions of wtRVFV ZH548, RVFV Clone13, their corresponding input virus stocks incubated at $37{ }^{\circ} \mathrm{C}$ for 72 hours as a readout for residual infectivity after $72 \mathrm{~h}$ (since virus inoculums were not removed from the non-adherent DCs), and mock control. The microplate tests were then kept at $37^{\circ} \mathrm{C}$ in an environment of $5 \% \mathrm{CO}_{2}$. The results were read 72 hours post infection under an inverted microscope to evaluate the presence of cythopathic effects. Wells were scored positive when a cythopathic effect (CPE) on the cell monolayer was observed and negative in the absence of a CPE. The results gave strong evidence that wtRVFV ZH548, like RVFV clone 13 do not replicate in pDCs contrary to their replication found in mDCs. Replication of RVFV in mDCs was more efficient when these cells were infected with wtRVFV ZH548 than when infected with clone 13.

A.

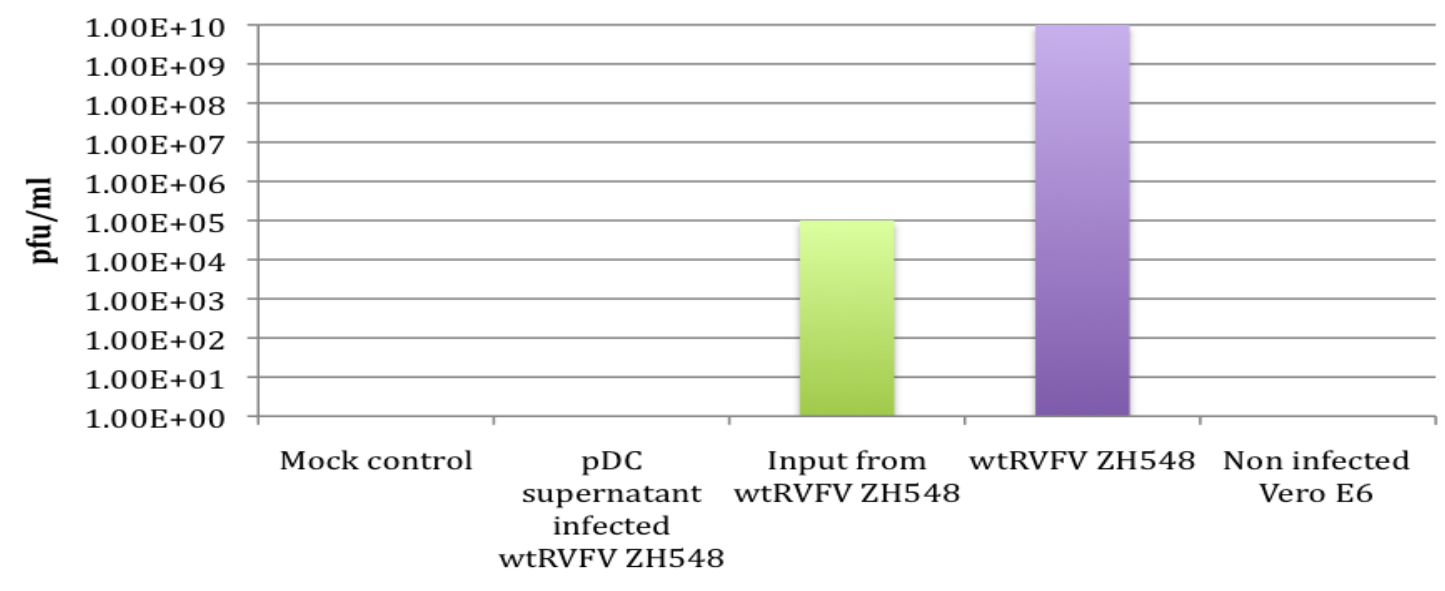


B.

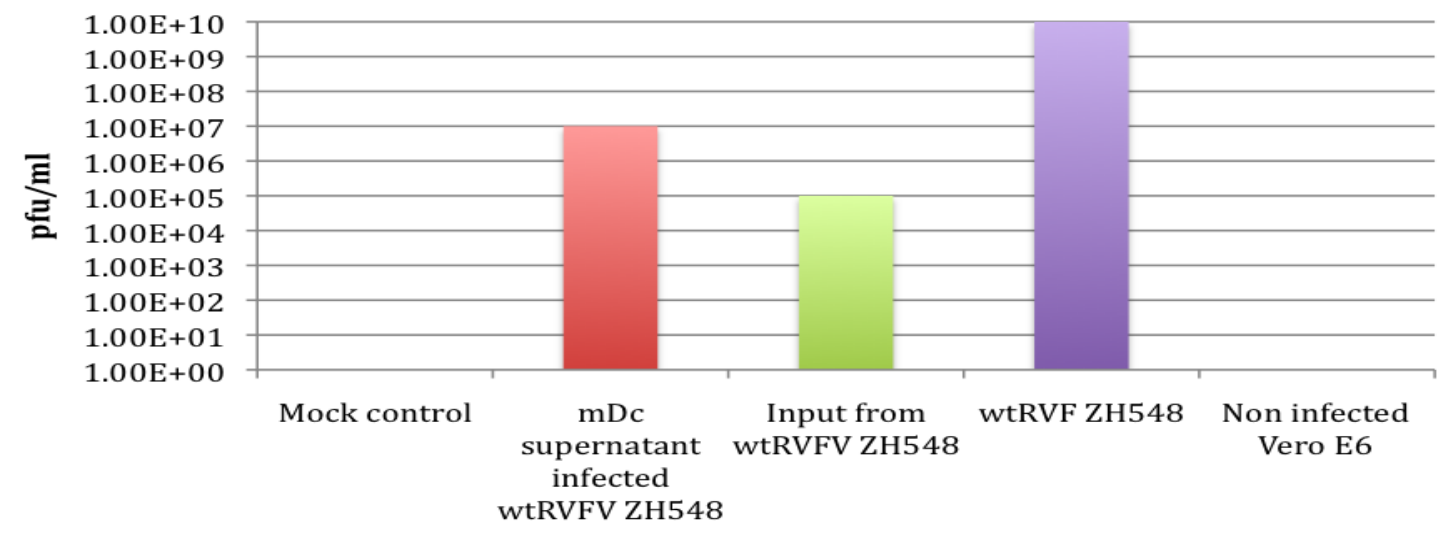

C.

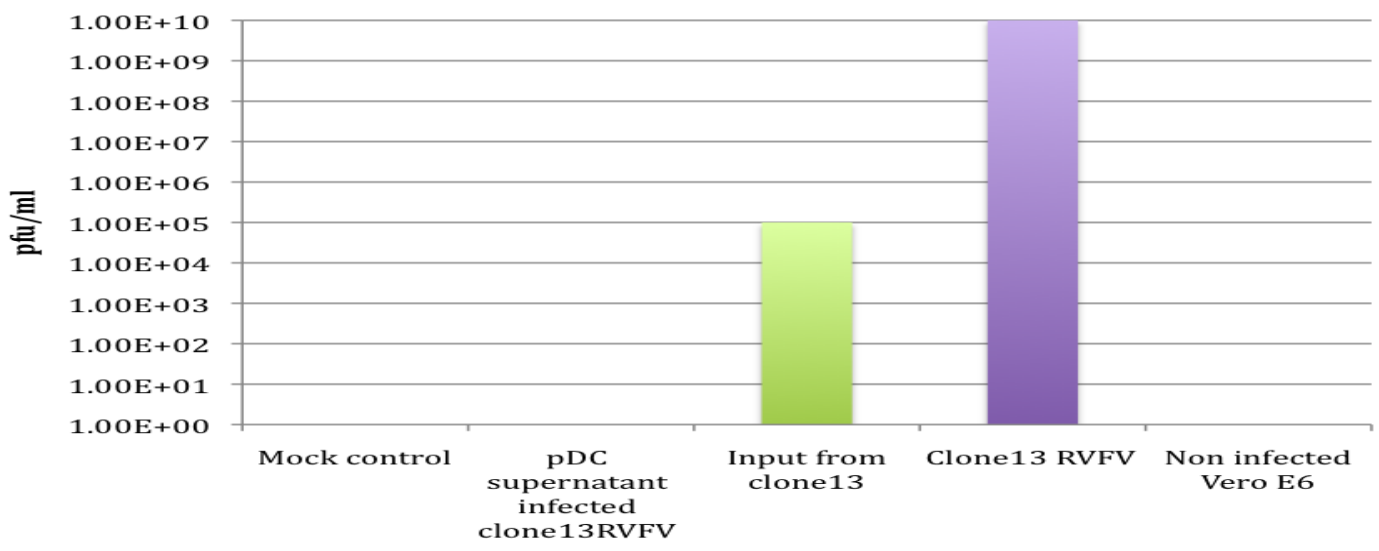


D.

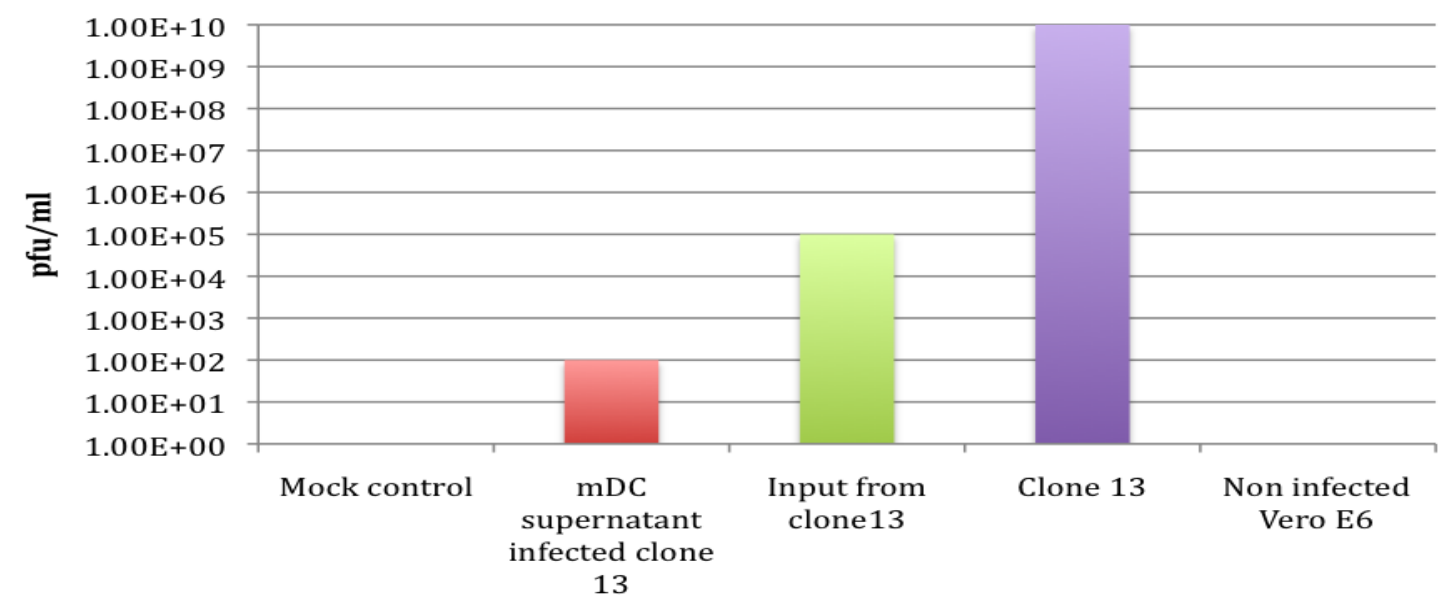

Figure 13. TCID 50 performed with VeroE6 cells as indicator cells. A and B, infection of DCs with wtRVFV ZH548; C and D, infection of DCs with RVFV clone 13. A: Absence of infectious virus was observed in supernatants collected from mock-infected pDCs and from pDCs infected with wtRVFV ZH548, and in supernatant of non-infected VeroE6 cells. Infectious virus could only be detected in input virus prepared from wtRVFV ZH548 (green bar) and in wtRVFV ZH548 virus stock (purple bar). B: Absence of infectious virus was observed in supernatant collected from mock-infected mDCs and from non-infected Vero E6 cells. Infectious virus was detected in supernatant collected from $\mathrm{mDC}$ infected with wtRVF ZH548 (red bar), in input virus prepared from wtRVF ZH548 (green bar) and in wtRVFV ZH548 virus stock (purple bar). C: Absence of infectious virus was observed in supernatant of mock-infected pDCs, in supernatant collected from pDCs infected with clone 13 and in supernatant of non-infected Vero E6 cells. Infectious virus was detected in input virus prepared from RVFV clone 13 (red bar) and in RVFV clone 13 virus stock (blue bar). D: Absence of infectious virus was observed in supernatants of mock-infected mDCs and in the supernatant of non-infected Vero E6 cells. Infectious virus was detected in supernatant collected from mDCs infected with wtRVFV ZH548 (yellow bar), in input virus prepared from RVFV clone 13 (red bar) and in RVFV clone 13 virus stock (blue bar).

\subsubsection{Proinflammatory cytokine production in mDCs infected by RVFV}

Cytokines modulate activation and maturation of DCs. In order to assess the cytokine production of RVFV infected mDC several pro inflammatory cytokines (IL-1 $\beta$, IL-6, IL8, IL-12p70, TNF $\alpha$, and IL-10) were analyzed by FACS analysis in mDCs infected with RVFV strains.

and Figure 23 to Figure 26 in the appendix show the expression of proinflammatory cytokines in mDCs infected with wtRFV ZH548 (red bar), with UV inactivated wtRVFV ZH548 (green bar), RVFV clone 13 (purple bar), UV inactivated RVFV clone 13 (blue bar), and mock control (dark blue bar) at 12h, 24h, 48h, and 72h (clockwise).

The results show that IL-1 $\beta$ production is very low and doesn't seem to show significant change during the time course of infection (Figure 23). At $72 \mathrm{~h}$ p.i. only the mock control 
and wtRVFV ZH548 produced levels of IL-1 $\beta$ which were above the background. Obviousely, mDCs don't produce IL-1 $\beta$ after infection with RVFV.

IL-6 levels were significantly increased after infection with RVFV ZH5458 and slightly increased after infection with RVFV clone 13. Interestingly, replication competent virus was not required for IL-6 induction since UV-inactivated virus preparation had a similar effect although less pronounced.

IL-8 was produced at all time points in infected and non-infected cells and the production dosen't seem to show significant change during the course of infection (Figure 15).

Our result denote a low amount of IL-10 and Tumor necrosis factor alpha (TNF $\alpha$ ) production in mDCs infected either by replication competent or UV inactivated wtRVFV ZH548 and RVFV clone 13 and the mock control. IL-10 and TNF $\alpha$ production were found at $12 \mathrm{~h}$ and $24 \mathrm{~h}$ p.i. but not at late time points such as 48 or $72 \mathrm{~h}$ p.i. (Figure 24 and Figure 25).

For interleukin 12p70 (IL-12p70), no production at all was observed even in the mock (Figure 26) control.
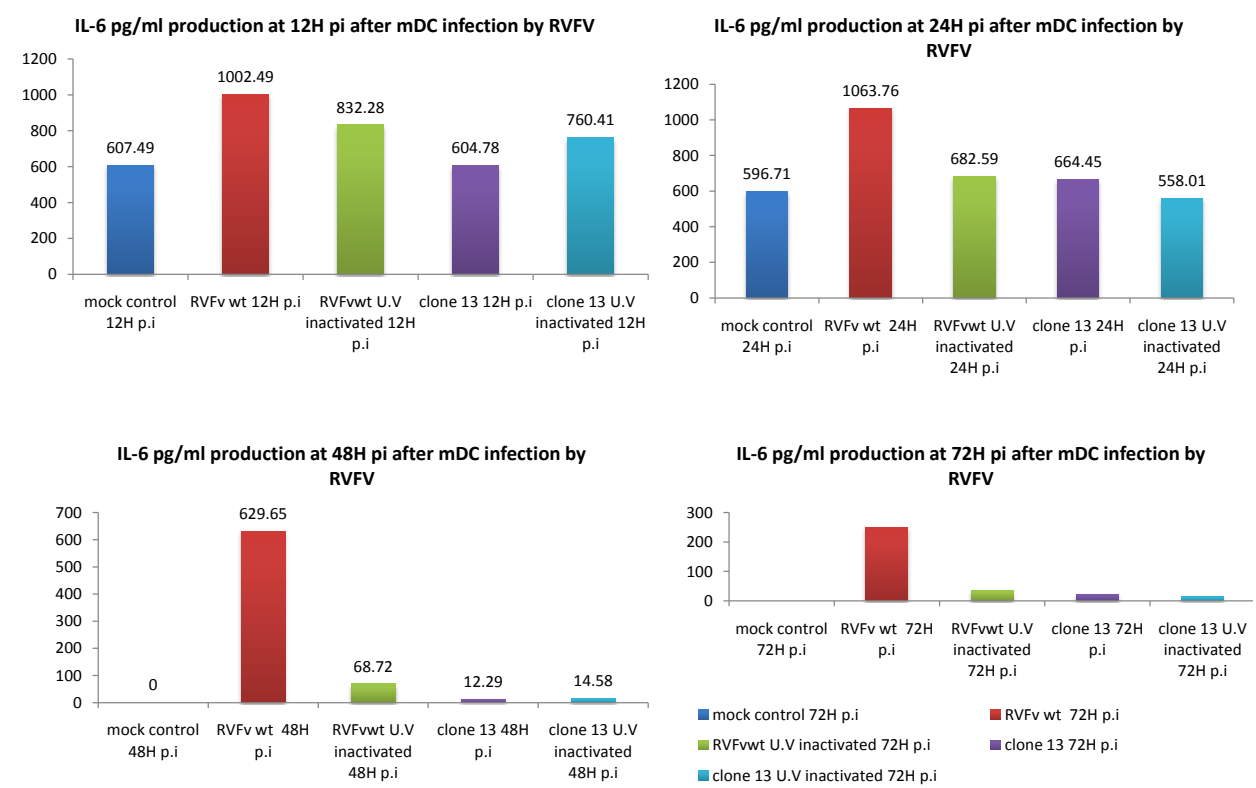

Figure 14. IL-6 production of human mDCs after infection with RVFV. 

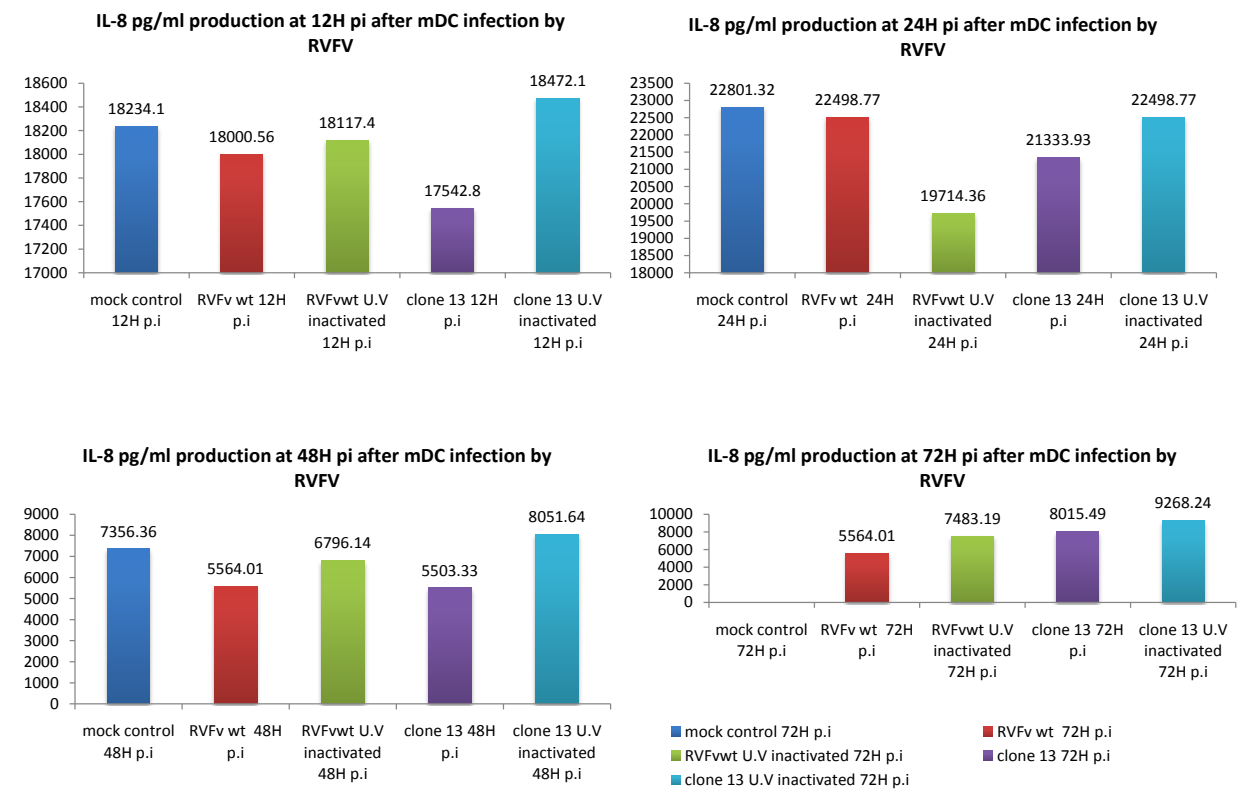

Figure 15. IL-8 production of human mDCs after infection with RVFV.

\subsubsection{Proinflammatory cytokine production in pDCs infected by RVFV}

Because pDCs produce large amounts of cytokines, particularly type I interferons, they regulate inflammation and link innate with adaptive immunity. A very important aspect of pDC mediated regulation of adaptive immunity is the ability, through the production of both type I interferon and IL-6, to induce human B cells to differentiate into plasma cells and produce immunoglobulin [108]. Figure 16 and Figure 31 to Figure 35 in the appendix show the results of the analysis of the expression of proinflammatory cytokines (IL-1 $\beta$, IL-6, IL-8, IL-12p70, TNF $\alpha$, and IL-10) in pDCs infected with wtRFV ZH548 (red bar), with UV inactivated wtRVFV ZH548 (green bar), RVFV clone 13 (purple bar), UV inactivated RVFV clone 13 (light blue bar), and mock control (dark blue bar) at 12h, 24h, 48h, and $72 \mathrm{~h}$ (clockwise). 

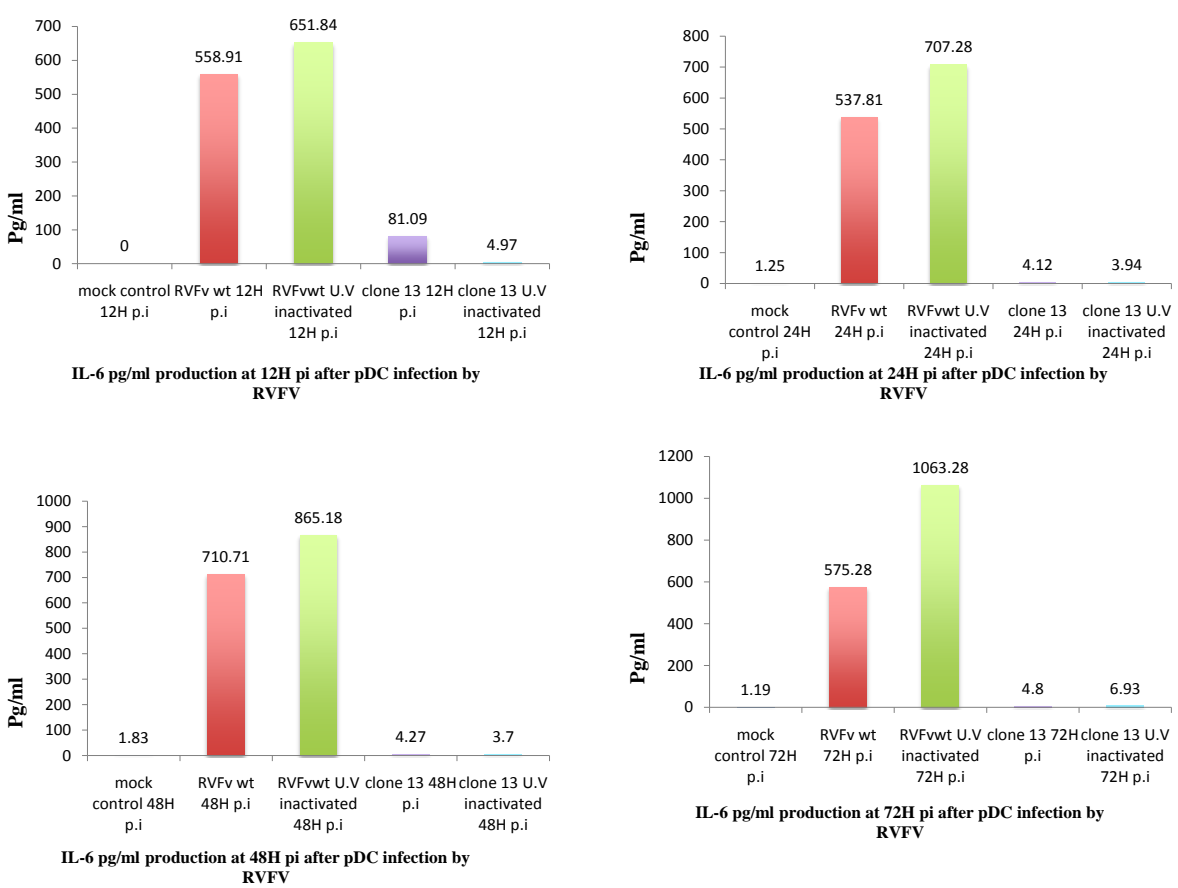

Figure 16. IL-6 production of human pDCs after infection with RVFV at $12 \mathrm{~h}, 24 \mathrm{~h}, 48 \mathrm{~h}$, and $72 \mathrm{~h}$ (clockwise).

The results for IL-1 $\beta$ (Figure 31) show the same level of production in all cases. Therefore the infection of pDCs with RVFV did not have an effect on IL-1 $\beta$ induction.

IL-6 production of pDCs (Figure 16) incubated with replication incompetent UV inactivated wtRVFV ZH548 was very pronounced with more than $1000 \mathrm{pg} / \mathrm{ml}$ produced at a very late time point ( $72 \mathrm{~h}$ post infection), and less than $600 \mathrm{pg} / \mathrm{ml}$ when $\mathrm{pDCs}$ were infected by replication competent wtRVFV ZH548. There was no IL-6 production in pDCs infected with mock control or replication competent RVFV clone 13 and replication incompetent UV inactivated RVFv clone 13.

IL-8 production showed no difference in pDCs (Figure 32) infected with the replication competent wtRVFV ZH548, RVFV clone 13, or when incubated with the correspondant UV inactivated replication incompetent supernatants and the mock control (less than $2,5 \mathrm{pg} / \mathrm{ml})$.

IL-10 was not produced in pDCs (Figure 33) infected with RVFV during late time point 48 and $72 \mathrm{~h}$ post infection, and produced at less than $2 \mathrm{pg}$ at 12 and $24 \mathrm{~h}$ post infection. 
For TNFa (Figure 34) and IL-12p70 (Figure 35) the results show no difference in production in all cases of infection. However IL-12p70 (Figure 35) was always secreted and transiently upregulated $12 \mathrm{~h}$ after infection with replication competent RVFV clone 13.

Altogether the results show only a sustained increase in IL-6 production in RVFV infected pDCs as well as mDCs. This is in line with observations that phlebovirus infection in mice using Punta Toro virus (PTV) as model virus leads to strong IL-6 induction [109].

\subsection{Effect of IL-6 on RVFV replication}

In chapter 3.2 it was demonstrated that DCs infected by wtRVFV ZH548 don't produce IFN- $\beta$. In chapter 3.6 the test of permissiveness showed that RVFV doesn't replicate in pDCs. In chapter 3.10 results indicated that pDCs infected with wtRVFVZH548, produce high amounts of IL-6. To test if IL-6 is the pro-inflammatory cytokine, which may block the replication of RVFV in pDCs, a TCID50 was designed using Vero E6 cells as indicator cells because RVFV replicates efficiently in these cells.

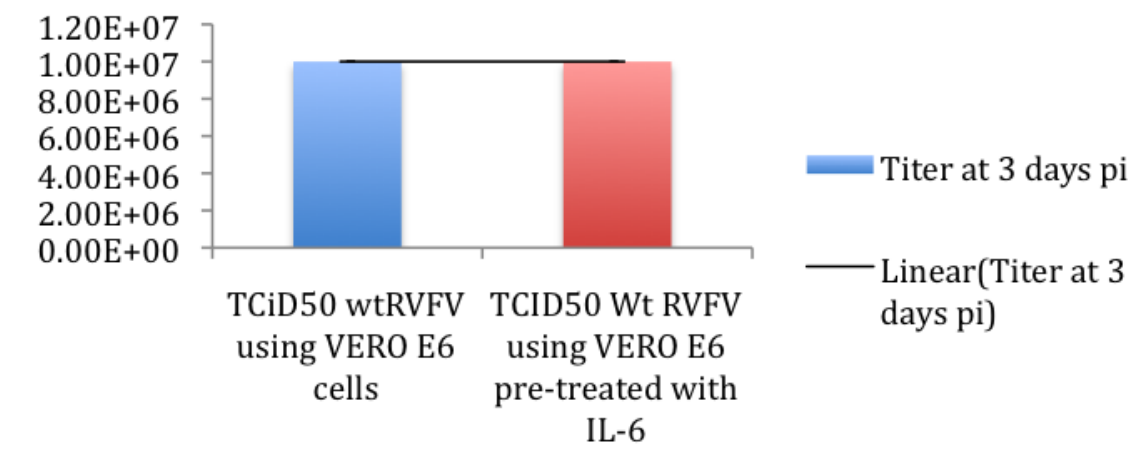

Figure 17. TCID50 of wtRVFVZH548 in Vero E6 and Vero E6 pre-treated with IL-6. Vero E6 infected with wtRVF ZH548 (blue bar), Vero E6 pretreated with IL-6 before infecting with wtRVF ZH548 (red bar).

Our results do not show a difference between RVFV replication measured by TCID50 using Vero E6 cells or Vero E6 pre-treated with IL-6 (Figure 17). IL-6 doesn't seem to be responsible for an inhibition of the wtRVFV ZH 548 replication in pDCs. 


\subsection{Interferon- $\alpha$ production of DCs infected with RVFV}

During viral infection, some of the most prominent cytokines produced are the type I interferons [103]. Nothing was known before about type I IFN production in DCs after infection with RVFV. It was already known that wtRVFV-ZH548 interfere with IFN- $\beta$ induction and clone 13 is known to induce productively IFN- $\beta$ in epithelial cells and fibroblasts. To check for IFN- $\alpha$ production of DCs during RVFV infection supernatants from pDCs and mDCs incubated with wtRVFV ZH548, UV inactivated wtRVFV ZH548, RVFV clone 13, UV inactivated RVFV clone 13 , and a mock control collected at $12 \mathrm{~h}$, 24h, 48h, and 72h p.i were tested using the Human IFN- $\alpha$ Elisa kit (Table 6) to quantify IFN- $\alpha$ in each of these samples.

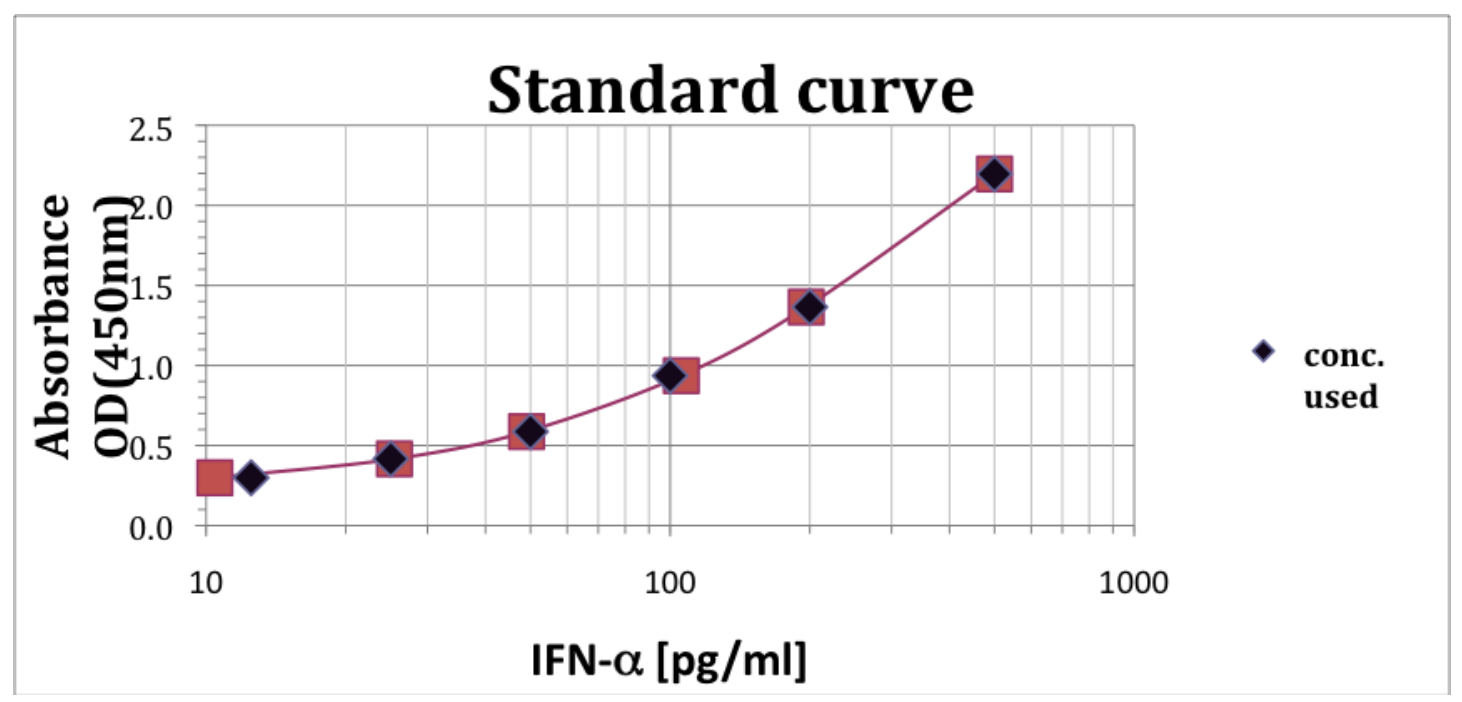

Figure 18. Representation of the high sensitivity standard curve shows that the test was well fitted. There is no variation from this typical curve provided. Concentration used (black dots), Concentration calculated (orange rectangles). The back fit concentration extrapolated from the standard curve seems to be very accurate for determination of the sample titer and the performance of the kit. 


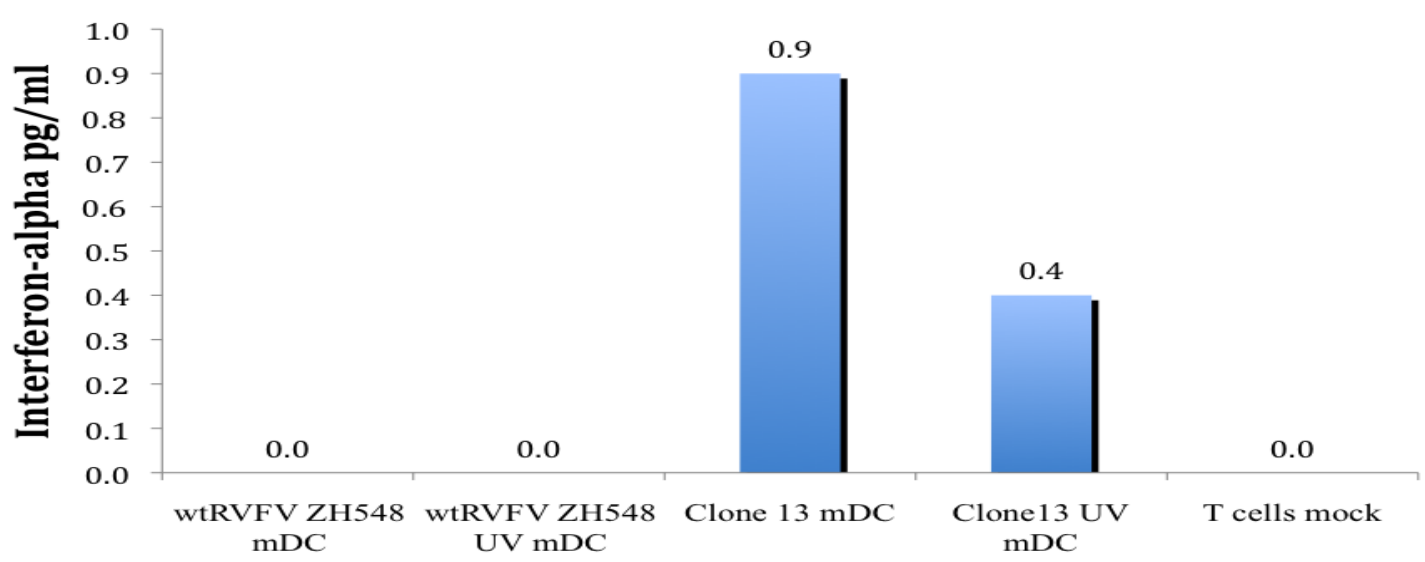

Figure 19. IFN- $\alpha$ secretion in supernatant harvested at $72 \mathrm{~h}$ p.i from mDCs infected with the replication competent wtRVF ZH548, naturally avirulent strain clone 13, UV inactivated wtRVF ZH548, and supernatant of non infected Vero E6 cell as control.

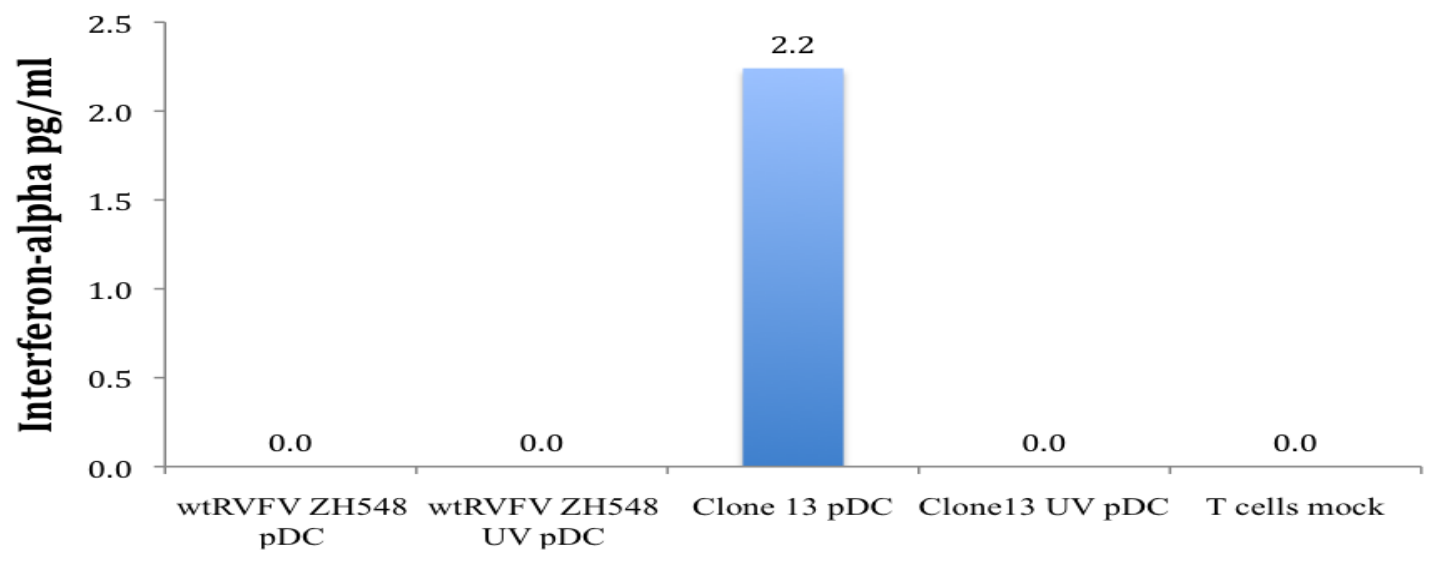

Figure 20. IFN- $\alpha$ secretion in supernatant harvested at $72 \mathrm{~h}$ p.i from pDCs infected with the replication competent wtRVF ZH548, naturally avirulent strain clone 13, UV inactivated wtRVF ZH548, and supernatant of non infected Vero E6 cell as control.

Until $72 \mathrm{~h}$ post infection a nearly complete absence of IFN- $\alpha$ production was observed (Figure 19). The highest amount measured was 0.9 and $0,4 \mathrm{pg} / \mathrm{ml}$ in $\mathrm{mDC}$ and 2,2 pg/ml in pDC after infection with RVFV clone 13. These amounts were below the lowest concentration of the standard curve $(12,5 \mathrm{pg} / \mathrm{ml})$ of the highly sensitive assay as shown by standards (Figure 18).

The results demonstrate that human $\mathrm{pDC}$ as well $\mathrm{mDC}$ were unable to induce IFN- $\alpha$ production when infected either with replication competent wtRVF ZH548 or clone13 and even when incubated with the corresponding non replication competent UV inactivated strains. 


\subsection{Comparison of IL-6 and IFN- $\alpha$ production of pDCs infected with RVFV} and TBEV

To exclude the possibility that the DCs infected with RVFV were functionally impaired due to unrecognized problems during isolation from buffy coat, infection with Tick-borne encephalitis virus (TBEV) was included as a positive control for DC function. TBEV induces an activation of DCs as well as IL- 6 and IFN- $\alpha$ production in pDCs and mDCs.

Supernatant of pDC cultures and mDC cultures incubated with replication competent and UV inactivated replication incompetent RVFV ZH548, RVFV clone 13, TBEV and a mock control, were harvested at different time points (12h, 24h, 48h, 72h p.i). Cytokines were tested as described in chapter 2.2.7.

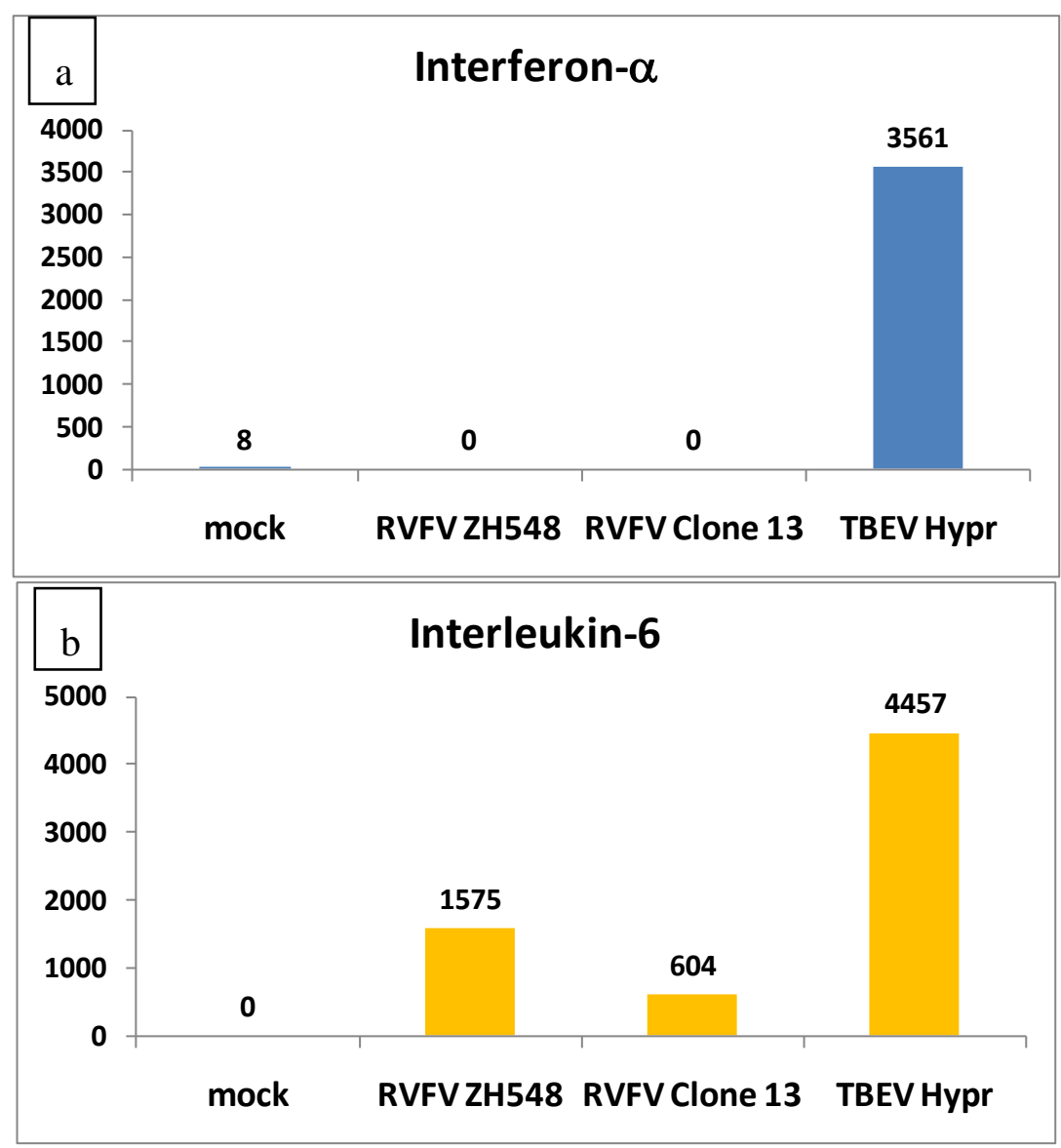

Figure 21. Comparison of the induction of cytokines in pDCs by RVFV and TBEV. High IL-6 production (a) was induced by replication competent RVFV (1574pg/ml) and TBEV (4457pg/ml) in pDCs but only TBEV shows IFN- $\alpha$ production (b) $(201 \mathrm{pg} / \mathrm{ml})$. 


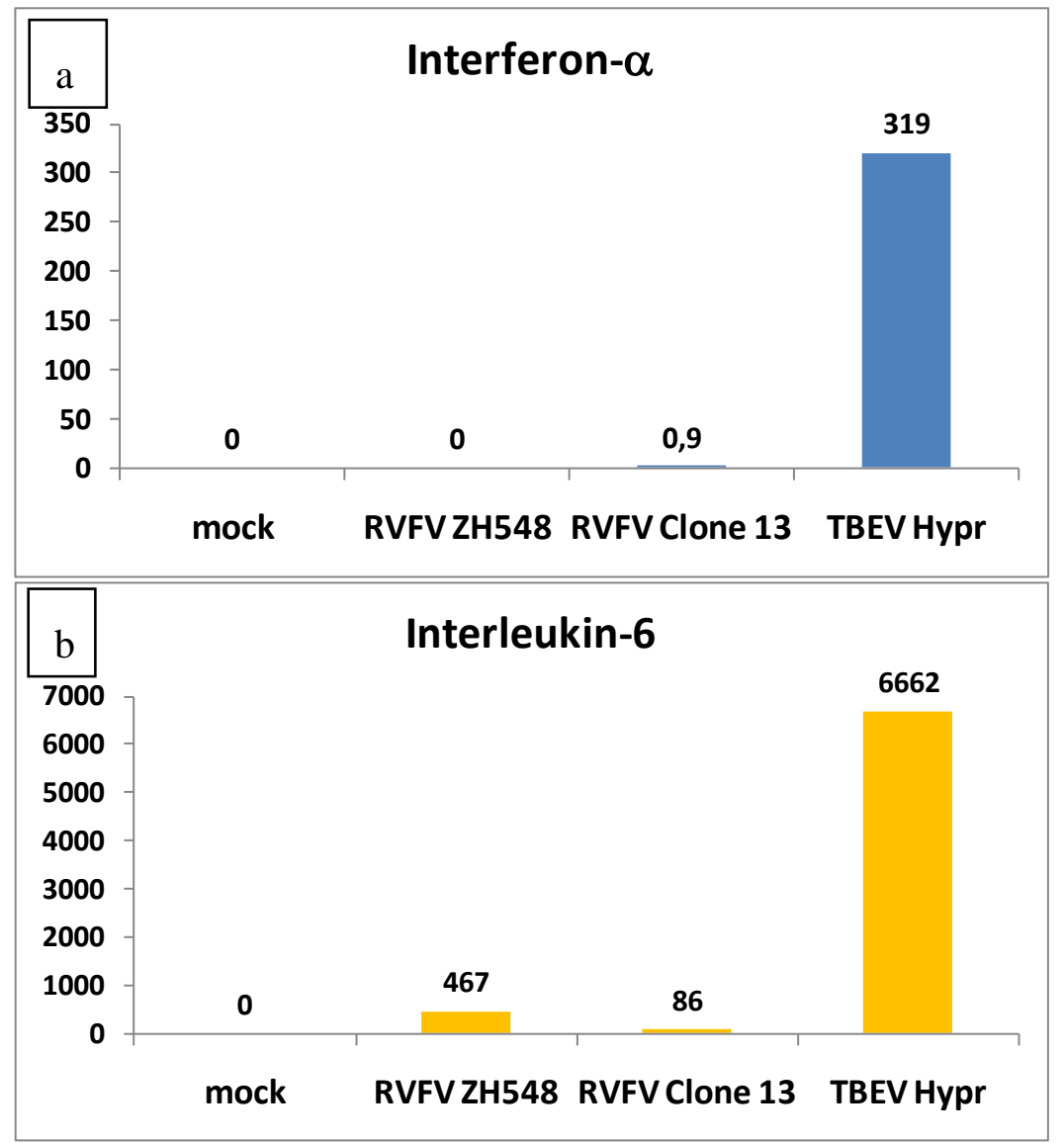

Figure 22. Induction of cytokines in mDCs by RVFV and TBEV. High IL-6 (a) production was induced by replication competent wtRVFV ZH548 in mDCs and weak IFN- $\alpha$ production (b) (less than 1pg/ml) with the replication competent clone $13(0,9 \mathrm{pg} / \mathrm{ml})$.

The results clearly show that both mDCs and pDCs responded to TBEV infection with strong IL-6 and IFN- $\alpha$ production indicating proper DC function. Therefore, the absence of IFN- $\alpha$ production as well as the isolated induction of IL-6 is characteristic for RVFV infections of DCs and not the consequence of a DC malfunction (arising from unrecognized problems during isolation).

\subsection{Expression and regulation of surface marker molecules in mDCs infected by RVFV}

Expression and regulation of DC surface molecule markers have been shown to have an effect on the mobilization of the adaptive immune response during viral infection. In order to investigate the effect of RVFV on surface marker induction in DCs. Surface molecules markers, CD80, CD86, CD40, CD83, CD58, CD54, CD95, MHCI, and MHCII 
were evaluated by FACS analysis using the Flo JO software. Fluorescent antibodies against the various surface markers were used (Table 7).

The markers CD1c and CD11c used as mDC positive control makers were all expressed, while CD14 used as a negative control marker showed downreguation following infection with wtRVF ZH548 or faint expression.

Figure 36 to Figure 44 in the appendix shows the regulation of surface markers after an infection of mDCs by RVFV. In all figures results are indicated as follows: infection with wtRVFV (light blue), UV inactivated wtRVFV (purple), RVFV clone 13 (dark blue), UV inactivated RVFV clone 13 (red), and mock control (orange) at 12h, 24h, 48h, and $72 \mathrm{~h}$ (clockwise).

At $12 \mathrm{~h}$ following incubation of mDCs with RVFV (Table 14), compared to the mock control, an upregulation of the maturation marker CD83 and the antigen presenting molecule MHCII was observed, whereas the apoptosis marker CD95, the co stimulator marker CD86 and the MHCI were downregulated by the replication competent wtRVFV ZH548. At that time, following incubation with the replication competent clone 13, a downregulation of CD86 and CD95 was observed. Whereas the co-stimulatory marker CD40 was downregulated following incubation with wtRVFV ZH548, clone 13, the UV inactivated wtRVF ZH548 and the UV inactivated clone 13. The other measured markers such as the adhesion markers CD54, CD58 showed an expression while the costimulatory marker CD80 was dowregulated. 
Table 14. Activation of surface molecule expression in mDCs $12 \mathrm{~h}$ post infection. Legend: -, no expression; + , expression; $\uparrow$ upregulated compared to mock infection; $\boldsymbol{\downarrow}$ downregulated compared to mock infection. The original FACS diagrams can be found in the appendix (Figure 36 to Figure 44).

\begin{tabular}{|c|c|c|c|c|c|}
\hline Marker & not infected & wtRVFV ZH548 & $\begin{array}{c}\text { RVFV ZH548 } \\
\text { UV-inactivated }\end{array}$ & RVFV clone 13 & $\begin{array}{l}\text { RVFV clone } 13 \\
\text { UV-inactivated }\end{array}$ \\
\hline CD1c & + & $\uparrow$ & + & + & + \\
\hline CD14 & + & $\downarrow$ & + & + & + \\
\hline CD11c & + & $\uparrow$ & + & + & + \\
\hline CD80 & - & - & - & - & - \\
\hline CD86 & + & $\downarrow$ & $\downarrow$ & $\downarrow$ & + \\
\hline CD40 & + & $\downarrow$ & $\downarrow$ & $\downarrow$ & $\downarrow$ \\
\hline CD83 & + & $\uparrow$ & + & + & + \\
\hline CD58 & + & + & + & + & + \\
\hline CD54 & + & + & + & + & + \\
\hline CD95 & + & $\downarrow$ & $\downarrow$ & $\downarrow$ & $\downarrow$ \\
\hline MHC-I & + & $\downarrow$ & + & + & + \\
\hline MHC-II & + & $\uparrow$ & + & + & + \\
\hline
\end{tabular}

At $24 \mathrm{~h}$ following incubation of mDCs with RVFV (Table 15) compared to the mock control a down-regulation of CD86 and CD95 using wtRVFV ZH548, clone 13, and their corresponding UV inactivated strains was observed. However CD86 shows an expression following UV inactivated clone 13 incubation. All the remaining measured markers CD54, CD58, CD83, MHCI, MHCII, CD1c, CD11c were expressed except the CD14, which did not show expression. 
Table 15. Activation of surface molecule expression in mDCs $24 \mathrm{~h}$ post infection. Legend: -, no expression; + , expression; $\uparrow$ upregulated compared to mock infection; $\downarrow$ downregulated compared to mock infection. The original FACS diagrams can be found in the appendix (Figure 36 to Figure 44).

\begin{tabular}{|c|c|c|c|c|c|}
\hline Marker & not infected & wtRVFV ZH548 & $\begin{array}{c}\text { RVFV ZH548 } \\
\text { UV-inactivated }\end{array}$ & RVFV clone 13 & $\begin{array}{l}\text { RVFV clone } 13 \\
\text { UV-inactivated }\end{array}$ \\
\hline CD1c & + & + & + & + & + \\
\hline CD14 & - & - & - & - & - \\
\hline CD11c & + & + & + & + & + \\
\hline CD80 & - & - & - & - & - \\
\hline CD86 & + & $\downarrow$ & $\downarrow$ & $\downarrow$ & + \\
\hline CD40 & - & + & + & + & + \\
\hline CD83 & + & + & + & + & + \\
\hline CD58 & + & + & $\downarrow$ & + & + \\
\hline CD54 & + & + & $\downarrow$ & + & + \\
\hline CD95 & + & $\downarrow$ & $\downarrow$ & $\downarrow$ & $\downarrow$ \\
\hline MHC-I & + & + & + & + & + \\
\hline MHC-II & + & + & + & + & + \\
\hline
\end{tabular}

At $48 \mathrm{~h}$ following incubation of $\mathrm{mDC}$ with RVFV (

Table 16), a downregulation of CD58 and CD54 using wtRVFV ZH548, wtRVF ZH548

UV inactivated was observed, whereas no expression observed with CD80, CD83, CD95.

However MHCI, MHCII, and CD40 were still expressed. 
Table 16. Activation of surface molecule expression in mDCs $48 \mathrm{~h}$ post infection. Legend: -, no expression; + , expression; $\uparrow$ upregulated compared to mock infection; $\downarrow$ downregulated compared to mock infection. The original FACS diagrams can be found in the appendix (Figure 36 to Figure 44).

\begin{tabular}{|c|c|c|c|c|c|}
\hline Marker & not infected & wtRVFV ZH548 & $\begin{array}{c}\text { RVFV ZH548 } \\
\text { UV-inactivated }\end{array}$ & RVFV clone 13 & $\begin{array}{l}\text { RVFV clone } 13 \\
\text { UV-inactivated }\end{array}$ \\
\hline CD1c & - & - & - & - & - \\
\hline CD11c & + & $\downarrow$ & + & + & + \\
\hline CD14 & + & - & $v$ & $\downarrow$ & + \\
\hline CD80 & - & - & - & - & - \\
\hline CD86 & + & + & + & + & + \\
\hline CD40 & + & + & + & + & + \\
\hline CD83 & - & - & - & - & - \\
\hline CD58 & + & $\downarrow$ & + & + & + \\
\hline CD54 & + & $\downarrow$ & $\downarrow$ & + & \\
\hline CD95 & - & - & - & - & - \\
\hline MHC-I & + & + & + & + & + \\
\hline MHC-II & + & + & + & + & + \\
\hline
\end{tabular}

At $72 \mathrm{~h}$ post infection there is no regulation observed. Only the positive control marker was downregulated following incubation of mDCs with wtRVFV ZH548. However CD58, CD86, CD95, MHCO, MHCII, CD40 were still expressed. 
Table 17. Activation of surface molecule expression in mDCs $72 \mathrm{~h}$ post infection. Legend: -, no expression; + , expression; $\uparrow$ upregulated compared to mock infection; $\downarrow$ downregulated compared to mock infection. The original FACS diagrams can be found in the appendix (Figure 36 to Figure 44).

$\begin{array}{lccccc}\text { Marker } & \text { not infected } & \text { wtRVFV ZH548 } & \text { RVFV ZH548 } & \text { RVFV clone 13 } & \text { RVFV clone 13 } \\ \text { CD1c } & - & - & \text { UV-inactivated } & & \text { UV-inactivated } \\ \text { CD11c } & + & \downarrow & - & + & + \\ \text { CD14 } & + & - & + & + & + \\ \text { CD80 } & - & + & + & + & + \\ \text { CD86 } & + & + & + & + & + \\ \text { CD40 } & + & - & + & + & + \\ \text { CD83 } & - & + & - & + & + \\ \text { CD58 } & + & - & + & + & + \\ \text { CD54 } & - & + & + & + & + \\ \text { CD95 } & + & + & + & + & + \\ \text { MHC-I } & + & + & + & + & + \\ \text { MHC-II } & + & + & + & +\end{array}$

\subsection{Expression and regulation of surface marker molecules in pDCs infected by RVFV}

In order to assay the ability of RVFV to induce activation and maturation of pDCs, pDCs were infected with wtRVFV ZH548 and the in vivo attenuated mutant RVFV clone 13. Surface molecules markers, CD83, CD80, CD86, CD54, CD58, CD95, MHCI, and MHCII were evaluated by FACS analysis using the Flo JO sofware. Fluorescent antibodies against the various surface markers were used (Table 7).

At $12 \mathrm{~h}$ following pDC infection using clone 13, an upregulation of CD83 and MHCI was observed. At that time point CD58, CD54, CD95 and CD40 were not expressed whereas CD80, CD86 and MHCII was observed but remained unchanged compared to mockinfected cells. 
Table 18. Activation of surface molecule expression in pDCs $12 \mathrm{~h}$ post infection. Legend: -, no expression; + , expression; $\uparrow$ upregulated compared to mock infection; $\downarrow$ downregulated compared to mock infection. The original FACS diagrams can be found in the appendix (Figure 45 to Figure 53).

\begin{tabular}{|c|c|c|c|c|c|}
\hline Marker & not infected & wtRVFV ZH548 & $\begin{array}{c}\text { RVFV ZH548 } \\
\text { UV-inactivated }\end{array}$ & RVFV clone 13 & $\begin{array}{l}\text { RVFV clone } 13 \\
\text { UV-inactivated }\end{array}$ \\
\hline CD123 & + & + & + & + & + \\
\hline CD80 & + & + & + & + & + \\
\hline CD86 & + & + & + & + & + \\
\hline CD40 & - & - & - & - & - \\
\hline CD83 & + & + & + & $\uparrow$ & + \\
\hline CD58 & - & - & - & - & - \\
\hline CD54 & - & - & - & - & - \\
\hline CD95 & - & - & - & - & - \\
\hline MHC-I & - & - & - & $\uparrow$ & - \\
\hline MHC-II & + & + & + & + & + \\
\hline
\end{tabular}

The original FACS diagrams can be found in the appendix (Figure 45 to Figure 53).

At 24h following incubation of pDCs with RVFV, a downregulation of CD80 was observed except for UV inactivated clone 13, whereas CD86 was downregulated in all cases. CD58 was only downregulated when using the replication incompetent UV inactivated wtRVFV ZH548 and UV inactivated clone 13. However CD54, CD83, CD95, MHCII were well expressed and MHCI was upregulated following incubation using clone 13. 
Table 19. Activation of surface molecule expression in pDCs 24 h post infection. Legend: -, no expression; + , expression; $\uparrow$ upregulated compared to mock infection; $\boldsymbol{\downarrow}$ downregulated compared to mock infection. Markers highlighted in the following groups: pDC markers, co-stimulatory markers, maturation marker, adhesion markers, apoptosis marker and antigen presenting molecules.

\begin{tabular}{|c|c|c|c|c|c|}
\hline Marker & not infected & wtRVFV ZH548 & $\begin{array}{c}\text { RVFV ZH548 } \\
\text { UV-inactivated }\end{array}$ & RVFV clone 13 & $\begin{array}{l}\text { RVFV clone } 13 \\
\text { UV-inactivated }\end{array}$ \\
\hline CD123 & + & + & + & + & + \\
\hline CD80 & + & $\downarrow$ & $\downarrow$ & $\downarrow$ & + \\
\hline CD86 & $\downarrow$ & $\downarrow$ & $\downarrow$ & $\downarrow$ & $\downarrow$ \\
\hline CD40 & - & - & - & - & - \\
\hline CD83 & + & + & + & + & + \\
\hline CD58 & + & + & $\downarrow$ & + & $\downarrow$ \\
\hline CD54 & + & + & + & + & + \\
\hline CD95 & + & + & + & + & + \\
\hline MHC-I & + & + & + & $\uparrow$ & + \\
\hline MHC-II & + & + & + & + & + \\
\hline
\end{tabular}

The original FACS diagrams can be found in the appendix (Figure 45 to Figure 53).

At $48 \mathrm{~h}$ following pDC infection with RVFV, downregulation of CD80 and CD86 was observed. CD54 was upregulated following clone 13 infection and CD58 was upregulated after incubation with UV-inactivated clone 13. CD54, CD83, CD95, MHCII remained unchanged compared to mock-infected cells. 
Table 20. Activation of surface molecule expression in pDCs $48 \mathrm{~h}$ post infection. Legend: -, no expression; + , expression; $\uparrow$ upregulated compared to mock infection; $\boldsymbol{\downarrow}$ downregulated compared to mock infection. Markers highlighted in the following groups: pDC markers, co-stimulatory markers, maturation marker, adhesion markers, apoptosis marker and antigen presenting molecules.

$\begin{array}{lccccc}\text { Marker } & \text { not infected } & \text { wtRVFV ZH548 } & \begin{array}{c}\text { RVFV ZH548 } \\ \text { UV-inactivated }\end{array} & \text { RVFV clone } 13 & \text { RVFV clone } 13 \\ \text { CD123 } & + & + & + & + & \text { UV-inactivated } \\ \text { CD80 } & + & \downarrow & \downarrow & \downarrow & + \\ \text { CD86 } & \downarrow & \downarrow & - & + & \downarrow \\ \text { CD40 } & - & - & + & + & + \\ \text { CD83 } & + & + & + & + & + \\ \text { CD58 } & + & + & + & + & + \\ \text { CD54 } & + & + & + & + & + \\ \text { CD95 } & + & + & + & + & + \\ \text { MHC-I } & + & + & + & + & + \\ \text { MHC-II } & + & + & & + & +\end{array}$

The original FACS diagrams can be found in the appendix (Figure 45 to Figure 53). At $72 \mathrm{~h}$ following pDc infection, an upregulation of CD54 after incubation with wtRVFV ZH548, wtRVFV ZH548 UV-inactivated and clone 13 UV-inactivated was observed compared to mock-infected DCs whereas following infection with the replication competent clone 13 CD54 upregulation was not observed. MCHI was only upregulated after infection with replication-competent clone 13. CD80 was downregulated for all virus preparations used compared to mock-infected pDCs. 
Table 21. Activation of surface molecule expression in pDCs $72 \mathrm{~h}$ post infection. Legend: -, no expression; + , expression; $\uparrow$ upregulated compared to mock infection; $\downarrow$ downregulated compared to mock infection. Markers highlighted in the following groups: pDC markers, co-stimulatory markers, maturation marker, adhesion markers, apoptosis marker and antigen presenting molecules.

\begin{tabular}{|c|c|c|c|c|c|}
\hline Marker & not infected & wtRVFV ZH548 & $\begin{array}{c}\text { RVFV ZH548 } \\
\text { UV-inactivated }\end{array}$ & RVFV clone 13 & $\begin{array}{l}\text { RVFV clone } 13 \\
\text { UV-inactivated }\end{array}$ \\
\hline CD123 & + & + & + & $\downarrow$ & + \\
\hline CD80 & + & $\downarrow$ & $\downarrow$ & $\downarrow$ & $\downarrow$ \\
\hline CD86 & - & - & - & - & - \\
\hline CD40 & - & - & - & - & - \\
\hline CD83 & - & - & - & - & - \\
\hline CD58 & + & + & + & + & + \\
\hline CD54 & + & 个 & 个 & $\downarrow$ & $\uparrow$ \\
\hline CD95 & + & + & + & + & + \\
\hline MHC-I & + & + & + & $\uparrow$ & + \\
\hline MHC-II & + & + & + & + & + \\
\hline
\end{tabular}




\section{$4 \quad$ Discussion}

A wide range of clinical symptoms has been described for RVFV infections in man ranging from harmless fever to severe haemorrhagic fever and blindness. In animals RVFV quickly replicates to very high titers [111]. Viral factors and a high genetic variability may be involved in the pathogenesis and explain the wide range of clinical outcome in man and animals. The differences in virulence may be due to the immune compromising effect of wtRVFV strains through a) the suppression of the type I IFN induction and $b$ ) the impairment of DC function.

In this project wtRVFV strains were monitored for their immunomodulatory effects by analysing the natural variability of NSs activity on the human IFN- $\beta$ promoter and by assessing the permissiveness of human DCs for wtRVFV strains.

Innate immune mechanisms have the potential to shape and regulate the evolving adaptive immune response by promoting or inhibiting development of specific downstream effector mechanisms. Therefore the cytokine production and the type I interferon production of DCs exposed to RVFV was analysed.

The effect of wtRVFV strains on DCs was monitored by FACS-analysis of DC specific markers and activation markers such as costimulatory molecules, maturation marker, adhesion molecules, apoptosis marker and antigen-presenting molecules.

\subsection{Natural variability of NSs function}

The nonstructural NSs protein of RVFV is a major virulence factor subverting the innate immune defences of the host [3]. To assess the natural variability of the NSs gene the genes of 26 RVFV strains were sequenced and the expression suppressing activity of each RVFV NSs [3] was tested in a cotransfection assay. Filament formation in the nucleus due to NSs polymerisation was also investigated by immune fluorescence.

\subsubsection{Rift Valley Fever NSs variants suppressing IFN- $\beta$}

The NSs cDNAs of 26 RVFV strains were expressed in the absence of other viral components and its effect on IFN- $\beta$ promoter activity was analyzed. Significant differences of the IFN- $\beta$ promoter activation in the presence of NSs from the RVFV isolates R2, R4, R5, R6, R7, R18, R23, R26, R27, R29, R30 were observed in this study 
when compared with the wild type RVFV ZH548-NSs. However all of these NSs clones were efficient inhibitors of IFN- $\beta$ promoter activation.

These results demonstrate that the ability to suppress IFN production cosegregates with an intact NSs gene and confirmed previous in vivo data [112]. Furthermore, the NSs clones with inhibitory effect on IFN- $\beta$ promoter activation as well as RVFV ZH548-NSs were able to decrease the activity of the constitutively active SV40 promoter as indicated by low renilla luciferase activities when compared with RVFV-NSs clone 13 (data not shown). This is in line with previous observations that RVFV-NSs affects RNA polymerase II mediated transcription [7-113].

\subsubsection{Rift Valley Fever NSs variants not suppressing IFN- $\beta$}

R18-NSs and R7-NSs were unable to suppress IFN- $\beta$ promoter activation and weren't detectable in immunofluorescence (Figure 11 and Figure 12). Both RVFV-NSs R18 and RVFV-NSs R7 have no inhibitory effect at all which is very similar to the NSs from the avirulent RVFV strain clone 13 carrying a major deletion in the S-segment. A follow up of viral RNA samples revealed that strain R18 is indeed a RVFV clone 13 isolate. Consequently the results for R18-NSs simply confirm the loss of function of clone 13NSs [1].

The luciferase reporter assay results for R18 confirm previous findings that RVFV clone 13 (lacking a functional NSs), but not wild type virus induces IFN- $\beta$ gene expression [7]. Previous work has shown that recombinant NSs of wild-type strain ZH548 forms filamentous structures in the nuclei of transiently transfected cells; these structures were identical to those observed in ZH548-infected cells [7]. In contrast, the truncated NSs of RVFV clone 13 was almost not detectable and mainly found in the cytoplasm [7]. Except for RVFV clone 13 and MP12- the NSs proteins of all the RVFV strains analyzed so far form filamentous structures in the nuclei of infected cells [114].

The follow up has not yet revealed the identity of the strain from which R7-NSs was amplified. R7-NSs truly shows a significant inability to suppress IFN- $\beta$ promoter activation induced by RNA from VSV-infected cells (Figure 11).

R7-NSs was not detectable in immunofluorescence although it had an intact FLAG-tag and showed a point mutation at nt 344 causing an amino acid change from leucine to proline $(\mathrm{L} \rightarrow \mathrm{P})$. Although R7-NSs had an intact C-terminal domain (amino acids 249 to 
265 of the NSs protein) required for oligomerisation and filament formation [2], as well as an intact FLAG-tag it failed to inhibit IFN- $\beta$ promoter activation, and wasn't detectable in IFA tests. The observed absence of filament formation as well as the loss of IFN- $\beta$ inhibition function can therefore be attributed to the R7-NSs sequence point mutation, nt $344(\mathrm{~L} \rightarrow \mathrm{P})$. This mutation seems to have a strong effect on the NSs function since NSs R7 no longer inhibits activation of the IFN- $\beta$ promoter (Figure 10).

In contrast, R8-NSs, which is identical to R7-NSs except for the mutation at nt 344 $(\mathrm{L} \rightarrow \mathrm{P})$, is an efficient inhibitor of IFN- $\beta$ promoter activation (Figure 10) and forms nuclear filaments (Figure 12j). The leucin to proline mutation at nucleotide position 344 in R7 probably leads to an unstable conformation of the otherwise quite stable RVF-NSs and may result in proteasomal degradation as it is the case for RVFV clone 13 NSs [1]. Therefore, the unique point mutation leading a leucine to proline exchange at amino acid position 344 in R7-NSs is critical for NSs function and stability and leads to effects comparable to the large in-frame deletion of clone 13 NSs.

Table 22. Summary of characteristics of RVFV NSs variants suppressing and not suppressing IFN- $\beta$

\begin{tabular}{|c|c|c|c|c|c|c|}
\hline $\begin{array}{l}\text { RVFV } \\
\text { strain }\end{array}$ & Characteristics & $\begin{array}{l}\text { IFN- } \beta \\
\text { suppression }\end{array}$ & $\begin{array}{l}\text { Flag-TAG } \\
\text { detection }\end{array}$ & $\begin{array}{l}\text { point } \\
\text { mutations }\end{array}$ & $\begin{array}{l}\text { C-domain } \\
\text { aa } 249-265\end{array}$ & $\begin{array}{l}\text { filament } \\
\text { formation }\end{array}$ \\
\hline ZH548 & wild type & + & & & intact & + \\
\hline MP12 & $\begin{array}{l}\text { vaccine candidate } \\
\text { (point mutations) }\end{array}$ & + & & $\begin{array}{l}\text { nt } 513 \\
A \rightarrow V\end{array}$ & intact & $+/-$ \\
\hline $\begin{array}{l}\text { clone } \\
13\end{array}$ & $\begin{array}{l}\text { vaccine candidate } \\
\text { (deletion) }\end{array}$ & - & & & intact & - \\
\hline R18 & this study & - & - & & intact & - \\
\hline R7 & this study & - & - & $\begin{array}{l}\text { nt } 344 \\
L \rightarrow P\end{array}$ & intact & - \\
\hline R10 & this study & + & + & $\begin{array}{l}\text { nt } 320 \\
L \rightarrow P\end{array}$ & intact & - \\
\hline $\mathbf{R 8}$ & this study & + & + & & intact & + \\
\hline
\end{tabular}


NSs-R10 has a point mutation at nt $320(\mathrm{~L} \rightarrow \mathrm{P})$. Immunofluorescence analysis revealed that this point mutation is sufficient to destroy the ability of NSs to form filamentous structure in the nucleus of infected cell. In contrast to R7, R10 however suppresses IFN- $\beta$ promoter activation.

R10-NSs is very similar to the NSs of the chemically attenuated virus derived from the Egyptian wild-type isolate ZH548 which is RVFV MP12 [13]. In NSs-MP12 the change observed in the NSs coding region, of which two are silent and one, at position 513, modifies alanine into a valine is the only difference between the $\mathrm{S}$ segment of wtRVF ZH548 and MP12 [115].

Both NSs-R10 and NSs-MP12 are fully functional in terms of IFN- $\beta$ promoter inhibition but impaired in their ability to form nuclear filaments.

Therefore it can be concluded, that filament formation and IFN- $\beta$ promoter inhibition are separate functions. It was previously reported [2] that filament formation requires an intact C-terminal domain. The results clearly show that the $\mathrm{C}$-terminal domain alone is not sufficient, since a single amino acid exchange at position 115 (nt 344) abrogates filament formation.

Our results also indicate that an $\mathrm{N}$ terminal domain is critical for filament formation as seen with R10-NSs. Another very interesting aspect found during this study is that the mutation of NSs-R10 occurring at nucleotide position 320 leads (amino acid 107) to a loss of filament formation but not to a loss of function in the inhibition of the IFN- $\beta$ promoter activation. Whereas the mutation of NSs-R7 at nucleotide position 344 destroys filament formation and also the type I IFN induction function. Meaning that in other words we identified here the domain between the nucleotide positions 320 and 344, which is absolutely essential for NSs function as well as filament formation.

Further experiments should be done to investigate the critical position for NSs interaction with TFIIH subunits, especially p44. Strain R7 could also be a good vaccine candidate for trial immunization as has been done for MP12 and clone 13.

\subsection{Characterisation of the interaction of RVFV with antigen presenting cells}

Increasing knowledge about the immune response to RVFV is urgently needed to improve the development of effective vaccine development, treatment strategies and to gain knowledge about the mechanisms driving species-specific outcome of disease. 
Elucidating how RVFV affects dendritic cells (DCs) is of particular importance given the crucial role of the antigen-presenting cells in the initiation of immune responses. The cross-talk between RVFV and DCs of human peripheral blood are investigated to improve avenues for future research. Viruses can directly or indirectly modulate dendritic cells and thus interfere with immune responses. Conventional dendritic cells (cDCs) of which mDCs form a subset are mostly devoted to taking up antigen in their steady state and presenting it to $\mathrm{T}$ cells in their activated or mature state, whereas pDCs after uptake of antigen, detect nucleic acids of pathogens which activates massive IFN- $\alpha$ production. pDCs also process antigen into small peptides, expose them to the surface and present them to $\mathrm{T}$ cells although less efficient compared to cDCs. Interestingly, certain viruses are tropic for DCs. Human immunodeficiency virus (HIV), measles virus (MV) and herpesvirus which can survive and even replicate in DCs, inducing immunosuppression at the same time [116]. In patients infected with HSV, severity of disease was found to be inversely correlated to the number of circulating $\mathrm{pDCs}$ and to a blunted IFN- $\alpha$ release response during the early phases of infection [117]. However, there is no information on the susceptibility of DCs to RVFV infection. As yet nothing is known about the interaction of RVFV and DCs. We hypothesis that spreading of RVFV in infected organisms can be caused by replication of RVFV in migratory DCs. pDCs also mediate regulation of adaptive immunity through the production of both type I interferon and IL6 , to induce human B cells to differentiate into plasma cells and produce immunoglobulin $[108,118]$. At the outset the hypothesis was that the type of proinflammatory cytokine produced might determine the outcome of RVFV infection. For that reason, the cytokines involved in inflammation (IL-1 $\beta$, IL-6, IL-8, IL-12p70, TNF $\alpha$, and IL-10) were investigated in vitro using freshly isolated human $\mathrm{mDCs}$ and $\mathrm{pDCs}$ to better understand the pathogenicity of RVFV. As yet nothing is known about IFN- $\alpha$ production by RVFVinfected dendritic cells. For this reason we also investigated the IFN- $\alpha$ levels in vitro after infection of DCs with RVFV. 


\subsubsection{Replication of RVFV in dendritic cells and cytokine profile induced}

During this study DCs were exposed to RVFV infection. Human mDCs and pDCs utilized during our isolation experiments were collected from peripheral blood mononuclear cells (see chapters 2.2.4.1 and 2.2.4.2) and used for infection with RVFV. The results indicate for the first time that RVFV does replicate productively in $\mathrm{mDCs}$ but does not replicate in pDCs. The ability of RVFV to replicate in $\mathrm{mDCs}$ may constitute an advantage for the virus. After primary infection of peripheral cells uptake by and replication in migratory $\mathrm{mDCs}$ may facilitate the spread of the virus thus allowing systemic infection.

The study on proinflammatory cytokines showed only a production and an increase of IL6 in pDCs and mDCs exposed to RVFV. In contrast, neither inflammatory IL-8, TNF $\alpha$, IL-12p70 and IL-1 $\beta$ nor anti-inflammatory IL-10 were consistently detected.

Given the fact that $\mathrm{pDCs}$ fail to produce IFN- $\alpha$ in response to RVFV infection (see chapter 4.2.2), the absence of RVFV replication in pDCs is surprising. The most simple explanation for this phenomenon is the hypothesis that pDCs do not express the so far unidentified cellular receptor for RVFV and that pDCs have been reported to not endocytose antigens as efficiently as mDCs [76].

As a consequence RVFV could neither enter the cells nor could it induce IFN- $\alpha$ synthesis. RVFV however infects a very broad range of cell types of very different species (e.g. insects, ruminants and humans). Therefore it is highly likely that a generic component in the cellular membrane, which is absolutely essential for all cell types including pDCs, acts as a receptor for RVFV. Therefore, RVFV may be able to enter pDCs but replication is blocked by induction of an antiviral active cytokine other than IFN- $\alpha$.

From the cytokines investigated in this study, only IL-6 production was induced in DCs by RVFV. However IL-6 had no impact on RVFV replication when tested in VeroE6 cells (Figure 17) indicating that IL-6 might not responsible for the observed replication block in pDCs. Both mDCs and pDCs infected with wtRVFV ZH548 produced sustained levels of IL-6, whereas only small levels were produced following RVFV clone 13 infection.

Strong IL-6 production was observed after Punta Toro Virus (PTV) infection in mice (a small animal model for phlebovirus infection), where IL-6 overexpression was 
contributing to increased mortality [119]. Indeed the association of exaggerated IL-6 production and lethal septic shock has been established [120]. TLR 3 deficient mice did produce minimal levels of IL-6 after infection and survived longer than the wild type mice [119]. Nevertheless moderate IL-6 levels seem to have some protective effects in phlebovirus infections since IL-6 -/- mice are highly susceptible to PTV infection [119]. The exact role of IL-6 production after RVFV infection therefore remains to be determined.

Similar to RVFV replication in mDCs was also observed after infection with SARS-CoV [121], whereas replication of SARS-CoV in pDCs was blocked. However in contrast to RVFV, pDCs are activated without virus replication and they induce IFN- $\alpha$ during SARS-CoV infection, which cause severe illness. Thus, the interaction of DCs with viruses may lead to chronic illness or death when the natural functions of DCs are outmanoeuvred or exploited by viruses [122].

It is known from the literature that human pDCs are potent producers of cytokines and chemokines, which may potentiate adaptive responses by attracting activated CD4+ and CD8+ T cells during an immune response [123] [124, 125]. pDCs mediated regulation of adaptive immunity is an ability for human B cell to produce immunoglobulin [126] through the production of type I IFN and IL-6.

The single production of IL-6 in our study in RVFV infected DCs is surprising and might be attributed to interference of RVFV with DC functions. IL-6 is known to induce many changes in various cell types including endothelial cells, hepatocytes and Kupffer cells [98] and mediate fever in the acute phase. It is accepted that IL-6 acts as a potent mediator of inflammation although it possesses immunosuppressive activity under certain conditions [120, 127]. The isolated and sustained production of IL-6 and the absence of IFN- $\alpha$ production (chapter 3.12) may contribute to severe cases of RVFV infections in humans, which can lead to encephalitis, retinitis and fatal hepatitis with haemorrhages [30]. The inflammatory properties of IL-6 associated with its property to mediate fever early in infection could also be correlated with the outcome of RVFV disease in ruminants such as the regularly observed abortion and high mortality rate observed in young lambs [4]. 
The interaction of replication competent wtRVFVZH548 and RVFV clone 13 with DCs does not cause replication in pDCs. However wtRVFVZH548 and not RVFV clone 13 replicates in mDCs. Apparently pDCs are not suitable for RVFV replication. The possible explanation for this observation is that cDCs probably sense RVFV like fibroblasts and epithelial cells via RLRs.

The observed absence of replication in pDCs can be attributed either to a block of uptake or a block of replication. However based on the activation pattern observed in pDCs after RVFV infection a block of replication is probably more likely and bases on the following observations: (i) upregulation of MHCI at $12 \mathrm{~h}$ p.i. after infection with clone 13 as an indirect evidence for endogenous antigen production or cross-presentation after antigen uptake, (ii) different activation patterns (Figure 52) for replication competent and UVinactivated viruses. However the IL-6 production found nevertheless appears as the key determinant factor in DCs infected with RVFV. Despite the lack of apparent productive infection of RVFV in pDCs, we have observed an upregulation of MHCII (chapter 3.10) and significant production of IL-6. Also we noted a similar IL-6 production in DCs incubated with replication competent RVFV and DCs incubated with UV inactivated virus. These observations would mean that RVFV virus antigen is sufficient to induce IL6 and productive replication is not required.

\subsubsection{The interferon response of dendritic cells infected with RVFV}

Viruses have developed several kinds of immune evasion strategies to proliferate within host cells. Their main target is the type I IFN response. The ability of pDCs to secrete type I interferons depends on cellular sensors that promptly detect the presence of DNA and RNA viruses.

Treatment of DCs with replication-competent wtRVFV ZH545 virus, and UV-inactivated virus, did not induce an IFN- $\alpha$ signal. However a faint signal (less than $1 \mathrm{pg} / \mathrm{ml}$ ) was observed with replication competent RVFV clone 13 and inactivated RVFV clone 13, suggesting that RVFV was still able to induce a weak amount of IFN- $\alpha$ expression. Initial experiments using strain wtRVFV ZH548 and the NSs-deficient RVFV clone 13 for infection of human pDCs indicated that the viruses were taken up but did not replicate in these cells (Figure 13) in pDCs neither the wt virus nor the mutant RVFV clone 13 
induced IFN- $\alpha$ expression although the mutant is a very potent IFN-inducer in epithelial cells.

Virus-infected cells synthesize and secrete type I interferons which circulate in the body and induce expression of antiviral mechanisms limiting viral growth and spread [52]. The NSs protein antagonism to interferon was demonstrated [6]. So far it was found that pDCs secrete high levels of IFN- $\alpha$ in response to viral infections and constitute the natural interferon production of the body in human [128]. It is known that pDCs once triggered are mostly responsible for the release of type I IFN [129], but nothing is known about IFN- $\alpha$ induction in DCs infected by RVFV.

To summarize, a faint IFN- $\alpha$ production of less than $1 \mathrm{pg} / \mathrm{ml}$ in $\mathrm{mDCs}$ infected with RVFV clone 13 but not with wtRVFV ZH548 was observed. There was none in pDCs. wtRVFV replicated productively in $\mathrm{mDCs}$ but was unable to replicate in pDCs although no type I IFN was induced in both DC subsets. These observations are potentiated by previous work done in an animal model observing that avirulent strains were able to induce an initial peak of IFN at a very early stage of infection, whereas virulent strains did not do so but induce it shortly before death, when the animal had a high viremia [130].

All this argues for inhibition of type I IFN production in spite of replication in mDCs, as it was observed for epithelial cells in fibroblasts [131], indicating the possible use of $\mathrm{mDCs}$ as means for systemic dissemination of the virus in the infected host.

\subsubsection{Activation patterns of dendritic cells infected with RVFV}

Viruses have acquired many different mechanisms to escape the immune attack of the host [132-134]. The outcome of viral disease is thought to be partly linked to the numbers of and balance between pDCs and cDCs [122]. Clinical data show that patients with HSV-associated acute retinal necrosis have lower numbers of peripheral blood pDCs and exhibit impaired immune responses [117]. Similar findings were obtained in patients infected with Dengue virus (DENV) [135]. Here, we investigated whether RVFV infection develops immune-evasion mechanisms to modulate the innate and the specific immune responses.

DCs were characterised by DC specific marker detection (CD1c, CD11c for mDCs / CD123 for pDCs). To study the influence of RVFV infection on DC function, the 
expression of activation markers such as costimulatory molecules (CD80, CD86, CD40), maturation marker (CD83), adhesion molecules (CD54, CD58), apoptosis marker (CD95) and antigen-presenting molecules (MHCI, MHCII) of infected DCs was analysed by FACS analysis (see chapter 2.2.5).

As a whole, DCs appeared not activated or in a stage of abrogated activation after RVFV infection. RVFV-infected DCs clearly showed a downregulation of the costimulatory CD86 (in mDCs) or some expression but no upregulation of CD80 and CD86 (in pDCs). The maturation marker CD83 was transiently upregulated in mDCs infected with wtRVFV ZH548 and in pDCs infected with RVFV clone 13.

The adhesion molecules showed either expression and downregulation towards the last time point (CD54 and CD58 in mDCs) or expression and slight upregulation towards the last time point (CD54 in pDCs). The apoptosis marker CD95 was clearly downregulated in $\mathrm{mDCs}$ but not at all expressed in pDCs. Expression of antigen presenting MHCI was downregulated in mDCs and only upregulated in pDCs infected with RVFV clone 13. MHCII however was upregulated in mDCs after infection but only expressed at low level in pDCs.

\subsubsection{Activation of adhesion markers}

The adhesion molecule markers CD58 and CD54 are involved in cell-cell interaction. CD58 is also known as lymphocyte function-associated antigen 3 (LFA-3) [136] expressed on antigen presenting cells (APC), and binds to the CD2 (aka, LFA-2) receptor found [137] on T cells. It initiates encounters between T-cells and APC before T-cell activation when T-cells are roam the lymph nodes looking at antigen presenting MHC complexes on the surface of APCs [137]. CD54, is an intercellular adhesion molecule (ICAM) expressed in lymphocytes which has a ligand for an integrin receptor continuously present in low concentrations on the membranes of leukocytes [138].

During our study CD58 and CD54 were well expressed, however they were not upregulated compared to mock infected cells indicating impaired activation of DCs after RVFV infection. 


\subsubsection{Activation of maturation marker CD83}

For CD83 an upregulation was observed in mDCs infected with wtRVFV ZH548 indicating induction of maturation as CD83 is the most reliable maturation marker for dendritic cells (DCs) [139]. Interestingly wtRVFV ZH548 failed to induce CD83 in pDCs where weak transient upregulation only occurred after infection with RBFV clone 13. These results are similar to those seen in the case of infection of immature dendritic cells (iDCs) with severe acute respiratory syndrome (SARS) Coronavirus in two others studies $[140,141]$. Initiation of maturation was also reported in immature dendritic cells infected by measles virus revealed by rapid upregulation of CD83 [116].

The transient upregulation of CD83 therefore indicates impaired activation of DCs after infection with RVFV.

\subsubsection{Activation of costimulatory markers}

Comparing pDCs and $\mathrm{mDCs}$, our results do not show a clear difference in the expression or regulating of these activation markers. The costimulatory molecules such as CD80, CD86 in general show minimal expression in pDCs [76, 78, 142]. However in measles virus infected iDCs rapid maturation is induced as revealed by the upregulation of CD40, CD80, CD86 and also the maturation marker CD83 following infection [116].

This supports the view that there is impaired activation of DCs upon infection with RVFV.

\subsubsection{Activation of MHC markers}

While MHCII was clearly upregulated in mDCs and expressed in pDCs infected with wtRVFV ZH548, MHCI was downregulated in mDCs and no upregulation was observed in pDCs.

In general pDCs show low MHC class II expression compared with mDCs. In contrast to freshly isolated pDCs, activated pDCs augment cell surface expression of MHC class II and costimulatory molecules $[76,78,79]$. The up-regulation of MHCII increases their T cell stimulatory ability. Accordingly, activated pDCs induce expansion of antigen-specific memory CD8+ $\mathrm{T}$ cell populations and $\mathrm{TH} 1 \mathrm{CD} 4+\mathrm{T}$ cell populations specific for endogenous antigens [80] and influenza virus [143]. 
DCs infected with RVFV showed lack of MHCI upregulation. Missing MHCI upregulation was also observed, in the case of SARS-CoV although SARS-CoV-infected immature DCs were otherwise clearly activated [121].

The upregulation of MHCII and missing MHCI upregulation in RVFV infected mDCs therefore indicates initial stages of activation. On the other hand MHCI has the crucial function to display foreign peptides to cytotoxic T cells (CTLs), which will become activated and kill the cell. Many malignant cancer cells show down-regulation of MHC I, giving transformed cells the advantage of being able to avoid normal immune surveillance designed to destroy transformed cells [144].

Similarly downregulation of MHCI therefore could help to conceal RVFV infection of DCs and systemic dissemination of RVFV. The described MHC expression pattern demands to investigate whether DCs infected RVFV can activate natural killer cells (NK).

In summary RVFV infects and replicates efficiently in $\mathrm{mDCs}$, and the infected $\mathrm{mDCs}$ display transient CD83 upregulation. The upregulation of MHCII in RVFV infected DCs indicates an uptake of the virus which then can be transferred to adaptive immune cells allowing a perfect viral invasion. The overall results however suggest that RVFV induced incomplete maturation of mDCs. Even the apathogenic clone 13 does not fully activate DCs however in contrast to RVFV ZH548 at least in pDCs it induces MHCI upregulation. Further experiments have to show if activation of $\mathrm{T}$ cells by RVFV-infected DCs is impaired or not. 


\section{Summary}

The objective of this thesis was to analyse the interaction of Rift Valley Fever virus (RVFV) with the human innate immunity.

In recent work it was shown that the RVFV protein NSs, a non-structural protein, has an inhibitory effect on the interferon response of the innate immune system by blocking the interferon- $\beta$ (IFN- $\beta$ ) promoter [145]. To assess the natural variability of the NSs activity the immune modulatory effects of twenty-six wild type RVFV strains isolated throughout Africa were characterized using molecular biological tools. The diversity of these strains was also assessed by sequencing the NSs genes and by looking at their polymerisation activity in the nucleus of Vero E6 cells.

It was revealed that RVFV NSs-R7 contains an additional mutation at position 344 ( $\mathrm{T}$ to C), which leads to the replacement of leucine by proline. This mutation seems to have a strong effect on the NSs function since NSs-R7 no longer inhibits activation of the IFN- $\beta$ promoter and does not show filamentous structure formation in the nucleus of infected cells. The mutation of NSs-R10, which occurs at nucleotide position 320 , leads to a loss of filament formation but not to a loss of function in the inhibition of IFN- $\beta$. The critical region between nucleotides 320 and 344 should be investigated as putative interaction domain for NSs interaction with TFIIH subunits, especially $\mathrm{p} 44$. The results also merit to investigate the eligibility of strain R7 as a vaccine candidate.

Experiments with myeloid and plasmacytoid dendritic cells (mDCs / pDCs) revealed for the first time that the wtRVFV ZH548 only replicates in mDCs. Incomplete activation of both pDCs and mDCs after RVFV infection was observed. High amounts of the proinflammatory cytokine IL-6 combined with the complete lack of type I IFN responses in both $\mathrm{pDCs}$ and $\mathrm{mDCs}$ might be responsible for the severe outcome of RVFV wt infections. The combination of downregulation of MHCI and IFN- $\alpha$ indicate that RVFV may use dendritic cells for systemic dissemination.

Further investigations will need to focus on the interaction of dendritic cells with T-helper cells and cytotoxic T cells and their role in the putative dissemination of RVFV. 


\section{Bibliography}

1. Vialat, P., et al., The S segment of rift valley fever phlebovirus (Bunyaviridae) carries determinants for attenuation and virulence in mice. J Virol, 2000. 74(3): p. 1538-43.

2. Yadani, F.Z., et al., The carboxy-terminal acidic domain of Rift Valley Fever virus NSs protein is essential for the formation of filamentous structures but not for the nuclear localization of the protein. J Virol, 1999. 73(6): p. 5018-25.

3. Billecocq, A., et al., NSs protein of Rift Valley fever virus blocks interferon production by inhibiting host gene transcription. J Virol, 2004. 78(18): p. 9798806.

4. Daubney, R., J.R. Hudson, and P.C. Graham, Enzootic hepatitis of Rift Valley fever, an undescribed virus disease of sheep, cattle and man from East Africa. Journal of Pathology and Bacteriology, 1931. 34: p. 545-79.

5. Swanepoel, R. and N.K. Blackburn, Demonstration of nuclear immunofluorescence in Rift Valley fever infected cells. J Gen Virol, 1977. 34(3): p. 557-61.

6. Billecocq, A., et al., NSs protein of rift valley fever virus blocks interferon production by inhibiting host gene transcription. Journal of Virology, 2004. 78(18): p. 9798-9806.

7. Le May, N., et al., TFIIH transcription factor, a target for the Rift Valley hemorrhagic fever virus. Cell, 2004. 116(4): p. 541-550.

8. Swiss-Institute-of-Bioinformatics. ViralZone: Phlebovirus. 2010; Available from: http://www.expasy.org/viralzone/all_by_species/252.html.

9. Muller, R., et al., Characterization of clone 13, a naturally attenuated avirulent isolate of Rift Valley fever virus, which is altered in the small segment. Am J Trop Med Hyg, 1995. 53(4): p. 405-11.

10. Ihara, T., H. Akashi, and D.H.L. Bishop, Novel Coding Strategy (Ambisense Genomic Rna) Revealed by Sequence Analyses of Punta Toro Phlebovirus-S Rna. Virology, 1984. 136(2): p. 293-306.

11. Giorgi, C., et al., Sequences and Coding Strategies of the S-Rnas of Toscana and Rift-Valley Fever Viruses Compared to Those of Punta-Toro, Sicilian Sandfly Fever, and Uukuniemi Viruses. Virology, 1991. 180(2): p. 738-753.

12. Muller, R., et al., Characterization of Clone-13, a Naturally Attenuated Avirulent Isolate of Rift-Valley Fever Virus, Which Is Altered in the Small Segment. American Journal of Tropical Medicine and Hygiene, 1995. 53(4): p. 405-411.

13. Caplen, H., C.J. Peters, and D.H.L. Bishop, Mutagen-Directed Attenuation of RiftValley Fever Virus as a Method for Vaccine Development. Journal of General Virology, 1985. 66(Oct): p. 2271-2277.

14. Smithburn, K.C., Rift Valley Fever - the Neurotropic Adaptation of the Virus and the Experimental Use of This Modified Virus as a Vaccine. British Journal of Experimental Pathology, 1949. 30(1): p. 1-16. 
15. Sall, A.A., et al., Variability of the NSS protein among Rift Valley fever virus isolates. Journal of General Virology, 1997. 78: p. 2853-2858.

16. Bird, B.H., et al., Complete genome analysis of 33 ecologically and biologically diverse Rift Valley fever virus strains reveals widespread virus movement and low genetic diversity due to recent common ancestry. J Virol, 2007. 81(6): p. 2805-16.

17. Imam, I.Z. and M.A. Darwish, A preliminary report on an epidemic of Rift Valley Fever (RVF) in Egypt. J Egypt Public Health Assoc, 1977. 52(6): p. 417-8.

18. Abd el-Rahim, I.H., U. Abd el-Hakim, and M. Hussein, An epizootic of Rift Valley fever in Egypt in 1997. Rev Sci Tech, 1999. 18(3): p. 741-8.

19. Jouan, A., et al., [Evaluation of indicators of health in the area of Trarza during the epidemic of Rift Valley fever in 1987]. Bull Soc Pathol Exot, 1990. 83(5): p. 621-7.

20. Madani, T.A., et al., Rift Valley fever epidemic in Saudi Arabia: epidemiological, clinical, and laboratory characteristics. Clin Infect Dis, 2003. 37(8): p. 1084-92.

21. Thiongane, Y., et al., [Recent data on Rift Valley Fever epidemiology in Senegal]. Dakar Med, 1996. Spec No: p. 1-6.

22. CDC, Rift valley fever distribution. 2007.

23. Logan, T.M., et al., Rift Valley fever antibody in human sera collected after an outbreak in domestic animals in Kenya. Trans R Soc Trop Med Hyg, 1992. 86(2): p. 202-3.

24. Hoch, A.L., T.P. Gargan, 2nd, and C.L. Bailey, Mechanical transmission of Rift Valley fever virus by hematophagous Diptera. Am J Trop Med Hyg, 1985. 34(1): p. 188-93.

25. Chambers, P.G. and R. Swanepoel, Rift valley fever in abattoir workers. Cent Afr J Med, 1980. 26(6): p. 122-6.

26. LaBeaud, A.D., et al., Spectrum of Rift Valley fever virus transmission in Kenya: insights from three distinct regions. Am J Trop Med Hyg, 2007. 76(5): p. 795800 .

27. LaBeaud, A.D., et al., Interepidemic Rift Valley fever virus seropositivity, northeastern Kenya. Emerg Infect Dis, 2008. 14(8): p. 1240-6.

28. Olaleye, O.D., et al., Rift Valley fever in Nigeria: infections in humans. Rev Sci Tech, 1996. 15(3): p. 923-35.

29. Hoogstraal, H., et al., The Rift Valley fever epizootic in Egypt 1977-78. 2. Ecological and entomological studies. Trans R Soc Trop Med Hyg, 1979. 73(6): p. 624-9.

30. Woods, C.W., et al., An outbreak of Rift Valley fever in Northeastern Kenya, 1997-98. Emerg Infect Dis, 2002. 8(2): p. 138-44.

31. Linthicum, K.J., et al., Climate and satellite indicators to forecast Rift Valley fever epidemics in Kenya. Science, 1999. 285(5426): p. 397-400.

32. Zeller, H.G., et al., Enzootic activity of Rift Valley fever virus in Senegal. American Journal of Tropical Medicine and Hygiene, 1997. 56(3): p. 265-272.

33. Meegan, J.M., et al., Monoclonal-Antibodies to Identify Zinga Virus as Rift-Valley Fever Virus. Lancet, 1983. 1(8325): p. 641-641. 
34. Saluzzo, J.F., et al., [Rift Valley fever in Western Africa]. Rev Elev Med Vet Pays Trop, 1987. 40(3): p. 215-23.

35. Saluzzo, J.F., et al., Focus of Rift-Valley Fever Virus Transmission in Southern Mauritania. Lancet, 1987. 1(8531): p. 504-504.

36. Guillaud, M., et al., [Prevalence of antibodies against Rift Valley fever virus in sheep and goats in Senegal]. Ann Inst Pasteur Virol, 1988. 139(4): p. 455-9.

37. Trouillet, J., et al., Phlebotomes (Diptera :Psychodidae) du Senegal. Peuplement du Ferlo. Isolement d'arbovirose. Parasite 1995. 2: p. 289-296.

38. Saluzzo, J.F., et al., Focus of Rift Valley fever virus transmission in southern Mauritania. Lancet, 1987. 1(8531): p. 504.

39. FAO. Recognizing rift valley fever. Available from: http://www.fao.org/docrep/006/y4611e/y4611e04.htm.

40. Gargan, T.P., et al., Vector Potential of Selected North-American Mosquito Species for Rift-Valley Fever Virus. American Journal of Tropical Medicine and Hygiene, 1988. 38(2): p. 440-446.

41. Peters, C.J., Viral hemorrhagic fevers as bioterrorist threats, in Douglas and Bennett's Principles and Practice of Infectious Diseases, G.L. Mandell, R.G. Douglas, and J.E. Bennett, Editors. 2005, Elsevier/Charchill Livingstone: New York. p. 3626-3629.

42. Sall, A.A., et al., Origin of 1997-98 Rift Valley fever outbreak in East Africa. Lancet, 1998. 352(9140): p. 1596-7.

43. Sall, A.A., et al., Genetic reassortment of Rift Valley fever virus in nature. Journal of Virology, 1999. 73(10): p. 8196-8200.

44. Thiongane, Y., et al., Changes in Rift-Valley Fever Neutralizing Antibody Prevalence among Small Domestic Ruminants Following the 1987 Outbreak in the Senegal River Basin. Research in Virology, 1991. 142(1): p. 67-70.

45. Zeller, H.G., et al., Rift Valley fever antibody prevalence in domestic ungulates in Cameroon and several west African countries (1989-1992) following the 1987 Mauritanian outbreak. Res Virol, 1995. 146(1): p. 81-5.

46. Akira, S., S. Uematsu, and O. Takeuchi, Pathogen recognition and innate immunity. Cell, 2006. 124(4): p. 783-801.

47. Beutler, B.A., TLRs and innate immunity. Blood, 2009. 113(7): p. 1399-1407.

48. West, A.P., A.A. Koblansky, and S. Ghosh, Recognition and signaling by toll-like receptors. Annu Rev Cell Dev Biol, 2006. 22: p. 409-37.

49. van Vliet, S.J., et al., Innate signaling and regulation of dendritic cell immunity. Current Opinion in Immunology, 2007. 19(4): p. 435-440.

50. Malmgaard, L., Induction and regulation of IFNs during viral infections. Journal of Interferon and Cytokine Research, 2004. 24(8): p. 439-454.

51. Samuel, C.E., Antiviral actions of interferons. Clin Microbiol Rev, 2001. 14(4): p. 778-809, table of contents.

52. Haller, O., G. Kochs, and F. Weber, The interferon response circuit: Induction and suppression by pathogenic viruses. Virology, 2006. 344(1): p. 119-130. 
53. Garcia-Sastre, A., Inhibition of interferon-mediated antiviral responses by influenza A viruses and other negative-strand RNA viruses. Virology, 2001. 279(2): p. 375-84.

54. Chang, H.W., J.C. Watson, and B.L. Jacobs, The E3L gene of vaccinia virus encodes an inhibitor of the interferon-induced, double-stranded RNA-dependent protein kinase. Proc Natl Acad Sci U S A, 1992. 89(11): p. 4825-9.

55. Xiang, Y., et al., Blockade of interferon induction and action by the E3L doublestranded RNA binding proteins of vaccinia virus. J Virol, 2002. 76(10): p. 5251-9.

56. Charles, E.S., Innate Immunity Minireview Series: Making Biochemical Sense of Nucleic Acid Sensors That Trigger Antiviral Innate Immunity J. Biol. Chem, 2007. 282(21): p. 15313-15314.

57. Le May, N., et al., A SAP30 complex inhibits IFN-beta expression in Rift Valley fever virus infected cells. PLoS Pathog, 2008. 4(1): p. e13.

58. Agalioti, T., et al., Ordered recruitment of chromatin modifying and general transcription factors to the IFN-beta promoter. Cell, 2000. 103(4): p. 667-78.

59. Wathelet, M.G., et al., Virus infection induces the assembly of coordinately activated transcription factors on the IFN-beta enhancer in vivo. Molecular Cell, 1998. 1(4): p. 507-518.

60. Maniatis, T., et al., Structure and function of the interferon-beta enhanceosome. Cold Spring Harb Symp Quant Biol, 1998. 63: p. 609-20.

61. Zurita, M. and C. Merino, The transcriptional complexity of the TFIIH complex. Trends in Genetics, 2003. 19(10): p. 578-84.

62. Coin, F., J.C. Marinoni, and J.M. Egly, Mutations in XPD helicase prevent its interaction and regulation by $p 44$, another subunit of TFIIH, resulting in Xeroderma pigmentosum (XP) and trichothiodystrophy (TTD) phenotypes. Pathol Biol (Paris), 1998. 46(9): p. 679-80.

63. Keriel, A., et al., XPD mutations prevent TFIIH-dependent transactivation by nuclear receptors and phosphorylation of RARalpha. Cell, 2002. 109(1): p. 12535 .

64. Steinman, R.M., Dendritic cells in vivo: a key target for a new vaccine science. Immunity, 2008. 29(3): p. 319-24.

65. Alvarez, D., E.H. Vollmann, and U.H. von Andrian, Mechanisms and consequences of dendritic cell migration. Immunity, 2008. 29(3): p. 325-342.

66. Fogg, D.K., et al., A clonogenic bone marrow progenitor specific for macrophages and dendritic cells. Science, 2006. 311(5757): p. 83-87.

67. Fogg, D.K., A clonogenic bone marrow progenitor specific for macrophages and dendritic cells. Nature Reviews Immunology, 2006. 6(1): p. 8-8.

68. Villadangos, J.A. and L. Young, Antigen-presentation properties of plasmacytoid dendritic cells. Immunity, 2008. 29(3): p. 352-61.

69. Freer, G. and D. Matteucci, Influence of dendritic cells on viral pathogenicity. PLoS Pathog, 2009. 5(7): p. e1000384.

70. Kadowaki, N., et al., Natural interferon alpha/beta-producing cells link innate and adaptive immunity. Journal of Experimental Medicine, 2000. 192(2): p. 219225. 
71. Boonstra, A., et al., Flexibility of mouse classical and plasmacytoid-derived dendritic cells in directing Thelper type 1 and 2 cell development: Dependency on antigen dose and differential toll-like receptor ligation. Journal of Experimental Medicine, 2003. 197(1): p. 101-109.

72. Boule, M.W., et al., Toll-like receptor 9-dependent and-independent dendritic cell activation by chromatin-immunoglobulin $G$ complexes. Journal of Experimental Medicine, 2004. 199(12): p. 1631-1640.

73. Lande, R., et al., Plasmacytoid dendritic cells sense self-DNA coupled with antimicrobial peptide. Nature, 2007. 449(7162): p. 564-U6.

74. Di Pucchio, T., et al., Direct proteasome-independent cross-presentation of viral antigen by plasmacytoid dendritic cells on major histocompatibility complex class I. Nature Immunology, 2008. 9(5): p. 551-557.

75. Colonna, M., G. Trinchieri, and Y.J. Liu, Plasmacytoid dendritic cells in immunity. Nature Immunology, 2004. 5(12): p. 1219-1226.

76. Grouard, G., et al., The enigmatic plasmacytoid T cells develop into dendritic cells with interleukin (IL)-3 and CD40-ligand. Journal of Experimental Medicine, 1997. 185(6): p. 1101-1111.

77. Fiebiger, E., et al., Cytokines regulate proteolysis in major histocompatibility complex class II-dependent antigen presentation by dendritic cells. Journal of Experimental Medicine, 2001. 193(8): p. 881-892.

78. Asselin-Paturel, C., et al., Mouse type I IFN-producing cells are immature APCs with plasmacytoid morphology. Nature Immunology, 2001. 2(12): p. 1144-50.

79. Cella, M., et al., Plasmacytoid dendritic cells activated by influenza virus and CD40L drive a potent TH1 polarization. Nature Immunology, 2000. 1(4): p. 30510 .

80. Krug, A., et al., Interferon-producing cells fail to induce proliferation of naive $T$ cells but can promote expansion and $T$ helper 1 differentiation of antigenexperienced unpolarized T cells. Journal of Experimental Medicine, 2003. 197(7): p. 899-906.

81. Iezzi, G., D. Scheidegger, and A. Lanzavecchia, Migration and function of antigen-primed nonpolarized $T$ lymphocytes in vivo. Journal of Experimental Medicine, 2001. 193(8): p. 987-993.

82. Moseman, E.A., et al., Human plasmacytoid dendritic cells activated by CpG oligodeoxynucleotides induce the generation of $C D 4(+) C D 25(+)$ regulatory $T$ cells. Journal of Immunology, 2004. 173(7): p. 4433-4442.

83. Allenspach, E.J., et al., Migratory and Lymphoid-Resident Dendritic Cells Cooperate to Efficiently Prime Naive CD4 T cells. Immunity, 2008. 29(5): p. 795806.

84. Alexopoulou, L., et al., Recognition of double-stranded RNA and activation of NF-kappa B by Toll-like receptor 3. Nature, 2001. 413(6857): p. 732-738.

85. Kato, H., et al., Cell type-specific involvement of RIG-I in antiviral response. Immunity, 2005. 23(1): p. 19-28.

86. Pichlmair, A., et al., RIG-I-mediated antiviral responses to single-stranded RNA bearing 5 '-phosphates. Science, 2006. 314(5801): p. 997-1001. 
87. Ingulli, E., et al., In vivo detection of dendritic cell antigen presentation to CD4(+) T cells. Journal of Experimental Medicine, 1997. 185(12): p. 2133-41.

88. Mclellan, A.D., et al., Activation of Human Peripheral-Blood Dendritic Cells Induces the Cd86 Costimulatory Molecule. European Journal of Immunology, 1995. 25(7): p. 2064-2068.

89. McLellan, A.D., et al., Human dendritic cells activate T lymphocytes via a CD40: CD40 ligand-dependent pathway. Eur J Immunol, 1996. 26(6): p. 1204-10.

90. Cella, M., et al., Inflammatory stimuli induce accumulation of MHC class II complexes on dendritic cells. Nature, 1997. 388(6644): p. 782-7.

91. Pierre, P., et al., Developmental regulation of MHC class II transport in mouse dendritic cells. Nature, 1997. 388(6644): p. 787-92.

92. Schoenberger, S.P., et al., T-cell help for cytotoxic T lymphocytes is mediated by CD40-CD40L interactions. Nature, 1998. 393(6684): p. 480-483.

93. Bennett, S.R.M., et al., Help for cytotoxic-T-cell responses is mediated by CD40 signalling. Nature, 1998. 393(6684): p. 478-480.

94. Ridge, J.P., F. Di Rosa, and P. Matzinger, A conditioned dendritic cell can be a temporal bridge between a CD4(+) T-helper and a T-killer cell. Nature, 1998. 393(6684): p. 474-478.

95. Knight, S.C., B.A. Askonas, and S.E. Macatonia, Dendritic cells as targets for cytotoxic $T$ lymphocytes. Dendritic Cells in Fundamental and Clinical Immunology, Vol 3, 1997. 417: p. 389-394.

96. Knight, S.C., W. Elsley, and H. Wang, Mechanisms of loss of functional dendritic cells in HIV-1 infection. Journal of Leukocyte Biology, 1997. 62(1): p. 78-81.

97. Morgan, M.M., C.C. Clayton, and M.M. Heinricher, Dissociation of hyperalgesia from fever following intracerebroventricular administration of interleukin-1beta in the rat. Brain Res, 2004. 1022(1-2): p. 96-100.

98. Kakumu, S., et al., Localisation of intrahepatic interleukin 6 in patients with acute and chronic liver disease. J Clin Pathol, 1992. 45(5): p. 408-11.

99. Hirano, T., K. Ishihara, and M. Hibi, Roles of STAT3 in mediating the cell growth, differentiation and survival signals relayed through the IL-6 family of cytokine receptors. Oncogene, 2000. 19(21): p. 2548-56.

100. Vlahopoulos, S., et al., Nuclear factor-kappaB-dependent induction of interleukin8 gene expression by tumor necrosis factor alpha: evidence for an antioxidant sensitive activating pathway distinct from nuclear translocation. Blood, 1999. 94(6): p. 1878-89.

101. Kalinski, P., et al., IL-12-deficient dendritic cells, generated in the presence of prostaglandin E2, promote type 2 cytokine production in maturing human naive $T$ helper cells. J Immunol, 1997. 159(1): p. 28-35.

102. Promega, C., $p R L-S V 40$ Vector.

103. Laughlin, L.W., et al., Epidemic Rift-Valley Fever in Egypt-Observations of the Spectrum of Human Illness. Transactions of the Royal Society of Tropical Medicine and Hygiene, 1979. 73(6): p. 630-633.

104. Weidmann, M., et al., Rapid detection of important human pathogenic Phleboviruses. Journal of Clinical Virology, 2008. 41(2): p. 138-142. 
105. Sallusto, F. and A. Lanzavecchia, Efficient presentation of soluble antigen by cultured human dendritic cells is maintained by granulocyte/macrophage colonystimulating factor plus interleukin 4 and downregulated by tumor necrosis factor alpha. J Exp Med, 1994. 179(4): p. 1109-18.

106. Chou, Y.C., et al., Evaluation of transcriptional efficiency of hepatitis B virus covalently closed circular DNA by reverse transcriptiori-PCR combined with the restriction enzyme digestion method. Journal of Virology, 2005. 79(3): p. 18131823.

107. Kuo, T.M., et al., HBV replication is significantly reduced by IL-6. Journal of Biomedical Science, 2009. 16: p. - .

108. Jego, G., et al., Plasmacytoid dendritic cells induce plasma cell differentiation through type I interferon and interleukin 6. Immunity, 2003. 19(2): p. 225-34.

109. Gowen, B.B., et al., TLR3 is essential for the induction of protective immunity against Punta Toro virus infection by the double-stranded RNA (dsRNA), poly(I : C12U), but not poly $(I: C)$ : Differential, recognition of synthetic dsRNA molecules. Journal of Immunology, 2007. 178(8): p. 5200-5208.

110. Sen, G.C., Viruses and interferons. Annual Review of Microbiology, 2001. 55: p. 255-281.

111. Sall, A.A., et al., Single-tube and nested reverse transcriptase-polymerase chain reaction for detection of Rift Valley fever virus in human and animal sera. J Virol Methods, 2001. 91(1): p. 85-92.

112. Bouloy, M., et al., Genetic evidence for an interferon-antagonistic function of rift valley fever virus nonstructural protein NSs. J Virol, 2001. 75(3): p. 1371-7.

113. Le May, N., et al., TFIIH transcription factor, a target for the Rift Valley hemorrhagic fever virus. Cell, 2004. 116(4): p. 541-50.

114. Muller, R., et al., Characterization of clone 13, a naturally attenuated avirulent isolate of Rift Valley fever virus, which is altered in the small segment. American Journal of Tropical Medicine and Hygiene, 1995. 53(4): p. 405-11.

115. Vialat, P., et al., Mapping of the mutations present in the genome of the Rift Valley fever virus attenuated MP12 strain and their putative role in attenuation. Virus Research, 1997. 52(1): p. 43-50.

116. Klagge, I.M. and S. Schneider-Schaulies, Virus interactions with dendritic cells. Journal of General Virology, 1999. 80 ( Pt 4): p. 823-33.

117. Kittan, N.A., et al., Impaired plasmacytoid dendritic cell innate immune responses in patients with herpes virus-associated acute retinal necrosis. Journal of Immunology, 2007. 179(6): p. 4219-4230.

118. Poeck, H., et al., Plasmacytoid dendritic cells, antigen, and CpG-C license human $B$ cells for plasma cell differentiation and immunoglobulin production in the absence of T-cell help. Blood, 2004. 103(8): p. 3058-64.

119. Gowen, B.B., et al., TLR3 deletion limits mortality and disease severity due to Phlebovirus infection. J Immunol, 2006. 177(9): p. 6301-7.

120. Damas, P., et al., Cytokine serum level during severe sepsis in human IL-6 as a marker of severity. Ann Surg, 1992. 215(4): p. 356-62.

121. Spiegel, M., et al., Interaction of severe acute respiratory syndrome-associated coronavirus with dendritic cells. J Gen Virol, 2006. 87(Pt 7): p. 1953-60. 
122. Lockridge, K.M., et al., Primate cytomegaloviruses encode and express an IL-10like protein. Virology, 2000. 268(2): p. 272-280.

123. Krug, A., et al., IFN-producing cells respond to CXCR3 ligands in the presence of CXCL12 and secrete inflammatory chemokines upon activation. J Immunol, 2002. 169(11): p. 6079-83.

124. Penna, G., et al., Cutting edge: differential chemokine production by myeloid and plasmacytoid dendritic cells. J Immunol, 2002. 169(12): p. 6673-6.

125. Megjugorac, N.J., et al., Virally stimulated plasmacytoid dendritic cells produce chemokines and induce migration of T and NK cells. J Leukoc Biol, 2004. 75(3): p. 504-14.

126. Jego, G., et al., Plasmacytoid dendritic cells induce plasma cell differentiation through type I interferon and interleukin 6. Immunity, 2003. 19(2): p. 225-234.

127. Hegde, S., J. Pahne, and S. Smola-Hess, Novel immunosuppressive properties of interleukin-6 in dendritic cells: inhibition of NF-kappaB binding activity and CCR7 expression. Faseb Journal, 2004. 18(12): p. 1439-41.

128. Colonna, M., A. Krug, and M. Cella, Interferon-producing cells: on the front line in immune responses against pathogens. Current Opinion in Immunology, 2002. 14(3): p. 373-9.

129. Heil, F., et al., Species-specific recognition of single-stranded RNA via toll-like receptor 7 and 8. Science, 2004. 303(5663): p. 1526-1529.

130. Higashihara, M., H. Koyama, and Y. Igarashi, Induction of virus-inhibiting factor or interferon in mice by strains with different virulence of Rift Valley fever virus. Kitasato Arch Exp Med, 1972. 45(1): p. 33-43.

131. Yoneyama, M., et al., Direct triggering of the type I interferon system by virus infection: activation of a transcription factor complex containing IRF-3 and CBP/p300. Embo Journal, 1998. 17(4): p. 1087-95.

132. Alcami, A. and U.H. Koszinowski, Viral mechanisms of immune evasion. Trends Microbiol, 2000. 8(9): p. 410-8.

133. Beck, K., et al., Human cytomegalovirus impairs dendritic cell function: a novel mechanism of human cytomegalovirus immune escape. Eur J Immunol, 2003. 33(6): p. 1528-38.

134. Weber, F., G. Kochs, and O. Haller, Inverse interference: how viruses fight the interferon system. Viral Immunol, 2004. 17(4): p. 498-515.

135. Pichyangkul, S., et al., A blunted blood plasmacytoid dendritic cell response to an acute systemic viral infection is associated with increased disease severity. Journal of Immunology, 2003. 171(10): p. 5571-5578.

136. Wallich, R., et al., Gene structure, promoter characterization, and basis for alternative mRNA splicing of the human CD58 gene. Journal of Immunology, 1998. 160(6): p. 2862-2871.

137. Wang, J., et al., Structure of a heterophilic adhesion complex between the human CD2 and CD58 (LFA-3) counterreceptors. Cell, 1999. 97(6): p. 791-803.

138. Ruddle, N.H. and B.H. Waksman, Cytotoxicity mediated by soluble antigen and lymphocytes in delayed hypersensitivity. 3. Analysis of mechanism. Journal of Experimental Medicine, 1968. 128(6): p. 1267-79. 
139. Lechmann, M., et al., CD83 on dendritic cells: more than just a marker for maturation. Trends in Immunology, 2002. 23(6): p. 273-275.

140. Law, H.K., et al., Chemokine up-regulation in SARS-coronavirus-infected, monocyte-derived human dendritic cells. Blood, 2005. 106(7): p. 2366-74.

141. Ziegler, T., et al., Severe acute respiratory syndrome coronavirus fails to activate cytokine-mediated innate immune responses in cultured human monocyte-derived dendritic cells. J Virol, 2005. 79(21): p. 13800-5.

142. Chehimi, J., et al., Dendritic Cells and Ifn-Alpha-Producing Cells Are 2 Functionally Distinct Non-B, Non-Monocytic Hla-Dr+ Cell Subsets in Human Peripheral-Blood. Immunology, 1989. 68(4): p. 486-490.

143. Fonteneau, J.F., et al., Activation of influenza virus-specific CD4+ and CD8+T cells: a new role for plasmacytoid dendritic cells in adaptive immunity. Blood, 2003. 101(9): p. 3520-6.

144. Wang, Z., et al., Activation of CXCR4 triggers ubiquitination and down-regulation of major histocompatibility complex class I (MHC-I) on epithelioid carcinoma HeLa cells. Journal of Biological Chemistry, 2008. 283(7): p. 3951-9.

145. Billecocq, A., et al., NSs Protein of Rift Valley Fever Virus Blocks Interferon Production

by Inhibiting Host Gene Transcription. Journal of Virology, 2004. 78(18): p. 9798-9806. 


\section{$7 \quad$ Appendix}

\subsection{Additional results of proinflammatory cytokine production in mDCs infected by RVFV (chapter 3.6.1)}

Figures 28 to 35 show the expression of proinflammatory cytokines in mDCs infected with wtRFV ZH548 (red bar), with UV inactivated wtRVFV ZH548 (green bar), RVFV clone 13 (purple bar), UV inactivated RVFV clone 13 (blue bar), and mock control (dark blue bar) at 12h, 24h, 48h, and 72h (clockwise).
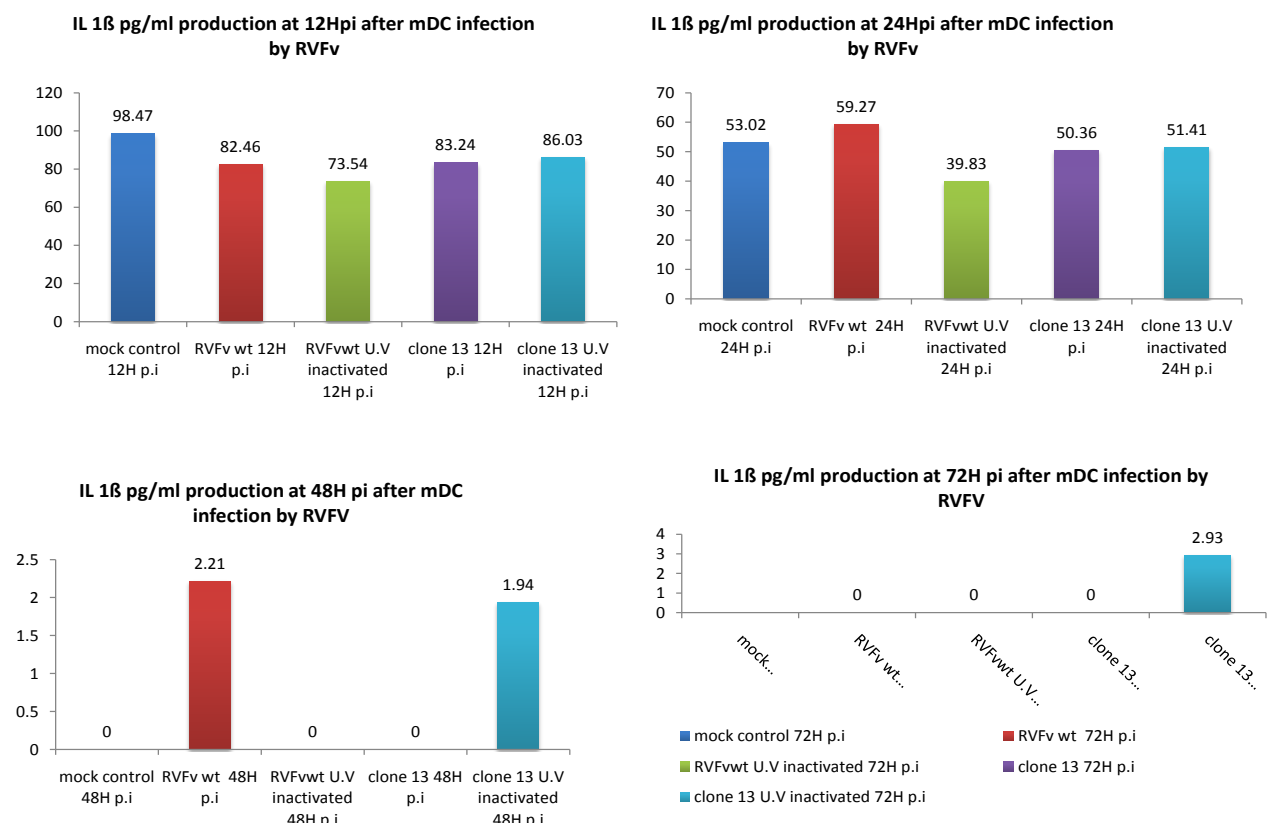

Figure 23. IL-1 $\beta$ production of human mDCs after infection with RVFV. 

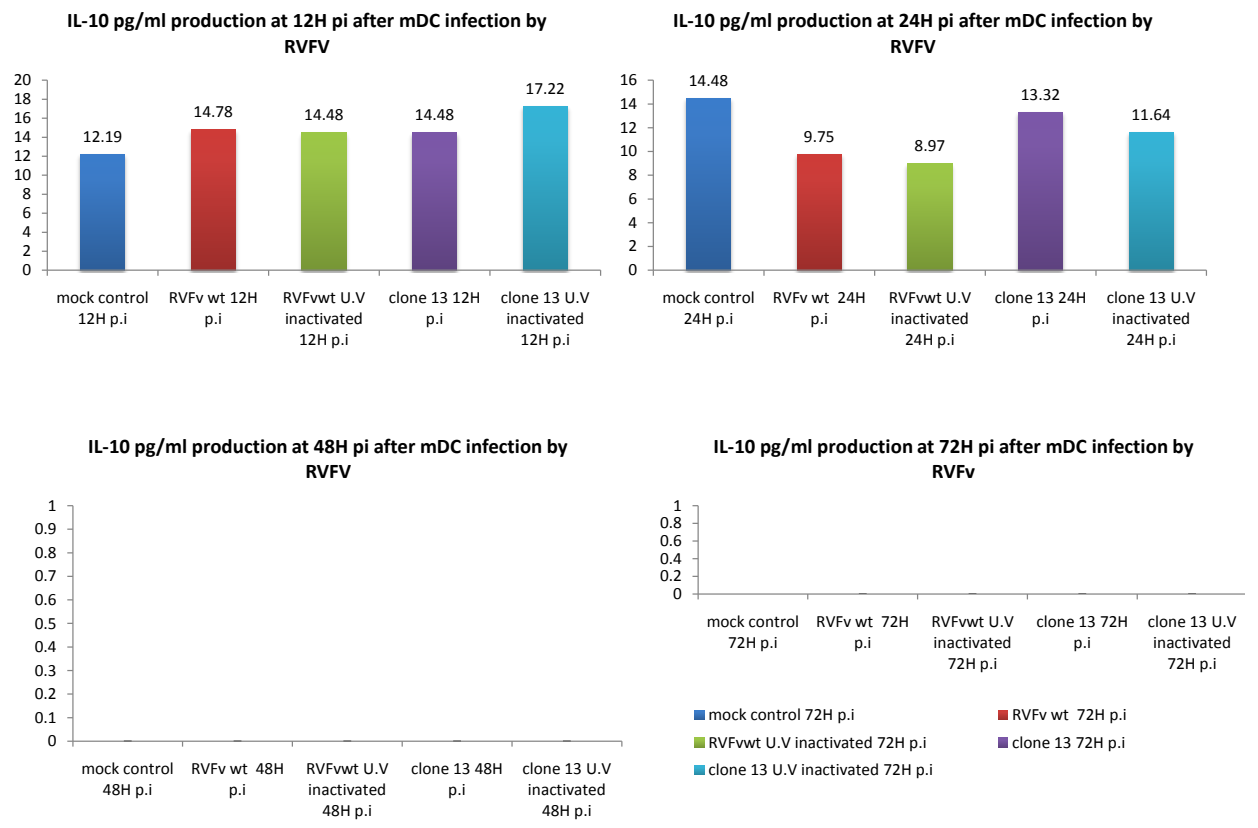

Figure 24. IL-10 production of human mDCs after infection with RVFV.
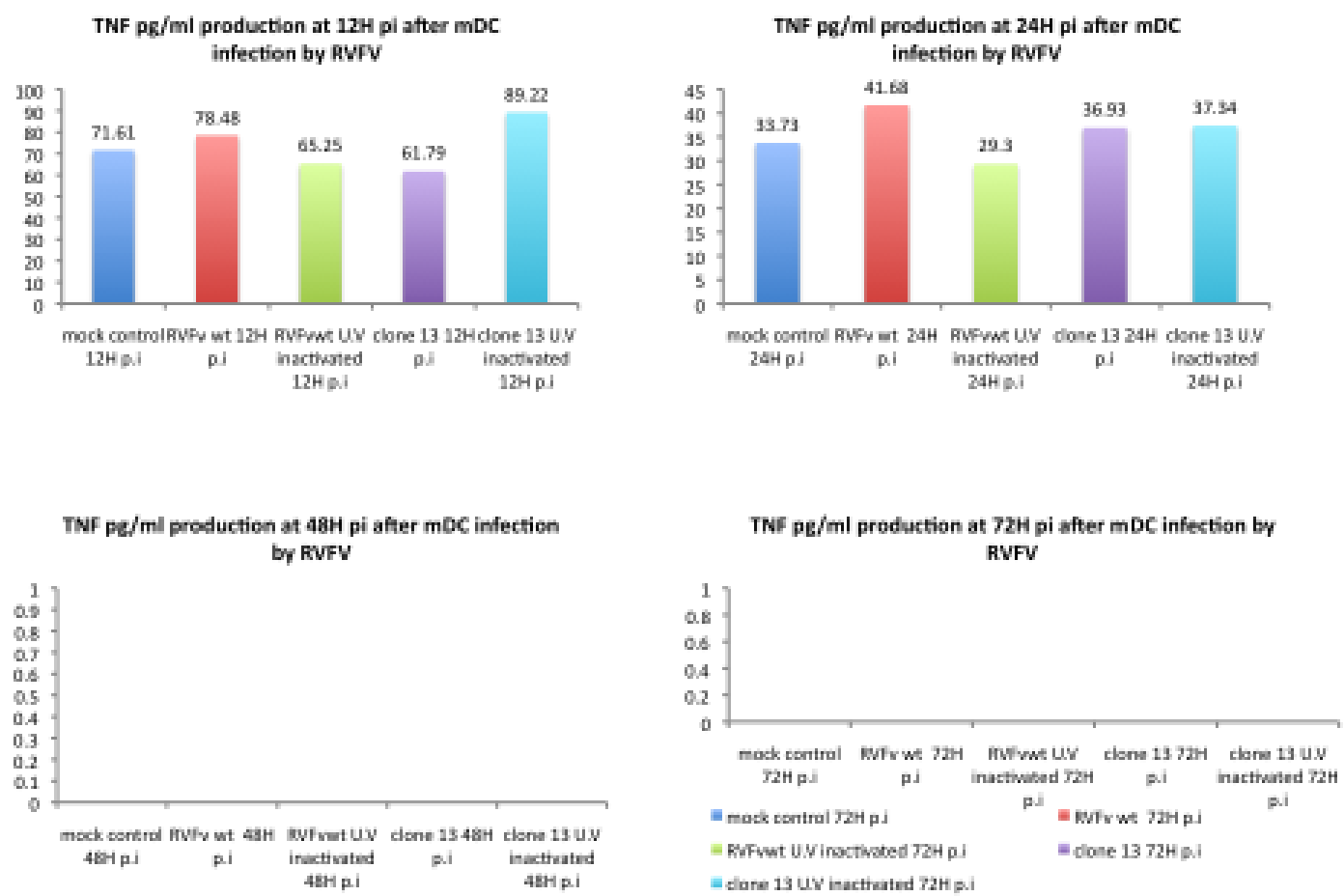

Figure 25. TNF $\alpha$ production of human mDCs after infection with RVFV. 

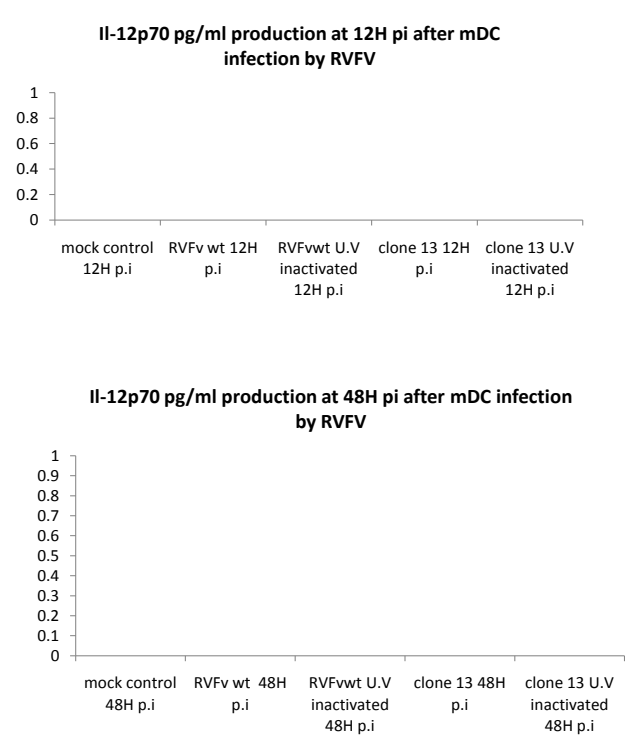
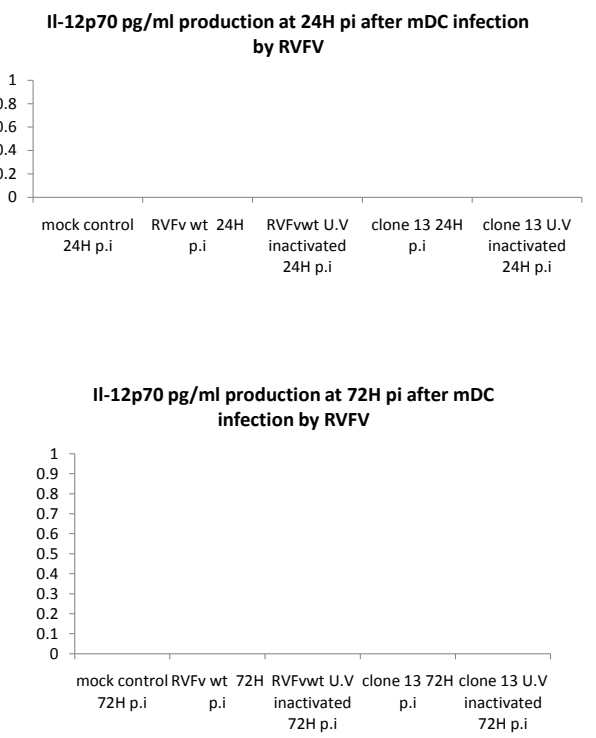

Figure 26. IL-12p70 alpha production of human mDCs after infection with RVFV.

\subsection{Additional results of proinflammatory cytokine production in mDCs infected by RVFV (chapter 3.6.1)}

Figures 28 to 35 show the expression of proinflammatory cytokines in mDCs infected with wtRFV ZH548 (red bar), with UV inactivated wtRVFV ZH548 (green bar), RVFV clone 13 (purple bar), UV inactivated RVFV clone 13 (blue bar), and mock control (dark blue bar) at 12h, 24h, 48h, and 72h (clockwise).
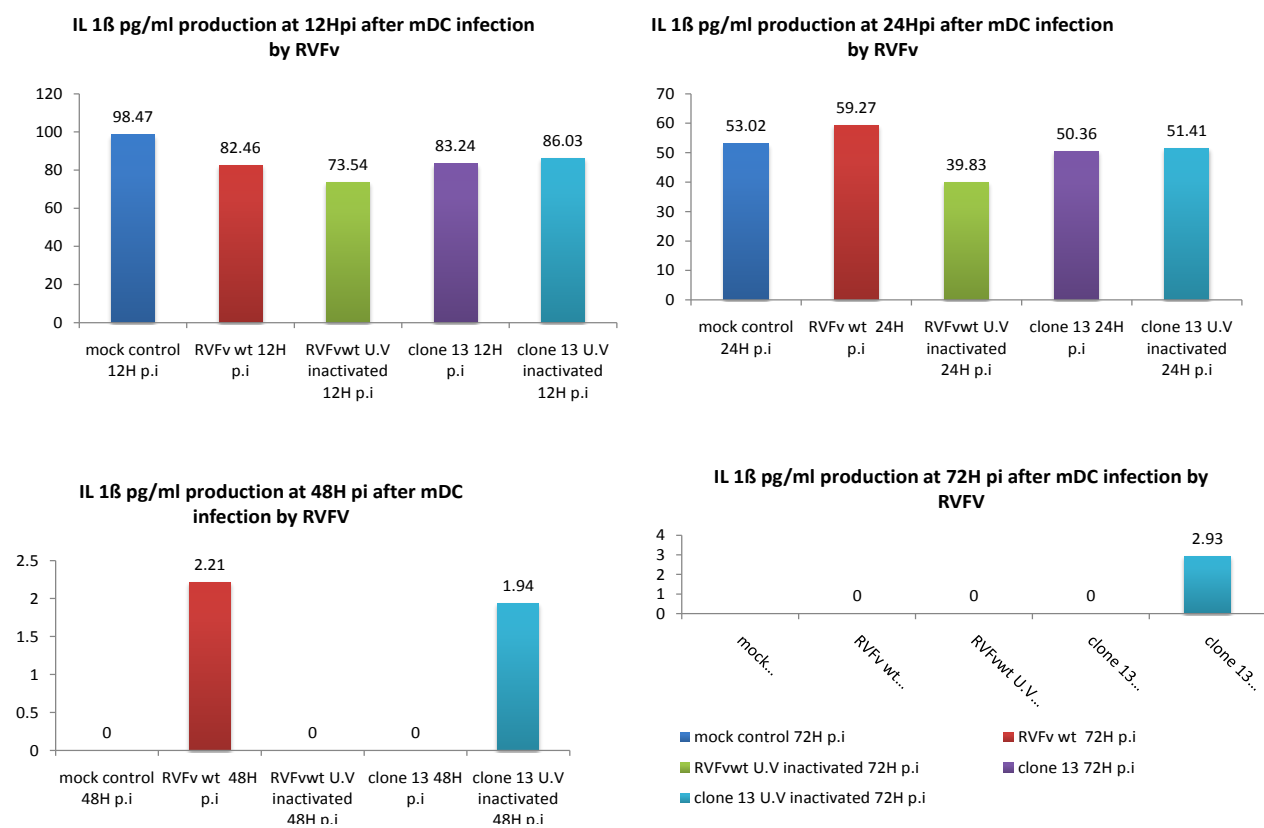

Figure 27. IL-1 $\beta$ production of human mDCs after infection with RVFV. 

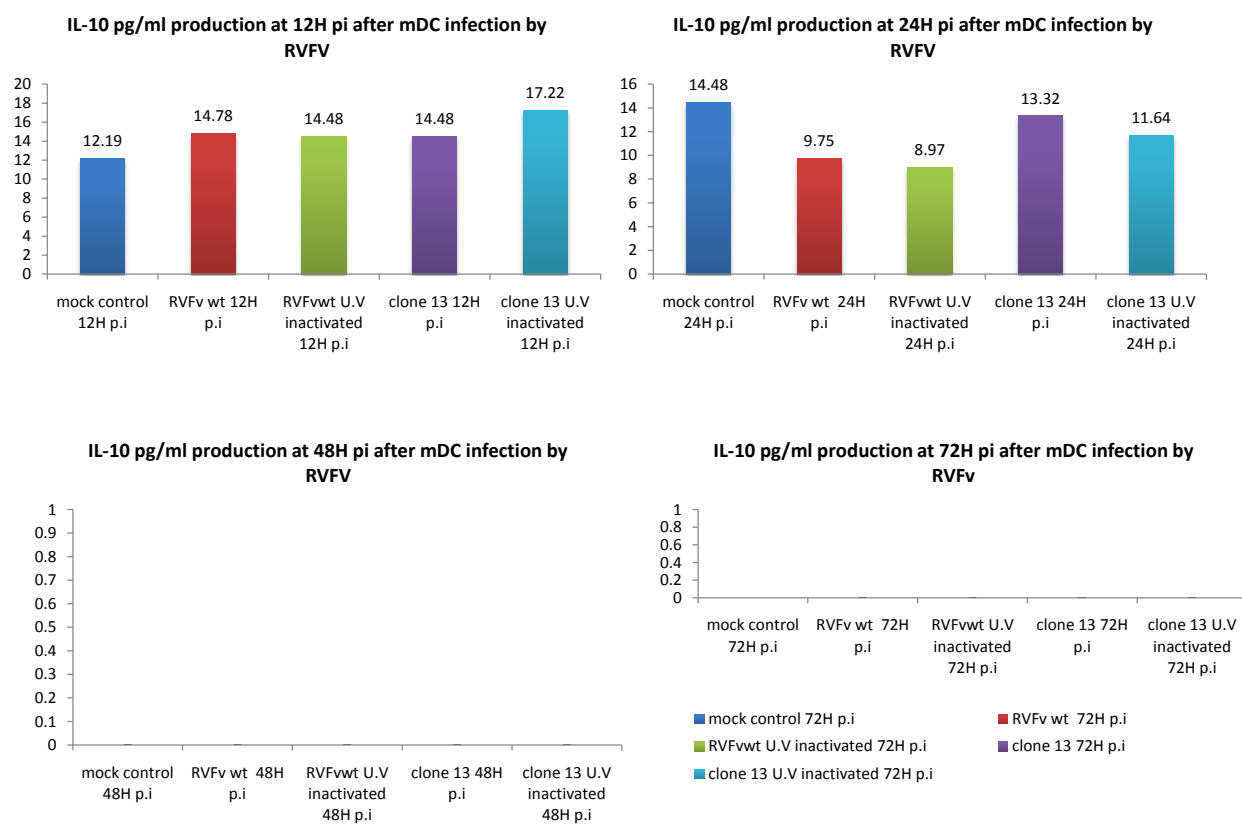

Figure 28. IL-10 production of human mDCs after infection with RVFV.
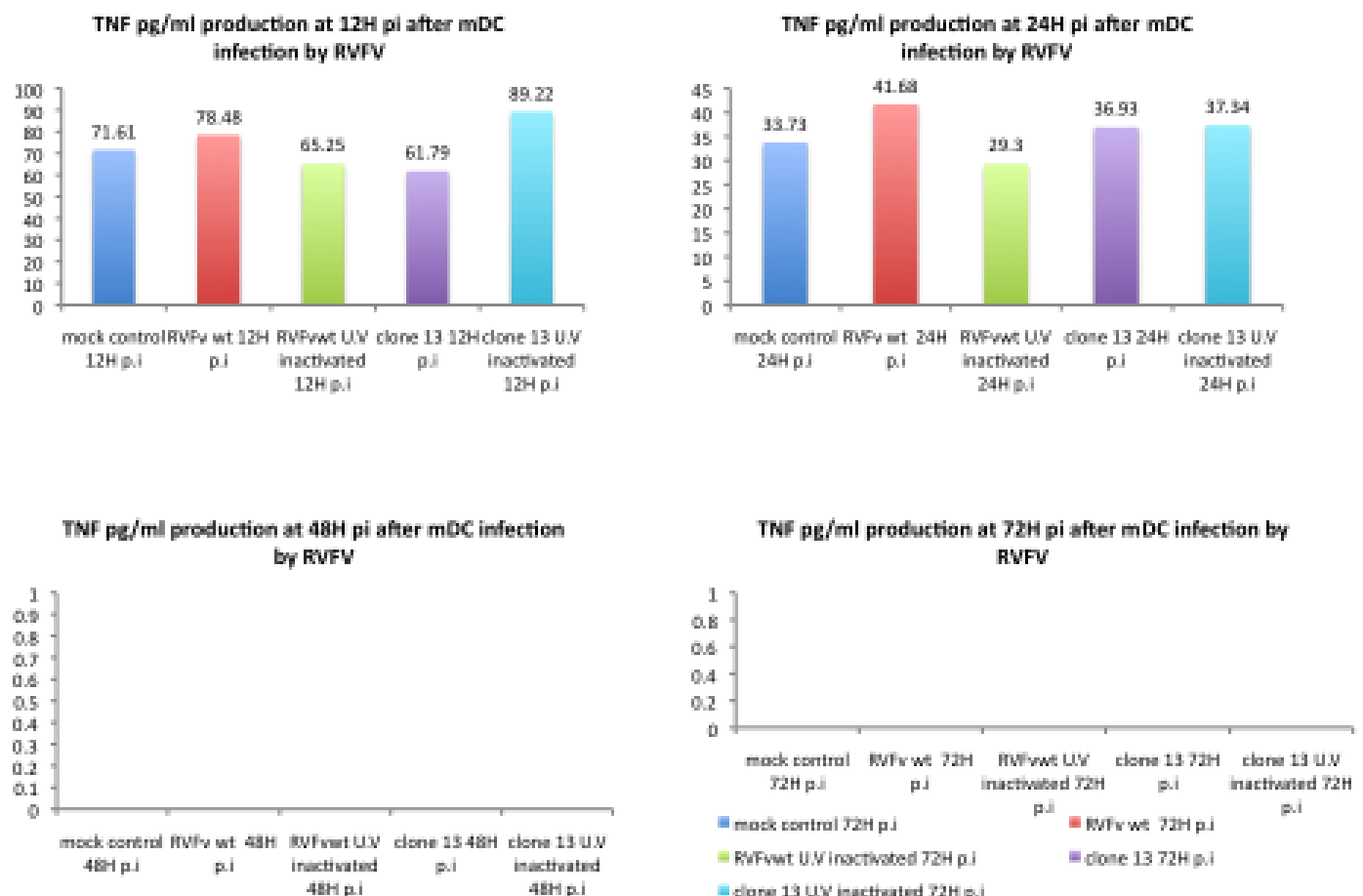

Figure 29. TNF $\alpha$ production of human mDCs after infection with RVFV. 

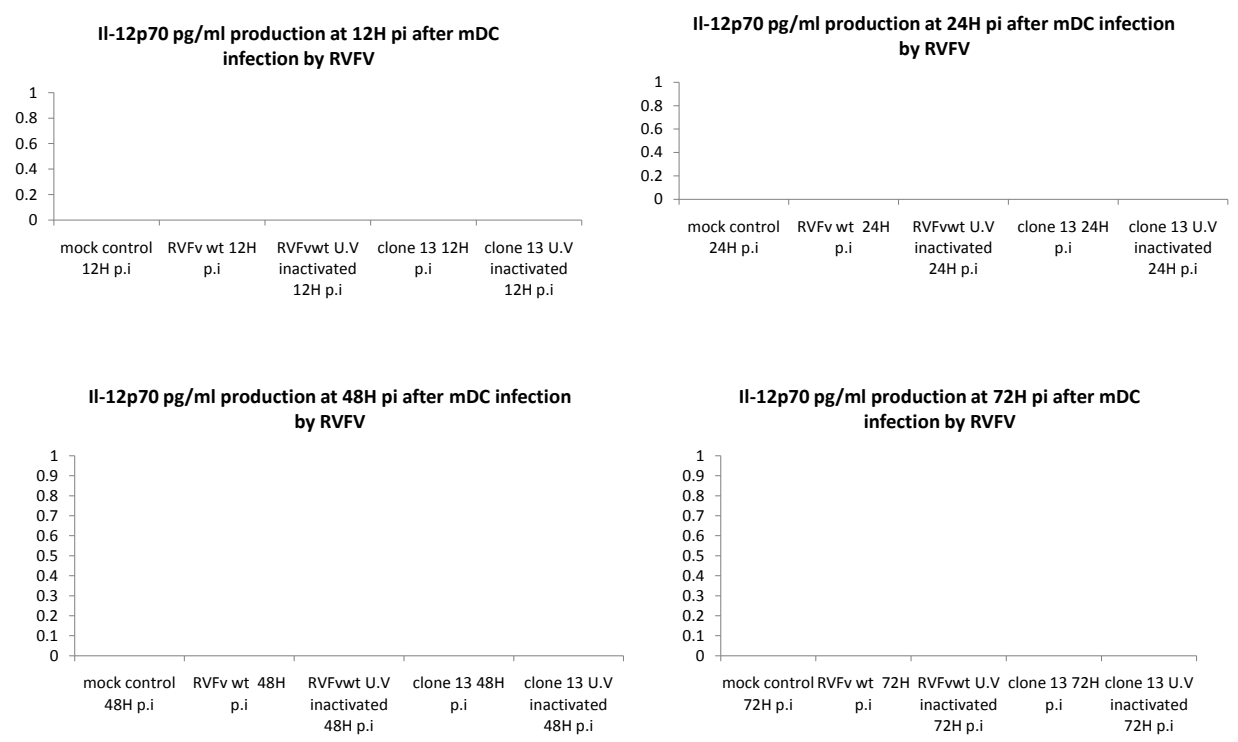

Figure 30. IL-12p70 alpha production of human mDCs after infection with RVFV.

\subsection{Additional results of proinflammatory cytokine production in pDCs infected by RVFV (chapter 3.6.2)}

Figures 36 to 40 show the expression of proinflammatory cytokines in pDCs infected with wtRFV ZH548 (red bar), with UV inactivated wtRVFV ZH548 (green bar), RVFV clone 13 (purple bar), UV inactivated RVFV clone 13 (light blue bar), and mock control (dark blue bar) at $12 \mathrm{~h}, 24 \mathrm{~h}, 48 \mathrm{~h}$, and $72 \mathrm{~h}$ (clockwise).
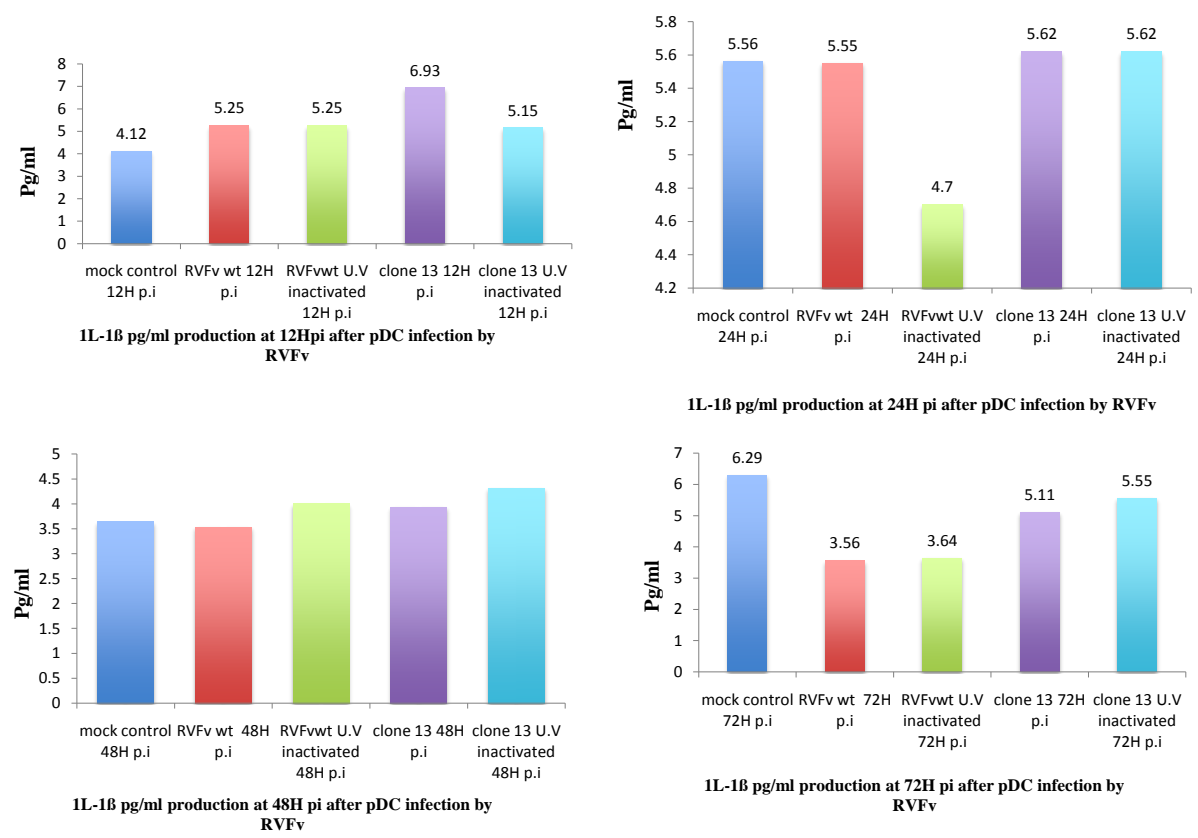

Figure 31. IL-1 $\beta$ production production of human pDCs after infection with RVFV at $12 \mathrm{~h}, 24 \mathrm{~h}, 48 \mathrm{~h}$, and $72 \mathrm{~h}$ (clockwise). 

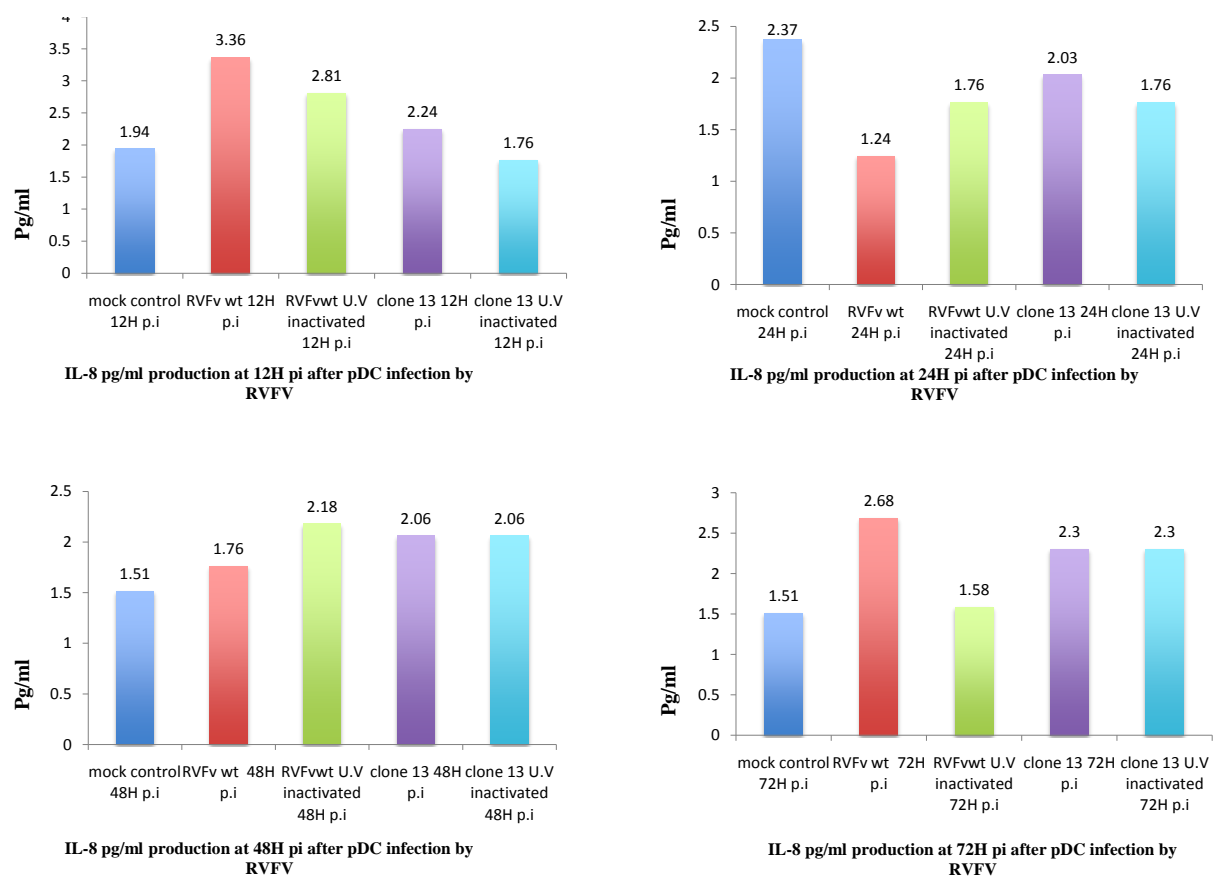

Figure 32. IL-8 production production of human pDCs after infection with RVFV at $12 \mathrm{~h}, 24 \mathrm{~h}, 48 \mathrm{~h}$, and $72 \mathrm{~h}$ (clockwise).

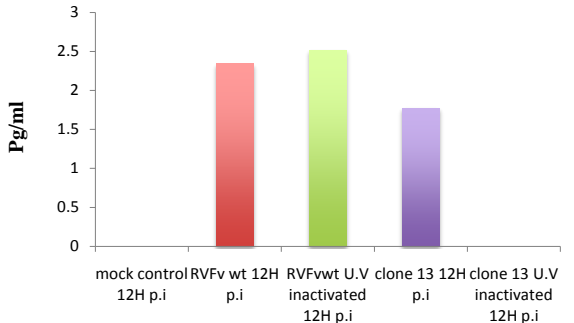

IL-10 pg/ml production at $12 \mathrm{H}$ pi after pDC infection by

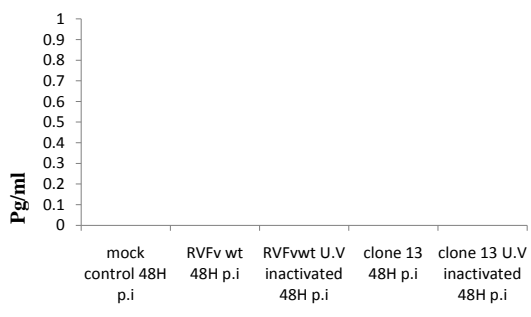

IL-10 pg/ml production at $48 \mathrm{H}$ pi after pDC infection by
RVFV

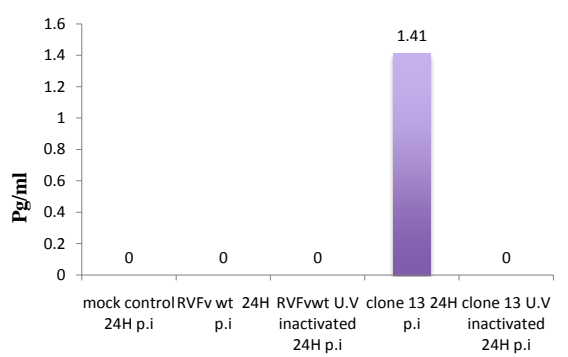

IL-10 $\mathrm{pg} / \mathrm{ml}$ production at $24 \mathrm{H}$ pi after pDC infection by

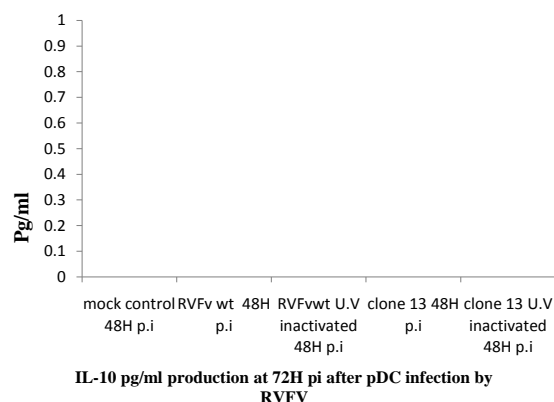

RVFV

Figure 33. IL-10 production production of human pDCs after infection with RVFV at $12 \mathrm{~h}, 24 \mathrm{~h}, 48 \mathrm{~h}$, and $72 \mathrm{~h}$ (clockwise). 

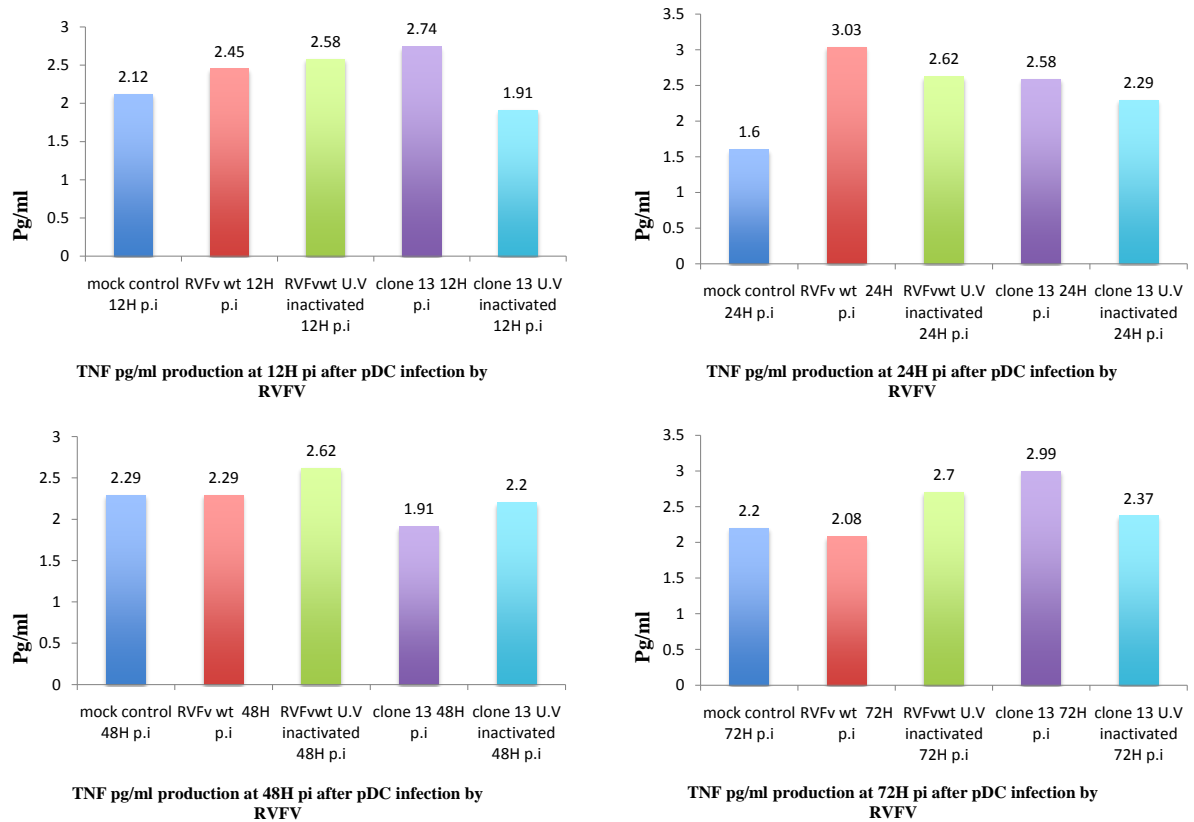

Figure 34. TNF $\alpha$ production of human pDCs after infection with RVFV at $12 \mathrm{~h}, 24 \mathrm{~h}, 48 \mathrm{~h}$, and $72 \mathrm{~h}$ (clockwise).
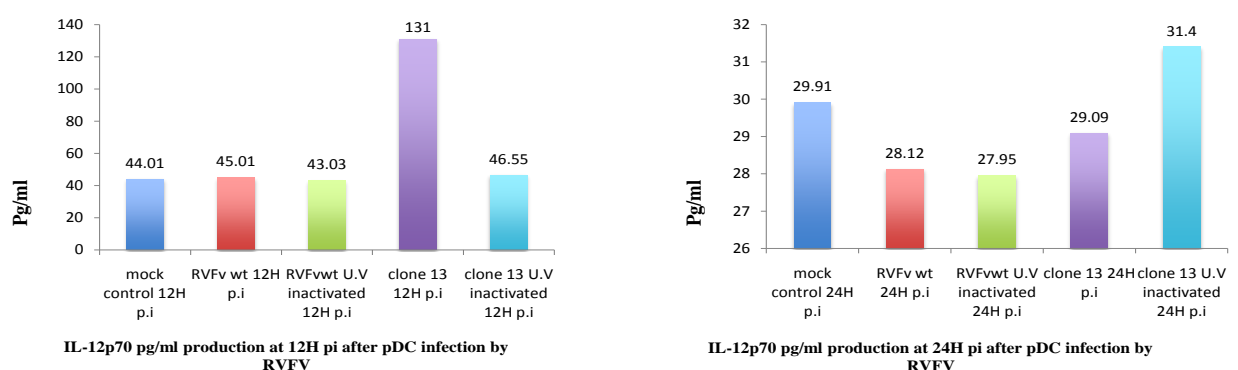

IL-12p70 pg/ml production at $24 \mathrm{H}$ pi after pDC infection by
RVFV
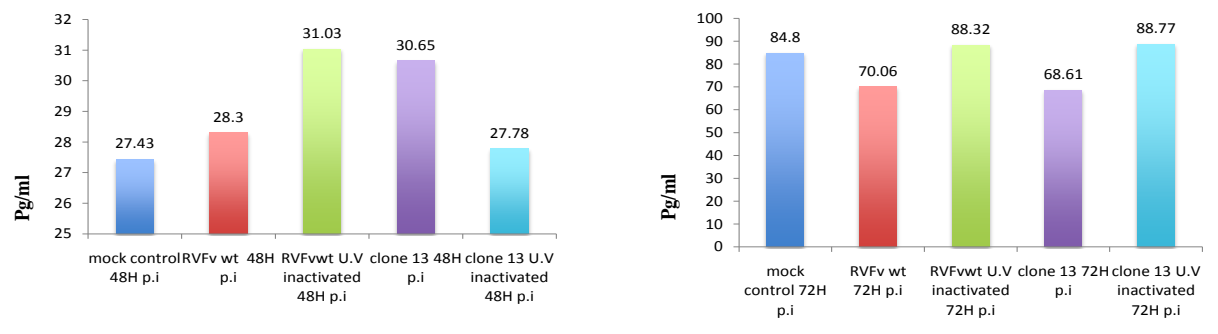

RVFV

Figure 35. IL-12p70 production of human pDCs after infection with RVFV at $12 \mathrm{~h}, 24 \mathrm{~h}, 48 \mathrm{~h}$, and $72 \mathrm{~h}$ (clockwise). 
7.4 Additional results for expression and regulation of surface marker molecules in mDCs infected by RVFV (Chapter 3.10)

In all figures results are indicated as follows: infection with wtRVFV (light blue), UV inactivated wtRVFV (purple), RVFV clone 13 (dark blue), UV inactivated RVFV clone 13 (red), and mock control (orange) at 12h, 24h, 48h, and 72h (clockwise).
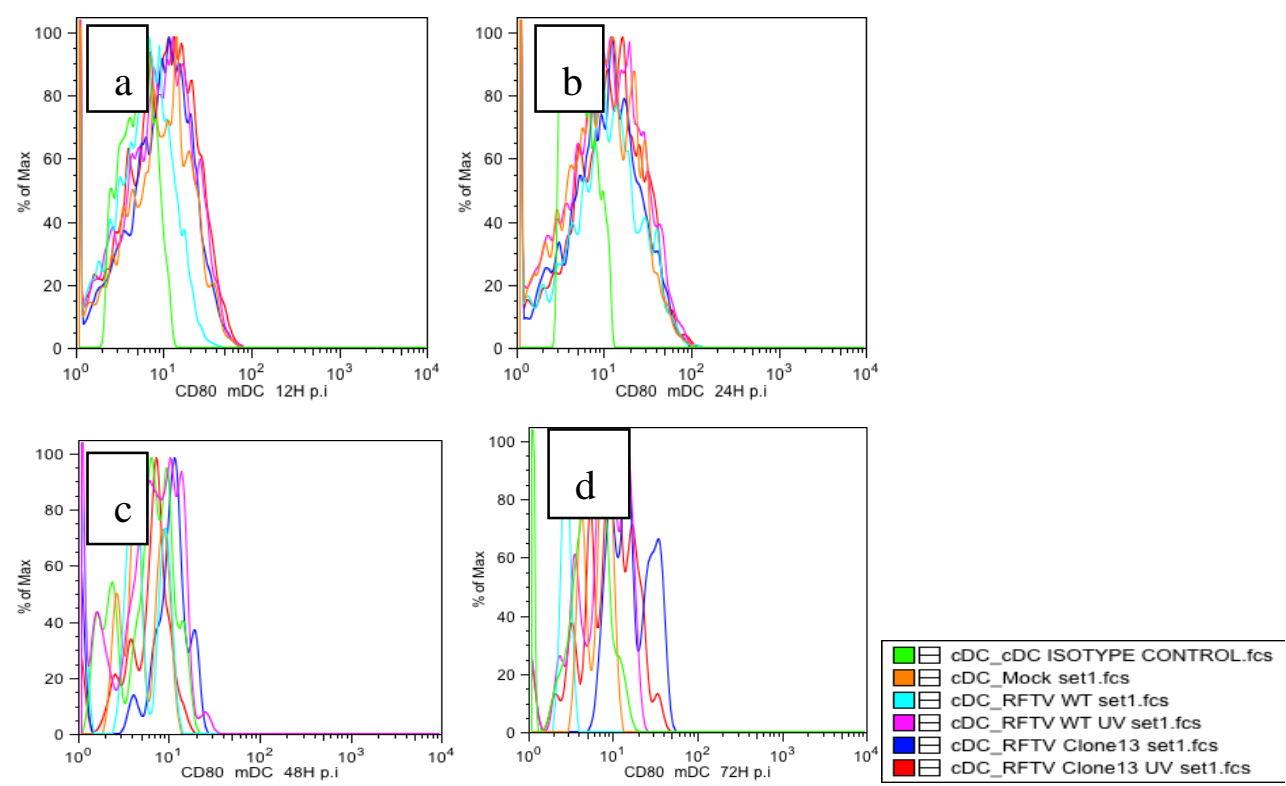

Figure 36. CD80 regulation after an infection of mDCs by RVFV.
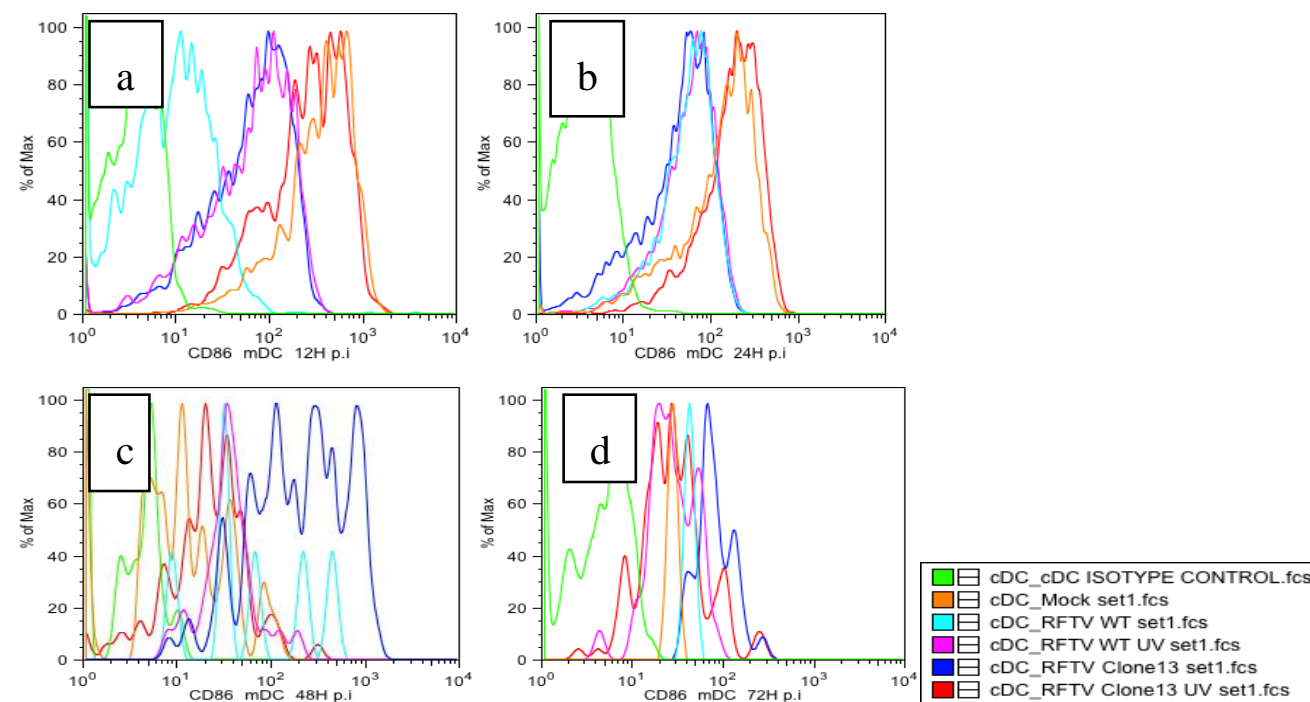

Figure 37. CD86 regulation after an infection of mDCs by RVFV. 

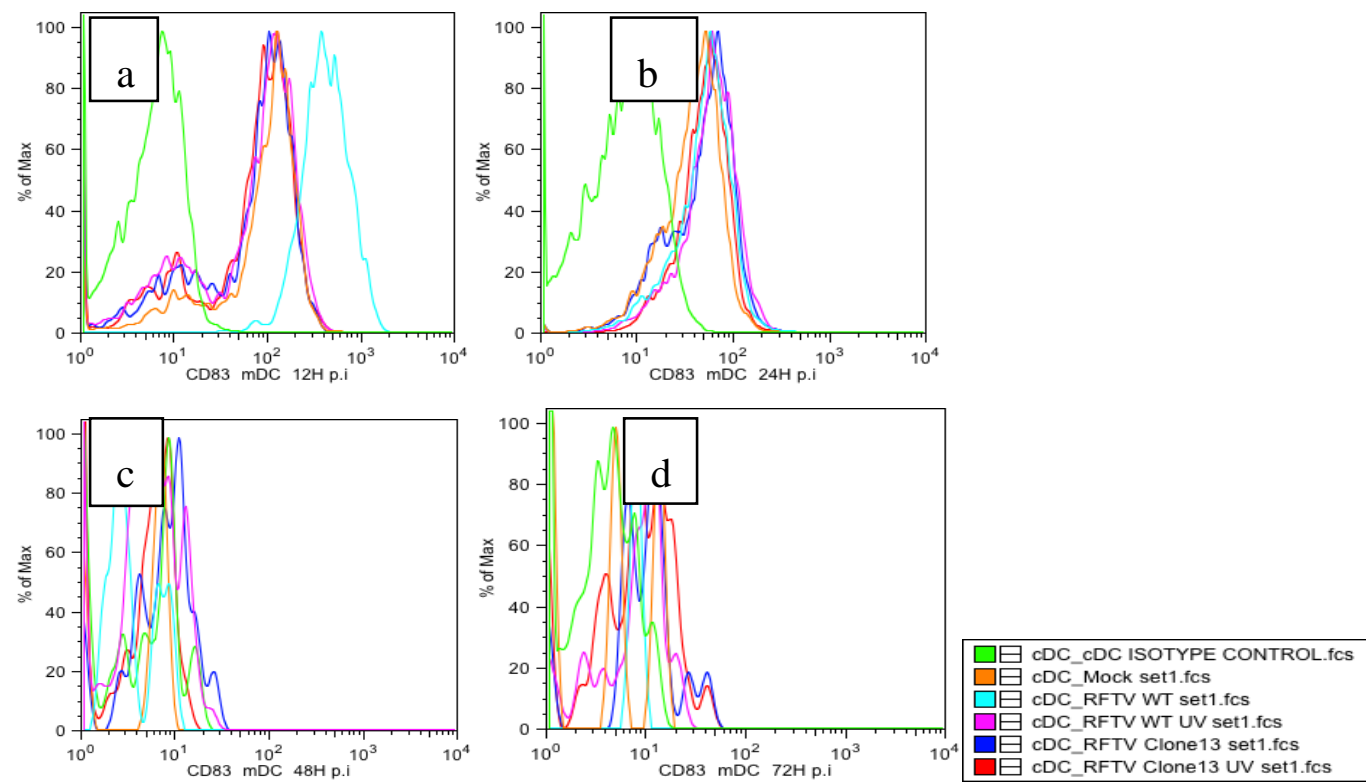

Figure 38. CD83 regulation after an infection of mDCs by RVFV.
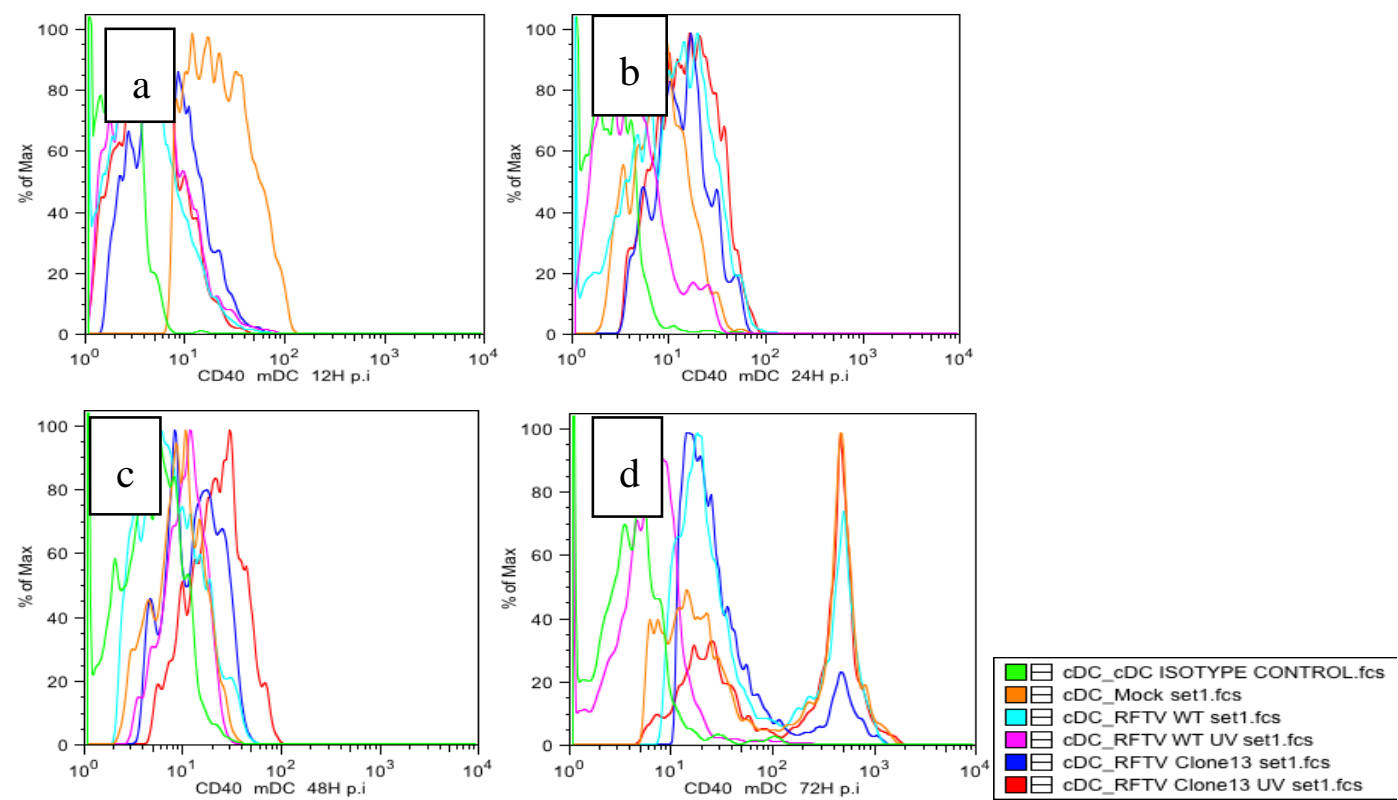

Figure 39. CD40 regulation after an infection of mDCs by RVFV. 

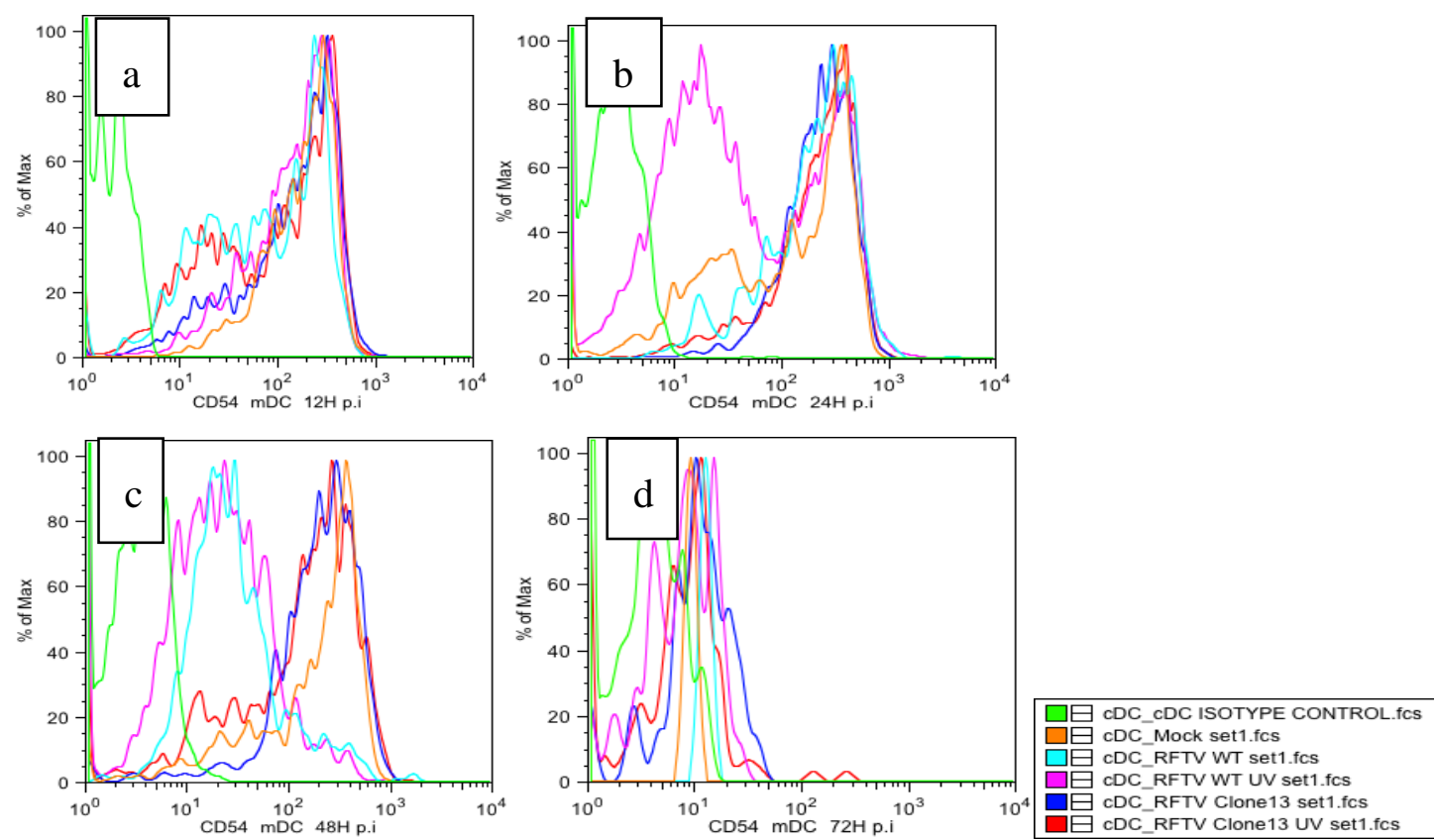

Figure 40. CD54 regulation after an infection of mDCs by RVFV.
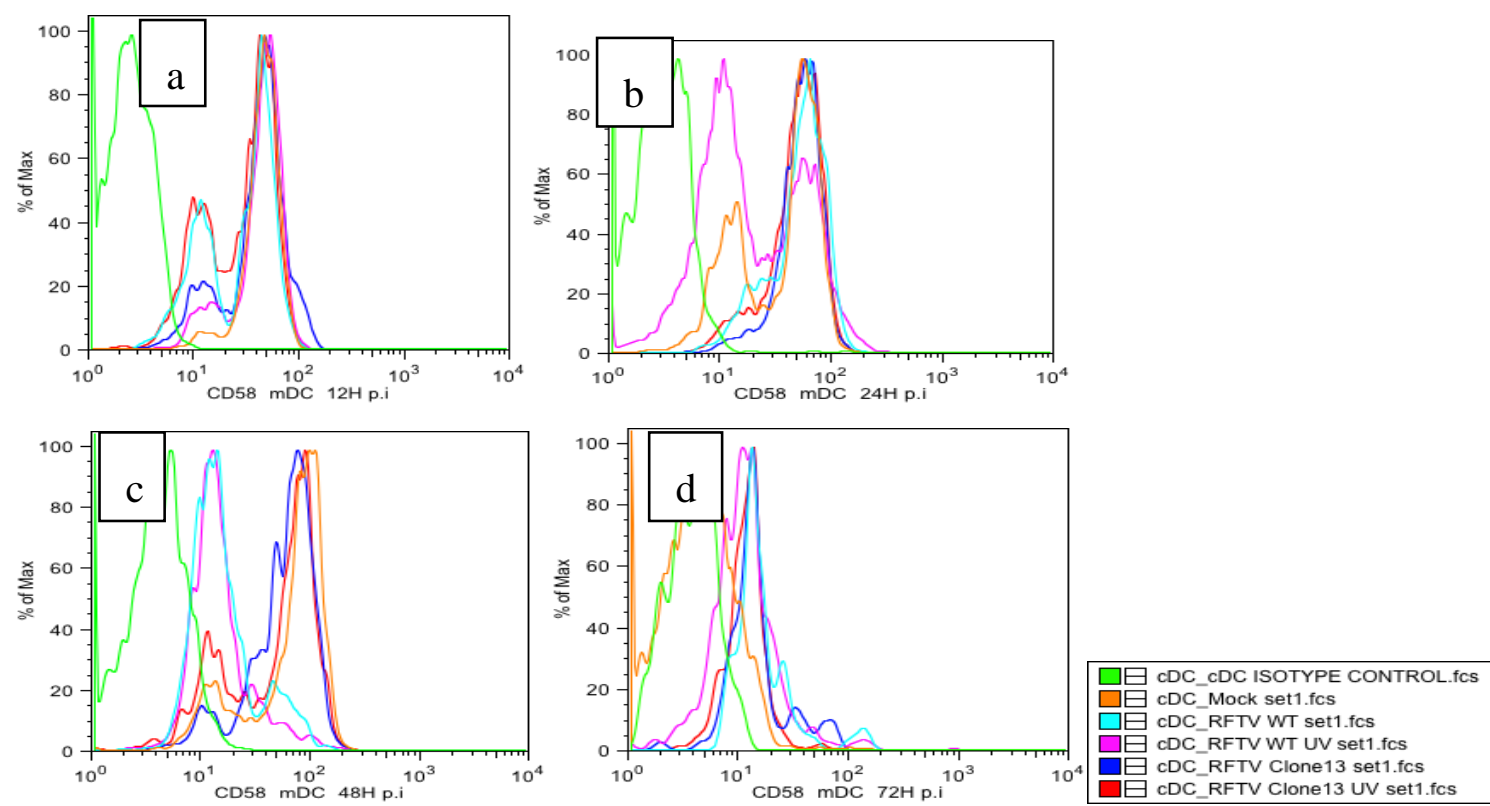

Figure 41. CD58 regulation after an infection of mDCs by RVFV. 

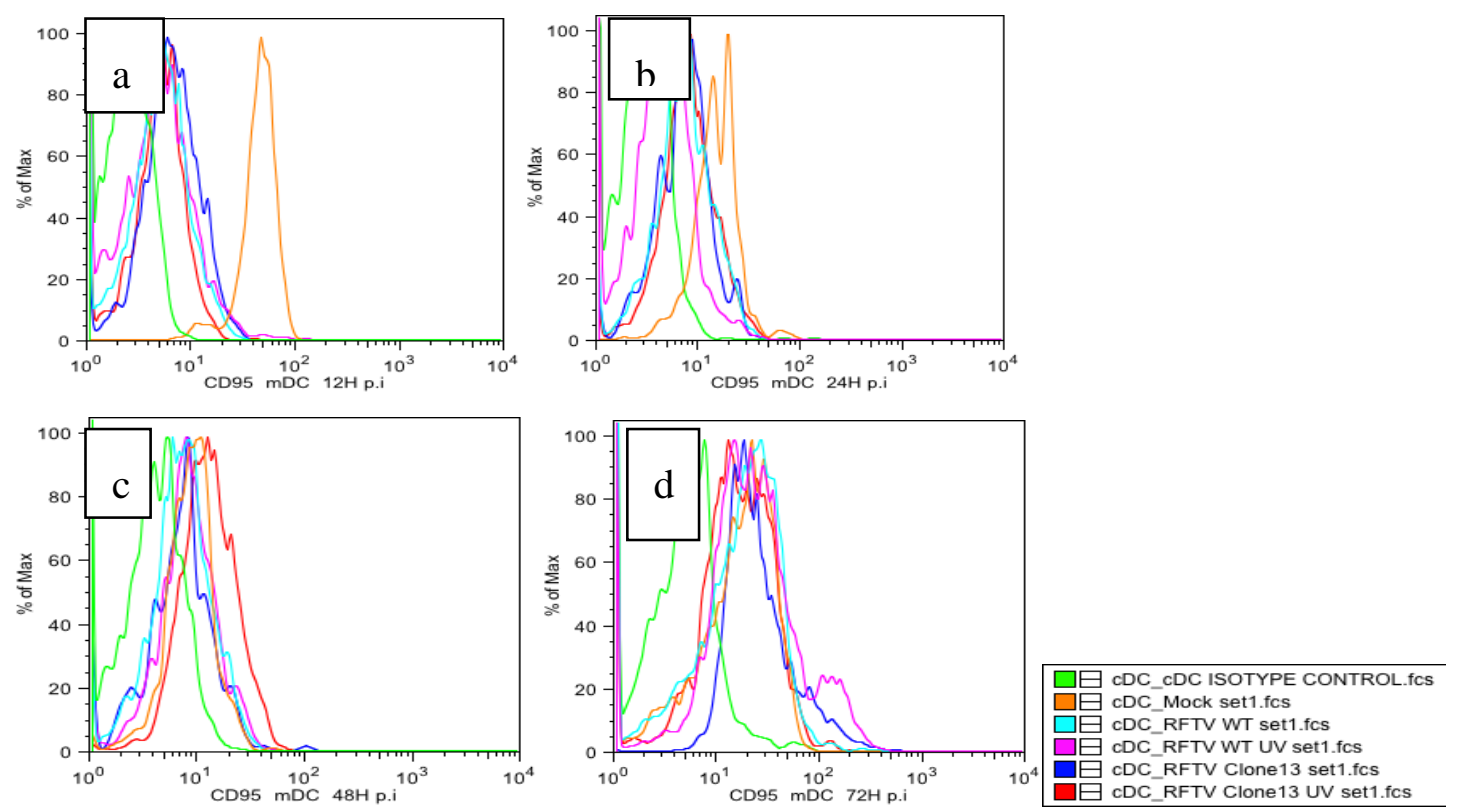

Figure 42. CD95 regulation after an infection of mDCs by RVFV.
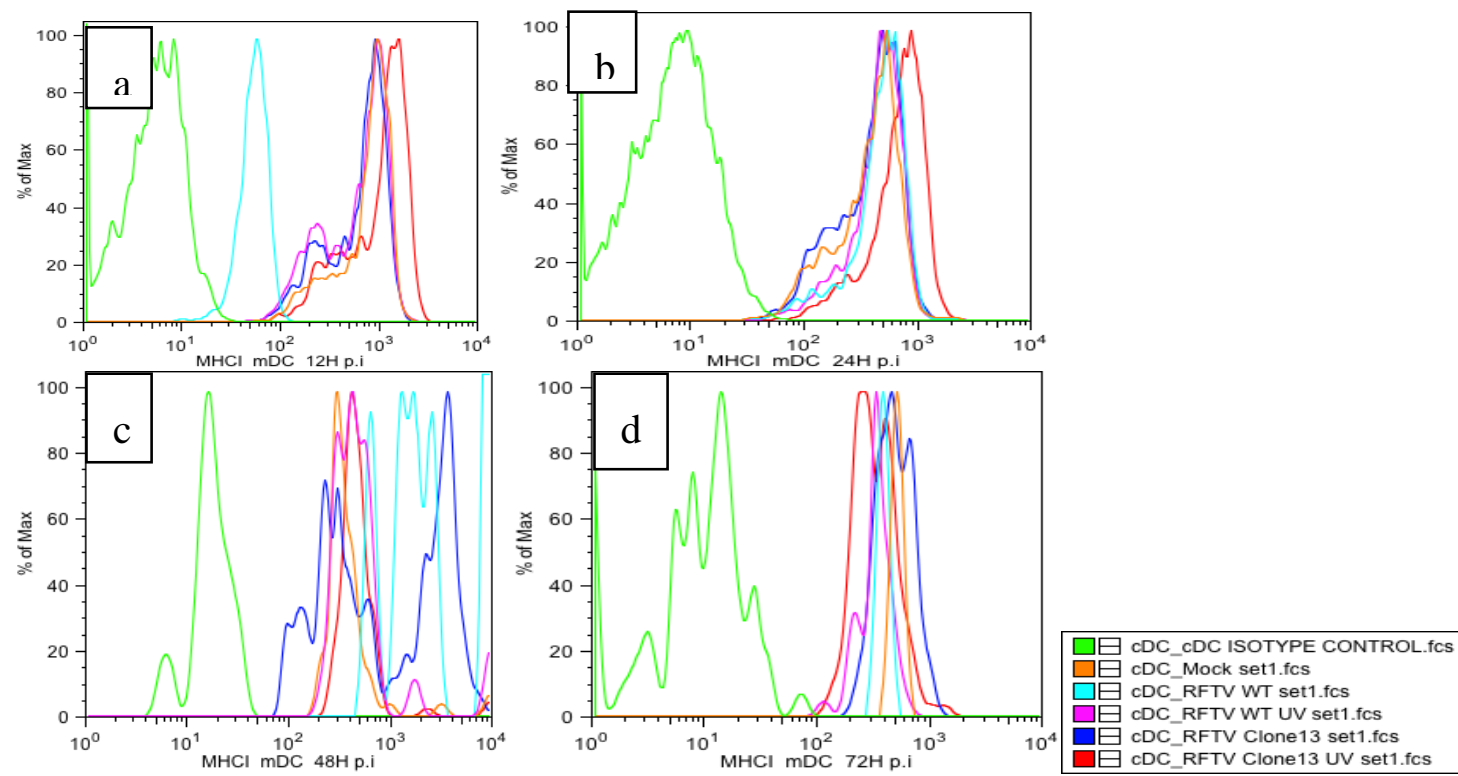

Figure 43. MHCI regulation after an infection of mDCs by RVFV. 

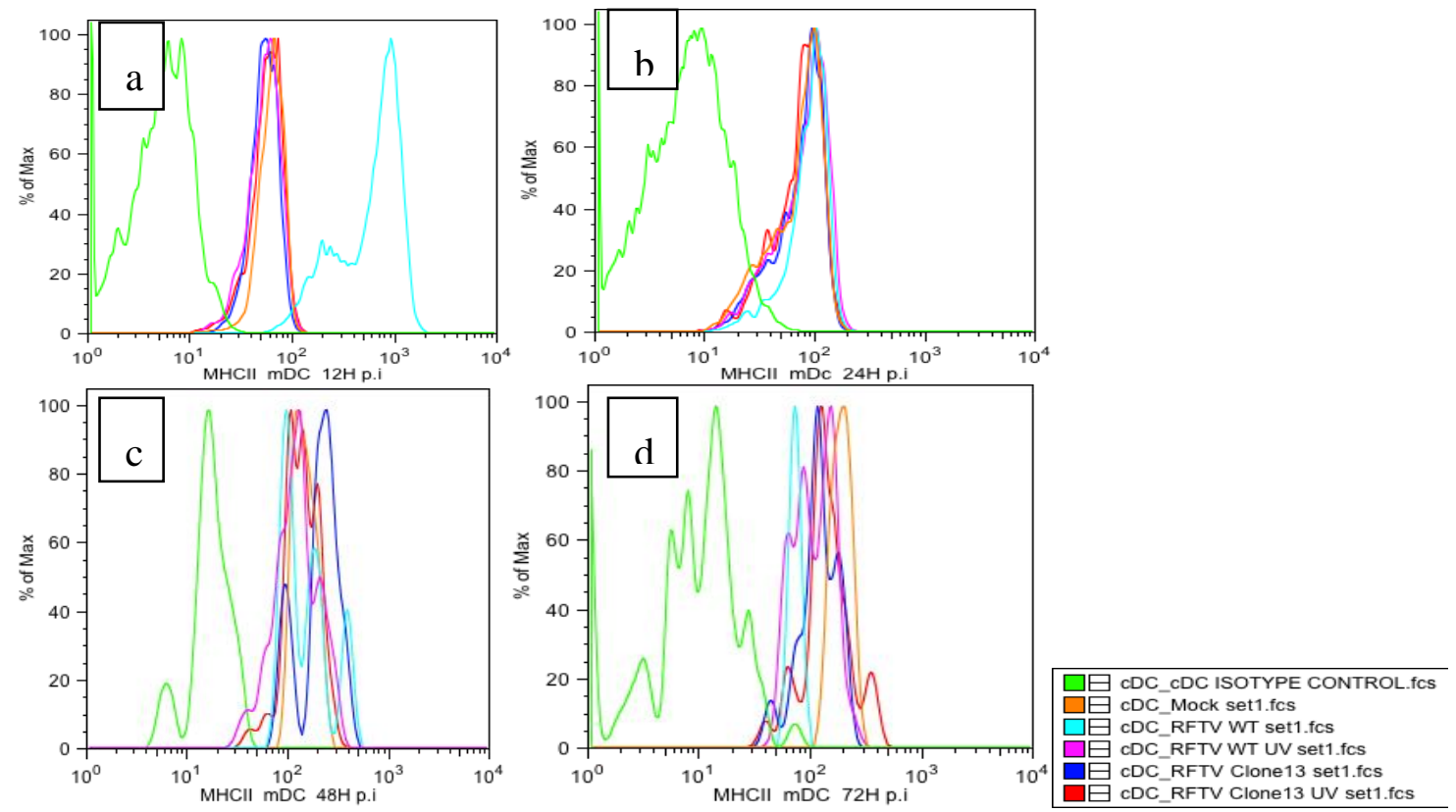

Figure 44. MHCII regulation after an infection of $\mathrm{mDCs}$ by RVFV.

\subsection{Additionnal results of expression and regulation of surface marker molecules in pDCs infected by RVFV (chapter 3.11)}

In all figures results are indicated as follows: infection with wtRVFV (purple), UV inactivated wtRVFV (light blue), RVFV clone 13 (dark blue), UV inactivated RVFV clone 13 (red), and mock control (orange) at 12h, 24h, 48h, and 72h (clockwise).
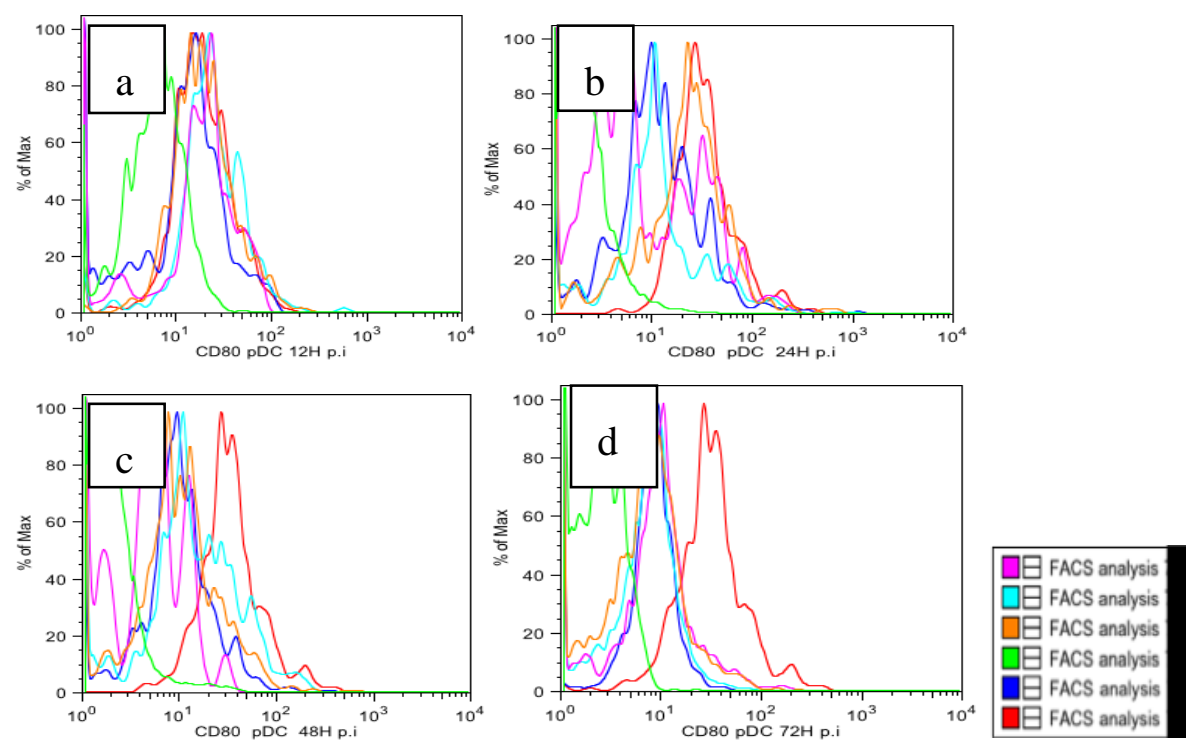

RFTV UV set2 fos RFTV set1.fes Mock set1.fes Isotyp.tes Clone13 set1.fos Clone 13 UV set1.fcs

Figure 45. CD80 regulation after an infection of pDCs by RVFV. 

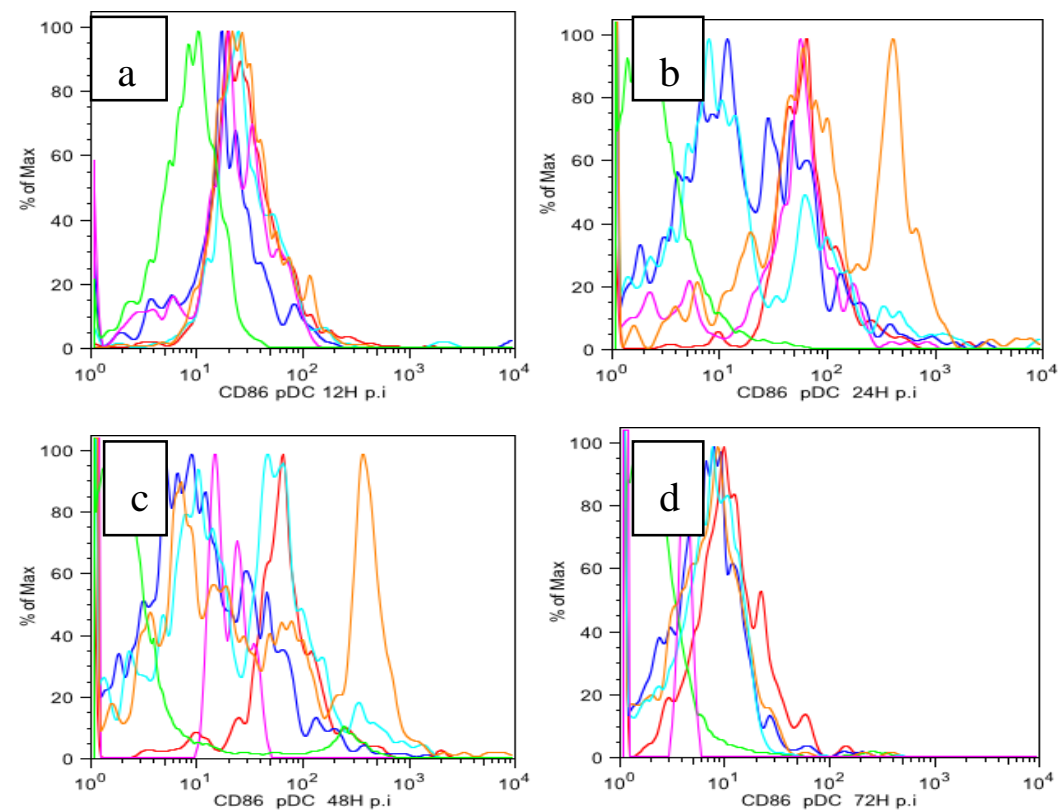

$\square$ 口 FACS analysis
$\square$ FACS analysis
$\square$ FACS analysis
$\square$ FACS analysis
$\square$ FACS analysis
$\square$ FACS analysis

RFTV UV set2 fes

RFTV set1.fes

Mock set1.fes

Isotyp.fes

Clone13 set1.fes

Clone 13 UV set1.fos

Figure 46. CD86 regulation after an infection of pDCs by RVFV.
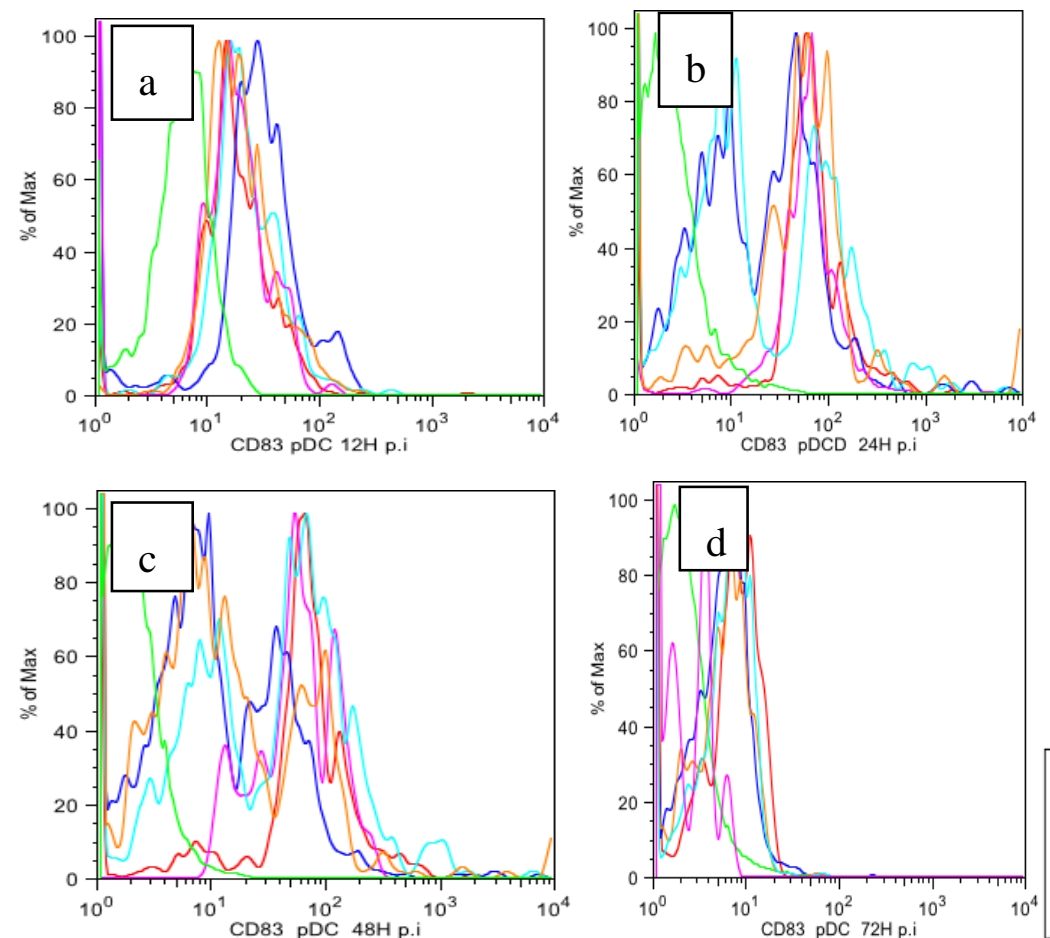

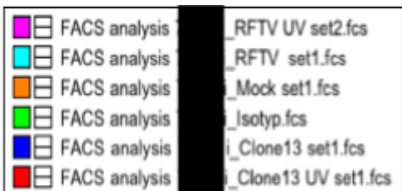

Figure 47. CD83 regulation after an infection of pDCs by RVFV. 

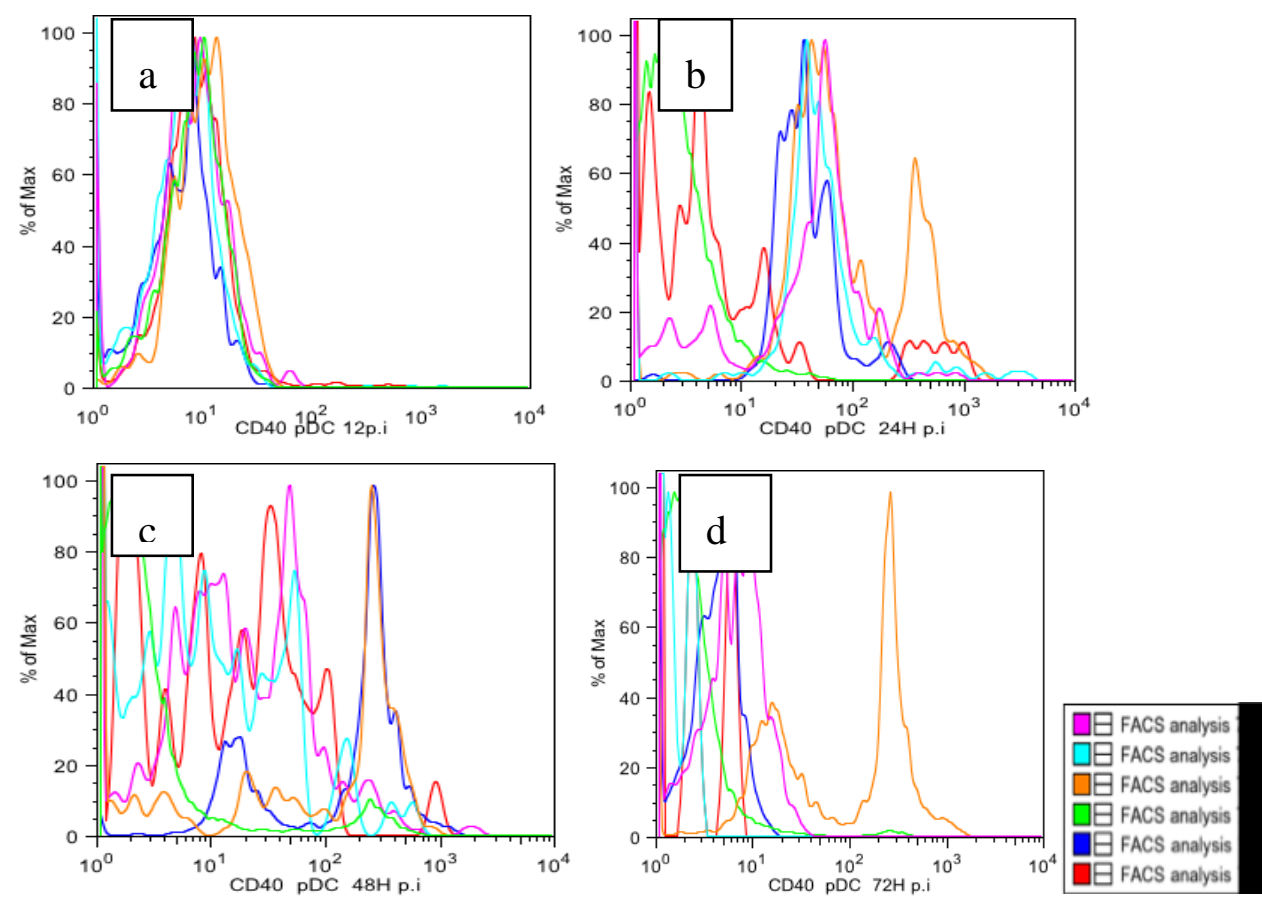

RFTV UV set2 fes RFTV set1.fes Mock set1.fes Isotyp.tcs

Figure 48. CD40 regulation after an infection of pDCs by RVFV.
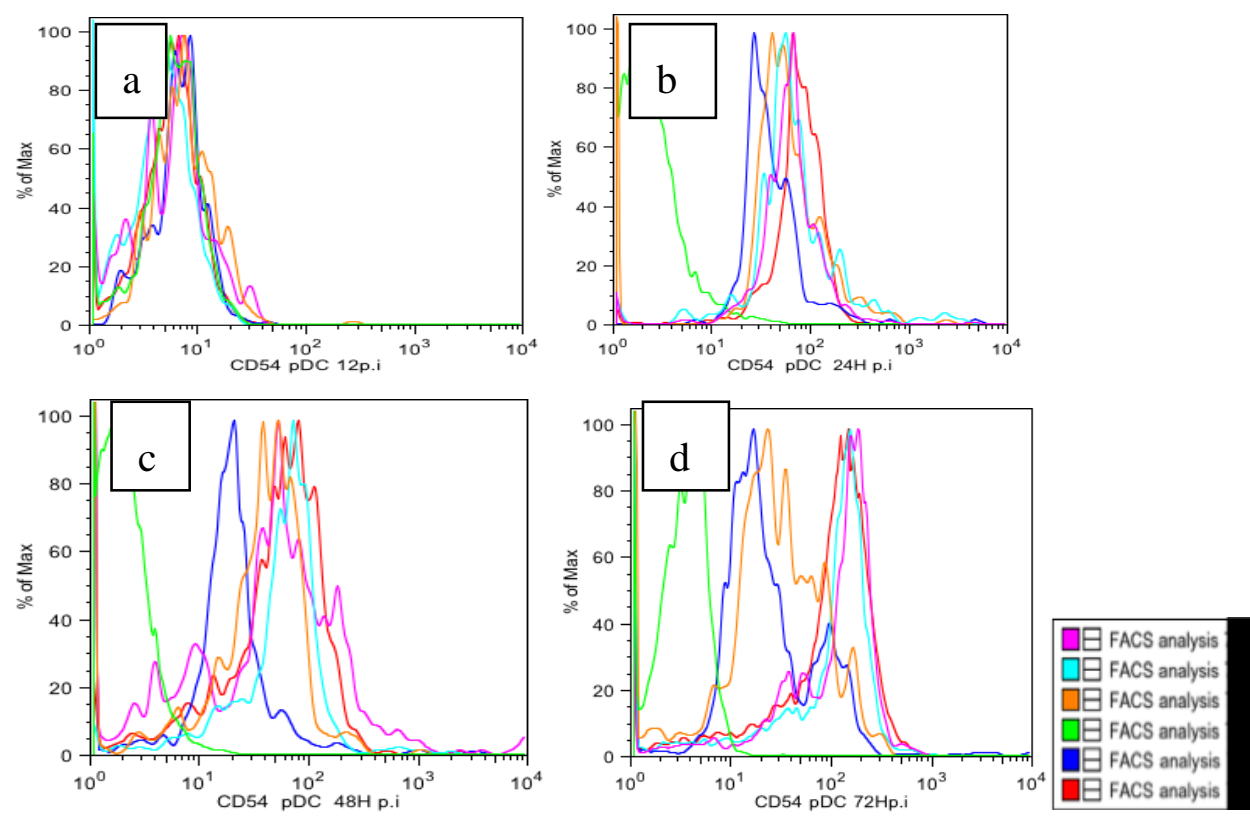

RFTV UV set2.fes RFTV set1.fes Mock set1.fes

Isotyp.fes

Clone 13 set1.fos

Clone 13 UV set1.fes

Figure 49. CD54 regulation after an infection of pDCs by RVFV. 

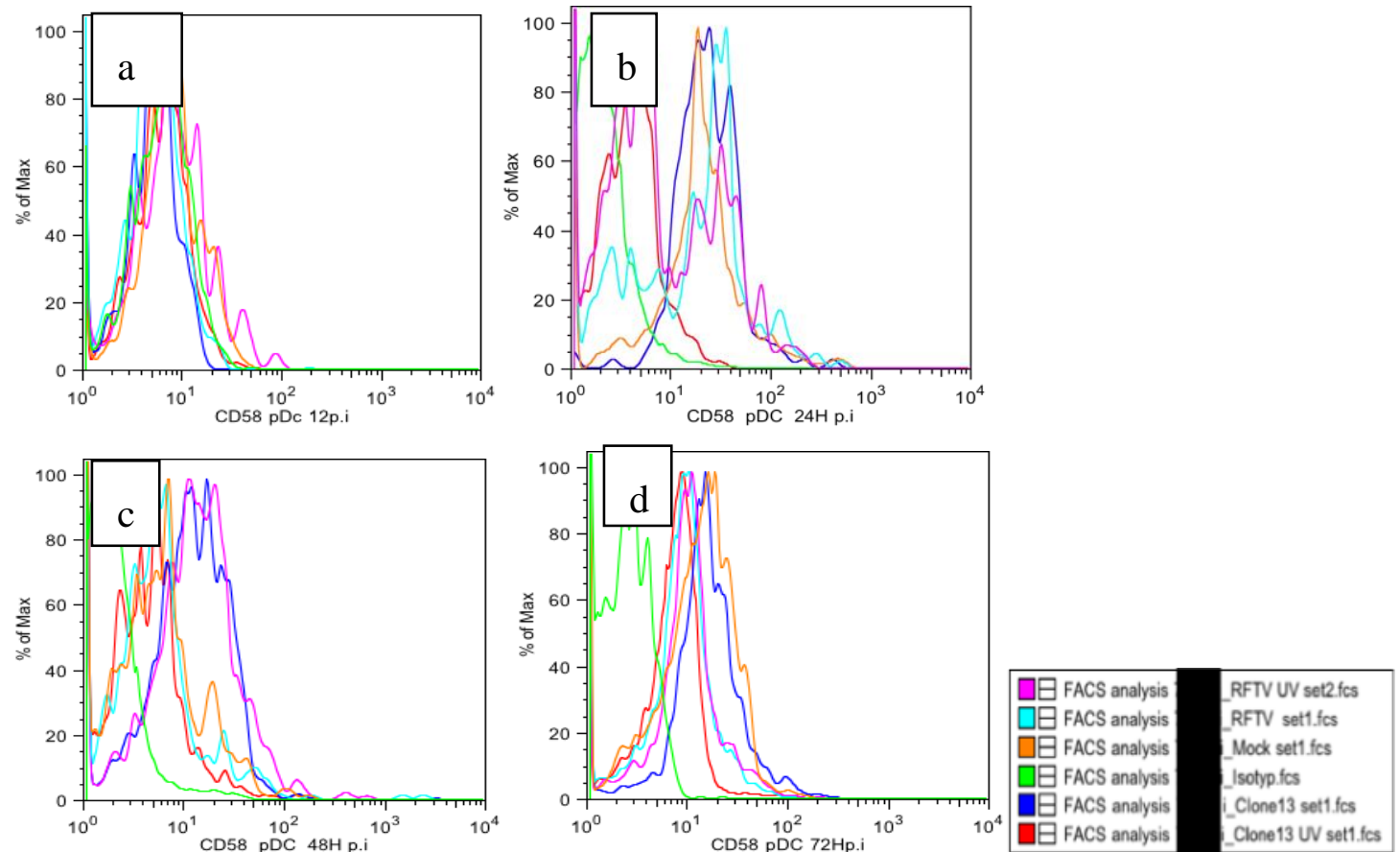

Figure 50. CD58 regulation after an infection of pDCs by RVFV.
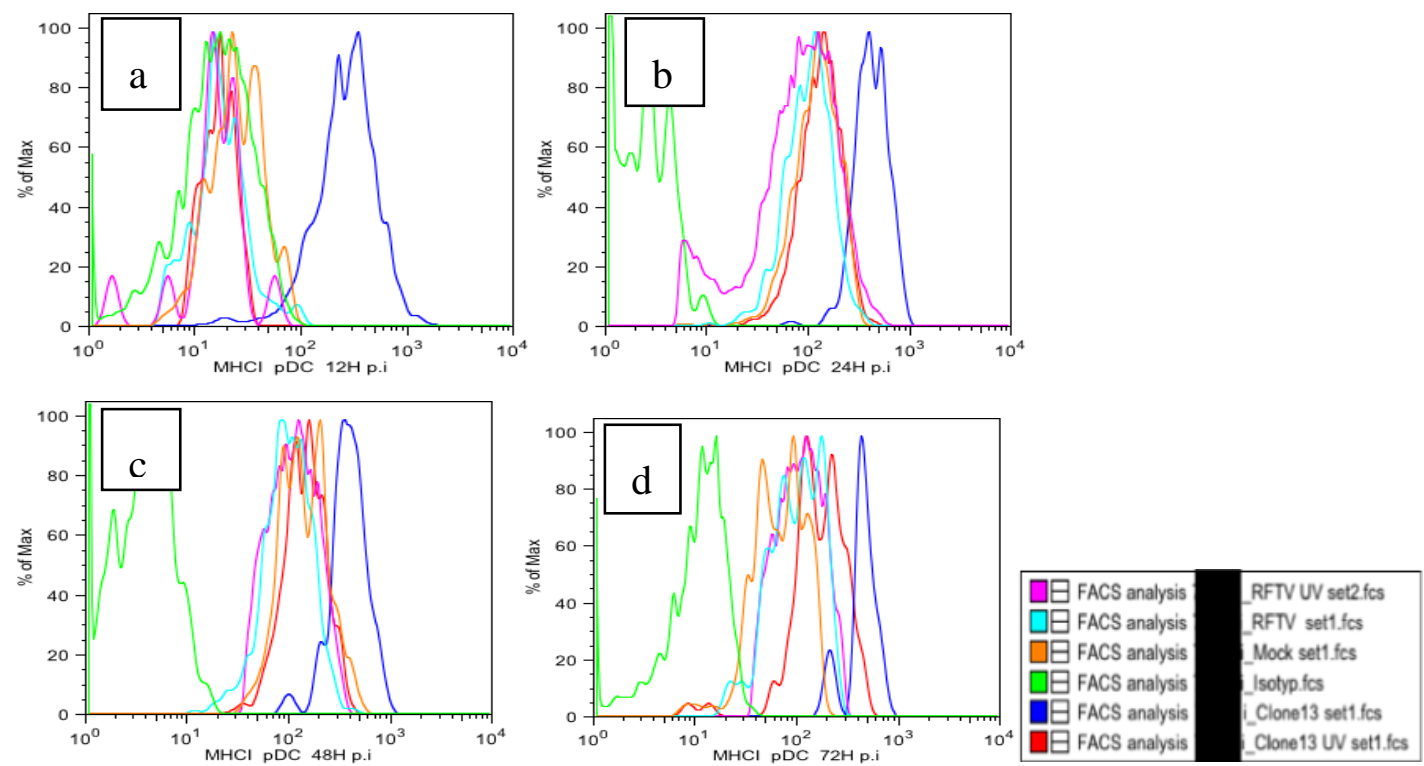

Figure 51. MHCI regulation after an infection of pDCs by RVFV. 

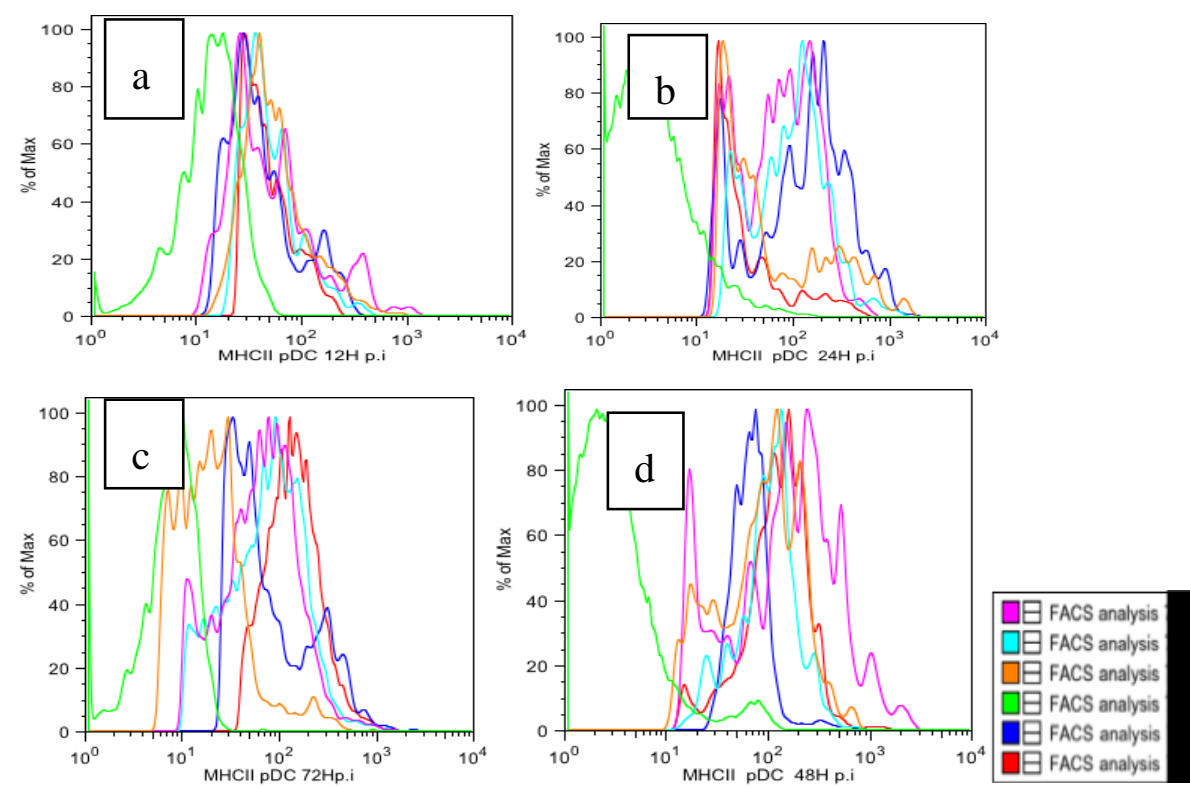

RFTV UV set2 fes RFTV set1.fes Mock set1.fes Isotyp.tes Clone13 set1.fes Clone 13 UV set1.fos

Figure 52. MHCII regulation after an infection of pDCs by RVFV.
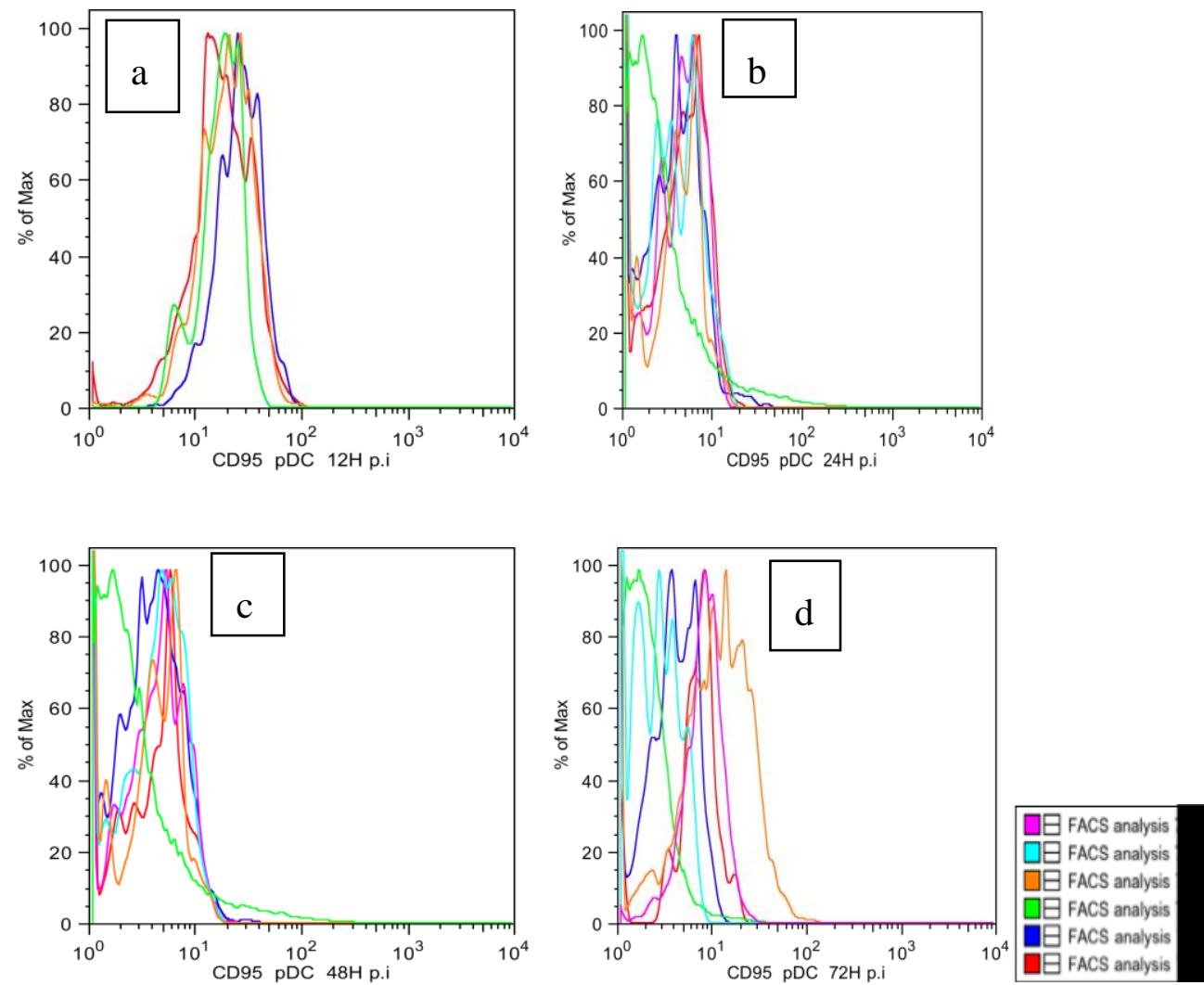

RFTV UV set2. fes RFTV set1.fes Mook set1.fes Isotyp. fes Clone13 set1.fos Clone 13 UV set1.tes

Figure 53. CD95 regulation after an infection of pDCs by RVFV. 


\subsection{Complete alignment of NSs sequences of the 26 RVFV strains analyzed in} this thesis (Chapters 3.4 and 3.5)
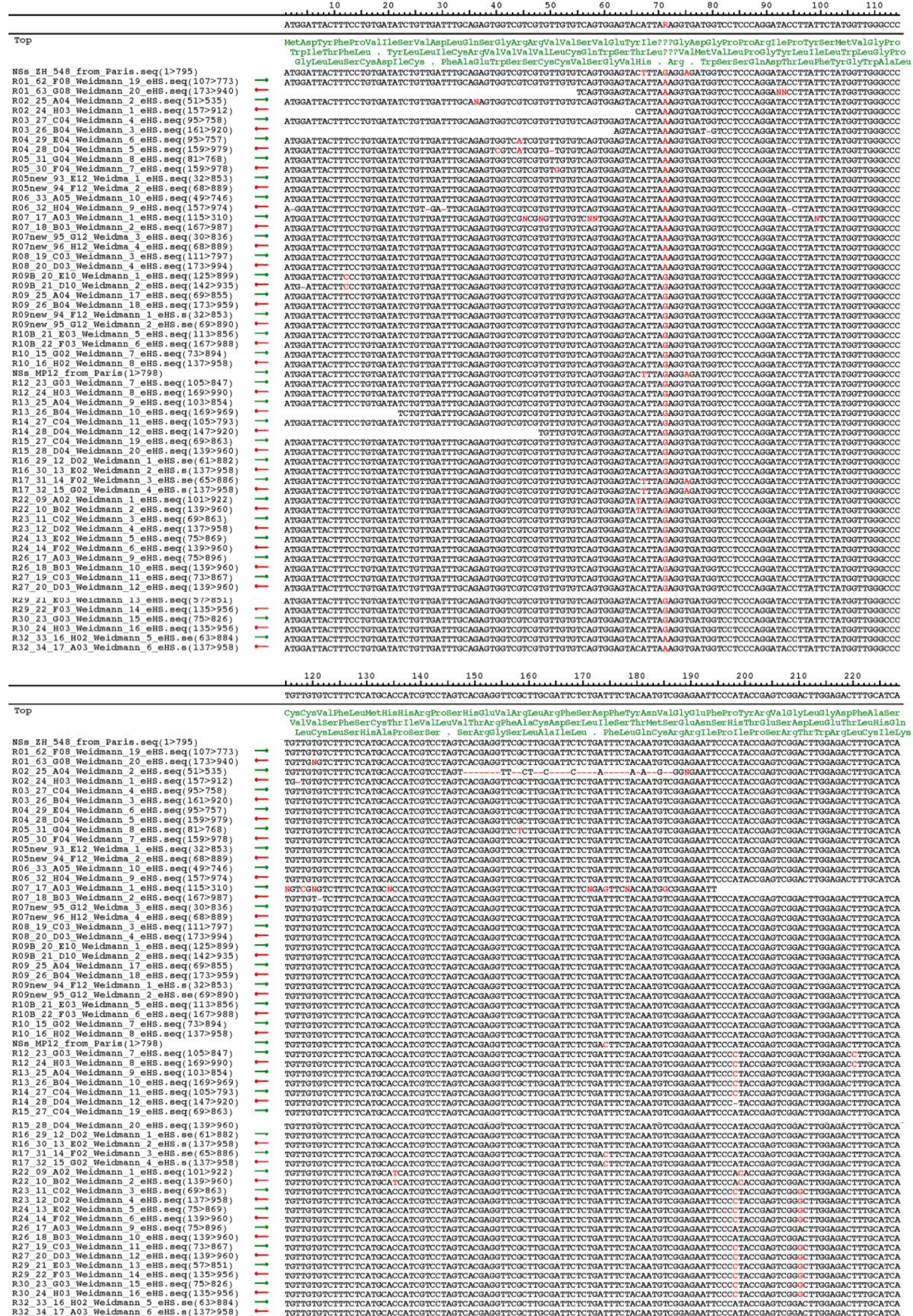

تسلس

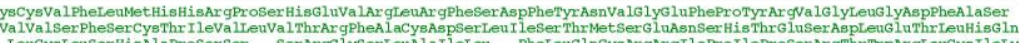

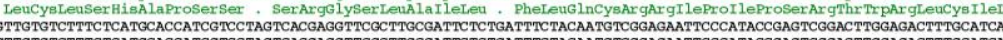

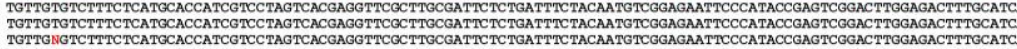

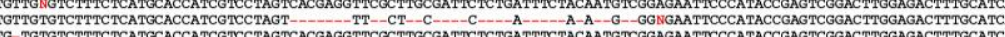

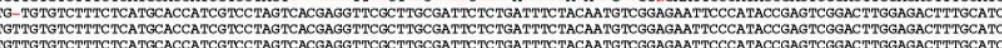
GTTGTGTCCTTCTCATGCACCATCGTCCTAGTCACGAGG TTCGC TTGCGATTCTCTGATTTC TACAATGTCGGAGAATTCCCATACCGAGTCGGACTTGGAGACTTTGCATC

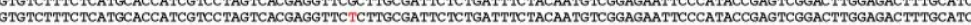

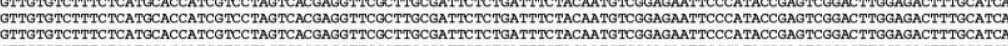

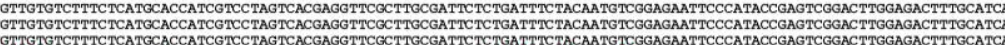

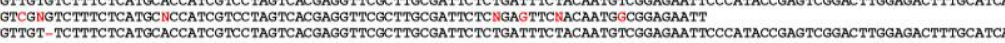

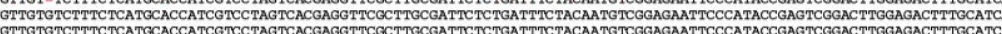
GTTGTGTCTYTCTCATGCACCATCGTCC TAGTCACGAGGTTCGC TTGCGATTCTCTGATTTCTACAATGTCGGAGAATTCCCATACCGAGTCGGACTTGGAAACTTTGCATCA

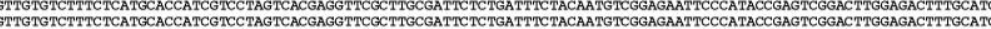

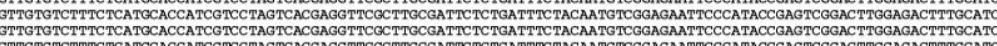

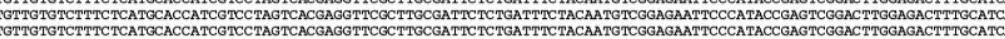
TTGTGTCTTTC TCATGCACCATCGTCC TAGTCACGAGGTTTCGCTTGCGATTC TCTGATTTC TACAATGTCGGAGAATTCCCATACCGAGTCGGACTTGGAGACTTTGCATC

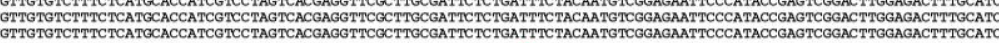

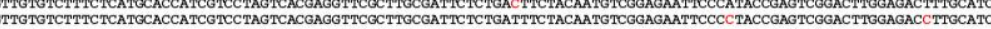
GTTGTGTCTTTTCTCATCCACCATCGTCCTAGTCACGAGGTTCGCTTGCGATTCTCTGATTTCTACAATTGTCGGAGAATTCCCCTACGAAGTGGGACTTGGAGACACTTTGGATCA GTTGTGTCTTTCTCATGCACCATCGTCCTAGTCACGAGGTTCGCTTGCGATTCTCTGATTTCTACAATGTCGGAGAATTCCCCTACCGAGTCGGACTTGGAGACTTTGCATCA

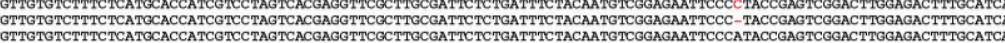

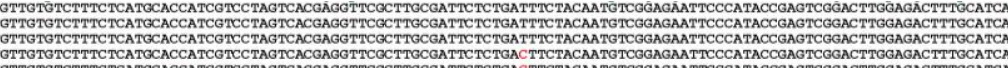
GTTGTGTCTYTCTCATECAC

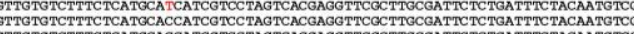
GTTGTTCTCTYTCTAATGCAC

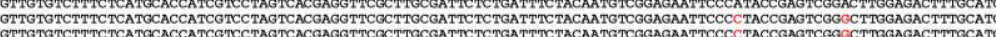

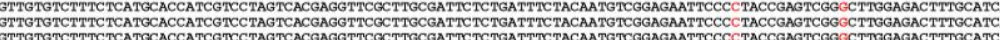

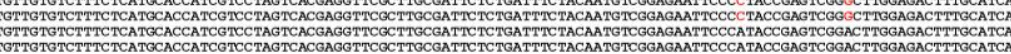


مُ20 230 AACGTTOCACCTCCACCAGCAAAGCCTTTTCAGAGACTTATTGATCTAATAGGCCATATGACTCTYAGTGATTTCACAAGGTTCCCCAATCTRAAAGAAGCCATATCCTGGCCT

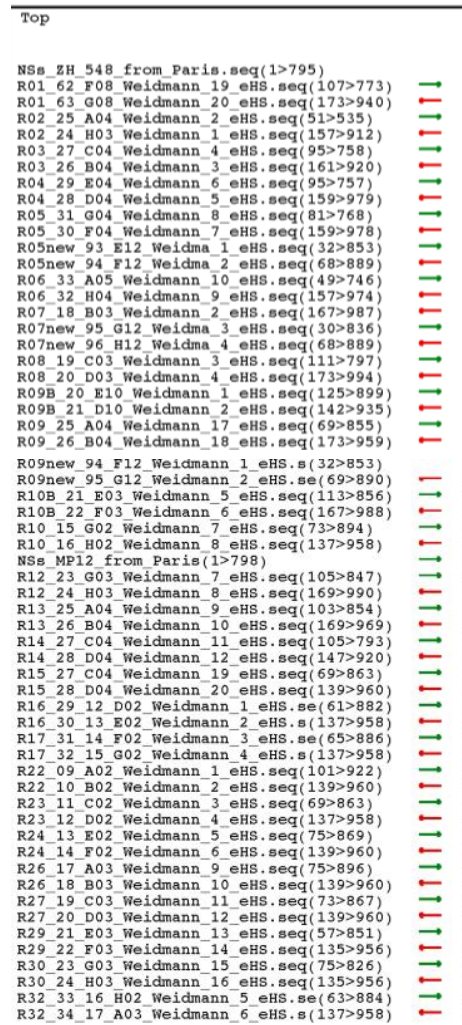

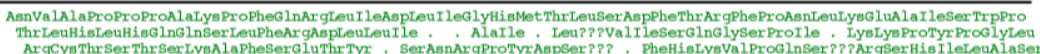

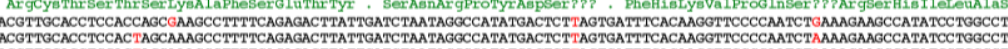
ACGTTGCACCTCCACTACCANGCCCTTTTCAGAGACTTATTEATCCTAATAGGCCATATGACTCTTAGTGATTTCACAAGGTTCCCCAATCTAAAAAGAAGCCATATCCCTGGCC

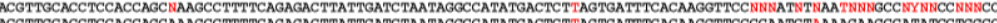
AGTGATTTCACAAGGTTCCCCA

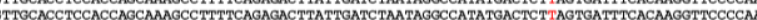

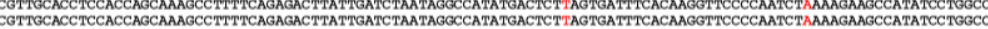
MCGTTGCACCTCCACCAGCAAAGCCTTTTCAGAGACTTATT GATCTAATAGGCCATATGACTCTTAGTGATTTCACAAGGTTCCCCAATCTAAAAGAAGCCATATCCTGGCC ACGTTOCACCTCCACCAGCAAGCCCTTTTCAGAGACTTATTGATCCAATAGGCCATATGACTCTTAGTGATTTCACAAGGTCCCCAATCTAAAAGAAGCCATATCCTGGCC ACGTTTCCACCTCCACCAGCAAAGCCTTTTCACAGAGACTTATTGATCTAATAGGCCATATGACTCTTAGTGATTTCACAAGGTTCCCCAATCTAAAAGAAGCCATATCCTGGCC

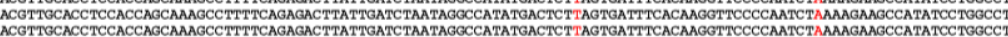

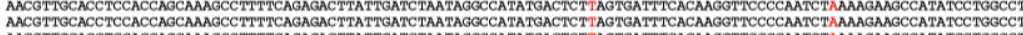
AACGTTGCACCTCCACCAGCAAAGCCTTTTCAGAGACTTATTGATCTAATAGGCCATATGACTCTTAGTGATTTCACAAGGTTCCCCAATCTAAAAGAAGCCATATCCTGGCC AACGTTGCACCTCCACCAGCAAAGCCTTTTCAGAGACTTATTGATCTAATAGGCCATATGACTCTCAGTGATTTCACAAGGTTCCCCAATC TGAAAGAAGCCGTATCCTGGCCT
AACGTTGCACCTCCACCAGCAAAGCCTTTTCAGAAACTTATTGATCTAATAGGCCATATGACTCTCAGTGATTTCACAAGGTTCCCCAATCTGAAAGAAGCCGTATCCTGGCCT ACGTTGCACCTCCACCAGCAAAGCCTTTTCAGAGACTTATTGATCTAATAGGCCATATGACTCTCAGTGATTTCACAAGGTTCCCCAATCTGAAAGAAGCCGTATCCTGGCC AACGTTGCACCTCCACCAGCAAAGCCTTTTCAGAGACTTATTGATCTAATAGGCCATATGACTCTCAGTGATTTCACAAGGTTCCCCAATCTGAAAGAAGCCGTATCCTGGCCT AACGTTCACCCCCCACCAGCAAAGCCTTTTCAGAGACTTAATGAACTAATAGCCCATATGACTCTCAGTGATTTCACAAGGTTCCCCAATCTGAAAGAAGCCGTATCCTGGCC ACGTTGCACCTCCACCAGCAAAGCCTTTTCAGAGACTTATTGATCTAATAGGCCATATGACTCTCAGTGATTTCACAAGGTTCCCCAATCCGAAAGAAGCCGTATCCTGGCC

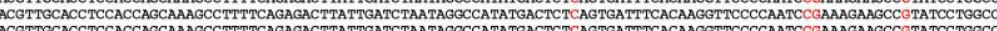
AACGTTGCACCTCCACCAGCGAAGCC TTTTCAGAGACTTATTGATCTAATAGGCCATATGACTCTTAGTGATTTCACAAGGTTCCCCAATCTGAAAGAAGCCATATCCTGGCC

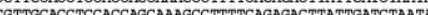

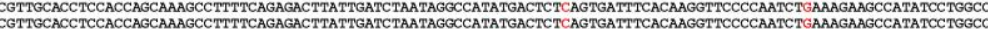

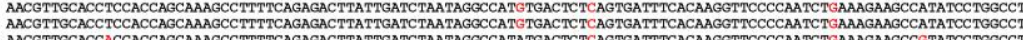
AACGTTOCACCACCACCAGCAAAGCC TTTTCAGAGACTTATTGATCTAATAGGCCATATGACTCTCAGTEATTTCACAAGGTTCCCCAATCTEAAAGAAGCCGTATCCTGGCC AACGTTGCACCACCACCAGCAAAGCC TTT TCAGAGACT TATTGATC TAATAGGCCATATGACTC TCAGTGATTTCACAAGGTTCCCCAATC TGAAAGAAGCCGTATCCTGGCCI
AACGTTGCACCACCACCAGCAAAGCCTTTTCAGAGACTTATTGATCTAATAGCCCATATGACTCTCAGTGATTTCACAAGGTTCCCCAATCTGAAAGAGCCGTATCCTGGCCT AACGTTSCACCACCACCAGCAAAGCCTTTTCAGAGACTTATTGATCTAATAGGCCATATGACTCTCAGTGATTTCACAAGGTTCCCCAATCTGAAAGAAGCCGTATCCTGGCC AACGTTGCACCTCCACCAGCGAAGCCTTTTCAGAGACTTATTGATCTAATAGGCCATATGACTCTTAGTGATTTCACAAGGTTCCCCAATCTGAAAGAAGCCATATCCTGGCC
AACGTTGCACCTCCACCAGCGAAGCCTITTCAGAGACTTATTGATCTAATAGGCCATATGACTCTTAGTGATTTCACAAGGTTCCCCAACTGAAAGAAGCCATATCCTGGCC

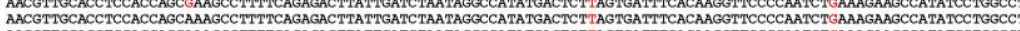
AACGTTECACCTCCACCAGCAAAGCCTTTTCAGAGACTTATTGATCTAATAGGCCATATGACTCTTAGTGATTTCACAAGGTTCCCCAATCTGAAAGAAGCCATATCCTGGCC AATGTTGCACCTCCACCAGCAAAGCCTTTTCAGAGACTTATTGATCTAATAGGTCATATGACTCTTAGTGATTTCACAAGGTTCCCCAATCTGAAAGAAGCCATATCCTGGCC AATGTTGCACCTCCACCAGCAAAGCCTTTTCAGAGACTTATTGATCTAATAGGTCATATGACTCTTAGTGATTTCACAAGGTTCCCCAATCTGAAAGAAGCCATATCCTGGCC AATGTTGCACCTCCACCAGCAAAGCCTTTTCAGAGACTTATTGATCTAATAGGTCATATGACTCTTAGTGATTTCACAAGGTTCCCCAATCTGAAAGAAGCCATATCCTGGCC
AACGTTGCACCTCCACCAGCAAAGCCTTTTCAGAGACTTATTGATCTAATAGGCCATATGACTCTTAGTGATTTCACAAGGTTCCCCAATCTGAAAGAGGCCATATCCTGGCC AACGTTCCACCTCCACCAGCAAAGCCTTTTCAGAGACTTATTGATCTAATAGGCCATATGACTCTTAGTGATTTCACAAGGTTCCCCAATCTGAAAGAAGCCATATCCTGGCC AATGTTECACCTCCACCAGCAAAGCCTTTTCAGAGACTTATTGATCTAATAGGTCATATGACTCTTAGTGATTTCACAAGGTTCCCCAATCT GAAAGAAGCCATATCCTGGCC AATGTTGCACCTCCACCAGCAAAGCCCTTTTCAGAGACTTATTGATCTAATAGGTCATATATACTCTTAGTGATTTCACAAGGTTCCCCAATCTGAAAGAAGCCATATCCTGGCC AATGTTECACCTCCACCAGCAAAGCCTTTTCAGAGACTTATTGATCTAATAGGTCATATGACTCTTAGTGATTTCACAAGGTTCCCCAATCTGAAAGAAGCCATATCCTGGCC

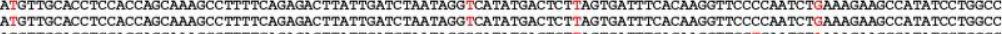
AACGTTCCACCTCCACCAGCAAAGCCTTTTCAGAGACTTATTGATCTAATAGGCCATATGACTCTTAGTGATTTCACAAGGTTCCTCAATCTAAAAGAAGCCATATCCTGGCC
AACGTTGCACCTCCACCAGCAAAGCCTTTTCAGAGACTTATTGATCTAATAGGCCATATGACTCTTAGTGATTCACAAGGTTCCTCATCTAAAAGAAGCCATATCCTGGCC

\begin{tabular}{|c|c|c|}
\hline & & 440 \\
\hline & & TEA \\
\hline 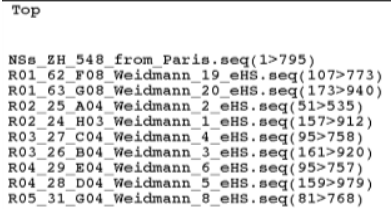 & $\begin{array}{l}\Xi \\
\Xi \\
\Xi \\
\Xi\end{array}$ & 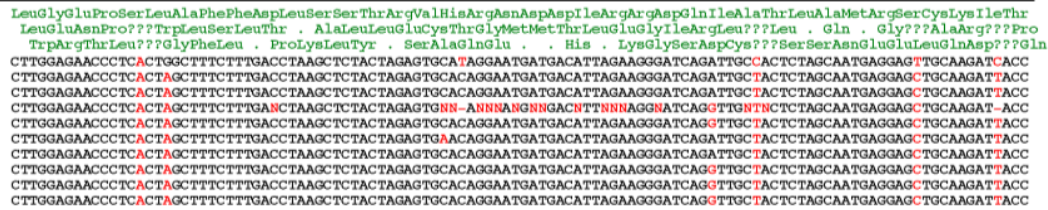 \\
\hline R05_30_F 04 Weidmann_7_eHS. seq $(159>978)$ & & 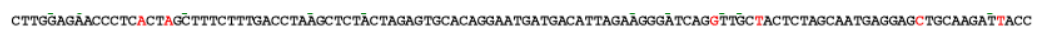 \\
\hline & & \\
\hline 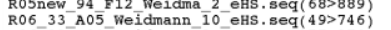 & & \\
\hline 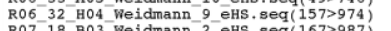 & & \\
\hline 3 Weic & & \\
\hline & & CTAC $\triangle 2>C$ \\
\hline idmann_- eHs. & & ACC \\
\hline & & $\begin{array}{l}\mathrm{AcC} \\
\mathrm{ACC}\end{array}$ \\
\hline $\begin{array}{l}\text { lidmann } 1 \text { - } \\
\text { eidmann } 2\end{array}$ & & CTAC $\mathrm{C}, \mathrm{C}$ \\
\hline 5_A 04 - Weidmann 17 - eH & & CCTI \\
\hline 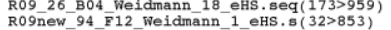 & & \\
\hline 95 - 612 - Fe idmann -2 eHS. se & & CAATGA \\
\hline R10 & & \\
\hline idmann -7 - ${ }^{2}-\bar{e}$ & & TAGCAATGAGC \\
\hline 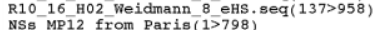 & & AcC \\
\hline & & \\
\hline & & HEGTECACAGGAATGAIGALATIA \\
\hline eHS.s & & \\
\hline R13 26 B 04 -Weidma & & \\
\hline R14 & & GCA \\
\hline 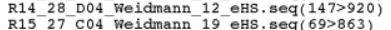 & & \\
\hline 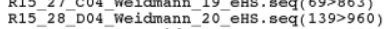 & & \\
\hline D02 W & & \\
\hline $116-30-13$-E02-Weidr & & cc \\
\hline $\mathrm{R} 11_{3}-31-14 \mathrm{FO}_{2}$-Weic & & CA \\
\hline 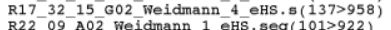 & & \\
\hline mann & & \\
\hline $\ln ^{-2}-e^{2}$ & & CC \\
\hline 2_D D2_Weid & & cc \\
\hline R24_13_E02 Weid & & GAGTTGCA \\
\hline 02 2 & & GAGTTGCA \\
\hline $\mathrm{BO}_{3}-\mathrm{I}$ & & \\
\hline $\begin{array}{l}\text { BO3 Weid } \\
\mathrm{CO}^{-} \text {Weid. }\end{array}$ & & \\
\hline DO3-Weidmann-12 eHS. seqd & & \\
\hline E03-Weidmann_13 eHs. seq ( 57 & & TRGCCACT \\
\hline Fo3 Weidmann 14 eH & & \\
\hline Weidman & & \\
\hline & & ССТАСТСТВ - \\
\hline & & \\
\hline
\end{tabular}




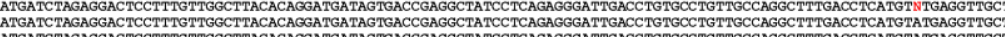

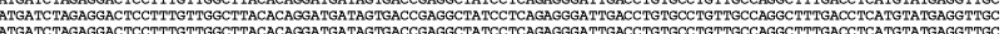

ATGATC TAGAGGACTCCTTTGTTGGCTTACACAGGATGATAGTGACCGAGGCTATCCTCACAGGGATTGACCTGTGCCTGTTGCCAGGCTTTGACCTCATGTATGAGGTTGCT

AATGATCTAGAGGACTCCTTTGTTGGCTTACACAGGATGATAGTEACCGAGGCTATCCTCAGAGGGATTGACC TGTGCCTGTTGCCAGGC TTTEACC TCA TGTATGAGGTTGC

ATGATCTAGAGGACTCCTTTGTTGGCTTACACAGGATGATAGTGACCGAGGCTATCCTCAGAGGGATTGACCTGTGCCTGTTGCCAGGCTTTGACCTCATGTATGAGGTTGC

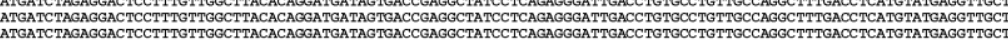

AATGATC TGGAAGATTCCTTTGTTGGCTTACACAGGATGATAGTGACCGAGGCTATTCTCAGAGGAATTGACCTGTGCCTGTTGCCAGGCTTTGANCTCATGTATGAGGTTGC

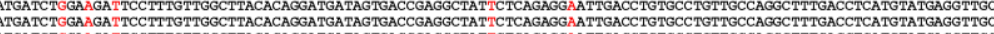

ATGATCTGGAAGATTCCTTTGTTGGCTTACACAGGATGATAGTGACCGAGGCTATTCTCAGAGGATTGACCTGTGCCTGTTGCCAGGCTTTGACCTCATGTATGAGGTTGC

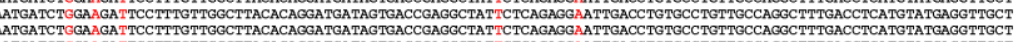

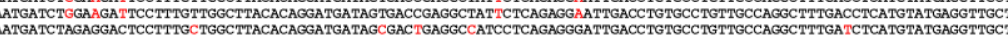

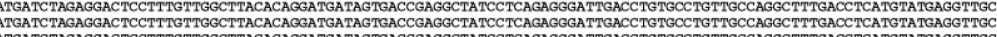

ATGATCTAGAGGACTCCTTTGTTGGCTTACACAGGATGATAGTEACCGAGGCTATCCTCAGAGGGATTGACCTGTGCCTGTTECCAGGCTTTGACCTCATGTATTAGGTTGC

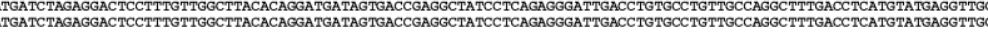

AMGATCTGGAAGATTCCTTTGTTGGCTTACACAGGATGATAGTGACCGAGGCTATTCTCAGAGGATTTGACCTGTGCCTGTTGCCAGGCTTTGACCTCATGTATGAGGTTGC

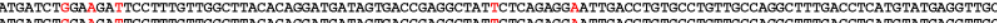

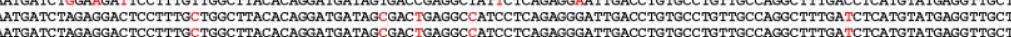

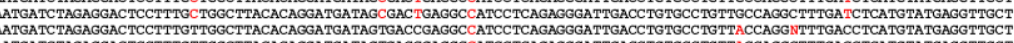

ATGATCTAGAGGACTCCTTTGTTGGCTTACACAGGATGATAGTGACCGAGGCATCCTCAGAGGGATTGACCTGTGCCTGTTAACAGGCTTTGACCTCATGTATGAGGTTGC

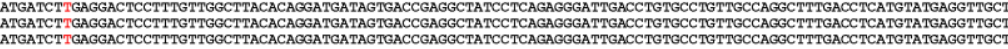

ATGATCTT GAGGACTCCTTTGTTGGCTTACACAGGATGATAGTGACCGAGGCTATCCTCAGAGGGATTGACC TGTGCCTGTTGCCAGGCTTTGACCTCATGTATGAGGTTGC

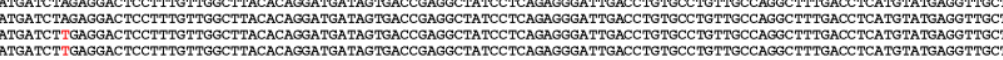

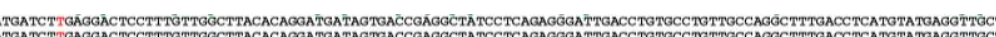

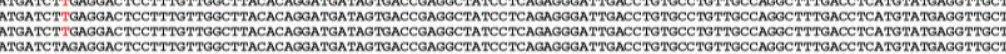

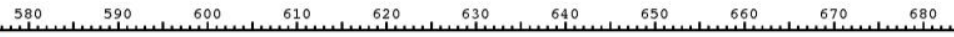

\begin{tabular}{|c|c|c|}
\hline Top & & 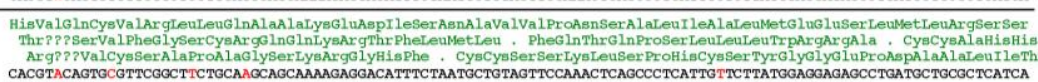 \\
\hline & & \\
\hline 1-63-608-Weidmann-20 eHS & & 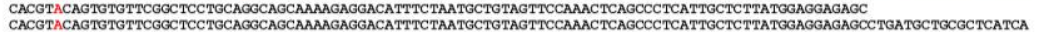 \\
\hline H03 Weidmann_ 1 - EHS. seq (157>912) & & TTCCT \\
\hline & & (CCG \\
\hline 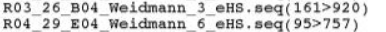 & & \\
\hline 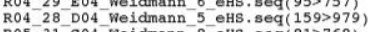 & $\vec{a}$ & $\begin{array}{l}\text { ETCATTGGTCTAATGG } \\
\text { CTCATTCCTCTATGGAGAGAGCC: }\end{array}$ \\
\hline 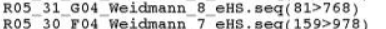 & & \\
\hline 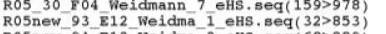 & $\leftrightarrows$ & 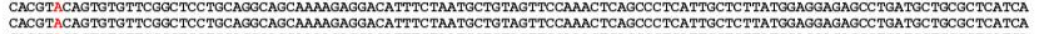 \\
\hline 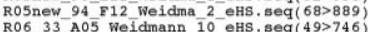 & & 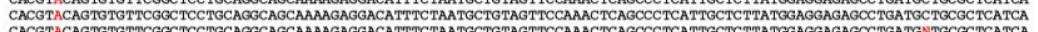 \\
\hline 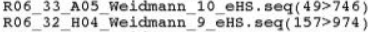 & & \\
\hline 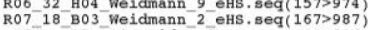 & & \\
\hline w-95 612 - Weidma 3 e eHs & & $\begin{array}{l}\text { TAATCTGTGATTCCAAACTAGCC } \\
\text { TAATGCTGTAGTTCAAACTCAGC }\end{array}$ \\
\hline lew_96- 12 Weidma 4 eHs. seq & & CGGCTCCTGCAGGCAGCAAAAGAGGACA? \\
\hline $\begin{array}{l}19-\mathrm{CO}^{3} \text { Weidmann } \\
20\end{array}$ & & \\
\hline $\begin{array}{l}30 \text { Weidmann } 44 \text { eH } \\
\text { Weidmann } \\
\end{array}$ & & \\
\hline 35) & & TCA \\
\hline 25-A04-Weidmann 17 - eH & & A A \\
\hline $\begin{array}{l}\text { idmann_18 } \\
\text { Weidmann }\end{array}$ & & \\
\hline 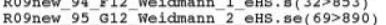 & & $=A$ \\
\hline $10 \mathrm{~B} 21 \mathrm{EO} 3$ Weidmann 5 e EAs. & & TCA \\
\hline $\mathrm{R} 10 \mathrm{O}-22 \mathrm{FO}$ - Weidmann 6 eHS. & & TCA \\
\hline 210_15_G02_Weidmann_7_eH & & \\
\hline MP: & & $\begin{array}{l}\text { OCTCCTGCCGGGCACA } \\
\text { SCTTCTGCAAGCAGCA }\end{array}$ \\
\hline 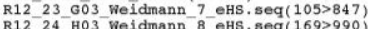 & & \\
\hline 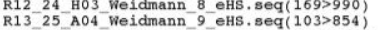 & & \\
\hline 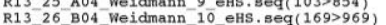 & & TCA \\
\hline 27 C04 Weidmann 11 e & & TCA \\
\hline R14_28_D 04 Weidmann_12_eH & & \\
\hline & & ЕТGСGCTCATCA \\
\hline R15-28-D04 Weidr & & \\
\hline 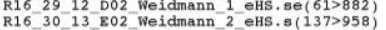 & & 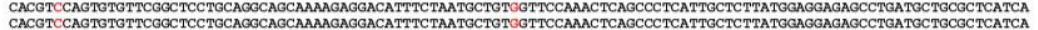 \\
\hline 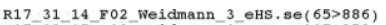 & & \\
\hline & & \\
\hline & & \\
\hline & & $\begin{array}{l}\text { GCGCTCATCA } \\
\text { GCGCTCATCA }\end{array}$ \\
\hline & & \\
\hline 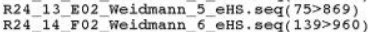 & & \\
\hline 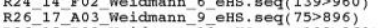 & & CAA \\
\hline Bo3_Weidmann-10 eHs. eqeq & & $\mathrm{CA}$ \\
\hline & & \\
\hline i-eHs: & $\rightarrow$ & GGACATTTCTAATGCTGTAGTTCCAAACTCAGCCCTCATTGCTC? \\
\hline $\ln ^{n-}$ & & \\
\hline & & \\
\hline & & \\
\hline & & \\
\hline
\end{tabular}




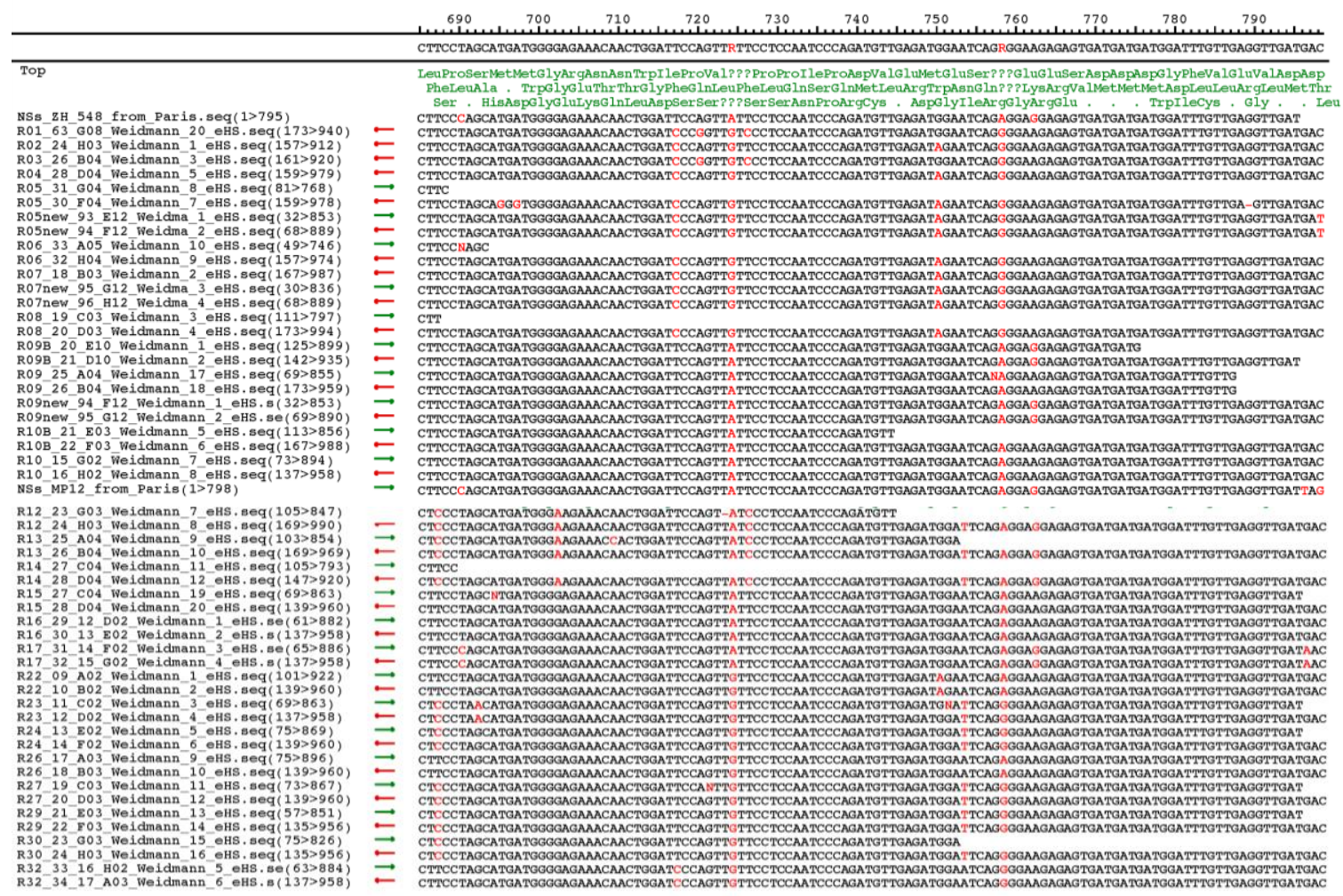

${ }^{800} .{ }^{810}{ }^{820}$ ACAAGGACGACGATGACAAGTGA

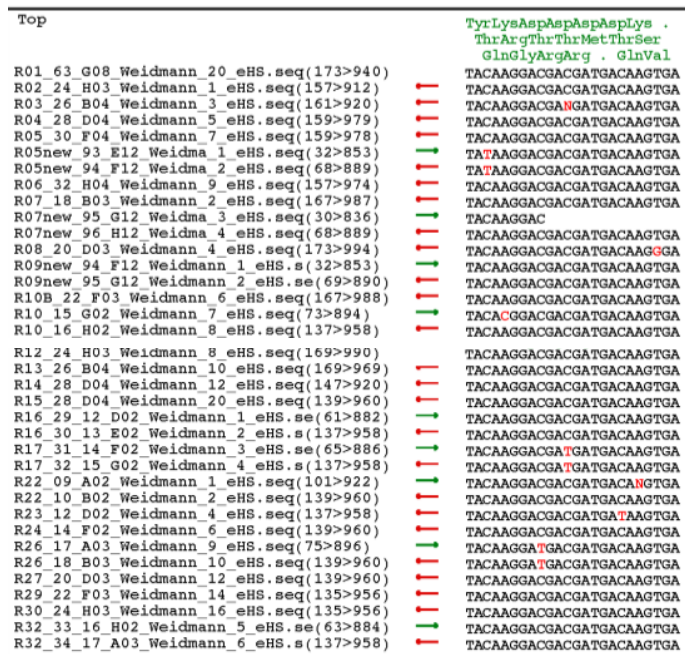




\section{Acknowledgements}

My profound gratitude goes to Dr. Martin Spiegel and Dr. Manfred Weidmann, Dr. Meik Dilcher and to Prof. Dr. med. Frank T. Hufert for their excellent care, intense discussions and enormous support to this work. I am particularly thankful for their really infernal patience and inestimable contribution to my training as scientists. Thanks to the staff of the virology institute in Göttingen.

I appreciate Prof. Dr. med. Gerhald Hunsmann and Prof. Dr. med. Uwe Groß kind acceptance to be respectively the referee and the co-referee of my thesis.

This work has been made possible by the financial support of the World Bank as part of the PSAOP/ISRA/Ministry of Agriculture. I would like to acknowledge Andrea Paluschkiwitz for excellent technical assistance and high professionalism and care, to Bastian Dörrbecker for his generous help. We express our gratitude to the General Director of the "Institut Senegalais de Recherche Agricole" Dr. Macoumba Diouf, the Scientific Director Dr. Alioune Fall, the training manager Dr. Aly Ndiaye and the Head of the "Laboratoire National d" Elevage et de Recherches Vétérinaires", Dr. Yaya Thiongane, allowing me to carry out this $\mathrm{PhD}$ thesis work.

My highest gratitude goes to my beloved family Diale Ndiaye, Fatou Tall, Papa Makhtar Lo, Yaya Ben Moustapha Lo, Ndeye Fatim Lo, Maodo Malick Lo and Rokhaya Malick Lo - my best team to go through life.

My gratitude goes to Serigne Moustapha Sy Djamil's family, my second family and all Sy's Family. Serigne Cheikh Saliou M’Backé and all Mbacke's Families.

Pious thought to my parents Makhtar Lo and Seynabou Sèye, my first wife Fatim Diagne, my brother Abdoulaye Lo, El Hadji Cheikh Ngoné Ndiaye, Adjaratou Fatou Diop, Ndiatou Fall, El Hadji Malick Ndiaye, Loly Diop etc.

Last but not least I always address many thanks to my brothers Djibril Lo and Babacar Lo Yaram Lo and their families, my mother's family Ahmed Seye's and Abdoul Aziz Seye's families, Khadidiatou Seye, Maodo Lo and family, Modou Guèye and Galaye Faye's Family, my father family El Hadji Moussa Lo's family, Yaram Lo's Family, Massar Lo's Family. My gratitude goes also to Adjaratou Ngoné Niass, Sakoki Ndiaye and her wife Ngane Ndiaye, Modou Ndiaye, Serigne Mbacké, Modou Astou Niasse, Talla, Seyni and all the family, Dr Fatou Diop, El Hadji Moussa Tall's family, Mere Issa Ndiaye and all the Ndiaye's family, Fadiop Ndiaye, Ndoumbé Diop, Maty Guissé. My cousins Abdoulaye Diagne, Bilal Diagne, El hadji Moustapha Diagne, Diarra Diagne and all the family. Malick Koné and his family, Astou Keita, Lala Keita and her husband Diallo, Oumar Sall, Ngagne Sène.

Many thanks to my colleagues at ISRA/LNERV: Dr Abdoulaye Niass, Mariame Diop, Mme Ndoye, Dr Papa Seck, Dr Momar Talla Seck, Dr Mbaye Mbengue, Dr Alpha Diallo, Dr EL H, Traore, Dr Alkaly, Yacine Samb, Moussa Thialaw, Moussa Diouf, Dane Sèye, Dr Saliou Ngom, Ndèye Salane Faye, Adam Mbaye, Mme Ndèye Fatou Tall, Youssouph Sané, Jocob, Cheikh Dia, Mme Basse, Oumar Bougaleb, Dr Omar Talla Diaw, Dr Assane Guèye Fall, Souleye Cissoko, Mme Gueye Couta Camara and Assy Gomis. 


\section{Curriculum Vitae}

- Name: Modou Moustapha Lô

- Date and place of birth: March $12^{\text {th }} 1958$, Gossas in Senegal

- Marital status: married

- Nationality: Senegalese

\section{School, university course and Post University}

2007-2010: University of Göttingen: Candidate for the degree of Doctor of Philosophy $(\mathrm{PhD})$ in the Department of Virology of the Medical Faculty 2003 - 2004: Institute Of Tropical Medicine in Antwerp in Belgium: Master of Sciences in Animal Health (Option disease control), obtained score B

July 1998 to november1998: Freie Universität of Berlin in Germany: Department of Tropical Veterinary Medicine and Epidemiology: Qualified Certificate in Seroepidemiological Diagnosis in the Laboratory and in Animal Disease Control.

1984 - 1987: Dakar University Cheikh Anta Diop in Senegal: Faculty of sciences and Technology: Bachelor in Natural Science

1981 - 1984: Inter-State School of Sciences and Veterinary Medicine de Dakar

1980 - 1981: Dakar University Cheikh Anta Diop in Dakar: Faculty of Science Technology and: CPEV (Preparatory Certificate in Veterinary study)

1977 - 1979: Charles De Gaulle's College at ST Louis (Senegal): diploma of High Degree option D (Mathematical Sciences and Natural Sciences)

1973 - 1976: Gossas secondary College: diploma BEPC

1967 - 1972: Gossas primary school: diploma CEPE and end of the primary school.

\section{Professional experiences}

From $26^{\text {th }}$ May to $29^{\text {th }}$ May 2009. Complete training course in Biosafety level 3 (BSL3) at the Institute of Virology Faculty of Medicine Georg-August University of Göttingen, Germany

From $20^{\text {th }}$ November to $1^{\text {st }}$ December 2006 participated in and completed the Interregional Training Course On Rapid Diagnosis of Avian Influenza (Bird Flu) held at the IAEA Laboratories, Seibersdorf, Austria.

From July $\mathbf{0 7}^{\text {th }}$ to $\mathbf{1 1}^{\text {th }}$ 2006: Successfully completed training course in "Biosafety and Biosecurity in Animal Research and Veterinary Clinics at Colorado State University, Fort Collins, Colorado, USA

From February $27^{\text {th }}$ to March $\mathbf{0 3}^{\text {rd }}$ 2006: training course in: "High Pathogenic Avian Influenza (HPAI) Diagnosis" At AMES, IOWA, USA. 
From June $14^{\text {th }}$ at July $28^{\text {th }}, \mathbf{2 0 0 5}$ at Alken in Belgium: Training course in sustainable agriculture "integration agriculture-breeding, forestation, production of fodder, artificial insemination in the pig, milk production and transformation in cheese".

From September $15^{\text {th }}, 2003$ to July $15^{\text {th }}, 2004$ at the Institute of Tropical Medicine in Antwerp in Belgium: Training course for Master of Science in Health Animal (Option Disease Control).

From the June $1^{\text {st }}$ to $14^{\text {th }}, 2004$ : Development of serologic technique to diagnose RVF at Unit of Emerging Viruses at the University of Méditerannée in Marseille, France.

From February $19^{\text {th }}$ to March $1^{\text {st }} \mathbf{2 0 0 2}$ in the International Center of Trypanotolerance, Banjul, Gambia: participation in the regional training course on "Serological and PCR techniques to diagnose zoonotic diseases in ruminants"

From July $0^{\text {th }}$ to $^{\text {th }}$ November1998: Freie Universität Berlin in Germany: Department of Tropical Veterinary Medicine and Epidemiology: Qualified Certificate in Seroepidemiological Diagnosis in the Laboratory and in Animal Disease Control.

From October $21^{\text {th }}$ to November $\mathbf{0 8}^{\text {th }} 1996$ participation in the international training course organized by the EISMV and GTZ/Laboratory service on the "Serological methods or the diagnosis of zoonotic diseases of ruminants".

From December $06^{\text {th }}$ to $31^{\text {st }} 1993$ training course at the laboratory of the Arboviruses on "serological and virological diagnosis and antigen preparation of Rift Valley Fever" at Pasteur Institute of Dakar.

From December 1989 Researcher Assistant in Animal Health Program, (LNERV)/ISRA

From July 1989 to November 1989 training course at the Institute of Food Technology: laboratory of microbiology: quality control of the animal products and water.

From 1988 to 1989 Head of the Chamel Dairy milk production Laboratory: "Tiviscky" in Mauritania

From 1987 to 1989. Assistant in the National Research Center in Veterinary in Nouakchott at Islamic Republic of Mauritania.

\section{Research activities in ISRA/LNERV, Senegal}

1. Surveillance of Rift Valley Fever in Senegal and all transboundary diseases (RVF, FMD, AI)

2. Trial vaccine with a reassortant $566 \mathrm{RVF}$ virus (reassortant) vaccine against the rift valley fever), VLP RVFV vaccine on sheep.

3. Field investigation on diseases outbreaks

4. Development and improvement of vaccine quality

- Attenuation of the Ehrlichia ruminantium strain isolated from Niayes Dakar, Senegal in susceptibles primairy endothelial cells.

- Participation in production and quality control on thermostable batch of seed vaccine $I_{2}$ against New Castle Disease in accordance to the standards of International Office Of Epizootie. 
5. Quality control of viral vaccines: Vaccines against Sheep and Goat Pox, African Horse Sickness, peste des Petits Ruminants, New Castle, Avian Pox ect.

\section{Activities as trainer}

1. From $12^{\text {th }}$ to $17^{\text {th }}$ July 2010 Trainer for the RESOLAB(ECTAD in Bamako) during the training course on necropsy, sampling, biosecurity and biosafety in the veterinary laboratory diagnostic in ISRA/LNERV in Dakar, Senegal.

2. From $22^{\text {nd }}$ January to $2^{\text {nd }}$ February 2007: participation as trainer of the regional course on avian influenza diagnostics held in Dakar, Senegal

3. Trainer for African Unit UA/IBAR staff in charge of medical and veterinary laboratories and involved in surveillance of AI from Senegal, Cote d'Ivoire, Mali, Togo, Burkina Faso, Guinea, Guinea-Bissau, Mauritania, Tunisia, Algeria, Cape Verde, Niger, Morocco in the field of AI diagnostic and biosafety and biosecurity organized in Institute Pasteur/ISRA Dakar, Senegal $\left(30^{\text {th }}\right.$ October to $10^{\text {th }}$ November 2006)

4. From July 17th to 20th 2006 trainer for the CONAGA (Comite National de Lutte contre la grippe aviaire au Senegal) to persons future trainers on the diagnosis methods of highly pathogenic avian influenza, and the concepts of biosecurity and biosafety in Saly; Senegal

5. Participation in the training of African veterinary laboratory staff on the diagnosis of highly pathogenic avian influenza with serological, virological and molecular diagnostics, Dakar from June $26^{\text {th }}$ to 30rd 2006. Organized by FAO in collaboration with the reference laboratory of FAO in Padova in Italy.

6. Expert of APHIS-USDA to implement serological diagnosis methods of avian influenza at the national veterinary laboratory in Garoua, Cameroun April 2006

\section{Scientific papers}

1. Bessin R, Zeller H, Thiongane Y, Lo M M, Belem, A M G., Rôle du virus de la fièvre de la vallée du Rift dans les avortements des petits ruminants au Burkina faso, Bull. Anim.Hlth.Prod.Afri.(1998), 46, 181-186.

2. Thiongane Y, Zeller H, Lo M M, Fati Nd A, Akakpo, Baisse de l'immunite naturelle vis a vis de la fievre de la vallee du Rift chez les ruminants domestiques du bassin versant du fleuve Senegal apres l'epizootie de 1987, Bull Path Exot, 1994, 86 : 1-2.

3. Diaite A, Gueye A, Thiongane Y, Lo M M, Dieye T Nd \& Vassiliades G., Observation dans les Niayes du Senegal d'une souche de Trypanosoma (Dutttonella) vivax transmissible d'un bovin a des souris par la seringue., Rev. Elev. Med. Vet. Pays Trop., 1998, 51 (2): 127-129.

4. Thonnon J, Michel P, Thiongane Y, Lo MM, Sylla R, Vercruysse J, Rift valley fever surveillance in lower Senegal basin: update 10 years after epidemic, Trop. Med. And Int. Health, Aug. 1999, 4 (8): 580-485. 
5. Thiongane Y, Bada-Alambedji R, Morou A, Lo M M, Mathiot C, Gonzalez J P, Akakpo A J, La fièvre de la vallée du Rift: Enquete serologique chez les ruminants domestiques dans la region du fleuve au Niger, Revue Africaine de sante et de Productions Animales, 2004, 2 (1): 37-41.

6. Weidmann M, Sanchez-Seco MP, Sall AA, Ly PO, Thiongane Y, Lo MM, Schley H, Hufert FT. Rapid detection of important human pathogenic Phleboviruses. J Clin Virol. 2008 Feb; 41(2):138-42.

7. Ergünay K, Saygan MB, Aydogan S, Lo MM, Weidmann M, Dilcher M, Sener B, Gascelik G, Pinar A, Us D, Sandfly Fever Virus Activity in Central/Northern Anatolia, Turkey: First Report of Toscana Virus Clin Microbiol Infect, 2010 [epub ahead of print]

8. Lo MM, Spiegel M, Hunsmann G, Weidmann M, Hufert FT, Thiongane Y, Sall A.A. Variability of the IFN- $\beta$ promoter repressing activity of NSs proteins derived from field isolates of Rift Valley Fever virus. In N.E. Odongo, M.Garcia \& G.J. Viljoen (eds), Sustainable Improvement of Animal Production and Health. Food and Agriculture Organization of the United Nations, Rome, Italy (In press).

\section{Oral presentations at international meetings}

1. Situation of Rift Valley Fever sero-survey in the left bank of Senegal River Basin during these two last years 2005 and 2006 oral presentation during the $2^{\text {nd }}$ Research Coordinated Meeting from $5^{\text {th }}$ to $9^{\text {th }}$ March 2007 with IAEA at Nairobi, Kenya.

2. "Variability of the IFN- $\beta$ repressing activity of NSs proteins from 26 wt RVFV isolates and expression of their NSs in Vero E6 cell" oral presentation at the FAO/IAEA International Symposium on "Sustainable Improvement of Animal Production and Health" to be held in Vienna, Austria from 8 to 11 June 2009. This paper has been assigned the number IAEA-CN-174-244.

3. Variability of the IFN- $\beta$ repressing activity of NSs and their immunomodulatory properties, oral communication at Fourth International RIPROSAT Symposium "Transboundary Diseases and Food Security" September 1st, 2nd and $3^{\text {rd }}$ Quito, Ecuador.

\section{Other activities}

1. Member of the National committee of Prevention of Avian Influenza in Senegal (CONAGA) since 2005.

2. Member of the International Network of Tropical Animal Health Graduates Belgium. 